\title{
CHARACTERIZATION OF BERRY AND WINE AROMA DEVELOPMENT IN INTERSPECIFIC HYBRIDS IMPORTANT TO MISSOURI USING A METABOLOMICS BASED APPROACH
}

\author{
A Dissertation presented to \\ the Faculty of the Graduate School \\ at the University of Missouri-Columbia \\ In Partial Fulfillment \\ of the Requirements for the Degree \\ Doctor of Philosophy \\ by \\ MANI AWALE
}

Dr. Misha Kwasniewski, Dissertation Supervisor

JULY 2020 
The undersigned, appointed by the dean of the Graduate School, have examined the dissertation entitled

\section{CHARACTERIZATION OF BERRY AND WINE AROMA DEVELOPMENT IN INTERSPECIFIC HYBRIDS IMPORTANT TO MISSOURI USING A METABOLOMICS BASED APPROACH}

presented by Mani Awale, a candidate for the degree of doctor of philosophy, and hereby certify that, in their opinion, it is worthy of acceptance.

Professor Misha Kwasniewski

Professor Walter Gassmann

Professor Laszlo Kovacs

Professor Ruthie Angelovici 
Dedicated to my late father who has always encouraged me to pursue my dreams, to my mom who has helped me navigate my path to my dreams and to my late grandmother who had always been my pillar of strength. Thanks, Mom. 


\section{ACKNOWLEDGMENTS}

I am extremely grateful to the number of faculties, friends, and my family for all the encouragement, guidance, love, and support given to me throughout this adventurous, joyful as well as challenging journey.

First, I express my profound gratitude to my advisor Dr. Misha Kwasniewski for providing me the precious opportunity to work on several interesting projects at the University of Missouri-Columbia. I am indebted to him for all his guidance, love, care, and immense knowledge throughout my research period. I still remember it was a challenging transition for me to join the lab, but I was excited. Before joining the lab, my background was in Plant (Grapes) Genetics. During my MS, I got a few opportunities to visit the local vineyards and winery and learned about the winemaking process, which was a fascinating moment. This reminded me of how my mom and grandma used to make Nepalese traditional wine and liquor back at home and got me more excited. This intensified my interest in the field of viticulture and enology after realizing that knowledge of genetics along with various management practices and winemaking processes can be an asset to improve the wine industry. I did not know how challenging the analytical chemistry will be for me until I started doing it myself. Thank you, Misha, for providing me an unquantifiable amount of guidance and support especially on chromatography and analytical chemistry without which I would not be able to succeed.

I also thank Drs. Walter Gassmann, Ruthie Angelovici, and Lazlo Kovacs for serving in my committee and for providing me with constructive and meaningful comments and suggestions. Thank you very much for writing several recommendation letters on my behalf to different scholarship committees and organizations. I am truly indebted for that. My deepest gratitude goes to the Department of Plant Science, faculty 
and staff for all their help and support. I am very much thankful to Grape and Wine Institute and NSF VitisRoots for funding my research. Thank you to the entire VitisRoots team especially to Dr. Allison Miller and Dr. Anne Fennell (my former MS advisor, SDSU) for being my role model for Women in Science and all the help and support.

My sincere thanks to Connie Liu for her contribution and assistance in running my samples and for her love and care in the lab. Thank you, Connie, for being both my mentor as well as my friend. Your contribution to running samples and troubleshooting has helped me to move forward. I cannot forget my mentor and friend Megan for her useful suggestions, motivation, and hearing to me when I needed someone. Thank you for providing me the positive vibes and the right attitudes all the time. I am thankful to all my friends and lab colleagues for their love and cooperation. Shout out to Alex, Alan, Joe, Atzin, and lovely Maggie for all the help and fun.

I appreciate the love and support given to me by my family, my mom, without which I couldn't stand at this stage. She has been an example of courage and strength to me. Although she can barely read and write, her love, sacrifice and continued support for my education even after the passing of my Dad at an early age, has motived me to pursue this dream, and to be determined to make this dream come true. My grandma and my aunt also have had my back throughout my life. I thank them and my brother, Abin, and my sister in law, Mimi for always being with me. My interest in the field of agriculture developed from our family farms where we used to plant rice in summer. Thank you for teaching me to plant rice and instilling me that hard work and patience is required to bear fruit.

I also owe this to my childhood best friends Sabina and Sareena who always cheered me throughout my difficult times. I also thank my in-laws especially Vivek's 
sisters who had been the sisters I always wanted and who had been so welcoming and supportive to me throughout this time. Lastly, my dearest love and appreciation goes to my husband, Vivek, for the motivation, encouragement, love, and support and for always being with me in my sorrow and happiness. Thank you for listening to those countless problems and always motivating and supporting me when I was ready to give up. Thank you for believing in me when I doubted myself. To anybody that I failed to mention, I thank you all so much for your help. 


\section{TABLE OF CONTENTS}

ACKNOWLEDGMENTS _................................................................................. ii

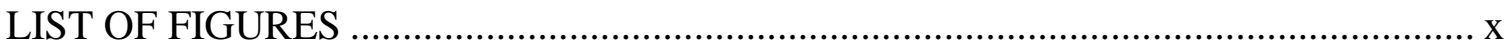

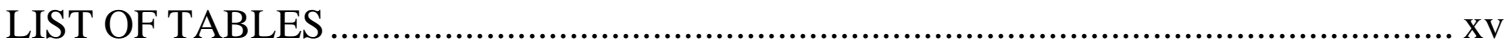

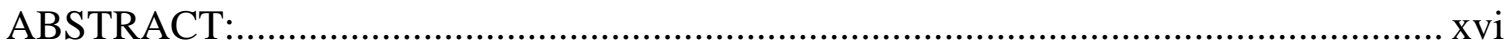

Chapter 1: General Introduction and literature review ………………………………... 1

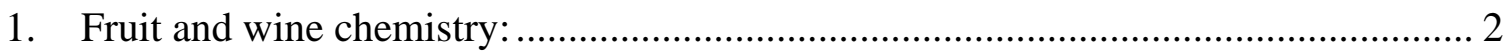

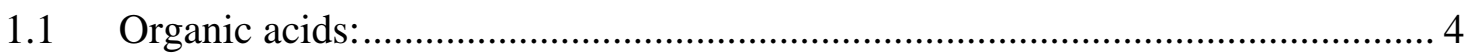

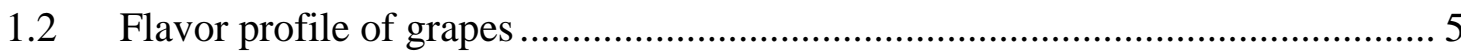

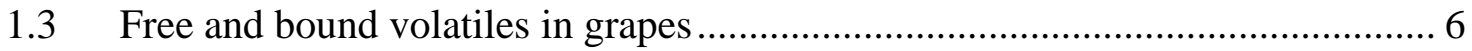

1.4 Grape aroma molecules:...................................................................... 8

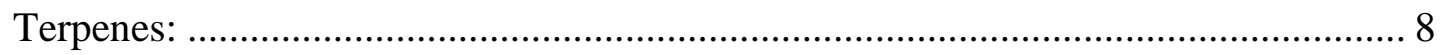

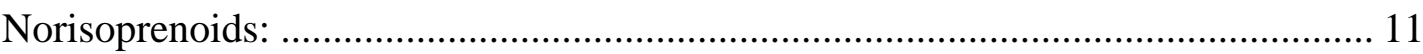

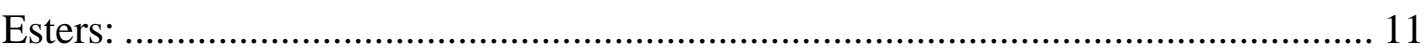

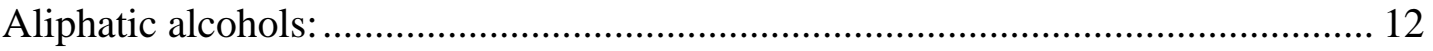

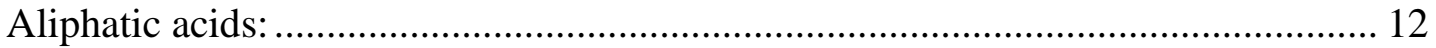

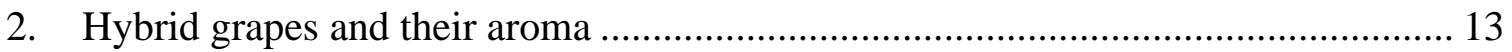

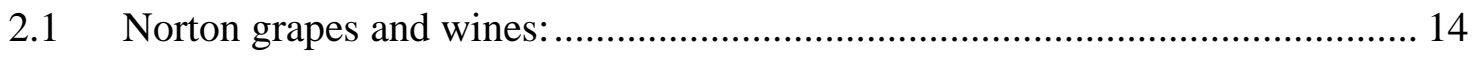

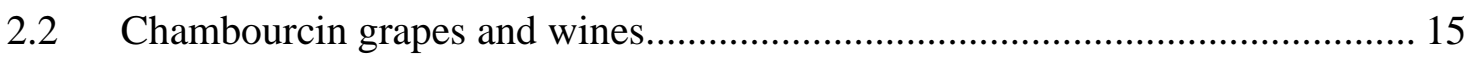

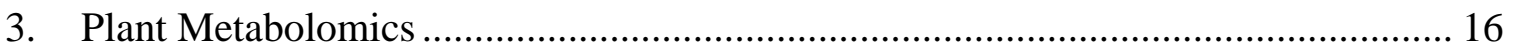

3.1 Targeted and untargeted metabolomics............................................................ 17

3.2 Untargeted Metabolomics Workflow............................................................ 18

3.3 Solid-phase microextraction (HS-SPME): ........................................................ 19

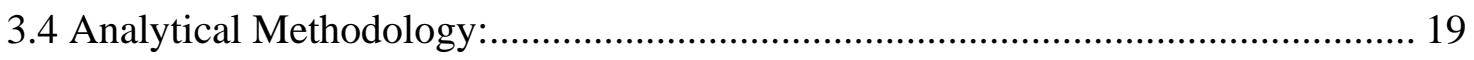

3.5 Data pre-processing and pre-treatment: ............................................................ 21

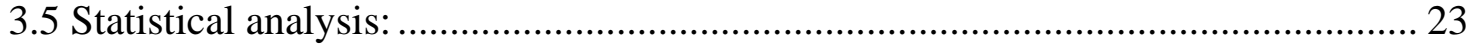

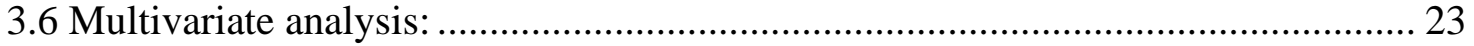

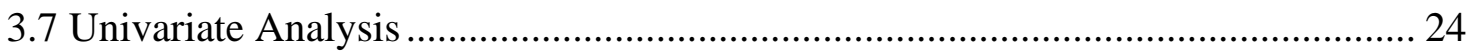

4 Development and use of rootstocks in viticulture: ................................................. 26

5 Rootstocks effect on grapes and wine chemistry: ................................................... 27 


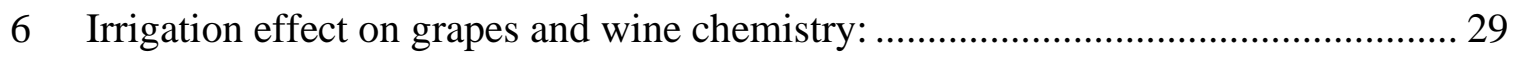

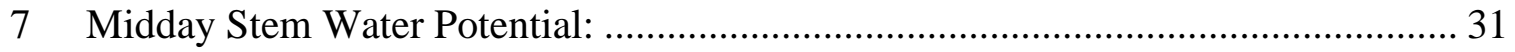

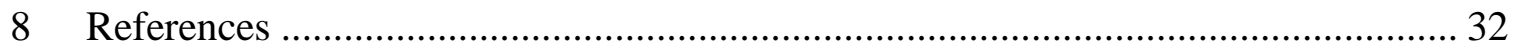

Chapter 2: Identification of aroma markers for Norton and Cabernet Sauvignon berries

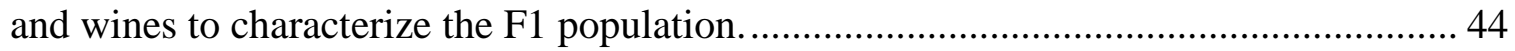

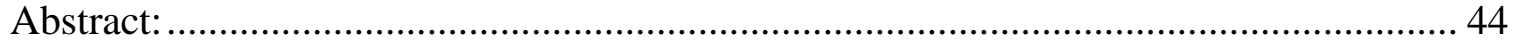

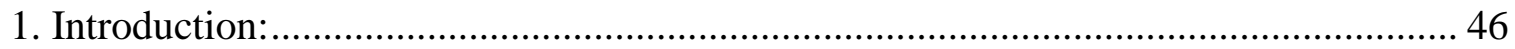

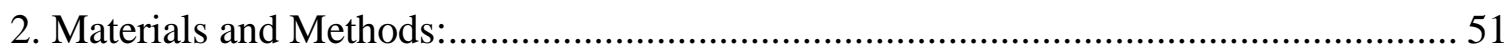

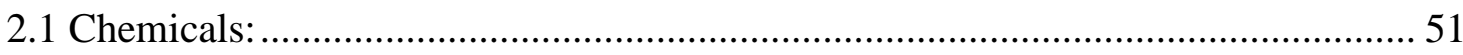

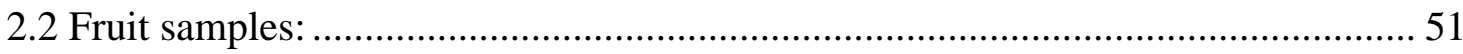

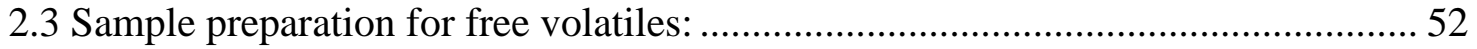

2.4 Sample preparation for total/bound volatiles: .................................................... 53

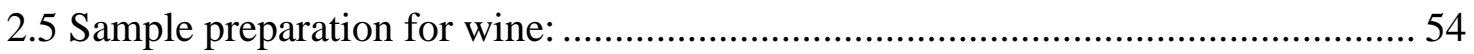

2.6 Headspace solid-phase microextraction gas chromatography mass spectrometry

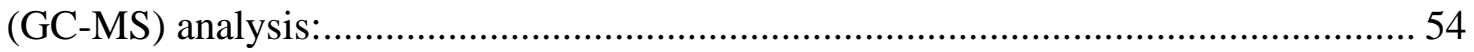

2.7 Data processing using untargeted metabolomics analysis: ............................... 55

2.8 Identification of compound and confirmation:................................................ 56

2.9 Screening F1 population using identified volatiles: ......................................... 57

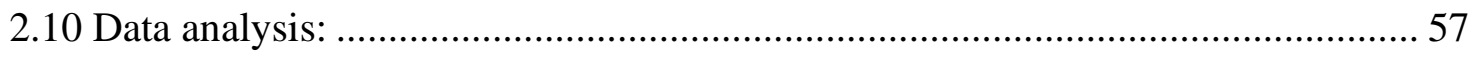

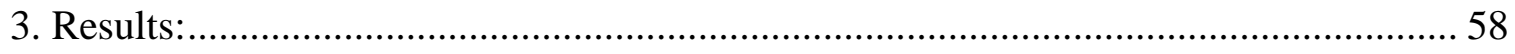

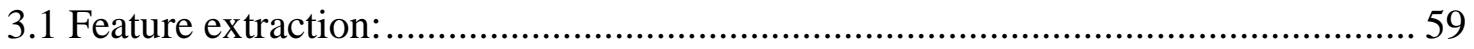

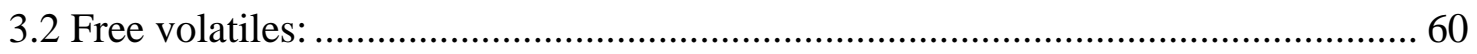

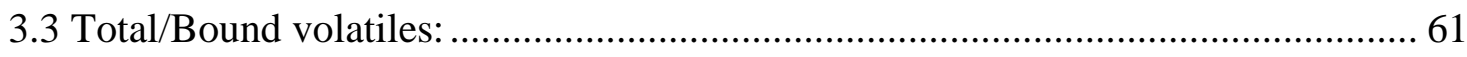

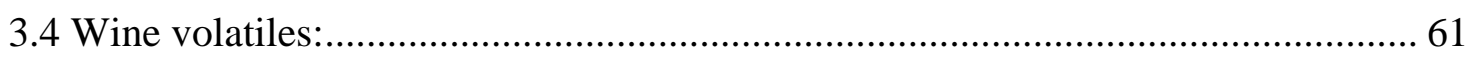

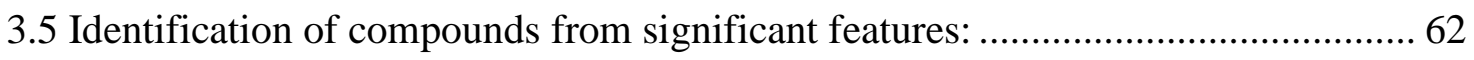

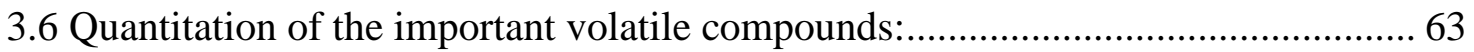

3.7 Characterizing F1 population of Norton and Cabernet Sauvignon with the identified

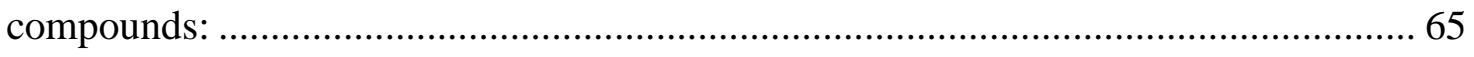

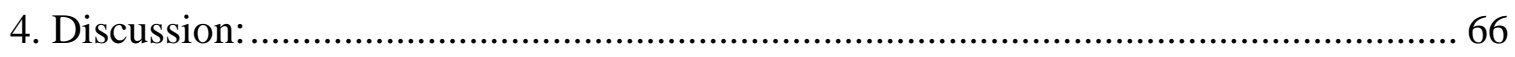

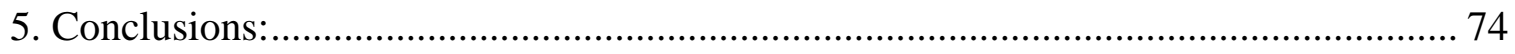

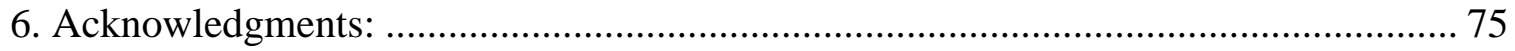




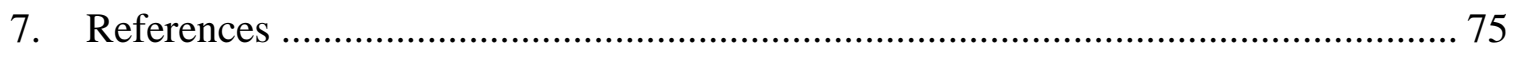

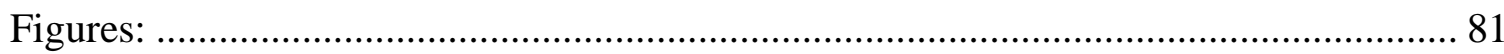

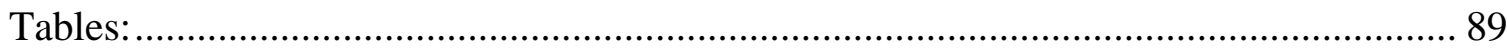

Chapter 3: Rootstock Modulation of Wine Aroma Compounds Under Varied Irrigation

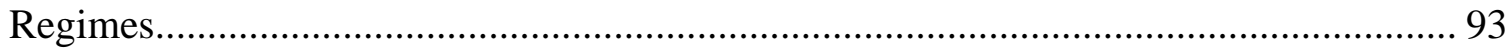

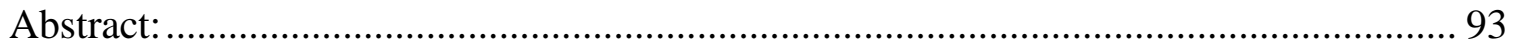

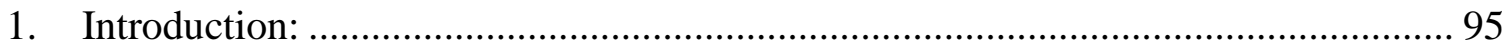

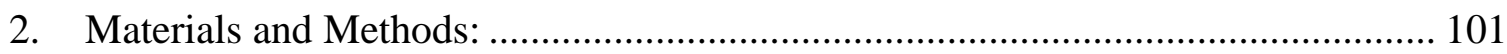

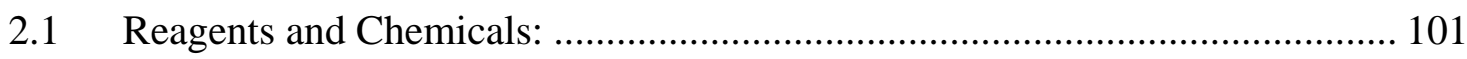

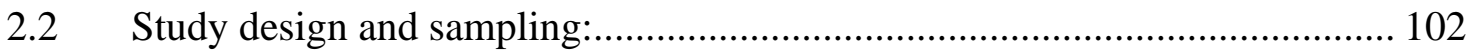

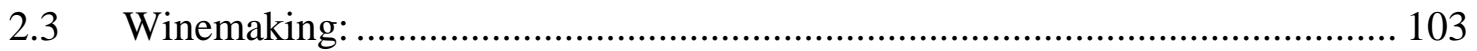

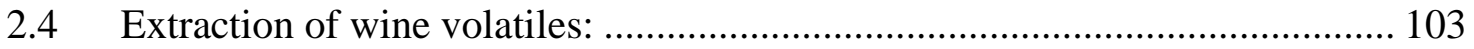

2.5 HS-SPME-GC-MS and HS-SPME-GCMS/MS:_........................................ 103

2.6 Data processing using untargeted metabolomics analysis: ............................... 104

2.7 Identification and confirmation of the compounds: ....................................... 104

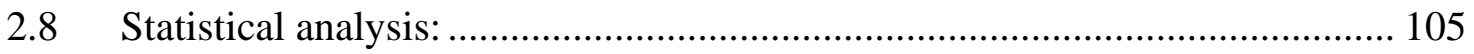

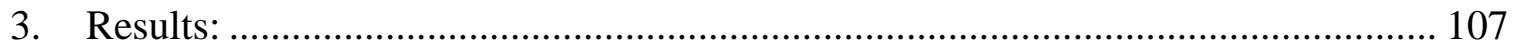

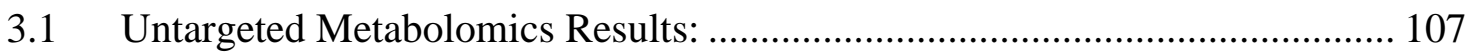

3.2 Multivariate analysis of wine features: ......................................................... 109

3.3 Compound Identification and confirmation: ................................................. 111

3.4 Quantitation of the compounds: .................................................................... 112

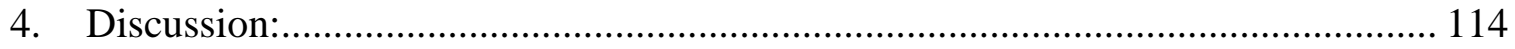

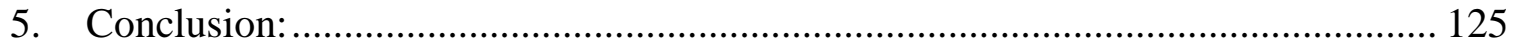

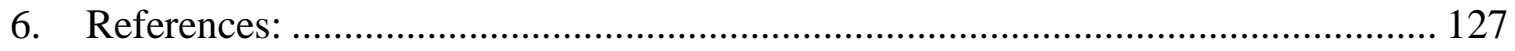

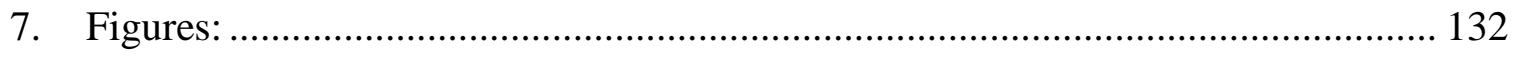

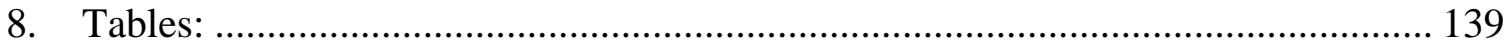

Chapter 4: Rootstocks and Irrigation impact free and total volatiles in Chambourcin berries using a metabolomics-based approach................................................................ 143

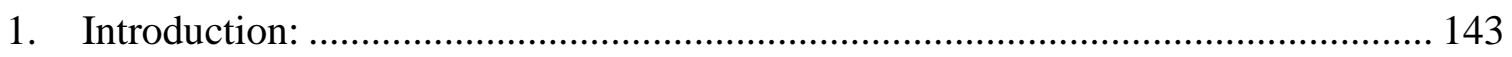

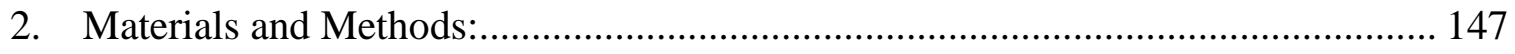

2.1 Experimental design and sampling and sample processing: ........................... 147 
2.2 Extraction of free and total volatiles from berries:

2.3 Head Space Solid Phase Microextraction Gas Chromatography-Mass

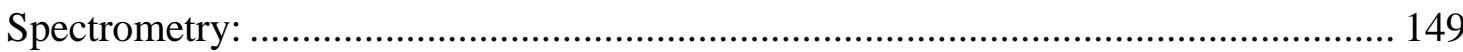

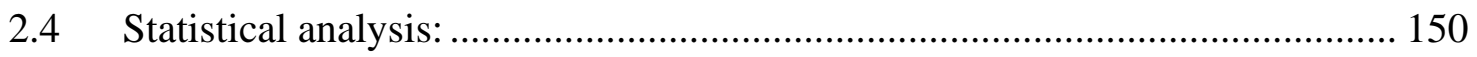

2.5 Identification and confirmation of the compounds: ................................. 151

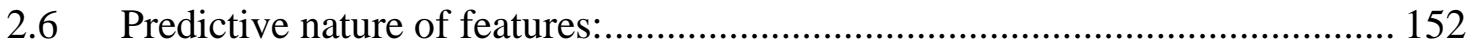

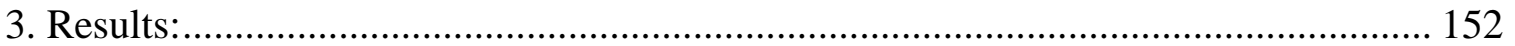

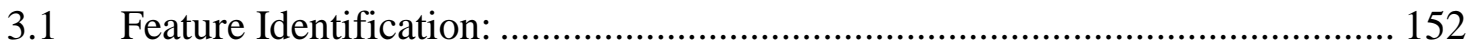

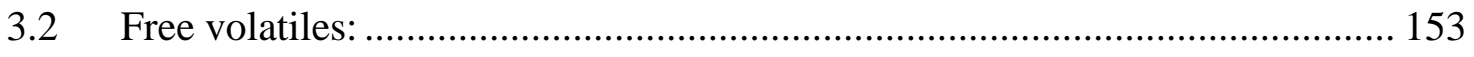

3.2.1 Impact of phenology and year on free volatiles:................................. 155

3.2.2 Impact of rootstock on free volatiles: .............................................. 156

3.2.3 Impact of irrigation on free volatiles: .................................................. 157

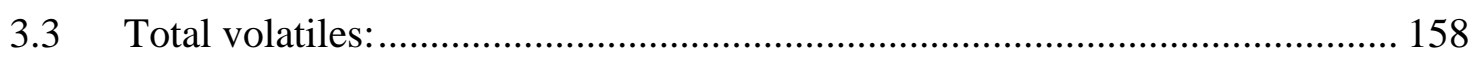

3.3.1 Impact of phenology and year on total volatiles:................................. 159

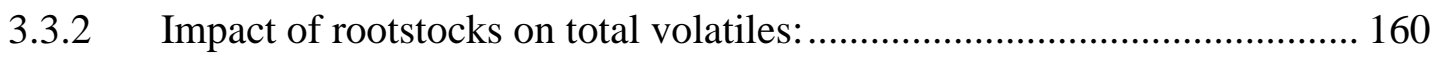

3.3.3 Impact of irrigation on total volatiles: ............................................... 161

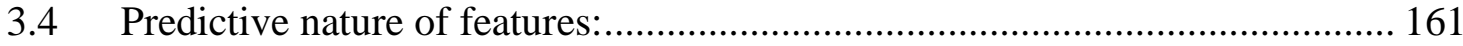

3.5 $\beta$-Damascenone concentration differences due to rootstocks and irrigation: .. 162

3.6 Total Methyl Salicylate is impacted by rootstocks: ..................................... 164

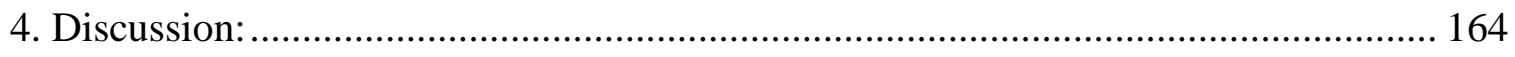

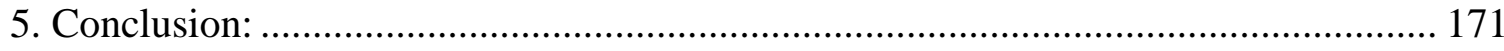

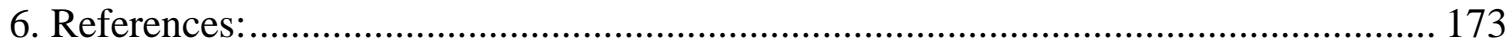

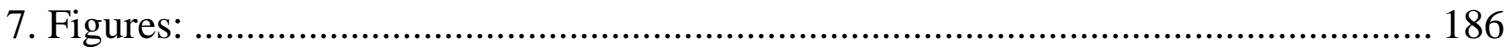

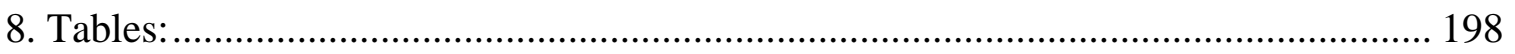

Chapter 5: Rootstock and irrigation impact berry chemistry and organic acids............ 203

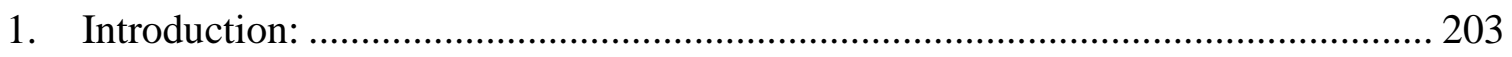

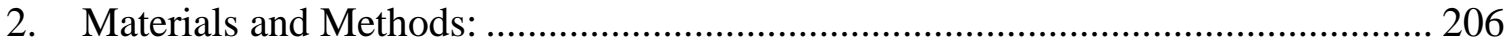

2.1 Experimental design and sampling and sample processing: ............................. 206

2.2 Midday Stem Water Potential: ................................................................... 206

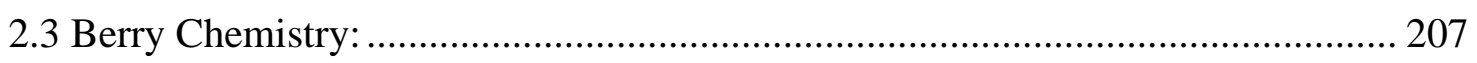


2.4 Analysis of Organic acid concentrations in Berries using Reverse-Phase UltraHigh-Performance Liquid Chromatography: ............................................................ 207

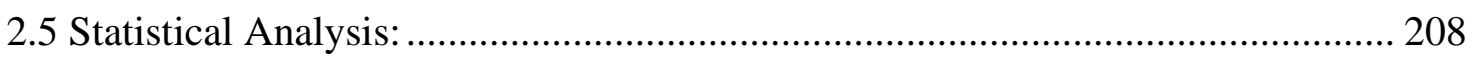

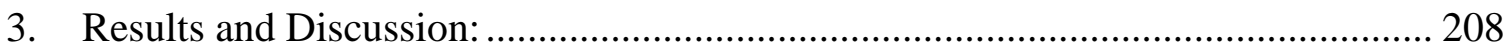

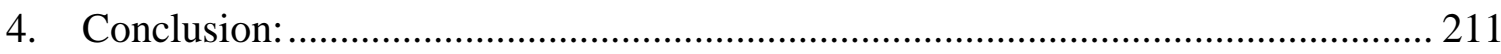

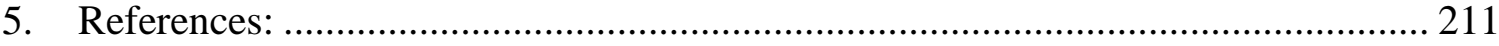

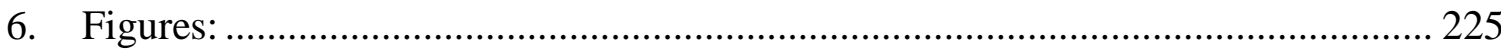

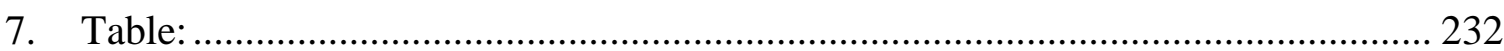

Chapter 6: Conclusions and future works:.............................................................. 234

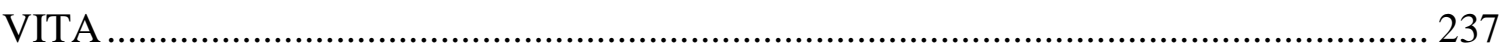




\section{LIST OF FIGURES}

Figure 2.1: Heatmap of the top 25 most influential features for differentiating free volatiles between Norton and Cabernet Sauvignon berries. While only top contributors are shown the heatmaps were generated using all the features. The rows in the heatmap represent features $(\mathrm{M}(\mathrm{m} / \mathrm{z})$.T(time in minutes) and the columns indicate sample categories. The colors of the heatmap cells indicate the abundance of compounds across different samples. The color gradient, ranging from dark blue through white to dark red, represents low, middle and high abundance of a compound.

Figure 2.2: Principal components analysis (PCA) scores plot for the distribution of (A) free volatiles, (B) total volatiles and (C) wine volatiles features in Norton and Cabernet Sauvignon $(\mathrm{Cab})$ grapes and wines. The ellipses show $95 \%$ confidence interval. There is a separation based on cultivar for free, total and wine metabolic features.

Figure 2.3: Heatmaps of the abundance of the volatiles in Norton and Cabernet Sauvignon (Cab) (A) free volatiles, (B) Total volatiles and (C) Wine volatiles. Heatmaps were created using average quantitative values based on 2-Octanol IS. The rows in the heatmap represent volatile compounds and the columns indicate cultivars. The colors of the heatmap cells indicate the abundance of compounds across different samples. The color gradient, ranging from dark blue through white to dark red, represents low, middle and high abundance of a compound

Figure 2.4: PCA scores map of the model obtained for (A and B) free, (C and D) total and (E and F) wine volatiles indicating the distinct separation of Norton and Cabernet Sauvignon for wine volatiles using quantitative data and the biplot showing volatiles contributing to the differences in Norton and Cab wine volatiles.

Figure 2.5: Boxplots showing the distribution of Methyl Salicylate in Norton and Cabernet Sauvignon (Cab) (A) free volatiles, (B) Total volatiles and (C) Wine volatiles. The $y$-axis denotes concentration in $u g / L$ obtained semi-quantitatively relative to 2 Octanol.

Figure 2.6: Boxplot showing the distribution of $\beta$-Damascenone in Norton and Cabernet Sauvignon (Cab) (A) Free, (B), total and (C) Wine volatiles. The y-axis denotes concentration in $\mathrm{ug} / \mathrm{L}$.

Figure 2.7: Boxplot showing the distribution of TDN in Norton and Cabernet Sauvignon (Cab) (A) Free, (B), total and (C) Wine volatiles. The y-axis denotes concentration in $\mathrm{ug} / \mathrm{L}$

Figure 2.8: Boxplots demonstrating the variability of the (A) free and (B) total volatiles in F1 population cross from Norton and Cabernet Sauvignon (Cab). The concentrations of the volatiles in ug/L were log-transformed. 
Figure 2.9: Frequency distribution of (A) Free Eugenol, (B) Total Eugenol, (C) Free methyl Salicylate and (D) Total methyl salicylate in F1 population of Norton and Cabernet Sauvignon. The concentration values in $\mathrm{ug} / \mathrm{L}$ were log-transformed.

Figure 3.1: Flowchart of the Subtle Differentiation Metabolomics workflow to identify the minute differences in wine aroma due to rootstocks and irrigation. 132

Figure 3.2: Heatmaps of the top 25 most influential features for differentiating wine volatiles by (A) irrigation regime in 2017, (B) irrigation regime in 2018, (C) rootstocks in 2017 and (D) rootstocks in 2018. While only top contributors are shown the heatmaps were generated using all the features. The rows in the heatmap represent features $(\mathrm{M} / \mathrm{m} / \mathrm{z})$ $\mathrm{T}$ (time in minutes) and the columns indicate sample categories. The colors of the heatmap cells indicate the abundance of compounds across different samples. The color gradient, ranging from dark blue through white to dark red, represents low, middle and high abundance of a compound.

Figure 3.3: A) Venn diagram showing common and unique features between wines in 2017 and 2018. Only 45 features were common in both years showing yearly differences in metabolites. B) Venn diagram showing common and unique features between rootstocks and irrigation treatments in wines in 2017. 170 features were similar between rootstocks and irrigation treatments. C) Venn diagram showing common and unique features between rootstock and irrigation treatments in wines in 2018. Few features were similar between rootstocks and irrigation treatments.

Figure 3.4: PCA Scores plot of wine metabolite features separated by rootstocks in (A) 2017 and (B) in 2018 regardless of the irrigation treatment. PCA Scores plot of wine metabolite features separated by irrigation treatments in (C) 2017 and (D) 2018 regardless of the rootstock treatment. PCA was performed using log-transformed and autoscaled significant features.

Figure 3.5: A linear model was estimated for wine 2017 and 2018 PCs 1 to 20. The model included rootstock (own-rooted, '1103P', '3309C', 'SO4'), irrigation (Full, RDI, None), and rootstock by irrigation interaction. The 20 PCs capture $85.79 \%$ and $78.34 \%$ of the variance in 2017 and 2018 respectively. Only factors which explained a significant portion of the variance $(\mathrm{p}<0.05)$ are plotted. The percent variation explained by each factor is indicated using color gradient. 136

Figure 3.6: Boxplots of (A) $\beta$-Linalool (Linalool) and (B) Ethyl hexanoate concentrations differences in rootstocks and own-rooted Chambourcin. Rootstocks increased concentrations of Linalool and Ethyl nonanoate in wines 2018. Concentrations were measured in $\mathrm{ppb}(\mathrm{ug} / \mathrm{L})$.

Figure 3.7: A) Boxplots of $\beta$-Damascenone concentrations differences in rootstocks and own-rooted Chambourcin in 2017 and B) in 2018. C)Boxplots of 1,1,6-trimethyl-1,2dihydronapthalene (TDN) concentrations differences in rootstocks and own-rooted 
Chambourcin in 2017 B) in 2018. Both $\beta$-Damascenone TDN concentrations were found in higher in own-rooted Chambourcin in wines in both 2017 and 2018. Concentrations

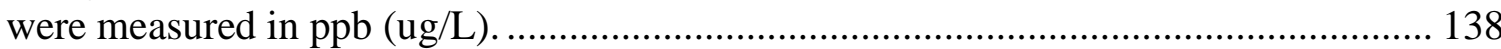

Figure 4.1: Heatmaps of the top 25 most influential features for differentiating (A) free volatiles by rootstocks, (B) free volatiles by irrigation regime, $(\mathrm{C})$ total volatiles by rootstocks and (D) total volatiles by irrigation regimes. While only top contributors are shown the heatmaps were generated using all the features. The rows in the heatmap represent features $(\mathrm{M}(\mathrm{m} / \mathrm{z})$.T(time in minutes) and the columns indicate sample categories. The colors of the heatmap cells indicate the abundance of compounds across different samples. The color gradient, ranging from dark blue through white to dark red, represents low, middle and high abundance of a compound. 186

Figure 4.2: PCA Scores plot of the model obtained for free volatiles due to (A) irrigation regimes $(\mathrm{B})$ rootstock and total volatiles due to $(\mathrm{C})$ and rootstock $\mathrm{D})$ detected by GC-MS. Samples collected from vines grafted with three different rootstocks and own-rooted Chambourcin subjected to three different levels of irrigation separated according to phenology and year.

Figure 4.3:A linear model was estimated for free volatiles PCs 1 to 20. The model included irrigation (Full, RDI, None), rootstocks (1103P, 3309C, Own and SO4), phenology (veraison, harvest), block (based on the position in the vineyard), and year (2017 and 2018). The amount of variance explained by each PC is listed in the table S1 in parenthesis with the first 20 PCs capturing a total of $85.36 \%$ of the variation in free volatiles. Only the factors which explained a significant portion of variance $(p<0.05)$ are plotted. The percent variance explained by each factor is indicated using color in the plot and also shown in table $\mathrm{S} 1$ 188

Figure 4.4 A linear model was fitted for (A) PC1 and (C) PC2 to extract features that are significant. Boxplot of a metabolite features differences between phenology in (B) PC1 and (D) PC2. The model included irrigation (Full, RDI, None), rootstocks (1103P, 3309C, Own and SO4), phenology (veraison, harvest), block (based on the the position in the vineyard), and year (2017 and 2018. Only the factors which explained a significant portion of variance $(p<0.05)$ are plotted. The percent variance explained by each factor is indicated using color in the plot.

Figure 4.5 A linear model was fitted for (A) PC8, (C) PC17 and (E) PC13 to extract features that are significant. Boxplot of (B)PC8, (D) PC17 and (F)PC13 differences between different rootstocks for free volatiles. The model included irrigation (Full, RDI, None), rootstocks (1103P, 3309C, Own and SO4), phenology (veraison, harvest), block (based on the position in the vineyard), and year (2017 and 2018. Only the factors which explained a significant portion of variance $(\mathrm{p}<0.05)$ are plotted. The percent variance explained by each factor is indicated using color in the plot. 
Figure 4.6: A linear model was fitted for (A) PC11, (B) PC20 and (C) PC14 respectively to extract features that are significant. D)Boxplot of one of the features indicating differences due to irrigation. The model included irrigation (Full, RDI, None), rootstocks (1103P, 3309C, Own and SO4), phenology (veraison, harvest), block (based on the position in the vineyard), and year (2017 and 2018. Only the factors which explained a significant portion of variance $(\mathrm{p}<0.05)$ are plotted. The percent variance explained by each factor is indicated using color in the plot.

Figure 4.7: A linear model was estimated for total volatiles. The model included irrigation (Full, RDI, None), rootstocks (1103P, 3309C, Own and SO4), phenology (veraison, harvest), block (based on the position in the vineyard), and year (2017 and 2018). The amount of variance explained by each $\mathrm{PC}$ is listed in the table $\mathrm{S} 1$ in parenthesis with the first 20 PCs capturing a total of $85.68 \%$ of the variation in total volatiles. Only the factors which explained a significant portion of variance $(p<0.05)$ are plotted. The percent variance explained by each factor is indicated using color in the plot and also shown in table $\mathrm{S} 1$

Figure 4.8:(A) A linear model was fitted for PC1 to extract features that are significant for phenology and year. The model included irrigation (Full, RDI, None), rootstocks (1103P, 3309C, Own and SO4), phenology (veraison, harvest), block (based on the position in the vineyard), and year (2017 and 2018). Only the factors which explained a significant portion of variance $(\mathrm{p}<0.05)$ are plotted. The percent variance explained by each factor is indicated using color in the plot. (B) A boxplot showing a metabolite feature differences between veraison and harvest

Figure 4.9: A linear model was fitted for(A) PC4, (C)PC7 and (E)PC8 respectively to extract features that are significant for rootstocks. On the right, boxplots showing differences in rootstocks due to (B)PC4, (D)PC7 and (F)PC8 respectively. The model included irrigation (Full, RDI, None), rootstocks (1103P, 3309C, Own and SO4), phenology (veraison, harvest), block (based on the position in the vineyard), and year (2017 and 2018). Only the factors which explained a significant portion of variance $(\mathrm{p}<0.05)$ are plotted. The percent variance explained by each factor is indicated using color in the plot.

Figure 4.10: A linear model was fitted for (A) PC12, (B)PC16 and (C) PC15 respectively to extract features that are significant for rootstocks. The model included irrigation (Full, RDI, None), rootstocks (1103P, 3309C, Own and SO4), phenology (veraison, harvest), block (based on the position in the vineyard), and year (2017 and 2018). Only the factors which explained a significant portion of variance $(p<0.05)$ are plotted. The percent variance explained by each factor is indicated using color in the plot. 195

Figure 4.11 Boxplots showing the concentration of free $\beta$-Damascenone in different (A) rootstocks and (B) irrigation regimes across various developmental stages and years. . 196 
Figure 4.12:Boxplots showing the concentration of total $\beta$-Damascenone in different (A) rootstocks and (B) irrigation regimes across various developmental stages and years .. 197

Figure 5.1: Heatmap of basic berry chemistry traits differences between different rootstocks grafted to Chambourcin and own-rooted Chambourcin.

Figure 5.2: Boxplots of berry chemistry traits (Titratable acidity (TA), Total soluble solids (brix), $\mathrm{pH}$, Tartaric acid, malic acid and citric acid) differences due to rootstocks (1103P, 3309Cand SO4) and own-rooted on Chambourcin scion. The values or concentrations are average of two years (2017 and 2018). The different letters in the boxplots represent significant difference (Duncan Multiple test)

Figure 5.3: Boxplots demonstrating impact of irrigation treatments (Full, RDI and None) on each berry chemistry traits (Titratable acidity (TA), Total soluble solids (brix), $\mathrm{pH}$, Tartaric acid, malic acid and citric acid). The different letters signify significant differences (Duncan Multiple test). The values are the average of two years.

Figure 5.4: Boxplots demonstrating yearly differences in concentration of the individual berry chemistry traits (Titratable acidity (TA), Total soluble solids (Brix), $\mathrm{pH}$ ), and organic acids (Tartaric acid, malic acid, and citric acid). The different letters signify significant differences between years (Duncan multiple tests)

Figure 5.5: Boxplots of midday leaf water potential (bars) and soil moisture content, berry weight per vine differences due to rootstocks (1103P, 3309Cand SO4), and ownrooted on Chambourcin scion in 2017 and 2018.

Figure 5.6: Boxplots of pressure chamber measurements(bars) and soil moisture content, berry weight per vine differences due to irrigation regimes(Full, RDI, and None) in 2017 and 2018.

Figure 5.7: Boxplots demonstrating differences in midday leaf water potential (bars) and soil moisture content, berry weight per vine and concentration of the individual berry chemistry traits (Titratable acidity (TA), Total soluble solids (Brix), $\mathrm{pH}$ ) and organic acids (Tartaric acid, malic acid, and citric acid) by blocks (position in the vineyard) for both 2017 and 2018 . 


\section{LIST OF TABLES}

Table 2.1: Mean concentration (ug/L) and standard error of mean (SE. mean) of free volatiles, total volatiles and Wine volatiles in Norton and Cabernet Sauvignon.

Table 2.2 Minimum, maximum and mean of concentration of free and bound volatiles in ug/L in F1 population of Norton and Cabernet Sauvignon.

Table 3.1 : Mean concentrations of wine volatiles in 2017 and 2018 as an Interaction effect between rootstocks and irrigation observed in wine volatiles in 2017 and 2018. The concentration was measured in $u g / L$.

Table 3.2 : Concentrations of Wine volatiles quantified $(\mu \mathrm{g} / L(\mathrm{ppb}))$ in 2017 and 2018 using GC-MS/MS. The p-values for significance for rootstocks and irrigations are also shown

Table 4.1: Mean concentrations of free, total and bound $\beta$-Damascenone in Chambourcin berries grafted to three different rootstocks and an own-rooted and subjected to three different irrigation regimes during two developmental stages for 2017 and 2018 seasons, Mt. Vernon, MO.

Table 4.2 Mean relative concentrations of free, total and bound Methyl Salicylate in Chambourcin berries grafted to three different rootstocks and an own-rooted and subjected to three different irrigation regimes during two developmental stages for 2017 and, USA2018 seasons, Mt. Vernon, MO,USA.

Table 4.3: Metabolic Features that are predictive between harvest and veraison for free and total volatiles, as calculated as correlation between harvest and veraison using Pearson's correlation. FDR correction was calculated to obtain the p-values..........225

Table 4.4: Metabolic Features that are predictive between 2017 and 2018 for free and total volatiles, as calculated as correlation between 2017 and 2018 features using Pearson's correlation. FDR correction was calculated to obtain the p-values...........227

Table 5.1: Mean concentrations of basic berry chemistry (Titratable acidity (TA), Total soluble solids (Brix), pH), organic acids (Tartaric acid, malic acid, and citric acid), and water potential of Chambourcin berries differed according to rootstocks and irrigation regimes..... 


\section{ABSTRACT:}

With increases in climate extremes resulting in more abiotic and biotic stress on crops such as grapes, it is essential to develop new cultivars that are more robust than the traditional Vitis vinifera but, with the flavor attributes consumers' desire. The interspecific hybrid cultivars developed from crossing V. vinifera with the wild Vitis species do possess cold hardiness and disease resistance, however, the aroma profile of these hybrids are generally less popular in comparison to the $V$. vinifera grapes. Developing an understanding of the aroma profile of the hybrid grapes is essential to understand how they differ from vinifera cultivars and how viticultural management practices impact their aroma composition. While earlier efforts have been made to determine the volatile profile of hybrid grapes and wines using targeted analysis, we opted for the more inclusive non-targeted metabolomics approach to investigate their differences. The objective of this dissertation is to investigate the aroma profile of two hybrid grapes Norton and Chambourcin, important to the Mid-Western United states, including Missouri, along with the differences during berry development. We also investigated the change in aroma profile of Chambourcin berries due to various viticulture operations, rootstocks, and irrigation using a metabolomics-based approach.

To understand consumer preference, differences in aroma between vinifera and interspecific hybrid grapes and wines need to be investigated so that the concentration of the key volatiles can be adjusted by various viticultural and enological practices to make better wine as well as develop cultivars that fit consumer preference better. In Chapter 2, we investigated berry and wine volatile differences between Norton, a popular 
interspecific hybrid cultivar in Missouri, and Cabernet Sauvignon, a popular Vitis vinifera cultivar known for its wine quality and cultivated worldwide. Berries were sourced from different locations, vintages, and ripening whereas, wines analyzed were commercial wines from different vintages and locations. The volatiles was analyzed using HS-SPMEGCMS and then processed using XCMS online to identify 924, 793, and 1064 metabolic features $(M(m / z) T$ (retention time in minutes)). Multivariate analysis of the significant features ( $p$-value $<0.05$ and fold change $>1.5$ ) demonstrated that the genome of the cultivar is the strongest factor driving differences in free, total and wine volatiles even though there is much variability in the environmental, year, viticultural factors in the berries and wine sampled. Many compounds were identified to be different between two cultivars and some related to plant defense including methyl salicylate and eugenol were found in higher concentrations in Norton. The identified compounds were used as markers to phenotype the F1 population derived from Norton and Cabernet Sauvignon cross. The F1 population demonstrated segregation for the identified compounds with some genotypes demonstrating higher or lower concentrations for the compounds. The future work is to map these volatile compounds using a genetic map to identify regions in genomes that are regulating them, which will accelerate the breeding of cultivars with desired traits from both cultivars.

Besides genetics, many viticultural factors such as irrigation management, rootstocks, sun exposure, and hedging have been found to play a pivotal role in manipulating the vine physiology and berry chemistry, thus impacting wine quality. We investigated the impact of rootstocks and irrigation treatments in berry and wine quality xvii 
using an experimental vineyard planted with Chambourcin own-rooted as well as grafted to three commercial rootstocks (1103P, 3309C, SO4) subjected to three irrigation regimes (full, RDI and none) in a complex experimental design. Since there is no published study on the volatile profile of Chambourcin grapes and wines, and since there is no predefined information on the volatiles that are important in this cultivar, we used a metabolomicsbased approach to extract as much information as possible. Chapter 3 of the dissertation aims to study the wine volatiles as impacted by rootstocks and irrigation in a comprehensive and unbiased way. Based on the multivariate analysis, rootstocks had the greatest influence on wine volatiles, but the assortment of compounds was modulated by the season and the irrigation regime. Own-rooted Chambourcin was found to differ from other root-systems for multiple volatile compounds such as Linalool, Ethyl Nonanoate, $\beta$ Damascenone, and TDN, whereas fewer differences were detected among grafted vines. Rootstocks were found to increase concentrations of compounds such as Linalool, while, $\beta$-Damascenone was found to be highest in own-rooted Chambourcin wines. Significant differences among irrigation regimes were also observed along with the interaction between rootstock and irrigation. $\beta$-Damascenone was higher in RDI than in other treatments. Finding significant differences in wine volatiles due to rootstocks and irrigation, we then investigated the berry volatiles during the course of its development also using the metabolomics-based approach.

Volatile compounds present in grapes, as well as aroma precursors, are crucial for wine quality, with their concentrations impacted by a complex interplay between the natural environment (soil conditions, climate, temperature), vineyard management $\mathrm{xviii}$ 
practices (irrigation, pruning, sun exposure) and vine genotypes, including the genomes of both the scion and the rootstocks. Grape-derived volatiles can be free volatiles that are present in grapes as well as aroma precursors which are present as glycosides in grapes and later released during the winemaking processes. Both free and bound volatiles are found to differ during berry development. We investigated the impact that rootstocks and irrigation have on free and total (free + glycosidase releasable) volatiles using the same Chambourcin experiment as described above. Phenology (ripening state) and year were found to be the most significant factors to impact berry volatiles, with berries being more similar in volatile composition at veraison than at harvest, confirming the commonly held view that secondary metabolism in berries reflects the various stresses the vine experiences during the growing season. Rootstocks and irrigation both had a subtle but significant impact on many free and total berry volatiles. Rootstocks were found to be a significant factor impacting free and total volatiles, differentiating own-rooted from grafted vines as well as differentiating between different rootstocks. The fact that rootstocks had only subtle effects on berry volatiles but a major impact on the relative concentration of wine volatiles raises the possibility that the principal manner by which rootstocks modulate wine volatiles may not be the concentration of berry metabolites. It is possible that other factors, such as grape-derived enzymes that modify or release volatile compounds during fermentation, are the ones through which rootstocks primarily influence wine quality. In the final chapter, the basic berry chemistry traits, organic acids, vine yield, and vine midday stem water potential as impacted by rootstocks and irrigation 
were investigated. Basic berry chemistry and organic acids significantly impact the flavor of the grapes and wines as well as the extraction of many volatiles from berries to wine.

The future work is to investigate the relationship between berry and wine volatiles to understand the predictability of wine quality. Also, correlating berry and wine volatiles with many other phenotypes that were collected concurrently as part of the bigger project will be performed. These include remote sensing, hyperspectral data, gene expression, leaf ionomics, leaf morphometrics, epigenetic regulation, and root microbiome that will unfold interesting details about the impact that each of those traits has on berry and wine quality. 


\section{Chapter 1: General Introduction and literature review}

Grape (Vitis species) is one of the most important fruit crops with a high economic impact on the global economy. With an economic impact of $\$ 219.9$ billion from grapes, wine, and its various components to the US national economy (https://wineamerica.org/impact/), the wine industry stands as an important industry in the United States providing around - 998,500 jobs. In Missouri, the wine industry contributes $\$ 3.2$ billion from the vineyard, winery, and tourism activities. The quality of the wine, its consumer demand and price is extremely dependent on the quality of grapes produced in the field which in turn depend on the genetics of grape cultivar used, various management practices used in the field, the environmental factors and the winemaking and fermentation decisions (Ferreira and Lopez 2019). The varietal aroma of the wine is the characteristic of the grapevine and is mainly attributed to the genetics of the grapevine. For example, the Muscat grapes have a varietal aroma that differentiates it from other grapevines. Many viticultural and winemaking operations can be adjusted to produce wine with the desired aroma. A large part of our understanding of the grapes and wine volatiles come from the studies based on European grapevines, Vitis vinifera. The effect of various viticultural and winemaking practices on the volatiles have been well studied to produce optimum quality wine from the grapes that are grown in different environmental conditions. However, in the Mid-Western United States, harsh winters followed by hot and humid summers do not permit commercial cultivation of many popular vinifera cultivars. These conditions necessitate the cultivation of cold-hardy and disease-resistant hybrid cultivars. These hybrid cultivars were developed by crossing European grapevines with the native North American grape species, mostly Vitis riparia, 
$V$. rupestris, $V$. aestivalis, and $V$. labrusca. While these grapes are economically important because of their tolerance to biotic and abiotic stress, the wines made from their fruit are different from the wines from the vinifera grapevines and, in most cases, not consumer preferred. Studies of some of these hybrid grapes have found that they have unique berry chemistry with high titratable acidity and low pH (Slegers et al. 2015). A handful of studies addressing the volatile composition of these grapes found them to possess a unique combination of volatiles that are different than those of vinifera grapes (Jogaiah et al. 2012; Sun et al. 2011b; Slegers et al. 2015; Slegers et al. 2017).

\section{Fruit and wine chemistry:}

The chemical composition of harvested grapes strongly influences the composition of must at the onset of vinification and the final quality of the bottled wine. After flowering and successful ovule fertilization, wine grape berries undergo a complex process of berry development involving two successive sigmoidal growth cycles characterized by three phases (CooMbe and McCarthy 2000). The initial phase begins with rapid cellular division in which the berry diameter increases and determines a berry's final size (Gouthu and Deluc 2015). Berries at this stage are small, green, hard, and highly acidic, mostly due to the accumulation of malic acid. Along with the accumulation of organic acids, this stage is also accompanied by the accumulation of tannins and hydroxycinnamates in berry skins and seeds. After the initial phase, which lasts approximately for 50 days following anthesis, there is a lag period determined by hormonal signaling and induction of auxin-response factor genes throughout the cluster (Kennedy 2002; Gouthu and Deluc 2015). Berry growth ceases, and berry acidity reaches maximum due to the continued accumulation of malic and tartaric acid. Veraison marks 
the beginning of the second growth cycle when berries begin, in an asynchronous manner, to accumulate sugars, anthocyanins, pigments, and additional phenols. After veraison, high concentrations of glucose and fructose accumulate in the berries along with a decrease in organic acid levels especially malic acid. This is accompanied by swelling and softening of berries due to water uptake, seeds maturation, and the accumulation of cations leading to an increase in $\mathrm{pH}$ (Coombe et al. 1987; CooMbe and McCarthy 2000; Ristic and Iland 2005). Berry ripening is also accompanied by synthesis of many aroma compounds and their precursors (terpenes, norisoprenoids, esters, and thiols) (Kennedy 2002). Berry chemistry during both phases of growth is highly influenced by genetic factors such as cultivar used and viticultural management decisions such as the use of rootstock, irrigation, disease management, soil nutrient management that implicate berry shading, vine growth and fruit load (Barnuud et al. 2014; Galletto et al. 2014; Main et al. 2002).

Sugars, $\mathrm{pH}$, and titratable acidity (TA) are the basic berry chemistry parameters used for determining the ripeness of berry. Sugars in grapes, mainly glucose and fructose are the primary sugars found in the grape berries and directly impact the final alcohol content of wine (Jackson 2008). Measured as soluble solids (in units of degrees Brix), glucose and fructose are predominant sugars in a Vitis vinifera berry, although the ratio of the two sugars may vary over the growing season or between cultivars (Kliewer 1965). Although present in trace amounts in most cultivars, sucrose has been measured at varying concentrations of total sugars in berries of interspecific hybrids (Liu et al. 2006). Sugar content in berries depends on various parameters such as vine vigor, crop load, vine water status, and a heat index of the growing season. 
The $\mathrm{pH}$ and TA of the berries are the measure of acidity in the berry and are an important parameter to determine ripening in berries. In general, wine grapes are harvested around an ideal $\mathrm{pH}$ of 3.5, lower titratable acidity of $5 \mathrm{~g} / \mathrm{L}$, and sugar accumulation of 20-24 degrees Brix, or approximately 200-240 g/L sugar. These conditions have the potential to create a balanced wine after fermentation, with 10-14 percent alcohol by volume (ABV).

\subsection{Organic acids:}

In grape juice and wine, organic acid composition plays an important role in organoleptic properties, microbiological control, and wine stabilization (Kliewer et al. 1967). Organic acids produce refreshing sour taste but also modifies the perception of taste, mostly sweetness. The acids released from vacuoles during crushing leads to acid hydrolysis of aroma precursors, which causes the release of many volatile compounds such as monoterpenes, C13 norisoprenoids, benzyl alcohol, phenolics, and 2phenylethanol. The major organic acids in grape juice are tartaric acid and malic acid, which constitutes 70-90\% of total grape acidity (Kliewer et al. 1967; Ruffner 1982b), while citric acid and succinic acid is found in low proportion. Unlike other acids, tartaric acid is unique to grapes and is rarely subject to microbial metabolism.

Tartaric acid accumulates in the berry skin and pulp prior to veraison, and its concentration per berry remains stable or slightly decreases throughout ripening in $V$. vinifera (Kliewer et al. 1967). In grapes with a lower amount of titratable acidity at the time of harvest, adjunct addition of tartaric acid can be made prior to fermentation to adjust the acid and sugar balance. However, care should be taken when making such 
additions as they can pose a problem of tartrate instability and precipitation later in the wine due to the formation of potassium tartrate and calcium tartrate.

Malic acid, the most prevalent acid in apples, is high in the grape berry early in the season due to de novo synthesis of malate via glycolysis and the TCA cycle (Ruffner 1982a). Following veraison, there is a rapid decrease in malic acid in berries due to a significant decrease in malic acid synthesis and a sharp increase in malic acid degradation via respiration. In wine, malic acid is readily metabolized by malic acid bacteria such as Lactobacillus and Pediococcus sp to lactic acid (Sweetman et al. 2009). This conversion of a diprotic acid to a monoprotic acid causes a drop in the perceived acidity of wine from a sensory perspective, which can further impact the perception of other volatile flavor and aroma compounds (Sweetman et al. 2009). This secondary malolactic (ML) fermentation is often associated with an increase in buttery, vanilla, and creamy flavors and aromas that may be desirable and pleasant in certain wines, but can also be considered flaws if the flavors and aromas overwhelm the consumer's palate. For most winemakers, unless the desired wine profile is strongly influenced by ML characteristics, grapes are harvested at the lowest malic acid concentration possible for that cultivar, towards the end of the growing season.

As the third most prevalent acid in grapes, citric acid can be metabolized by microbes as a tertiary carbon source after sugar and malic acid metabolism. Citric acid metabolism in winemaking is rarely induced because it results in acetic acid or diacetyl production, both of which can overwhelm the profile of the wine.

\subsection{The flavor profile of grapes}


The aroma is a crucial determinant of wine quality. Many studies have been done to understand the composition of volatiles in grapes and wines, however, due to interactions of the genetic factors with the environment, there is still a lot to be explored (Polaskova et al. 2008; Ilc et al. 2016). Wine aroma is a complex mixture of volatile organic compounds of a wide range of concentrations. Wine aroma can be derived from the grapes that define the varietal character of the wine, the fermentation aromas that are derived during fermentation and the aging aromas, also known as tertiary aromas that are extracted during chemical reactions occurring during aging (Ebeler 2001; Ebeler and Thorngate 2009; Rapp and Mandery 1986). The flavor is not the result of the activity of one or few individual volatiles but the interaction of many volatile compounds and matrix shapes how the volatile compounds taste or smell. Some individual aroma compounds have a dominant impact on wine aroma, however, the varietal differences in the aroma profile of different grape cultivars may be due to variation in the proportion of aroma compounds present, presence or absence of certain compounds, or an additive and synergistic effect of several aroma compounds.

\subsection{Free and bound volatiles in grapes}

Grape-derived free volatile compound and glycosidic bound volatile constitute the major aroma compounds in grapes and wine. Grape berries store most of the volatiles they produce as glycosidically bound aroma compounds in non-volatile form, which are the aglycones (free aroma compound) that are linked to a sugar moiety (Hampel et al. 2014). Although they do not contribute directly to wine aroma, they form a precursor pool that is released as volatiles during the fermentation process and hydrolytic activities during winemaking and by enzymatic hydrolysis in the mouth (Parker et al. 2017). They 
are the potential aroma contributors to the final wine (Ferreira and Lopez 2019). Aglycones constitute mostly terpenes, C13-norisoprenoids, volatile phenols, aromatic phenols, C6 compounds, aliphatic alcohols, aliphatic acids, benzenic, and phenolic acid derivatives. The varietal difference in the proportion of the glycosidic aroma compounds has been observed due to the variation in aroma biosynthesis genes among grape varieties (Ghaste et al. 2015; Rambla et al. 2016). Also, a wide range in the amount and the type of aglycone associated with the bound volatiles tend to differ between cultivars that render the varietal aroma distinction between varieties. Sometimes the varietal character or aroma is defined by a distinct chemical compound whereas in some cases they are the interaction result of a small group of such aroma compounds (Hjelmeland and Ebeler 2014; Robinson et al. 2009). The aroma glycosides are found to be present in higher proportion on the skin of the grapes in comparison to the pulp and juice, especially monoterpenes. C6 alcohols and furaneols were found higher in juice than skin.

Not all volatiles contribute to the aroma equally. Their impact depends on their concentration, as well as aroma intensity/odor detection threshold (the lowest concentration of a particular compound that can be detected by the human nose). In complex mixtures like wine, the odor threshold may not be the sole criterion for classifying important aroma as there can be significant synergistic and antagonistic effects among molecules. This interaction among molecules leads to the perception of molecules present at the sub-threshold level, whereas, some compounds can mask the perception of other compounds, so they remain undetected at supra-threshold concentrations (Ilc et al. 2016). Determination of the volatile composition of grapes also helps to predict the quality of wines. 


\subsection{Grape aroma molecules:}

\section{Terpenes:}

Terpenes, also known as isoprenoids, are one of the important secondary metabolites in plants important for the plant's resistance to diseases and pests. They are an important volatile constituent in grape berries, mostly present in the skin and little in the flesh as free and bound form. Free terpenes are volatile and odorous compounds and are present as monoterpenes and sesquiterpenes. Monoterpenes $\left(\mathrm{C}_{10}\right)$ and sesquiterpenes $\left(\mathrm{C}_{15}\right)$ are the most important aromatic volatiles in grapes (Black et al. 2015).

Monoterpenes are major compounds that impart floral and sweet notes to the wines made from aromatic cultivars like Muscat, Riesling, and Gewurztraminer due to their low sensory threshold (Schwab and Wüst 2015; Guth 1997). Among the 70 monoterpenes identified in grapes and wines, linalool, geraniol, nerol, $\alpha$-terpineol, citronellol, rose oxide, nerol oxide are the most important aroma compound, present in both free and glycosidic forms (Black et al. 2015). They are important for plants to attract appropriate pollinators and ensure reproductive success. Also, the volatile terpene emissions from the fruit attract seed dispersers. They also have antimicrobial activities and may deter the herbivores both above ground and in the root zone (Schwab and Wüst 2015). The de novo biosynthesis of terpenoids can be induced locally and systemically by herbivore feeding. Terpenoids are, therefore, able to provide rapid, but also herbivore-damage related signals to receiving plants (Holopainen and Blande 2012).

The concentrations of some groups of monoterpenes are tightly correlated because of their common metabolic origin. Linalool, a typical aroma of floral-scented wines, has a particularly rich oxidative metabolism and is converted to a variety of different 
compounds. Linalool oxygenases not only influence wine aroma by the formation of new compounds, but also by depletion of linalool (Ilc et al. 2016). Terpenes are biosynthesized from the primary metabolites in the cytosol through the Mevalonic acid (MVA) pathway and in the plastids through the 2C-methyl-D-erythritol pathway (MEP) pathway independently. In MVA, 3 molecules of acetyl CoA are combined stepwise to form mevalonic acid, then to isopentenyl diphosphate (IPP). In the MEP pathway, glyceraldehyde-3phosphate and pyruvate form the five carbon-intermediate 1-deoxy-Dxylulose-5-phosphate, which is reduced to 2-C-methyl-D-erythritol-4-phosphate (MEP). MEP is converted to dimethylallyl diphosphate (DMAPP). IPP and its isomer DMAPP forms the isoprene units which are the building blocks of the isoprenoids. IPP and DMAPP combine together to form geranyl diphosphate (GPP), which is a 10-carbon precursor for the synthesis of monoterpenes. GPP can further link with another molecule of IPP to form the 15-carbon compound Farnesyl diphosphate (FPP), which is the precursor of sesquiterpenes. In grapes, monoterpenes are primarily formed through MEP pathway and sesquiterpenes are formed through both MVA pathway and MEP pathway (Schwab and Wüst 2015). Metabolic flux is controlled by the reduction of 3-hydroxy-3methylglutaryl CoA (HMG-CoA) in the MVA pathway, whereas 1-deoxy-D-xylulose-5phosphate synthase (DXS) and 1-hydroxy-2-methyl-butenyl 4-diphosphate (HDT) are the key enzymes that control the flux of the MEP pathway (Schwab and Wüst 2015). The particular monoterpenes are synthesized by various terpene synthases (TPS) from the GPP. In grapevines, 69 putatively functional grapevine TPS gene family (VvTPS) have been found among which 39 are functionally characterized to synthesize monoterpenes, most of which are multiproduct enzymes accounting for many of the cyclic and acyclic 
monoterpene hydrocarbons and alcohols obtained through coupled isomerizationcyclization reaction sequence (Schwab and Wüst 2015).

Genetics plays a major role in terpenoids content. Cultivars such as Muscat, Riesling, and Gewurztraminer have a higher amount of monoterpenes in the berries and are known as aromatic cultivars (Black et al. 2015). These cultivars were found to have upregulation of many genes that code for many enzymes in the isoprenoid biosynthetic pathway. VvTPS genes are highly upregulated in these cultivars that play a role in the binding of GPP followed by the isomerization-cyclization reaction sequence to form various monoterpenes (Schwab and Wüst 2015). Besides genetics, growing conditions have a slight effect on monoterpenes. Biosynthesis of linalool was found to be inhibited by sunlight exclusion and was increased on re-exposure to the sunlight (Zhang et al. 2017). VvPNLinNerl, $V v C S b O c i, V v G t 7$, and $V v G T 17$ genes were mainly found to be responsible for monoterpene accumulation and sensitivity to sunlight. Additionally, the extraction of monoterpenes from the grape skin during winemaking also determines the final concentration of monoterpenes in the wine along with their acid-catalyzed rearrangements. Many canopy management practices such as leaf plucking and hedging to increase sun exposure have been found to increase both free and bound monoterpenes (Black et al. 2015). Many enzymes vital for the biosynthesis of monoterpenes and sesquiterpenes are downregulated in the water deficit grapevines relative to fully irrigated ones. Genes regulating the early steps of the MEP pathway, such as 1-deoxy-D-xylulose5 phosphate synthase (VviDXS1) and 1-deoxy-D-xylulose-5-phosphate reductoisomerase $(V v i D X R)$ were down regulated in water deficit grapevines. Water deficit was also found to modulate the expression of seven terpene synthases of the TPS family (Savoi et al. 
2016). Seven sesquiterpenes synthase genes were also found to be upregulated under water stress (Savoi et al. 2016). Induced synthesis of monoterpenes and sesquiterpenes occurs due to the action of methyl jasmonate as a defense response to herbivores and abiotic stress (Schwab and Wüst 2015). Transcripts related to hormone salicylic and jasmonic acid synthesis and auxin and brassinosteroid signaling were highly correlated with terpene synthesis under conditions of water deficit (Savoi et al. 2016).

\section{Norisoprenoids:}

Norisoprenoids are a group of carotenoid-derived compounds that have a low olfactory perception threshold and thus have a high sensorial impact on wine aroma (Mendes-Pinto 2009). These volatile organic compounds have a cyclic structure of 9, 10, 11 , or 13 carbon atoms. The C13-norisoprenoids are the most abundant norisoprenoids in grapes and are very important for red and white wine aromas. TCH $(2,2,6-$ trimethylcyclohexanone), $\beta$-damascenone, $\beta$-ionone, Vitispirane, Actinidiol, TDN $(1,1,6$ trimethyl-1,2-dihydronaphthalene), Riesling acetal and TPB (4-(2,3,6-trimethylphenyl) buta-1,3-diene) are the norisoprenoids identified in wine (Mendes-Pinto 2009). They are formed by direct degradation of carotenoids (C40) such as $\beta$-Carotene and neoxanthin, either directly by the dioxygenase cleavage or through enzymatic conversion to intermediate glycosides or precursors and then by acid-catalyzed conversion to aromaactive compounds (Winterhalter and Rouseff 2001; Robinson et al. 2014).

\section{Esters:}

Esters are mostly fermentation-derived volatiles that contributes to fruity notes of wine and other fermented beverages. Ethyl esters e.g. ethyl hexanoate and acetate esters e.g. isoamyl acetate are the major esters formed during fermentation and storage. While a 
negligible amount of esters are present in grapes, they are hydrolyzed during

fermentation. For example, the concentrations of ethyl hexanoate and ethyl octanoate are positively correlated to concentrations of their precursors, hexanoic acid, and octanoic acids, respectively. While hexanoic acid is predominantly a fermentation-derived product, non-negligible amounts are also found in grapes as well. The biosynthesis of hexanoic acid in grapes could thus influence the concentration of a typical fermentation product, ethyl hexanoate in wine. (Ilc et al. 2016)

\section{Aliphatic alcohols:}

Aliphatic alcohols are a diverse group of compounds that can originate both from grapes and yeast fermentation. C6-alcohols are a common group of plant volatiles with six carbon atoms and have a characteristic green, herbaceous aroma, reminiscent of leaves and cut grass and formed from the corresponding C6-aldehydes by alcohol dehydrogenases. C6-alcohols can be consumed by the yeast during fermentation to produce other compounds. High levels of short-chain fusel alcohols, formed from amino acid catabolism, can negatively impact the wine aroma (Ebeler and Thorngate 2009). Isoamyl alcohol is much higher in wine than in grapes.

\section{Aliphatic acids:}

Aliphatic acids are produced during yeast fermentation from primary metabolites: fatty acid-derived long aliphatic acids (C6 and above), amino acid-derived short and branched aliphatic acids, and sugar-derived aliphatic acid. The aroma of volatile fatty acids is generally unpleasant, sweaty, cheesy, goaty, and rancid. They are found to be present in both grapes and wines. They can be transformed into more pleasant-smelling compounds such as esters and lactones. 


\section{Hybrid grapes and their aroma}

Due to their disease tolerance capacity and the ability to tolerate biotic and abiotic stresses, the cultivation of the interspecific hybrids has increased, primarily in regions where traditional European grape cultivars cannot be grown profitably. The increased interest of grape growing the mid-western and northern United States where the summer is hot and humid and winter is cold, the growing of vinifera cultivars is problematic. Thus, interspecific hybrids are developed by crossing vinifera cultivars with Native American grapevines. The unique chemistry of these hybrid grapes such as higher titratable acidity, higher anthocyanin levels, and types rendering to the bluish tone and high $\mathrm{pH}$ as well as low degree of polymerization of the tannins makes them challenging for winemakers (Slegers et al. 2015; Slegers et al. 2017). Also, the challenging aspect of the hybrid grapes is the unique bouquet of the aroma or flavor. Recently, with the developing consciousness of consumers towards health and environmental sustainability contributing as economic drivers of profitability (Bisson et al. 2002b), the use of diseasetolerant hybrid grapevines and their wines have increased.

In cultivars derived from V. labrusca, new compounds, such as Methyl Anthranilate and O-Aminoacetophenone were discovered that were not found in $V$. vinifera. These compounds were identified as the main causal factors of the foxy and peculiar smell of labrusca varieties. Sun et al. (2011b) studied the volatiles in non-foxy hybrid grapes and compared it with vinifera grapes and found no unique compound contributing to the unique aroma of the hybrid grapes. Instead, they found a unique combination of various volatile compounds giving the definitive hybrid flavor to the grapes and wines. The foxy compounds methyl anthranilate and O-aminoacetophenone 
were not detected in the non- labrusca varieties, but there was a higher concentration of green and vegetal aromas in comparison to vinifera grapes (Sun et al. 2011b). Slegers et al. (2015) studied the free volatiles profile of berries and wines from the five red hybrid cultivars and found that there is variation in volatile composition between different hybrid cultivars derived from the same parent and suggested the ability to improve the quality of these wines by adjusting various viticultural and winemaking decisions based on the volatiles present in the grapes and wines. Mansfield et al. (2011) studied the impact odorant in the hybrid grape Frontenac and identified grape-derived compounds responsible for the varietal character of the Frontenac wines. Rice et al. (2019) also studied the effects of different harvest times on wine volatiles in interspecific hybrids, Frontenac Gris, and Brianna.

Previous research on hybrid grapes confirms that their berry chemistry is unique and cannot be explained by research centered on $V$. vinifera cultivars. With the climate in the United States changing, it is more important than ever to determine the berry and wine composition as well as the factors impacting the composition of cold-hardy and pest-resistant grape cultivars to produce a quality product that will enable the local markets to thrive. These hybrid grapes enable the industry to select for grapevines that are lower risk and lower maintenance by decreasing the need for frequent spray applications in the vineyard.

\subsection{Norton grapes and wines:}

The Norton grape is a complex interspecific hybrid wine grape derived from Vitis aestivalis, mostly cultivated in Southern and Midwestern United States including Missouri, Virginia, and Arkansas (Ambers and Ambers 2004; Ambers 2013). Norton is 
believed to be the result of an unintentional cross between a lost cultivar of $V$. labrusca and V. vinifera parentage and the native V. aestivalis grapevine (Ambers 2013). Besides its ability to make good wine, it has the ability to tolerate high and humid growing seasons along with low cold winters (Reisch et al. 1993; Main 2005). Additionally, Norton grapevine is generally resistant to multiple pests and disease pressures, including phylloxera, powdery mildew, downy mildew, and Pierce's disease (Reisch et al. 1993; Fung et al. 2008; Ali et al. 2011). Norton leaves have been found to accumulate a high level of salicylic acid and SA-related defense genes in comparison to Cabernet Sauvignon which may have contributed Norton grape with a robust innate defense system against pathogens (Fung et al. 2008). The presence of elevated levels of pathogenesis-related transcripts and stilbene synthase genes in Norton berries also suggests the developmentally regulated resistance of Norton (Ali et al. 2011). Norton grapes are found to be rich in total phenolic, anthocyanin, and flavonoid content (Hogan et al. 2009). The Norton grapevine is vigorous (1.8-2.0 lbs pruning weight per vine), produces small blueblack berries in characteristically tight, small clusters resulting in a low crop yield (mean cluster weight 65-89 g; 9-12 tons per acre) (Main 2005; Jogaiah et al. 2013).

\subsection{Chambourcin grapes and wines}

Chambourcin is phylloxera- and humidity-tolerant French hybrid grape, derived from a cross between Seyve -Villard 12-417 with Chancellor. It was developed by Joanne Siebel in the 1960s and was only commercially available since the 1970s in the Loire valley. The Chambourcin wine has a distinct black cherry, black pepper, crushed gravel, wet loam, and chocolate aroma with high acidity and low tannin. In general, Chambourcin wine has dark colors with intense spicy aromas and flavors with notes of 
cherries and plums, and some noticeable herbal traits as well

(https://worldsbestwines.eu/grapes/chambourcin/).

\section{Plant Metabolomics}

Metabolites are the final products of the gene expression and are the chemical phenotype of an organism. Metabolomics is the comprehensive profiling of the complete set of all metabolites of an organism in an unbiased way (De Vos et al. 2007; SchrimpeRutledge et al. 2016). Comprehensive identification and quantification of all intra- and extracellular metabolites (generally, metabolites with a molecular mass lower than 1000 Da) to find explanations of biological phenomena under certain conditions requires careful analysis and interpretation of the data. Due to a huge diversity of plant metabolites ranging up to 20,000 metabolites in a single plant and complexity arising from this, there is no single method that can simultaneously provide a comprehensive overview of the metabolome. Instead, a combination of different analytical techniques is applied to obtain as much information as possible (De Vos et al. 2007; Gika et al. 2014; Saito and Matsuda 2010; Schrimpe-Rutledge et al. 2016).

The major techniques applied in metabolomics research are (i) targeted analysis, (ii) metabolite fingerprinting, (iii) metabolite profiling. The targeted analysis aims at accurate quantitative measurements of the selected few metabolites that are known to be important

in a biological system. Metabolite fingerprinting aims to screen the metabolome in a high throughput manner to discriminate samples of different biological status or origin and does not include the identification or quantification of the metabolites. In contrast, metabolite profiling aims at comprehensively identifying as many metabolites as possible 
in a given system at a given time along with identification and quantification (or semiquantification) of specific metabolites belonging to the same class of compounds or a specific pathway (Commisso et al. 2013; Flamini et al. 2014; Saito and Matsuda 2010; Schrimpe-Rutledge et al. 2016).

\subsection{Targeted and untargeted metabolomics}

The untargeted metabolomics approach tries to gather as much information on as many metabolites as possible in each sample. This type of approach is often qualitative and particularly important when no primary or secondary metabolite is defined or available. The untargeted metabolomics approach is hypothesis-generating, while the targeted approach is usually applied to validate discoveries of a hypothesis-generating study. There is a possibility to detect unexpected changes in metabolites using an untargeted approach, as this approach aims to maximize the number of metabolites detected. Multiple analytical approaches are combined to maximize the number of metabolites detected, as no single analytical method can detect all the compounds present in the living system. Sample preparation in untargeted studies involves the extraction of the metabolite from the biological sample into a suitable solvent and then analyzed using an appropriate analytical method (De Vos, Moco et al. 2007, Schrimpe-Rutledge, Codreanu et al. 2016). The main feature of this approach is the conversion of the chromatographic information into coordinates in the feature space using mass to charge ratio, retention time, and signal amplitude and then alignment across all the samples (De Vos et al. 2007). Unlike a targeted approach where standards are used to identify mass peaks as the primary step, this approach enables us to identify the metabolites that would have been missed if only the targeted approach has been used. Multivariate statistics 
enable us to identify the differential mass peaks correlating with a specific trait. The features or traits are then identified using accurate mass, MS/MS fragmentation, and then confirmed with standards when available. Thus, mass peak identification using standards is done later for the confirmation of the identified compounds using features (SchrimpeRutledge et al. 2016; De Vos et al. 2007). Untargeted metabolomics approach aims to cover as many metabolites as possible present in a biological system, this helps to uncover previously unknown metabolites present in the system as well as to understand many regulatory functions of in metabolic pathways. This approach is especially valuable to study understudied biological systems or treatment impact on those systems as it is necessary to first be inclusive in the initial selection of analytes of interest.

\subsection{Untargeted Metabolomics Workflow}

Metabolomics is a complex multidisciplinary approach that requires knowledge in life sciences, analytical chemistry, organic chemistry, chemometrics, and informatics. The general workflow of untargeted metabolomics studies includes biological sample design, sample collection and preparation, raw data acquisition via different analytical platforms, data processing, and pre-treatment, metabolite identification, statistical analysis for potential biomarker identification, confirmation and quantification of the identified biomarkers, and finally the biological interpretation of the results using pathway and network analysis approaches. 


\subsection{Solid-phase microextraction (HS-SPME):}

SPME is a solvent-free sample pre-treatment and extraction technique that encompasses sampling, extraction, concentration, and sample introduction into one step. It consists of a small fused silica fiber coated with a polymeric phase, which is mounted in a syringe-like device. The principle of SPME is absorption or adsorption of the analytes into a fiber phase until the equilibrium is reached between sample matrix and fiber coating. After equilibrium is reached, the absorbed compounds are thermally desorbed by exposing the fiber into the injection port of a GC or dissolved in an organic solvent for further analysis with GC, LC, or CE. Compared to other sample extraction methods such as solid-phase extraction, solvent extraction, SPME has the advantages of being simple, fast, inexpensive, no-solvent requirements, and limited sample manipulation. It can be easily interfaced with gas chromatography. Depending on the type of sample, various parameters are optimized for higher extraction efficiency such as extraction phase chemistry, extraction mode, agitation method and time, sample modification ( $\mathrm{pH}$, ionic strength, organic solvent content), sample temperature, extraction time and desorption conditions ( $\mathrm{Xu}$ et al. 2016).

\subsection{Analytical Methodology:}

Due to the complexity of biological samples in metabolomics research as well as the low concentrations of individual compounds, extremely high-resolution and sensitive analytical techniques are required. Mass spectrometry and nuclear magnetic resonance spectroscopy are the two key analytical techniques used in metabolomics. MS-based approaches can be used with prior chromatographic or electrophoretic separation such as 
gas chromatography (GC), liquid chromatography (LC) or capillary electrophoresis or as direct MS without chromatographic separation (Schrimpe-Rutledge et al. 2016).

Mass spectrometers operate by ion formation, separation, and subsequent detection of ions based on their mass-to-charge $(\mathrm{m} / \mathrm{z})$ ratio. Molecules can be ionized by electron ionization, a hard ionization technique where molecules are broken into several fragments. Alternatively, soft ionization, which does not introduce significant fragmentation of the molecular ions, can be used, e.g. chemical ionization (CI), atmospheric pressure chemical ionization (APCI), electrospray ionization (ESI) or matrix-assisted laser desorption/ionization (MALDI) The most frequently used ionization in metabolomics are EI (GCMS) and ESI (LCMS) (Schrimpe-Rutledge et al. 2016) Depending on the applied type of ionization technique, positive, negative or both types of ions can be generated (Gross 2006).

The most common single configuration mass analyzers are linear quadrupole $(\mathrm{Q})$, ion trap, time of flight (TOF), and Fourier transform ion cyclotron resonance, with increasing order of price, resolution, and sensitivity. The combination of different mass analyzers, e.g., Q-TOF or combination of same mass analyzers (QQQ), are also available depending upon accuracy and sensitivity required (Gross 2006).

GC is an analytical platform that separates the complex mixture in gaseous form based on their vapor pressure. Only compounds that are volatile and thermally labile can be separated using GC. Large molecules or small molecules with polar groups tend to have lower volatility, leading to extremely long retention times or non-elution of the compounds, decomposition, or asymmetric chromatographic peaks when analyzed utilizing gas chromatography (Gross 2006; Poole 2010). Therefore, such compounds 
require derivatization of their functional groups to increase thermal stability and volatility in GC analysis. The most commonly used stationary phases of capillary GC columns are dimethylpolysiloxane, $5 \%$ diphenyl/95\% dimethyl polysiloxane, and trifluoropropylmethyl polysiloxane (Poole 2010).

In the last 30 years, many different methods for studying the aroma compounds in fruits and wines have been developed using a targeted approach, mostly using GC coupled with different detectors, with various sample preparation techniques. Nowadays, the holistic approach of metabolomics leads to the development of methods with extended coverage to include different classes of compounds in a single run. Since many volatile and semi-volatile compounds are present in trace amounts, the development of the sensitive analytical method is necessary to measure odor concentrations ranging from $\mathrm{ng} / \mathrm{L}$ to $\mathrm{mg} / \mathrm{L}$. A triple quadrupole mass spectrometer operating in selected reaction monitoring is the method of choice in quantitative metabolic studies. It has good sensitivity and good dynamic range, allowing quantification of the wide range of metabolites in varying concentration range. GC/MS/MS is commonly used in food analysis to analyze multiresidual pesticides in vegetables and fruits. The multiple reaction monitoring with a selection of two transition ions, one for quantification and one for confirmation, provided excellent selectivity and sensitivity.

\subsection{Data pre-processing and pre-treatment:}

Data pre-processing and pre-treatment is an important step in metabolomics data analysis to improve the biological interpretability of the data. After data acquisition, preprocessing steps are used to transform raw instrumental data into clean data. This includes deconvolution (separation of overlapping signals into individual chemical 
peaks), peak picking, alignment (correcting shifts in retention times between different runs), baseline correction, and missing value imputation (Tautenhahn et al. 2012; Schrimpe-Rutledge et al. 2016; Arapitsas et al. 2020). The chromatographic information is converted into coordinates in the feature space using mass to charge ratio, retention time, and signal amplitude and then aligned across all the samples (De Vos et al. 2007). To maintain the integrity of the data, missing data are imputed by means or medians of the metabolite across different samples or employing the nearest neighbors (Xia and Wishart 2016). Several software tools for data processing are available, including commercial software, such as MassHunter Mass Profiler Software (Agilent Technologies, Santa Clara, CA, USA), Sieve (Thermo Fisher Scientific, Waltham, MA, USA) or metAlign (Plan Research International, Wageningen, The Netherlands), and free software, such as XCMS online (Tautenhahn et al. 2012), MZmine (Katajamaa et al. 2006), Chrompare (Want and Masson 2011).

Additional pre-treatments steps are also required before performing multivariate statistical analyses. This step includes the normalization of the data, data transformation, centering, and scaling. For example, normalization with an internal standard is popular to remove unwanted systematic variation between samples and to make observations more directly comparable (Want and Masson 2011; Schrimpe-Rutledge et al. 2016). Similarly, centering the data around zero mean helps to remove dominance between high and low abundant metabolites to focus on the relevant variation between samples. By application of scaling methods such as autoscaling, Pareto scaling, and range scaling, the masking of low abundant metabolites by high abundant metabolites can be avoided. Similarly, log transformation can be applied to approximate the normal distribution and to correct 
heteroscedastic noise. The type of data pre-treatment depends on the data and the type of biological information required from the data (Gowda et al. 2014; Schrimpe-Rutledge et al. 2016).

\subsection{Statistical analysis:}

The complex and huge dataset from the metabolomics experiment needs the use of appropriate statistical tools, including multivariate and univariate methods to extract relevant information and to better understand and interpret the underlying structure of the data.

\subsection{Multivariate analysis:}

This includes both unsupervised and supervised methods. Principal component analysis (PCA) is one of the most popular unsupervised methods widely used in multivariate analysis of metabolomics data. Metabolomics data is mostly characterized by a large number of variables, mostly greater than the number of samples (Otto 2016). PCA reduces the dimensionality of the data into a few principal components (PC) that represent most of the variation of the data. This is achieved by a linear transformation of all variables into a new orthogonal coordinate system called principal components so that the greatest variance of the data is found on the first axis (PC1) and the second-largest variance on the second axis (PC2) (James et al. 2013; Otto 2016). The resultant data is visualized in terms of scores plot and loadings plot. The scores plot shows the distribution of data points or samples whereas, the loading plot reflects the distribution of variables or metabolites driving differences between samples or data points (James et al. 2013). Besides PCA, cluster analysis, hierarchical cluster analysis, K-means clustering, tree diagrams are also employed as unsupervised approaches in metabolomics. These 
methods group and visualize samples depending on their intrinsic similarities using distance metrics such as Euclidean distance, Manhattan distance, or correlation (James et al. 2013).

Although unbiased dimension reduction of the huge metabolomics dataset can be achieved using an unsupervised PCA approach, it will only reveal a group structure as long as the between-group variation is sufficiently more pronounced than the withingroup variation (James et al. 2013). On the other hand, supervised methods such as partial least square analysis (PLS), partial least square discriminant analysis (PLS-DA), orthogonal partial least square discriminant analysis (OPLS-DA), or soft independent modeling of class analogy (SIMCA) can be used to maximize group separation according to pre-defined categories (Liland 2011). PLS-DA is one of the most extensively applied data analysis techniques in metabolomics, where samples are classified into pre-defined groups, and then used to maximize the group separation according to class belonging (James et al. 2013; Otto 2016). Supervised methods like PLS and PLS-DA force the separation between experimental groups in score plots, thereby normally resulting in a better class separation compared to PCA. Thereby, such supervised methods tend to easily overfit models and overestimate their predictability, i.e. models can just by accident result in a good classification of the groups (Otto 2016). Therefore, the reliability and robustness of such models require rigorous validation, e.g. by permutation tests (random permutation of group membership) or by K-fold cross-validation (splitting the dataset K-times into a training and a test set) (Otto 2016; Liland 2011).

\subsection{Univariate Analysis}


Parametric and non-parametric procedures are available to determine whether a metabolite shows statistically significant differences between the investigated groups. The decision which univariate test is the most suitable depends on several statistical properties of the data which have to be tested in advance, including normality (e.g. Shapiro-Wilk or Kolmogorov-Smirnov test), homogeneity of variances (e.g. Levene's or Bartlett's test), and independence. Two events are statistically independent if the occurrence of one event does not affect the probability of occurrence of another event. Parametric tests such as paired or unpaired t-test (two-group comparisons) and analysis of variance (ANOVA) followed by multiple pairwise comparisons with posthoc tests such as Tukey's honestly significant difference (HSD) or Fisher's least significant difference (LSD) test are conducted for normally distributed metabolites with homogeneity of variance. Non-parametric tests such as Mann-Whitney U-test (unpaired groups), Wilcoxon signed-rank test (paired groups), and Kruskal-Wallis test for multiple comparisons can be used for non-normal data. Alternatively, non-normally distributed data can be transformed to normal or near to normal data using different data transformations such as log transformation, square root, or arcsin transformation (James et al. 2013).

In metabolomics studies, normally hundreds of metabolites are determined, which needed to be tested for significance (De Vos et al. 2007; Schrimpe-Rutledge et al. 2016; Liland 2011). However, when more hypotheses are tested on the same data set, the probability of wrongly rejecting the null hypothesis by chance increases that can lead to a substantial increase in the number of false positives (Type 1 error). For a single test, an $\alpha$ level of 0.05 means that the probability of making a Type 1 error is equal to $\alpha=0.05$ and 
the probability of not making a Type 1 error is consequently equal to $1-\alpha=0.95$. For multiple tests, the probability of at least one Type 1 error increases. In general, the probability to find at least one Type 1 error in $\mathrm{c}$ independent tests on the same data set is equal to 1-(1- $\alpha)^{\mathrm{c}}$ (Otto 2016). Therefore, in the case of multiple hypothesis testing, the significant levels need to be protected against an increasing probability of getting false positive results by adjusting the significance level to the number of tests performed, e.g. by Bonferroni correction or by controlling the false discovery rate (ratio of the number of false positives to the number of positives) (Otto 2016; Schrimpe-Rutledge et al. 2016). Bonferroni correction is the most conservative and most commonly used method in metabolomics studies (Noble and Ebeler 2002; Liland 2011).

\section{Development and use of rootstocks in viticulture:}

The replacement of the entire root system of a plant by a different root system genotype is known as grafting and is a very popular concept in horticulture including grapevines (Mudge et al. 2009). Approximately $90 \%$ of grapevines grown in the world belongs to only one species, $V$. vinifera (Alessandrini et al. 2017; This et al. 2006). The concept and practice of grafting a vinifera variety on a disease-resistant and stresstolerant rootstock have been popular in viticulture since the introduction of phylloxera (Daktulosphaira vitifoliae Fitch) to European vineyards in the $19^{\text {th }}$ century. The aphidlike insect in the soil infests the roots of susceptible Vitis vinifera grapevines, resulting in rapid defoliation and collapse of the root system, ultimately leading to the death of the entire vine. This led to the destruction of a quarter to a third of all grape vineyards planted in France (Mudge et al. 2009). Due to the continued work of many scientists including C.V. Riley, an American entomologist, the pest was identified to be tolerant to 
the American grape species due to the coevolution of the pest with the host that rendered resistivity to the pest (Mudge et al. 2009). The solution to this problem was identified in which the European cultivars were grafted onto resistant native American grape species. Crosses of diverse North American Vitis species (mostly V. berlandieri, V. rupestris, and V. riparia) are mostly used as rootstocks due to their resistance to phylloxera and their ability to adapt to varying soil conditions which is used to date. Besides, rootstocks are also used for their resistance or tolerance to nematodes, adverse soil conditions such as drought, waterlogging, high or low $\mathrm{pH}$, or salinity and their ability to influence vine vigor and fruit ripening (Nelson et al. 2015; JONES et al. 2009b; Cousins 2005; Reynolds and Wardle 2001).

\section{Rootstocks effect on grapes and wine chemistry:}

The alteration of various physiological processes in the scion due to rootstock has a profound effect on vigor or biomass accumulation, yield, berry composition, fruit quality, fruit phenolic compounds, ion concentration and response to abiotic stresses and salinity (Wooldridge et al. 2010; Harbertson and Keller 2011; Ezzahouani and Williams 1995; Nelson et al. 2015; Migicovsky et al. 2019; Main et al. 2002). However, genetic differences between rootstock cultivars can influence water availability to the scion, root dynamics, N uptake, canopy biomass, and finally fruit composition (Stockert et al. 2013; Tramontini et al. 2013). Although scion is found to have the most influence on the fruit composition (Keller et al. 2012), rootstocks may alter fruit composition and volatile profile indirectly by influencing scion vigor, canopy configuration, yield formation, and nutrient uptake. It is also found through the transcriptomic studies that grafting with rootstocks had a profound effect on the scion gene expression in the shoot apex (Cookson 
and Ollat 2013; Migicovsky et al. 2019). The use of rootstocks provides an improvement in plant vigor, yield, and some characteristics of the fruit, such as $\mathrm{pH}$, acidity, and soluble solids.

A lot of research has been done to study the impact of various rootstocks on the vine growth and fruit composition of the scion (Berdeja et al. 2014; Berdeja et al. 2015; Cookson and Ollat 2013; Harbertson and Keller 2011; JONES et al. 2009b; Migicovsky et al. 2019; Tramontini et al. 2013; Wooldridge et al. 2010), but the research on the impact of rootstocks on the grape and wine aroma is scarce (Koundouras et al. 2009). Even more limited are the studies of the effect of rootstocks on the phylloxera-tolerant hybrid grapes and wines, especially on the aroma. Wine flavors and aromas are crucial to the wine quality and therefore, our study aimed at investigating the effect of different rootstock genotypes grafted onto the same scion on the volatile aroma compounds of grapes and wines.

Although rootstocks are widely used with wine grape cultivars in Europe, California, and Australia, the majority of hybrid grapes produced in the Eastern and Midwestern United States are not grafted to rootstocks (Thomas et al. 2017). 'Couderc 3309' hybrid grape (3309C) is a commonly used rootstock worldwide developed by Georges Couderc in France in 1881. This rootstock performs well in the Midwestern United States, imparting winter hardiness and flood tolerance to hybrid scions (Striegler and Howell 2015; Striegler et al. 1993) as demonstrated in many fields and greenhouse studies. Rootstock effect on hybrid genotype scion has also been shown to impact vine vigor and yield (Sabbatini and Howell 2013). Varying degrees of rootstock effect has also been 
observed on juice characteristics of hybrid grapes and finished wines (Main et al. 2002;

Striegler et al. 1993; Thomas et al. 2017; Gu et al. 2005; Striegler and Howell 2015).

\section{Irrigation effect on grapes and wine chemistry:}

Water is critical for growth and development. Water stress during the critical growth stages of a plant is known to cause a negative effect on growth, reproduction, and thus on yield. Water uptake is driven by transpiration through the leaf stomata, while the internal water movement is largely driven by pressure potential gradients. Water stress during fruit set can result in low fruit set and smaller berry size, whereas, excessive water results in too much vegetative growth. However, medium water stress is known to have a beneficial effect especially on the quality of the grapes and wine aroma perspective. Water stress leads to reduced water uptake and reduced shoot growth and yield leading to smaller berries with a higher concentration of total phenolic substances and anthocyanins which are important for wine quality. Thus, water stress does not only impart negative effects because regulated water stress can improve grape and wine composition through controlling vine growth (Harbertson and Keller 2011; Keller et al. 2012). Medium soil water deficit was found to correlate with positive wine sensory attributes with higher intensity of flavor and aroma attributes in deficit irrigation (Koundouras et al. 2009; Bindon et al. 2007). Wines made from vines under deficit irrigation were associated with descriptors like more fruity and less vegetal characters. This may be due to the activation of alternative biosynthetic pathways that leads to greater carbon flux that increases amino acids (precursors of esters in wines) and in carotenoids, resulting in more fruity aromas. Also, vine water status was in a good position in deficit irrigation in Chardonnay grapes. (Balint and Reynolds 2017). Limited water availability reduces vine vigor, increases 
berry sun exposure and temperature, accelerates the degradation of carotenoids, and enhances the formation of norisoprenoids compounds (Qian et al. 2009). Limited water availability was also found to alter the concentration of carotenoids and their norisoprenoids derivatives in berries (Bindon et al. 2007). The concentration of $\beta$ Damascenone, TDN, and $\beta$-ionone increased in vines treated with deficit irrigation. Irrigation strategy can have an impact on the formation of glycosylated precursors and C13 norisoprenoids. Norisoprenoids like $\beta$-Damascenone contribute to complex wine aroma, has a very low aroma threshold (0.05 ug/L in ethanol). Deficit irrigation was found to result in a higher concentration of $\beta$-Damascenone in wines compared to full irrigation.

The root systems provide water and mineral nutrients to the shoot system, while the shoot system nourishes the overall plant through photosynthetic assimilates. While the scion variety contributes to the fruit composition and quality in the grafted plants (Harbertson and Keller 2011), the rootstocks can alter the characteristics of the shoot, which provides an opportunity to manipulate and change varietal traits to improve grape and wine quality. Berdeja et al. (2014) studied yield and berry composition (sugars, organic acids, free amino acids, and anthocyanins) in grafted Pinot Noir and found no significant effect of water stress on yield, sugar, and organic acid concentration, but increase in anthocyanin concentration due to water stress (Berdeja et al. 2014). There was only a subtle difference due to rootstocks on scion berry composition.

Grapevine species which tend to be more tolerant to water stress have greater water use efficiency and requires less amount of water. Isohydric vines maintain a strict water balance by maintaining higher midday leaf water potential through a reduction in 
stomatal conductance. This behavior is mostly observed in plants in wet climates and is more prone to xylem cavitation during water stress. Anisohydric vines exhibit more variable leaf water potential and have their stomata open longer, resulting in greater photosynthetic activity even in times of drought, and vines are less susceptible to xylem cavitation. These plants have a higher root to shoot ratio which aids in drought avoidance. $V$. vinifera cultivars are generally tolerant to some levels of drought stress, whereas, American species such and $V$. riparia and $V$. rupestris are not tolerant to drought. $V$. berlandieri and V. cordifolia are the American grape species that have developed tolerance to drought.

Prolonged water stress can have a negative impact on the growth and development of plants including grapevines. Excess irrigation and water availability may contribute to increased vegetative growth leading to shading of grape clusters and the development of many fungal pathogens and diseases. On the other hand, mild to moderate water stress through regulated deficit irrigation can bring a positive impact on the fruit and quality of the grapevine through reduction of vine excessive vigor and increasing berry and wine quality through the formation of many useful metabolites. Deficit irrigation has been found to reduce stomatal conductance but maintain photosynthesis, thereby increasing water use efficiency in grapes than fully irrigated vines (Chaves et al. 2007).

\section{Midday Stem Water Potential:}

The water status in plants was determined by measuring the midday stem water potential by the use of a pressure chamber. Pressure chamber measurements on nontranspiring leaves reflect the water potential of the xylem in the stem to which the leaf is 
attached (Meinzer et al. 2001). It measures the capacity of the cells to retain water by pressurizing a leaf with a neutral gas. The greater the pressure required to exude water from the stem, the more water-stressed is the plant. The measurements from pressure bomb are expressed in bar or MPa and the midday stem water potential is expressed as a negative value. The non-transpiring leaf function as the tensiometer which estimates the xylem pressure of the stem and remaining nearby leaves from its balance pressure if water columns are continuous throughout the plant and if local variations in xylem tension are negligible.

Midday stem water potential ( $\Psi$ stem) is the most widely used measurement for assessing vine water status and for depicting the relationships between berry traits and water stress. Studies have found that midday stem water potential measured before veraison was significantly and positively correlated to berry weight and must titratable acidity, whereas it was negatively correlated to must TSS, malic acid concentration, and wine $\mathrm{pH}$, tartaric acid, anthocyanins, and total phenolic index (TPI). Istem was positively correlated with berry weight and must titratable acidity and negatively correlated to TSS (Mirás-Avalos and Intrigliolo 2017).

\section{References}

Alessandrini M, Battista F, Panighel A, Flamini R, Tomasi D (2017) Effect of pre-bloom leaf removal on grape aroma composition and wine sensory profile of Semillon cultivar. J Sci Food Agric. doi:10.1002/jsfa.8638

Ali MB, Howard S, Chen S, Wang Y, Yu O, Kovacs LG, Qiu W (2011) Berry skin development in Norton grape: distinct patterns of transcriptional regulation and flavonoid biosynthesis. BMC plant biology 11 (1):1 
Ambers CP (2013) A historical hypothesis on the origin of the Norton grape. Journal of Wine Research 24 (2):85-95. doi:10.1080/09571264.2012.747087

Ambers RK, Ambers CP (2004) Dr. Daniel Norborne Norton and the origin of the Norton grape. Am Wine Soc J 36 (3):77-87

Arapitsas P, Ugliano M, Marangon M, Piombino P, Rolle L, Gerbi V, Versari A, Mattivi F (2020) Use of Untargeted Liquid Chromatography-Mass Spectrometry Metabolome To Discriminate Italian Monovarietal Red Wines, Produced in Their Different Terroirs. Journal of Agricultural and Food Chemistry. doi:10.1021/acs.jafc.0c00879

Balint G, Reynolds AG (2017) Irrigation level and time of imposition impact vine physiology, yield components, fruit composition and wine quality of Ontario Chardonnay. Scientia horticulturae 214:252-272

Barnuud NN, Zerihun A, Gibberd M, Bates B (2014) Berry composition and climate: responses and empirical models. International journal of biometeorology 58 (6):1207-1223

Berdeja M, Hilbert G, Dai ZW, Lafontaine M, Stoll M, Schultz HR, Delrot S (2014) Effect of water stress and rootstock genotype on Pinot Noir berry composition. Australian Journal of Grape and Wine Research 20 (3):409-421. doi:10.1111/ajgw.12091

Berdeja M, Nicolas P, Kappel C, Dai ZW, Hilbert G, Peccoux A, Lafontaine M, Ollat N, Gomès E, Delrot S (2015) Water limitation and rootstock genotype interact to alter grape berry metabolism through transcriptome reprogramming. Horticulture research 2:15012

Bindon KA, Dry PR, Loveys BR (2007) Influence of Plant Water Status on the Production of C13-Norisoprenoid Precursors in Vitis vinifera L. Cv. Cabernet Sauvignon Grape Berries. Journal of Agricultural and Food Chemistry 55 (11):4493-4500. doi:10.1021/jf063331p

Bisson LF, Waterhouse AL, Ebeler SE, Walker MA, Lapsley JT (2002) The present and future of the international wine industry. Nature 418 (6898):696-699. doi:10.1038/nature01018 
Black C, Parker M, Siebert T, Capone D, Francis I (2015) Terpenoids and their role in wine flavour: recent advances. Australian journal of grape and wine research 21:582-600 Chaves MM, Santos TP, Souza Cd, Ortuño M, Rodrigues M, Lopes C, Maroco J, Pereira JS (2007) Deficit irrigation in grapevine improves water-use efficiency while controlling vigour and production quality. Annals of Applied Biology 150 (2):237-252

Commisso M, Strazzer P, Toffali K, Stocchero M, Guzzo F (2013) Untargeted metabolomics: an emerging approach to determine the composition of herbal products. Computational and structural biotechnology journal 4 (5):e201301007

Cookson SJ, Ollat N (2013) Grafting with rootstocks induces extensive transcriptional reprogramming in the shoot apical meristem of grapevine. BMC Plant Biology 13 (1):147. doi:10.1186/1471-2229-13-147

Coombe B, BOVIO M, SCHNEIDER A (1987) Solute accumulation by grape pericarp cells: v. relationship to berry size and the effects of defoliation. Journal of Experimental Botany 38 (11):1789-1798

CooMbe BG, McCarthy M (2000) Dynamics of grape berry growth and physiology of ripening. Australian journal of grape and wine research 6 (2):131-135

Cousins P (2005) Evolution, genetics, and breeding: viticultural applications of the origins of our rootstocks. Grapevine Rootstocks: Current Use, Research, and Application:1

De Vos RC, Moco S, Lommen A, Keurentjes JJ, Bino RJ, Hall RD (2007) Untargeted large-scale plant metabolomics using liquid chromatography coupled to mass spectrometry. Nature protocols 2 (4):778

Ebeler SE (2001) Analytical Chemistry: Unlocking the Secrets of Wine Flavor. Food Reviews International 17 (1):45-64. doi:10.1081/fri-100000517

Ebeler SE, Thorngate JH (2009) Wine chemistry and flavor: looking into the crystal glass. J Agric Food Chem 57 (18):8098-8108. doi:10.1021/jf9000555 
Ezzahouani A, Williams LE (1995) The influence of rootstock on leaf water potential, yield, and berry composition of Ruby Seedless grapevines. American Journal of Enology and Viticulture 46 (4):559-563

Ferreira V, Lopez R (2019) The Actual and Potential Aroma of Winemaking Grapes. Biomolecules 9 (12):818

Flamini R, De Rosso M, Panighel A, Dalla Vedova A, De Marchi F, Bavaresco L (2014) Profiling of grape monoterpene glycosides (aroma precursors) by ultra-high performanceliquid chromatography-high resolution mass spectrometry (UHPLC/QTOF). J Mass Spectrom 49 (12):1214-1222. doi:10.1002/jms.3441

Fung RW, Gonzalo M, Fekete C, Kovacs LG, He Y, Marsh E, McIntyre LM, Schachtman DP, Qiu W (2008) Powdery mildew induces defense-oriented reprogramming of the transcriptome in a susceptible but not in a resistant grapevine. Plant physiology 146 (1):236-249

Galletto L, Barisan L, Boatto V, AC Costantini E, Lorenzetti R, Pomarici E, Vecchio R (2014) More crop for drop-climate change and wine: an economic evaluation of a new droughtresistant rootstock. Recent patents on food, nutrition \& agriculture 6 (2):100-112

Ghaste M, Narduzzi L, Carlin S, Vrhovsek U, Shulaev V, Mattivi F (2015) Chemical composition of volatile aroma metabolites and their glycosylated precursors that can uniquely differentiate individual grape cultivars. Food Chem 188:309-319. doi:10.1016/j.foodchem.2015.04.056

Gika HG, Theodoridis GA, Plumb RS, Wilson ID (2014) Current practice of liquid chromatography-mass spectrometry in metabolomics and metabonomics. J Pharm Biomed Anal 87:12-25. doi:10.1016/j.jpba.2013.06.032

Gouthu S, Deluc LG (2015) Timing of ripening initiation in grape berries and its relationship to seed content and pericarp auxin levels. BMC plant biology 15 (1):46 
Gowda H, Ivanisevic J, Johnson CH, Kurczy ME, Benton HP, Rinehart D, Nguyen T, Ray J, Kuehl J, Arevalo B, Westenskow PD, Wang J, Arkin AP, Deutschbauer AM, Patti GJ, Siuzdak G (2014) Interactive XCMS Online: Simplifying Advanced Metabolomic Data Processing and Subsequent Statistical Analyses. Analytical Chemistry 86 (14):69316939. doi:10.1021/ac500734c

Gross JH (2006) Mass spectrometry: a textbook. Springer Science \& Business Media,

Gu S, Read PE, Gamet S (2005) Performance of 'Gewurztraminer'on six rootstocks under marginal climatic conditions. Grapevine Rootstocks: Current Use, Research, and Application:57

Guth H (1997) Quantitation and Sensory Studies of Character Impact Odorants of Different White Wine Varieties. Journal of Agricultural and Food Chemistry 45 (8):3027-3032. doi:10.1021/jf970280a

Hampel D, Robinson AL, Johnson A, Ebeler SE (2014) Direct hydrolysis and analysis of glycosidically bound aroma compounds in grapes and wines: comparison of hydrolysis conditions and sample preparation methods. Australian journal of grape and wine research 20 (3):361-377

Harbertson JF, Keller M (2011) Rootstock Effects on Deficit-Irrigated Winegrapes in a Dry Climate: Grape and Wine Composition. American Journal of Enology and Viticulture 63 (1):40-48. doi:10.5344/ajev.2011.11079

Hjelmeland AK, Ebeler SE (2014) Glycosidically Bound Volatile Aroma Compounds in Grapes and Wine: A Review. American Journal of Enology and Viticulture 66 (1):1-11. doi:10.5344/ajev.2014.14104

Hogan S, Zhang L, Li J, Zoecklein B, Zhou K (2009) Antioxidant properties and bioactive components of Norton (Vitis aestivalis) and Cabernet Franc (Vitis vinifera) wine grapes. LWT-Food Science and Technology 42 (7):1269-1274 
Holopainen JK, Blande JD (2012) Molecular plant volatile communication. In: Sensing in nature. Springer, pp 17-31

Ilc T, Werck-Reichhart D, Navrot N (2016) Meta-analysis of the core aroma components of grape and wine aroma. Frontiers in Plant Science 7:1472

Jackson RS (2008) Wine science: principles and applications. Academic press,

James G, Witten D, Hastie T, Tibshirani R (2013) An introduction to statistical learning, vol 112. Springer,

Jogaiah S, Striegler KR, Bergmeier E, Harris J (2012) Influence of cluster exposure to Sun on fruit composition of 'Norton' grapes (vitis estivalis michx) in Missouri. International journal of fruit science $12(4): 410-426$

Jogaiah S, Striegler KR, Bergmeier E, Harris J (2013) Influence of canopy management practices on canopy characteristics, yield, and fruit composition of 'Norton'grapes (Vitis aestivalis Michx). International journal of fruit science 13 (4):441-458

JONES TH, CULLIS BR, CLINGELEFFER PR, RÜHL EH (2009) Effects of novel hybrid and traditional rootstocks on vigour and yield components of Shiraz grapevines. Australian Journal of Grape and Wine Research 15 (3):284-292. doi:10.1111/j.17550238.2009.00061.x

Katajamaa M, Miettinen J, Orešič M (2006) MZmine: toolbox for processing and visualization of mass spectrometry based molecular profile data. Bioinformatics 22 (5):634-636

Keller M, Mills LJ, Harbertson JF (2012) Rootstock effects on deficit-irrigated winegrapes in a dry climate: Vigor, yield formation, and fruit ripening. American Journal of Enology and Viticulture 63 (1):29-39

Kennedy J (2002) Understanding grape berry development. Practical winery \& vineyard 4:1-5 Kliewer W (1965) Changes in Concentration of Glucose, Fructose, and Total Soluble Solids in Flowers and Berries of Vitis Vlnifera. American Journal of Enology and Viticulture 16 (2):101-110 
Kliewer WM, Howarth L, Omori M (1967) Concentrations of tartaric acid and malic acids and their salts in Vitis vinifera grapes. American Journal of Enology and Viticulture 18 (1):42-54

Koundouras S, Hatzidimitriou E, Karamolegkou M, Dimopoulou E, Kallithraka S, Tsialtas JT, Zioziou E, Nikolaou N, Kotseridis Y (2009) Irrigation and rootstock effects on the phenolic concentration and aroma potential of Vitis vinifera L. cv. cabernet sauvignon grapes. J Agric Food Chem 57 (17):7805-7813. doi:10.1021/jf901063a

Liland KH (2011) Multivariate methods in metabolomics - from pre-processing to dimension reduction and statistical analysis. TrAC Trends in Analytical Chemistry 30 (6):827-841. doi:https://doi.org/10.1016/j.trac.2011.02.007

Liu HF, Wu BH, Fan PG, Li SH, Li LS (2006) Sugar and acid concentrations in 98 grape cultivars analyzed by principal component analysis. Journal of the Science of Food and Agriculture 86 (10):1526-1536

Main G (2005) Growing and vinting Cynthiana. Norton grapes. Proceedings of the 24th Annual Horticulture Industries Show ...,

Main G, Morris J, Striegler K (2002) Rootstock effects on Chardonel productivity, fruit, and wine composition. American Journal of Enology and Viticulture 53 (1):37-40

Mansfield AK, Schirle-Keller J-P, Reineccius GA (2011) Identification of odor-impact compounds in red table wines produced from Frontenac grapes. American journal of enology and viticulture:ajev. 2011.10067

Mendes-Pinto MM (2009) Carotenoid breakdown products the — norisoprenoids — in wine aroma. Archives of Biochemistry and Biophysics 483 (2):236-245. doi:https://doi.org/10.1016/j.abb.2009.01.008

Migicovsky Z, Harris ZN, Klein LL, Li M, McDermaid A, Chitwood DH, Fennell A, Kovacs LG, Kwasniewski M, Londo JP, Ma Q, Miller AJ (2019) Rootstock effects on scion 
phenotypes in a 'Chambourcin' experimental vineyard. Horticulture Research 6 (1):64. doi:10.1038/s41438-019-0146-2

Mirás-Avalos JM, Intrigliolo DS (2017) Grape Composition under Abiotic Constrains: Water Stress and Salinity. Frontiers in Plant Science 8 (851). doi:10.3389/fpls.2017.00851

Mudge K, Janick J, Scofield S, Goldschmidt EE (2009) 9 A History of Grafting. Horticultural reviews 35:437

Nelson CC, Kennedy JA, Zhang Y, Kurtural SK (2015) Applied Water and Rootstock Affect Productivity and Anthocyanin Composition of Zinfandel in Central California. American Journal of Enology and Viticulture 67 (1):18-28. doi:10.5344/ajev.2015.15043

Noble AC, Ebeler SE (2002) Use of multivariate statistics in understanding wine flavor. Food Reviews International 18 (1):1-20. doi:10.1081/fri-120003414

Otto M (2016) Chemometrics: statistics and computer application in analytical chemistry. John Wiley \& Sons,

Parker M, Capone DL, Francis IL, Herderich MJ (2017) Aroma Precursors in Grapes and Wine: Flavor Release during Wine Production and Consumption. J Agric Food Chem. doi:10.1021/acs.jafc.6b05255

Polaskova P, Herszage J, Ebeler SE (2008) Wine flavor: chemistry in a glass. Chem Soc Rev 37 (11):2478-2489. doi:10.1039/b714455p

Poole C (2010) Chromatography: Concepts and Contrasts. Springer,

Qian MC, Fang Y, Shellie K (2009) Volatile Composition of Merlot Wine from Different Vine Water Status. Journal of Agricultural and Food Chemistry 57 (16):7459-7463. doi:10.1021/jf9009558

Rambla JL, Trapero-Mozos A, Diretto G, Rubio-Moraga A, Granell A, Gomez-Gomez L, Ahrazem O (2016) Gene-Metabolite Networks of Volatile Metabolism in Airen and Tempranillo Grape Cultivars Revealed a Distinct Mechanism of Aroma Bouquet Production. Front Plant Sci 7:1619. doi:10.3389/fpls.2016.01619 
Rapp A, Mandery H (1986) Wine aroma. Experientia 42 (8):873-884

Reisch BI, Goodman RN, Martens M-H, Weeden NF (1993) The Relationship Between Norton and Cynthiana, Red. Wine Cultivars Derived from Vitis aestivalis. American journal of enology and viticulture $44(4): 441-444$

Reynolds AG, Wardle DA (2001) Rootstocks impact vine performance and fruit composition of grapes in British Columbia. HortTechnology 11 (3):419-427

Rice S, Tursumbayeva M, Clark M, Greenlee D, Dharmadhikari M, Fennell A, Koziel JA (2019) Effects of harvest time on the aroma of white wines made from cold-hardy Brianna and Frontenac gris grapes using headspace solid-phase microextraction and gas chromatography-mass spectrometry-olfactometry. Foods 8 (1):29

Ristic R, Iland PG (2005) Relationships between seed and berry development of Vitis vinifera L. cv Shiraz: developmental changes in seed morphology and phenolic composition. Australian Journal of grape and wine research 11 (1):43-58

Robinson AL, Boss PK, Solomon PS, Trengove RD, Heymann H, Ebeler SE (2014) Origins of grape and wine aroma. Part 1. Chemical components and viticultural impacts. American Journal of Enology and Viticulture 65 (1):1-24

Robinson AL, Ebeler SE, Heymann H, Boss PK, Solomon PS, Trengove RD (2009) Interactions between wine volatile compounds and grape and wine matrix components influence aroma compound headspace partitioning. J Agric Food Chem 57 (21):10313-10322. doi:10.1021/jf902586n

Ruffner H (1982a) Metabolism of tartaric and malic acids in Vitis V a review. A Vitis-,: 247V259

Ruffner H (1982b) Metabolism of tartaric and malic acids in Vitis: A review-Part B. Vitis 21 (247-259):65

Sabbatini P, Howell GS (2013) Rootstock scion interaction and effects on vine vigor, phenology, and cold hardiness of interspecific hybrid grape cultivars (Vitis spp.). International journal of fruit science $13(4): 466-477$ 
Saito K, Matsuda F (2010) Metabolomics for functional genomics, systems biology, and biotechnology. Annu Rev Plant Biol 61:463-489.

doi:10.1146/annurev.arplant.043008.092035

Savoi S, Wong DCJ, Arapitsas P, Miculan M, Bucchetti B, Peterlunger E, Fait A, Mattivi F, Castellarin SD (2016) Transcriptome and metabolite profiling reveals that prolonged drought modulates the phenylpropanoid and terpenoid pathway in white grapes (Vitis vinifera L.). BMC Plant Biology 16 (1):67. doi:10.1186/s12870-016-0760-1

Schrimpe-Rutledge AC, Codreanu SG, Sherrod SD, McLean JA (2016) Untargeted Metabolomics Strategies — Challenges and Emerging Directions. Journal of The American Society for Mass Spectrometry 27 (12):1897-1905. doi:10.1007/s13361-016$1469-\mathrm{y}$

Schwab W, Wüst M (2015) Understanding the Constitutive and Induced Biosynthesis of Monoand Sesquiterpenes in Grapes (Vitis vinifera): A Key to Unlocking the Biochemical Secrets of Unique Grape Aroma Profiles. Journal of Agricultural and Food Chemistry 63 (49):10591-10603. doi:10.1021/acs.jafc.5b04398

Slegers A, Angers P, Ouellet E, Truchon T, Pedneault K (2015) Volatile Compounds from Grape Skin, Juice and Wine from Five Interspecific Hybrid Grape Cultivars Grown in Quebec (Canada) for Wine Production. Molecules 20 (6):10980-11016.

doi:10.3390/molecules200610980

Slegers A, Angers P, Pedneault K (2017) Volatile Compounds from Must and Wines from Five White Grape Varieties. Journal of Food Chemistry and Nanotechnology 03 (01). doi:10.17756/jfcn.2017-031

Stockert CM, Bisson LF, Adams DO, Smart DR (2013) Nitrogen status and fermentation dynamics for Merlot on two rootstocks. American Journal of Enology and Viticulture 64 (2):195-202 
Striegler R, Howell G (2015) The influence of rootstock on the cold-hardiness of Seyval grapevines 1. Primary and secondary effects on growth, canopy development, yield, fruit quality and cold hardiness. VITIS-Journal of Grapevine Research 30 (1):1

Striegler R, Howell G, Flore J (1993) Influence of rootstock on the response of Seyval grapevines to flooding stress. American journal of enology and viticulture 44 (3):313-319

Sun Q, Gates MJ, Lavin EH, Acree TE, Sacks GL (2011) Comparison of odor-active compounds in grapes and wines from vitis vinifera and non-foxy American grape species. J Agric Food Chem 59 (19):10657-10664. doi:10.1021/jf2026204

Sweetman C, Deluc LG, Cramer GR, Ford CM, Soole KL (2009) Regulation of malate metabolism in grape berry and other developing fruits. Phytochemistry 70 (11-12):13291344

Tautenhahn R, Patti GJ, Rinehart D, Siuzdak G (2012) XCMS Online: a web-based platform to process untargeted metabolomic data. Analytical chemistry 84 (11):5035-5039

This P, Lacombe T, Thomas MR (2006) Historical origins and genetic diversity of wine grapes. Trends Genet 22 (9):511-519. doi:10.1016/j.tig.2006.07.008

Thomas AL, Harris JL, Bergmeier EA, Striegler RK (2017) Establishment Technique and Rootstock Impact ‘Chambourcin'Grapevine Morphology and Production in Missouri. HortTechnology 27 (2):248-256

Tramontini S, Vitali M, Centioni L, Schubert A, Lovisolo C (2013) Rootstock control of scion response to water stress in grapevine. Environmental and experimental botany 93:20-26

Want E, Masson P (2011) Processing and analysis of GC/LC-MS-based metabolomics data. In: Metabolic profiling. Springer, pp 277-298

Winterhalter P, Rouseff R (2001) Carotenoid-Derived Aroma Compounds: An Introduction. In: Carotenoid-Derived Aroma Compounds, vol 802. ACS Symposium Series, vol 802. American Chemical Society, pp 1-17. doi:doi:10.1021/bk-2002-0802.ch001 
10.1021/bk-2002-0802.ch001

Wooldridge J, Louw P, Conradie W (2010) Effects of rootstock on grapevine performance, petiole and must composition, and overall wine score of Vitis vinifera cv. Chardonnay and Pinot Noir. South African Journal of Enology and Viticulture 31 (1):45-48

Xia J, Wishart DS (2016) Using MetaboAnalyst 3.0 for Comprehensive Metabolomics Data Analysis. Current Protocols in Bioinformatics 55 (1):14.10.11-14.10.91. doi:10.1002/cpbi.11

Xu C-H, Chen G-S, Xiong Z-H, Fan Y-X, Wang X-C, Liu Y (2016) Applications of solid-phase microextraction in food analysis. TrAC Trends in Analytical Chemistry 80:12-29. doi:https://doi.org/10.1016/j.trac.2016.02.022

Zhang E, Chai F, Zhang H, Li S, Liang Z, Fan P (2017) Effects of sunlight exclusion on the profiles of monoterpene biosynthesis and accumulation in grape exocarp and mesocarp. Food chemistry 237:379-389 


\title{
Chapter 2: Identification of aroma markers for Norton and Cabernet Sauvignon berries and wines to characterize the $\mathrm{F} 1$ population.
}

\begin{abstract}
:
Due to their disease tolerance and cold hardy nature, interspecific hybrid grapes are widely grown in the Midwestern United States, including Missouri. However, the aroma profile of these hybrids is unique and generally less popular in comparison to the V. vinifera grapes. We investigated the volatile precursors and free volatiles in Norton grapes, a popular hybrid in Missouri, using the more inclusive non-targeted metabolomics approach. We analyzed 21 Norton and 21 Cabernet Sauvignon (Cab) grapes from different sites and vintages for the free and bound volatile compounds. Additionally, 10 commercial Norton and Cab wines were also analyzed for their volatile profiles, which were detected using HS-SPME-GCMS. The data generated from GCMS were analyzed by XCMS software for differences between the two cultivars. The two cultivars were found to have differences in their volatile profiles, with 825 features different for free grape volatiles, 826 features different for bound volatiles, and 403 features different for wine volatiles at 0.05 significance level and with at least a 1.5 -fold change between the two cultivars. Those features were used to identify several odor active compounds in both grapes and wines, including $\beta$-damascenone, $\beta$-Ionone, Eugenol, TDN, Ethyl hexadecanoate, and Methyl salicylate. Some of the identified compounds were higher in Norton than Cab, however, several features were higher in Cab. Using the identified aroma compounds as markers, we phenotyped the F1 population of Norton and Cab. The F1 population was found to be segregating for many aroma compounds with some genotypes demonstrating an even higher concentration of aroma volatiles than either of the parents. Ultimately, using this phenotype data,
\end{abstract}


quantitative trait loci (QTL) mapping will be performed to identify regions of the genome directly impacting these traits which may prove useful in future work related to developing varieties with the viticultural benefits of Norton but an aroma profile closer to Cab. 


\section{Introduction:}

The ultimate aim in all hybrid grape breeding programs is to develop varieties with strong resistance to biotic and abiotic stresses but with flavor attributes that are at least as good as the cultivars that are currently preferred by consumers. Vitis vinifera is the most widely cultivated grapevine species throughout the world among 60 species belonging to the genus Vitis (Narduzzi et al. 2015). In the great diversity of Vitis, there are species adapted for drought, extreme cold, pest-infested soils, fungal infections, and more, however, they suffer both in fruit quality and by not being self-fertile (Reisch et al. 2012; Jackson 2008). Vitis vinifera, however, has been used for millennia to produce high-quality wines but lacks the physiological traits to thrive in many of the areas where wine is currently produced. Interspecific hybrids are developed by crossing Vitis vinifera, for its superior fruit quality with other the stress-resistant Vitis species (Reisch et al. 2012). While hybrid wines play an important role in some wine markets, they ultimately lag behind vinifera in terms of consumer acceptability due to the inheritance of negative aromas from their North American native parents (Biasoto et al. 2014). Initial research focused on finding "impact odorants" or single compounds that could be blamed for hybrids having less preferred wine quality. While some compounds such as methyl anthranilate and o-aminoacetophenone were identified in Vitis labrusca and its progeny (Shure and Acree 1994), recent work suggests that the issue with developing wine grapes of high quality is more complex then omitting a handful of aroma compounds. To properly express all-important flavor differences between a consumer-preferred vinifera wine and a less preferred interspecific hybrid wine, it may be better to cast a bigger net and use a metabolomics-based approach, where potentially important information or markers are not omitted in the interest of a simple answer. Ultimately, this may allow 
identification of complex trends and variability among populations that would have been overlooked using a targeted analysis for a limited number of aroma active compounds.

Much of the diversity of wine aroma is derived from the grape and as such a great deal of research has been conducted on grape and wine aroma chemistry (Ebeler and Thorngate 2009). Volatile aroma compounds and their precursors derived from grapes give a distinct varietal character to the wine produced from different cultivars (Mateo and Jiménez 2000). The varietal characteristics can sometimes be attributed to a single or few distinct aroma compounds, in others, they are attributed to the interaction of many aroma compounds and the presence of matrix effects (Hjelmeland and Ebeler 2014; Villamor and Ross 2013; Ferreira et al. 2000). Grape derived aromas can be classified as free volatiles that is found in grapes and bound volatiles, which are compounds that are glycosidically bound, or bound to amino acids and can be released to produce a volatile aroma active compound during vinification (Ebeler 2001; Ebeler and Thorngate 2009). Bound volatiles is potential aroma contributors that are basically aglycones linked to the sugar moiety (Ebeler and Thorngate 2009). Aglycones constitute mostly terpenes, C13norisoprenoids, volatile phenols, aromatic phenols, C6 compounds, aliphatic alcohols, aliphatic acids, benzenic, and phenolic acid derivatives (Ebeler 2001). While both free and bound volatiles are present in skin and pulp, the skin has the highest concentration of aroma compounds and mostly used in the volatile analysis of grapes (Callejon et al. 2012; Canuti et al. 2009; Slegers et al. 2015). The difference in the proportion of the glycosidic aroma compounds and the type of aglycone associated with the bound volatiles tend to differ between cultivars that render the varietal aroma distinction between varieties (Hjelmeland and Ebeler 2014; Hjelmeland et al. 2015; Ebeler 2001; Ebeler and 
Thorngate 2009; Hampel et al. 2014; Polaskova et al. 2008; Robinson et al. 2009). Nonvolatile aroma precursors are not only the reservoirs of desirable wine aroma and flavor attributes but may also give rise to some of the undesirable wine aromas and flavors including smoke taint, reductive characters, and geranium taint in wine (Parker et al. 2017). Therefore, it is very important to understand the volatile as well as nonvolatile aroma precursors present in grapes that can be potential aroma odorant in the wine. Enzyme hydrolysis has been preferably used to study the aroma precursors in grapes as it can efficiently release aglycones from the glucosides in contrast to acid hydrolysis as the formation of aroma artifacts has been found to be lesser in comparison to acid hydrolysis (Gunata et al. 1994; Hampel et al. 2014). Therefore, glycosidase enzyme hydrolysis was used to release aglycones from the glycosides before being analyzed by GC-MS.

When developing hybrid cultivars, the undesirable aroma characteristics of the native species tend to be inherited together with desired traits, which makes the wines from the hybrids less preferable to the consumers. The unique chemistry of hybrid wines is a challenge for wine-makers to produce consumer-preferred wine and often great efforts are made to make the wines more "vinifera-like" (Narduzzi et al. 2015; Sun et al. 2011b; de Pinho and Bertrand 1995). The hybrid grapes have been found to contain several undesirable volatile aroma compounds as well as a higher concentration of nonfruity aroma compounds than vinifera cultivars (Chisholm et al. 1994; Narduzzi et al. 2015; Slegers et al. 2015; Skinkis et al. 2008). Vitis labrusca varieties and hybrids derived from labrusca are associated with the characteristic "foxy" flavor due to the presence of methyl anthranilate and o-aminoacetophenone (Reynolds et al. 1982; Nelson et al. 1977; Sun et al. 2011a; Kolor 1983; Moyer and Mattick 1976). The non-labrusca 
North American species do not possess the foxy aroma of the labrusca varieties and no specific compound has been identified (Slegers et al. 2015; Sun et al. 2011a). Although few targeted studies to understand the volatile composition of grapes, must and wines have been done in hybrid cultivars and native species, a detailed comprehensive analysis of the free and bound volatiles in hybrid grapes and wines and their comparison with the vinifera grapes and wines have been lacking (Nisbet et al. 2014; Slegers et al. 2015; Slegers et al. 2017; Sun et al. 2011a). Among a few papers that looked at the volatile compounds in interspecific hybrids using a targeted approach, no compound was found that was different from each other. With a targeted approach, a few significant compounds are only targeted and thus quantified, however with a non-targeted approach, the new possibilities of finding the new, unknown compound are possible, as it aims for comprehensive analysis of all volatiles present in the sample (Commisso et al. 2013; Narduzzi et al. 2015; Roullier-Gall et al. 2014b; Schrimpe-Rutledge et al. 2016).

With the expansion of grape-growing in the Mid-Western United States, the production of traditional $V$. vinifera cultivars, which do not cope well with the extreme cold, humidity, and rains and is susceptible to a variety of fungal diseases. The principal grapes cultivated in Missouri are native American, French-American hybrids, and hybrids that can survive well in the hot, humid summers and cold winters. Norton is a widely recognized and grown hybrid grape in Missouri, derived from $V$. aestivalis and $V$. vinifera (Ambers 2013). Norton is favorable for humid regions with long growing seasons and has demonstrated good resistance to many fungal diseases like powdery mildew, downy mildew, Botrytis bunch rot, and black rot, as well as Pierce's disease and Phylloxera (Reisch et al. 1993; Sapkota et al. 2015). Although Norton does not possess 
the characteristic foxy odor of many hybrid grapes, the high malic acid and phenolics concentration in the Norton grapes possess many problems in winemaking that negatively impact wine quality. It also has a very distinctive aroma that clearly differentiates it from V. vinifera.

Cabernet Sauvignon is a widely cultivated Vitis vinifera grape cultivar that is widely accepted for the high-quality red wine. However, it is not cold tolerant and is susceptible to many diseases. Several grape breeders have made crosses between Norton and Cabernet Sauvignon with the hope of producing a variety with the wine quality of Cabernet Sauvignon and the environmental and biological tolerances of Norton. This is despite not having much information on what causes the flavor differences between the varieties (e.g. are the differences due to key impact odorants or just variations in the concentration of common compounds). The flavor is among the key factors of grape and wine quality but despite the analytical and biochemical knowledge about volatiles, little is known about the genetic basis of aroma (Doligez et al. 2006). Therefore, this project aims to identify the free and bound aroma compounds that exhibit differences in Norton and Cabernet Sauvignon grapes and wines. The other objective of the study is to confirm and quantify those compounds. This is immediately useful, as knowing what compounds are aromatically important it is possible to leverage previous research to adjust viticultural and winemaking practices to improve quality. The ultimate goal of the study is to use known key volatile differences between Norton and Cabernet Sauvignon to identify distinct aromatic phenotypes within Missouri State University’s Norton x Cabernet Sauvignon population that can be leveraged in finding markers for expedited breeding. While a lot of studies have been conducted on aroma compounds using 
analytical and biochemical focus, little is known about the genetic and molecular basis of aroma compounds (Doligez et al. 2006; Battilana et al. 2009; Guillaumie et al. 2013). With the development of reference grape genomes and the ability to genotype plants, genetic maps of grapevines have been readily accessible (Sapkota et al. 2019) and the development of high-resolution mass spectrometry has made possible phenotyping of compounds that are present in low concentrations. This availability of genetic maps and the ability to measure aroma volatiles have provided the opportunity to study the genetics of regulation of the complex aroma phenotypes in plants.

\section{Materials and Methods:}

\subsection{Chemicals:}

All aroma standards other than 1,1,6-trimethyl-1,2-dihydronapthalene (TDN) were purchased from Sigma-Aldrich (St. Louis, MO, USA) at >98.8\%. TDN was synthesized and donated from Dr. Gavin Sacks lab at Cornell University (Kwasniewski et al. 2010). A C7-C30 hydrocarbon mixture, used for determination of Kovats retention indices was obtained from Sigma-Aldrich. Sodium chloride was purchased from Fisher Chemicals (Fair Lawn, NJ, USA). Ultrapure water (Type 1 water) was prepared using the ELGA Lab Water PURELAB Classic (High Wycombe, UK). L-Tartaric acid (99\%) was obtained from Sigma-Aldrich.

\subsection{Fruit samples:}

The Norton and Cabernet Sauvignon grapes used in this experiment were harvested in 2012 and 2013 from Rocheport, $\mathrm{MO}$ (lat:38 , 58'N, long: 92 ${ }^{\circ} 33^{\prime} \mathrm{W}$, elevation: 240m), Mountain Grove, MO, and Virginia. All vines were high wire cordon 
trained and own-rooted. Representative triplicate samples in 2012 and 2013 were taken at veraison, pre-harvest, harvest, and post-harvest. The samples were frozen after harvesting.

\subsection{Sample preparation for free volatiles:}

For the extraction of free volatiles from the grape berries, the grape berries were harvested and stored at $-80^{\circ} \mathrm{C}$ until used for the analysis. For analysis, the 60 grams of the grape berries were thawed and then ground using a handheld grinder. To prevent oxidation of the berries, $50 \mu \mathrm{l}$ ascorbic acid (200g/L stock concentration) was added before grinding. The ground grapes were centrifuged at $11000 \mathrm{rpm}$ using Eppendorf 5840R for 15 minutes at $4^{\circ} \mathrm{C}$. The supernatant was discarded and the grape solids (mostly skins) were further extracted overnight with sodium phosphate extraction buffer $(0.1 \mathrm{M}$ sodium phosphate extraction buffer of $13 \%$ ethanol by volume adjusted to a $\mathrm{pH}$ of 4.5 ) in a circular shaker (Labnet Orbit 1000) at $105 \mathrm{rpm}$ for 16 hours overnight, and then centrifuged for 15 minutes at $11000 \mathrm{rpm}$ (model 5804 R, Eppendorf AG, Hamburg) to separate the solids from the extract. The supernatant was pipetted off and used for further analysis.

In a $15 \mathrm{ml}$ amber SPME glass vial, $5 \mathrm{ml}$ of the extract was taken and $50 \mu \mathrm{l}$ of internal standards were added to make a final concentration of $0.1 \mathrm{mg} / \mathrm{L}(10 \mathrm{ppb}) 2$ Octanol and $1 \mathrm{mg} / \mathrm{L}(100 \mathrm{ppb})$ of 4-methyl-2-pentanol, followed by the addition of $2 \mathrm{gm}$ $\mathrm{NaCl}$ per vial. The samples were then processed for volatile aroma compounds using the HS-SPME-GC-MS. 


\subsection{Sample preparation for total/bound volatiles:}

Bound volatiles was extracted using a method adapted from Hampel et al. (2014). 60 grams of frozen grapes samples were thawed and ground using a handheld grinder after the addition of $50 \mu \mathrm{l}$ ascorbic acid (200g/L stock concentration) to prevent oxidation of the grapes. The ground grapes were centrifuged at $11000 \mathrm{rpm}$ for 15 minutes at $4^{\circ} \mathrm{C}$ (model 5804 R, Eppendorf AG, Hamburg) to separate the juice from grape solids. The supernatant was discarded and the skins were extracted for the bound volatile compounds by adding $30 \mathrm{ul}$ of $0.1 \mathrm{M}$ sodium phosphate buffer of $13 \%$ ethanol by volume adjusted to a pH of 4.5 in a circular shaker (Labnet Orbit 1000) at $105 \mathrm{rpm}$ for 16 hours overnight and then centrifuged for 15 minutes at $11000 \mathrm{rpm}$ (model $5804 \mathrm{R}$, Eppendorf AG, Hamburg) to separate the solids from the extract. The supernatant was pipetted off and used for further analysis.

A stock Rapidase enzyme solution (250,000 mg/L) (lot no 91542020P, DSM)) was prepared using $2.5 \mathrm{~g}$ of Rapidase in $10 \mathrm{ml}$ of DI water. In a $15 \mathrm{ml}$ glass amber vial, 5 $\mathrm{ml}$ aliquots of the supernatant were spiked with $20 \mu 1$ of the Rapidase enzyme solution to yield a final enzyme concentration of $1000 \mathrm{ppm}, 50 \mu \mathrm{l}$ of the internal standard solution to yield a final concentration of $0.1 \mathrm{mg} / \mathrm{L}(10 \mathrm{ppb}) 2-\mathrm{Octanol}$ and $1 \mathrm{mg} / \mathrm{L}(100 \mathrm{ppb})$ of 4methyl-2-pentanol, and $10 \mu \mathrm{l}$ of glycosidic standards to yield final glycoside concentrations of 2000 ppb Phenyl- $\beta$-D-glucopyranoside and 100 ppb of octyl $\beta$-Dglucopyranoside. The glass vials were sealed and incubated in a $45^{\circ} \mathrm{C}$ water bath for four hours. The vials were then cooled to ambient temperature in a $25^{\circ} \mathrm{C}$ water bath for 10 minutes to prevent headspace volatilization. $2 \mathrm{~g}$ of $\mathrm{NaCl}$ was added to the sample vial to inactivate the Rapidase enzyme. The samples were then processed for volatile aroma 
compounds using the HS-SPME-GC-MS method. The glycosidic standards were added to know the conversion ratio of the enzyme.

\subsection{Sample preparation for wine:}

A total of 22 commercial wines with 11 wines of Norton and Cabernet Sauvignon each from different states (Missouri and California) and countries (the USA and Australia) were used in this study. To prevent oxidation of the wine, the wine bottles were opened in a vacuum chamber to create an inert condition. In a $15 \mathrm{ml}$ amber glass vial, $5 \mathrm{ml}$ wines were spiked with $50 \mu 1$ of the internal standard solution to yield a final internal concentration of $0.5 \mathrm{mg} / \mathrm{L}$ (50 ppb) 2-Octanol. To the sample vials, $2 \mathrm{~g}$ of NaCl was added to inactivate the enzymes and improve headspace partitioning (Canuti et al. 2009). The vials were then sealed and analyzed using the HS SPME GC-MS method outlined below.

\subsection{Headspace solid-phase microextraction gas chromatography mass spectrometry (GC-MS) analysis:}

The SPME fiber used for sampling and extraction of the odor-active compounds from the headspace of the samples was a $65 \mu \mathrm{m}$ polydimethylsiloxane (PDMS)/divinylbenzene (DVB) 1cm SPME fiber, 23 gauge (Supelco). Fibers were conditioned before use according to the manufacturer's recommendations. The samples (grape extracts and wines), in a $15 \mathrm{ml}$ amber glass vials, were preincubated for 15 minutes at $45^{\circ} \mathrm{C}$ to ensure consistent temperature during extraction. The fiber was exposed for $45 \mathrm{~min}$ at $45^{\circ} \mathrm{C}$ in the headspace above the sample prior to GC-MS analysis. All samples were agitated at $500 \mathrm{rpm}$ during extraction. 
The HS-SPME GC-MS system consisted of a PAL autosampler (Varian) mounted on an Agilent 7890B gas chromatograph (Santa Clara, CA, USA) coupled with an Agilent 5977A mass selective detector (MSD). The $65 \mu \mathrm{m}$ PDMS/DVB SPME fiber was desorbed in the inlet at $250{ }^{\circ} \mathrm{C}$ for $2 \mathrm{~min}$ in spitless mode (inlet glass liner/SPME direct, 0.75mm I.D, Supelco), after which the spit flow was turned on $(50 \mathrm{~mL} / \mathrm{min})$ for the remainder of the GC-MS run; the SPME fiber was conditioned in the inlet for $14.7 \mathrm{~min}$ before it was inserted into the next sample. No carry-over was observed between samples. A DB-WaxETR column (30m x 0.25mm I.D, $0.25 \mu \mathrm{m}$ film thickness; Agilent, Santa Clara, CA, USA) and helium carrier gas at a flowrate of $1.2 \mathrm{~mL} / \mathrm{min}$ was used for all analyses. The GC oven temperature program was as follows: the initial temperature was $40{ }^{\circ} \mathrm{C}$ for 1.0 min then was increased to $200^{\circ} \mathrm{C}$ at $5^{\circ} \mathrm{C} / \mathrm{min}$ followed by a second increase at $12{ }^{\circ} \mathrm{C} / \mathrm{min}$ to the final temperature of $240^{\circ} \mathrm{C}$, which was held for $10 \mathrm{~min}$. the mass selective detector was operated in SIM/Scan mode, including SIM parameters for allowing adequate $\beta$-damascenone detection limits $(121 \mathrm{~m} / \mathrm{z} ; 190 \mathrm{~m} / \mathrm{z}$, range $40-250 \mathrm{~m} / \mathrm{z}$; $6.4 \mathrm{scans} / \mathrm{s}$ ), and the MS transfer line was maintained at $240{ }^{\circ} \mathrm{C}$.

\subsection{Data processing using untargeted metabolomics analysis:}

The raw data acquired by Masslynx from GC-MS were converted to.CDF (common data format) data using the OpenChrome software. Then, the .cdf files were loaded to XCMS online software as a paired batch job, where the files for Norton and Cabernet Sauvignon were uploaded and submitted for analysis. XCMS constructs the mass-RT and mass intensity matrix, aligned across all the samples. Automatic feature detection was then performed on all data sets with the XCMS online software (https://xcmsonline.scripps.edu/landing_page.php?pgcontent=mainPage) using the 
default parameters (Feature detection: centWave method, min. and max. peak width=5 and $20, \mathrm{~S} / \mathrm{N}$ thresholds $=6$, mzdiff $=0.01$, integration method $=1$, prefilter peaks $=3$, prefilter intensity $=100$, Noise filter $=0$, Retention time correction: Obiwarp method, profStep=1; Alignment: $\mathrm{mzwid}=0.015, \operatorname{minfrac}=0.5, \mathrm{bw}=5, \max =100, \operatorname{minsamp}=1)$. Once the job was done, the results were downloaded from the XCMS online, which were basically features having a definite $\mathrm{m} / \mathrm{z}$ ratio, retention time, and intensity. The features that are significant at 0.05 level and have higher or equal to 2 times fold change were filtered and used for further analysis. XCMS is a cloud-based platform that provides untargeted metabolomics workflow including feature detection, retention time correction, chromatogram alignment, metabolite feature detection, statistical data analysis, and putative identification (Gowda et al. 2014; Tautenhahn et al. 2012). Principal component analysis (PCA) was performed in log-transformed and autoscaled data. Partial least squares discriminant analysis (PLS-DA) were evaluated for sample discrimination.

\subsection{Identification of compound and confirmation:}

After identification of the significant features, the compounds represented by the features were identified based on the retention time using the NIST database.

Identification was achieved by comparing mass spectra obtained from the sample with those from the pure standards injected in the same conditions and by comparing the Kovats retention index and the mass spectra in the NIST 2.0 library database. The compounds identified were then further confirmed using the standard curve and retention time index. The important compounds or volatile compounds were then quantified using standard curve. The internal standard quantification method was used. 2-Octanol and 4Methyl-2-Pentanol were chosen as the internal standard. Quantitative data of the 
identified compounds were obtained by interpolation of the relative areas versus the internal standard area, in calibration graphs built for pure reference compounds.

\subsection{Screening F1 population using identified volatiles:}

The cross between Norton and Cabernet Sauvignon was made in 2005 and was planted in a Missouri State Fruit Experiment Station vineyard in 2007. Since grapevines are heterozygous, the F1 population is segregating and thus can be used as a mapping population to study the genetics of the traits. In the fall of 2016, the berries from $90 \mathrm{~F} 1$ genotypes (2 replication each) were harvested and then processed for the basic chemical analysis of the berries. The organic acids, malic, tartaric, and citric acid analysis were performed using the UPLC system using UV absorbance at $210 \mathrm{~nm}$. Free and bound compounds were also quantified in the F1 population using GC-MS/MS using the method described in section 2.6.

\subsection{Data analysis:}

The data were analyzed using MetaboAnalyst 3.0 (Xia and Wishart 2016) and R statistical software. In MetaboAnalyst, the features with more than 50\% missing values were removed, and the remaining missing values were replaced by a small value (half of the minimum positive value in the original data). Data filtering using interquartile range was then performed to remove features arising from the baseline noises and to remove features that do not change throughout the treatments that are unlikely to be used for further analysis. Then sample normalization was performed to account for the differences among the sample, for the wine data, normalization was done using a reference sample which is the 2-Octanol internal standard area. Then, the data was log-transformed and 
auto-scaled (mean-centered and divided by the standard deviation of each variable) and then used for further statistical analysis. The autoscaling method was used to remove any variation comprised during analysis (such as a loss of instrumental sensitivity) of an original HRMS peaks list.

Univariate statistics such as a t-test was performed for exploratory data analysis to identify the potentially significant features to discriminate the treatments under study. To consider the type II error with repeated tests, FDR correction was applied, and q-values were considered.

The principal component analysis was performed with significant features, which is a type of unsupervised method. PCA has the capacity to reduce the complexity of a huge dataset by extrapolating important information and displaying it as a set of new independent variables called principal components. Basically, it is important to know the similarity pattern of the observations or variables.

\section{Results:}

To investigate the differences in volatiles between Norton, an interspecific hybrid grapevine, and Cabernet Sauvignon, a vinifera grapevine, a comprehensive metabolomics-based approach was used for extraction of much information from the chromatograms and identification of compounds in an unbiased way. Although the berry samples analyzed were sourced from different locations, vintages and different states of ripening and wines analyzed were commercial wines from different locations and vintages, we identified more than thousand features (M(mass/charge) $\mathrm{T}$ (time in minutes)) that demonstrated differences between the two cultivars, and were then subjected to 
various multivariate analysis to find the compounds that were mainly driving differences between the two cultivars. The PCA scores plot indicated that there was a distinct separation in the volatile profile between the two cultivars (Fig 2.1). The significant feature differences (q value $<0.05$, fold change $>1.5$ ) were then used to tentatively identify 165 unique compounds with the NIST library, 34 of which were confirmed and quantified using authentic standards and calibration curves. Many compounds could not be identified from the features due to limitations of the database, potential co-elution causing combined spectra, and lack of authentic standards. The compounds that are quantified were again statistically analyzed and the results were in line with the untargeted feature extraction.

\subsection{Feature extraction:}

Using XCMS online, we identified 924, 793, and 1064 metabolic features $(\mathrm{M}(\mathrm{m} / \mathrm{z}) \mathrm{T}$ (retention time in minutes)) for free volatiles, total volatiles, and wine volatiles respectively that define differences between Norton and Cabernet Sauvignon (data not shown). The features that were significant at q-value $<0.05$ and had greater than or equal to 1.5 -fold change were selected and used for further statistical analysis and compound identification. We identified 418, 302 and 403 significant features that were significantly different ( $\mathrm{t}$-test, $\mathrm{q}$-value $<0.05)$ between Norton and Cabernet Sauvignon for free volatiles (Supplemental Table 1), total volatiles (Supplemental Table 2) and wine volatiles (Supplemental Table 3) respectively. All significant features, including their fold change and p-value, are given in the supplemental information (Table S1, S2, S3). Heatmaps made using the significant features also demonstrated that the two cultivars showed the opposite trend in feature intensities (Fig 2.1). 


\subsection{Free volatiles:}

To reduce the dimensionality of the data and better represent most of the variation of the data through few unrelated dimensions, unsupervised PCA was performed using the 418 significantly different features between Norton and Cabernet Sauvignon. Cabernet Sauvignon showed higher intensity of 240 metabolic features in comparison to Norton, whereas, Norton grapes have a higher intensity of 178 features (Table S1). The PCA scores plot demonstrated differences in the free volatile composition and proportion between two cultivars, in which PC1 explained $44.9 \%$ of the variation showing the genetic differences in aroma profile and PC2 explaining $14.1 \%$ of the variation mostly indicated variation between samples within the cultivar (Figure 2.2A). The two cultivars formed two separate groups without any overlap, indicates that the free volatiles in Norton and Cabernet Sauvignon might be very different. Additionally, we also performed PCA using development stages and location (figures not shown) and found no distinct separation based on either location or stages of development. This indicated that genetic differences were the most important driving factor to differentiate the free volatile profile in the two cultivars. Norton and Cabernet Sauvignon are derived from different species and thus that differences were distinctly manifested in the free volatile differences in grapes. It did not matter where the grapes come from or which development stages they were, they seemed to separate based on the genetic makeup of the cultivar. This demonstrated that Norton and Cabernet Sauvignon are very different grapes in terms of the free volatiles and thus this needs to be in consideration when making important decisions related to vineyard management as well as the winemaking decisions. 


\subsection{Total/Bound volatiles:}

In our study, the total volatiles represents the total of all volatiles present in the berries including free volatiles and volatiles released from precursors due to enzymatic action. The Norton and Cabernet Sauvignon exhibited 302 significant features differences for total volatiles among which 215 features were present in higher intensity in Cabernet Sauvignon than Norton. Similarly, 87 features were significantly higher in intensity in Norton grapes. The principal component analysis using the significant features demonstrated no overlap between the two groups indicating Norton and Cabernet Sauvignon very different in terms of the total volatile composition (Figure 2.2B). PC1 explained $49.4 \%$ of the variation and demonstrated mostly separation due to genotype, whereas PC2 explained around $14.38 \%$ of the variation between samples within the genotype (Figure 2.2 B). The clear separation of the two cultivars in terms of total volatiles again indicated that their genetic differences are driving the differences in the volatile composition. Again, these cultivars didn't show distinct differences in terms of location as well as the stage of ripening (veraison, pre-harvest, harvest, post-harvest) (data not shown).

\subsection{Wine volatiles:}

Even though the wines of Norton and Cabernet Sauvignon used in the analysis were commercial wines from different states of the United States as well as Australia (Supplemental Table 6), the PCA scores plot demonstrated differences between the aroma of Norton and Cabernet Sauvignon wines. We were able to identify 1064 features that characterized the populations, of them 403 were significantly different at 5\% level of significance and have greater than the 1.5 -fold change between Norton and Cabernet 
Sauvignon. About 207 features had a higher intensity in Cabernet Sauvignon wines, whereas, 196 features had a higher in Norton wines. PCA analysis using the significant features between the two cultivars indicated that the commercial wines of these two cultivars exhibited differences in the volatile composition (Fig 2.2C). PC1 explained $40.42 \%$ of the variation, mostly the variation between two cultivars, whereas PC2 explained $12.17 \%$ of the variation. The two cultivars did show clear isolation in terms of the wine volatiles and did not show any differences in terms of the winery, vintage as well as location. Norton wines had a greater distribution compared to Cabernet Sauvignon which might be due to stylistic variation in winemaking styles of Norton.

\subsection{Identification of compounds from significant features:}

Using the metabolomics-based pipeline, the significant features driving differences between cultivars were identified, however, for biological interpretation, the identification of the compounds represented by those features is important. The putative compounds were identified by comparing mass spectra of the features with that of the database using the NIST library database, comparing retention indices with that of literature, and comparing the mass spectra with the standards available in the laboratory. We were able to identify a total of 165 putative compounds among which 83,41 and 70 putative compounds are free, bound, and wine volatile respectively (Supplemental Table 4). Of these 13 compounds were common in the free, bound, and wine volatiles samples. The compounds identified in this way were further screened to make sure they were likely sample derived and not artifacts of sample preparation or analysis. Positive relation

of features to compound spectra in the database was not possible for many features due to limitations of the database, potential co-elution causing a combined spectra, or poor 
spectral differentiation within certain common classes of compounds (e.g. monoterpenes and esters). Additionally, in some cases, identification was not possible due to the lack of available authentic standards. For the compounds whose authentic standards were available, proper calibration and internal standards were used to minimize the run to run variation. Many compounds such as fatty acid alcohols, norisoprenoids, terpenes, esters, terpinols, and acids were found (Table 2.1).

\subsection{Quantitation of the important volatile compounds:}

Based on the results from the untargeted compound identification, we quantified 20 volatile compounds for free and total for which authentic standards were available in consideration of the price and importance of the compounds. For wine volatiles, 22 volatile compounds were quantified. The compounds quantified and their concentration in free, total, and wine volatiles are shown in (Table 2.1) and (Supplemental Table 5). PCA using the quantified compounds along with the biplot also demonstrated separation based on the cultivars for all free, total, and wine volatiles (Figure 2.4). Compounds such as $\beta$-Ionone, Methyl hexanoate, Terpinolene, Ethyl hexanoate, and $\beta$-Cyclocitral were found in higher concentration in Cabernet Sauvignon as free volatiles, whereas, Norton has a higher amount of Eugenol, Methyl Salicylate, 2-phenylethyl alcohol, and 1Nonanol as free and total volatiles (Figure 2.3A and Figure 2.4B). Total Nerol is found in higher concentrations in Cabernet Sauvignon, unlike free Nerol which is higher in Norton (Table 2.1).

The PCA scores plot of wine volatiles showed some overlap between Norton and Cabernet Sauvignon wines (Figure 2.4E) which might be due to variation in winemaking and origin of grapes of the commercial wines sampled. We used commercial Norton and 
Cabernet Sauvignon wines and did not control for the location, yeast strain used, the winemaking process, and the use of different types of oak. The compounds that are found to overlap with wines from both cultivars are mostly esters such as methyl octanoate, ethyl nonanoate, and isoamyl hexanoate that are formed during the process of fermentation (Figure 2.4F). Higher concentrations of Methyl salicylate, $\beta$-Damascenone, TDN, $\beta$-Linalool, and Ethyl nonanoate was present n Norton wines, whereas, a significantly higher amount of Isoamyl acetate, Ethyl octanoate, and Ethyl decanoate were found in Cabernet Sauvignon wines (Table 2.1). Compounds such as isoamyl acetate, Ethyl decanoate, phenylethyl ethanol were found in higher concentrations in Cabernet Sauvignon wines than Norton wines (Table 2.1). Norton wines had higher concentrations of p-Cymene, 1-Hexanol, Linalool, 1-Octanol (Figure 2.3C), Methyl Salicylate (Figure 2.5C), $\beta$-Damascenone (Figure 2.6C), TDN (Figure 2.7C) in comparison to Cabernet Sauvignon wines. The average concentration of $\beta$-Damascenone in Cabernet Sauvignon wines was quantified average $4.34 \mathrm{ug} / \mathrm{L}$, whereas Norton wines contained an average $7.26 \mathrm{ug} / \mathrm{L} \beta$ - Damascenone (Table 2.1). $\beta$-Damascenone is a very important C13-norisoprenoid that is noted for its baked apple, honey, and fruity flavor. This compound was abundant almost two times in Norton compared to Cabernet Sauvignon (Figure 2.6). Methyl salicylate was observed to be much higher in Norton (up to $18 \mathrm{ug} / \mathrm{L}$ ) in comparison to almost non-existent in Cab (Figure 2.5). Ethyl decanoate is present in Cabernet Sauvignon wines in the mean of $139.79 \mathrm{ug} / \mathrm{L}$, whereas, in Norton wines, its mean value is $88.3 \mathrm{ug} / \mathrm{L}$ (Table 2.1). 


\subsection{Characterizing F1 population of Norton and Cabernet Sauvignon with the identified compounds:}

The F1 mapping population developed from Norton and Cabernet Sauvignon cross were screened for free and bound volatiles using the compounds identified and confirmed in 2016 (Table 2.2). We quantified 14 compounds in the F1 population that exhibited clear differences in the Norton and Cab (Table 2.1 and Table 2.2). To identify useful potential phenotypes, we first identified the compounds that were different in the parents and phenotype those compounds in the progeny i.e. F1 population. Due to limitations of the fruit in each vine, wines could not be made. The boxplot showing the distribution of free and total volatiles in the F1 population is shown in (Figure 2.8A and B). All the compounds exhibited a continuous variation in the progeny i.e. the F1 population, which is typical for a polygenic inheritance as aroma compounds are complex and known to be governed by polygenes with small effect. We found many F1 genotypes that had a higher concentration of volatile compounds than the parents as well as many genotypes that had a lower concentration of compounds than the parents. In other words, transgressive segregation occurred in both directions. Aroma compounds exhibited different frequency distributions in the F1 population indicating different modes of inheritance (Figure 2.9). The results indicate that the F1 population can be used for genetic analysis to uncover the regions or genes regulating the important aroma compounds. QTL analysis using the combined SSR and GBS map of the population (Sapkota et al. 2019) will be used to understand the genetics of potential aroma phenotypes. 


\section{Discussion:}

Chemical analysis of grape juice and wine has been performed for more than 50 years to determine the limited number of compounds using gas chromatography mass spectrometry, which allowed for the determination of metabolites present in high concentration. Most of these studies were focused on the study of a selected number of compounds that were known to be significant for Vitis vinifera. Very limited studies have been conducted that focused on the disease tolerant hybrid grapes such as Norton that are important cultivars in the Mid-Western United States (Sun et al. 2011b; Slegers et al. 2015). Untargeted or metabolomics-based approaches thus provide the ability to identify as many metabolites as possible or at least those with certain properties without the requirement of pre-information about the compounds due to the comprehensive and unbiased nature of untargeted metabolomics. This approach has proved invaluable in situations where stark differences are expected such as comparing mutant and wild type populations, infected and healthy individuals (Schueuermann et al. 2016). To comprehensively characterize aromas in understudied hybrid cultivars, it is necessary to first be inclusive in the initial selection of analytes of interest. Thus, the metabolomicsbased approach followed by quantitation of the compounds was used to characterize the volatile profile in Norton grapes and its comparison with the widely cultivated vinifera wine grape, Cabernet Sauvignon.

From XCMS online, 924, 793, and 1064 metabolic features were identified for free volatiles, total volatiles, and wine volatiles respectively that define differences between Norton and Cabernet Sauvignon, which is widely accepted values in

metabolomics studies (Schueuermann et al. 2016; Šuklje et al. 2019). Only statistically 
significant features based on ANOVA and FDR correction with a fold change greater than 1.5 were further used for analysis as many features can be the noise or artifacts of the column. To get a meaningful interpretation from the features in terms of biological functionality, compound identification was done from the features, from which 165 compounds were identified which compares favorably with 99 compounds detected in Pinot noir due to barrel storage (Schueuermann et al. 2016) and 175 features due to ripening differences in Shiraz grapes (Šuklje et al. 2019) and 115 metabolites due to berry development in Cabernet Sauvignon and Merlot grapes (Cuadros-Inostroza et al. 2010). Based on the significance of the identified compound to grape and wine aroma, standard availability, and cost, we confirmed and quantified a total of 33 compounds in free, total, and wine volatiles. Metabolomics based approach thus helped in narrowing down the most significant compounds from an extensive list of features detected.

Berry skin had been found to contain the largest concentration of volatiles than pulp (Callejon et al. 2012; Canuti et al. 2009; Slegers et al. 2015). In our study, a conscious decision was made to focus on berry skin as glucosidase cannot work in a high sugar environment when pulp and skin both used. Centrifugation was effectively used to remove most of the juice from the skin so that enzymatic digestion can be effective.

While metabolites provide phenotypic information of cells in response to different environmental and genetic changes, the genetics of the cultivar is the most significant factor impacting the volatile composition in grapes and wine (Ghaste et al. 2015). Our results indicated that Norton and Cabernet Sauvignon grapes and wines are distinct from each other in their volatile composition although the grapes were sourced from different 
locations within Missouri and Virginia and harvested at different stages of ripening (Supplemental Table 4). Similarly, wines analyzed in our study were sourced from different wineries from the USA and Australia (Supplemental Table 6). The intent in using diverse samples from different years and regions was to identify those compounds that encompassed a consistent and meaningfully large difference between Norton and Cabernet Sauvignon. A controlled study between Norton and Cabernet Sauvignon, may allow for the identification of additional traits and minimize the environmental variations such as soil, rainfall, water availability, sunshine as well as variation in viticultural operations and winemaking practices. All these environmental, viticultural and winemaking practices have been found to have a subtle but significant impact on the berry and wine volatile composition (Alessandrini et al. 2017; Main and Morris 2008; Hernandez-Orte et al. 2015). However, due to the climatic conditions of Missouri, it was not feasible to grow both the cultivars in the same locations. Also, this study aimed to identify the strongest differences that appear between two cultivars despite the muddy sampling and differences. Our results thus demonstrate that genetic differences were the most significant driver of aroma differences in two cultivars.

From the quantified compounds, we did not find any compound that was present in one cultivar and absent in the other, but a huge variation in the concentration of some compounds (Table 2.1). The presence of some compounds above odor threshold in Norton such as Eugenol (55.8 ug/L) and present well below odor threshold (3ug/L) in Cabernet Sauvignon $(0.425 \mathrm{ug} / \mathrm{L})$ might be responsible for the distinct aroma in the two cultivars. Eugenol is a phenylpropanoid derived volatile compound which is described as having a pungent, minty, and clove oil aroma widespread in many plant species. Eugenol 
is more abundant in non-vinifera species, mostly in its bound form (Sun et al. 2011a; Ghaste et al. 2015). However, our results indicated that Norton had higher free Eugenol than its bound fraction which might be due to multiple enzyme activities (esterase, oxidase in addition to glucosidase) of the Rapidase enzyme (Hampel et al. 2014). The presence of Eugenol at such a higher concentration in Norton might explain the minty aroma in some Norton wines.

Although not present above the odor threshold, Methyl salicylate was found to be higher in Norton in free, total, and wine volatiles. Methyl salicylate is a methylated form of a stress hormone Salicylic acid (SA) associated with biotic stress caused by obligate pathogens. SA is a benzoic acid derivative, generally involved in the activation of defense responses against biotrophic (which keeps the cell alive) and hemi biotrophic (which initially keep the cell alive but kill them at later stages) pathogens. Methyl ester of salicylic acid, methyl salicylate can act as volatile SAR inducing signals transmitted to distant plant parts or even the surrounding plant parts. SA being immobile, methyl salicylate has been known as one of the mobile signaling molecules. Methylation inactivates SA while increasing its membrane permeability and volatility, allowing more effective long-distance transport of the defense signal (Dempsey et al. 2011). Earlier studies have found that Norton leaves accumulate high levels of salicylic acid and SArelated defense genes in comparison to Cabernet Sauvignon which may have contributed Norton grape with a robust innate defense system against pathogens (Fung et al. 2008). The higher proportion of methyl salicylate in grapes and wines of Norton shows the presence of defense mechanism, systemic acquired resistance (SAR) in Norton, which makes these berries resistant to many grape pathogens. This may be the reason why 
Norton grapes are resistant to many disease pathogens, in comparison to Cabernet Sauvignon, which had lower levels of methyl salicylate. While glycosylated methyl salicylate had been found in some vinifera berries (Carlin et al. 2019), our results indicated that most of the methyl salicylate was present in free form. The reason for this might be the prevalence of higher free form in Norton grapes, which need to be investigated further or might be due to esterase side activity of the Rapidase enzyme in addition to glycosidase activity. Rapidase had been found to have some esterase activity (Hampel et al. 2014). The frequency distribution of methyl salicylate in the F1 population was continuous, suggesting multiple genes regulation, ranging up to $52.50 \mathrm{ug} / \mathrm{L}$ of free volatiles and $157.25 \mathrm{ug} / \mathrm{L}$ of total volatiles.

$\beta$-Damascenone was found to be two times more abundant in Norton than in Cabernet Sauvignon for all free, total, and wine volatiles, although they are present above their odor threshold in both cultivars (Figure 2.6 and Table 2.1). In the F1 population, free $\beta$-Damascenone demonstrated continuous distribution, with some F1's having concentrations up to $42 \mathrm{ug} / \mathrm{L}$. $\beta$-Damascenone is a very important carotenoid derived C13-norisoprenoid that is noted for its baked apple, honey, and fruity flavor. Typically, in vinifera red wines, $\beta$-Damascenone is present at or below the odor threshold $(1-2 \mathrm{ug} / \mathrm{L})$ and was found to have an indirect impact on red wine aroma by enhancing the threshold of fruity esters (ethyl cinnamate and ethyl caproate) and decrease the green bell pepper aroma (Pineau et al. 2007; Sefton et al. 2011). Earlier studies have reported a higher amount of $\beta$-Damascenone in non-vinifera cultivars up to $30 \mathrm{ug} / \mathrm{L}$ in St. Croix (Slegers et al. 2015; Slegers et al. 2017). The higher amount of this compound in grapes and wines are often correlated with more fruity flavors (Sefton et al. 2011). This suggests that two 
times higher amount of this compound might be responsible for the overwhelming fruity aroma of Norton grapes and wines and might be affecting positively and negatively to the Norton grape and wine aroma. The levels of $\beta$-Damascenone in wines were lower than that of total volatiles and the decrease in its concentration in wine might be due to its reaction with sulfites as well as molecular rearrangements in acidic conditions (Sefton et al. 2011).

Free 1,1,6-trimethyl-1,2-dihydronapthalene (TDN) was found to be higher in Norton (7.35 ug/L) and Cab (2.66 ug/L) (Figure 2.7). Also, a C13 norisoprenoid derived from carotenoid degradation, TDN has a very low sensory threshold ( $2 \mathrm{ug} / \mathrm{L})$ and thus can impart negative attribute to wine at higher concentration (Sacks et al. 2012). It is a characteristic varietal character of aged Riesling wine (concentration up to $50 \mathrm{ug} / \mathrm{L}$ ), however, lower than its threshold in other vinifera cultivars, with exception of $6.4 \mathrm{ug} / \mathrm{L}$ in Cabernet Franc (Sacks et al. 2012; Kwasniewski et al. 2010). This compound was not reported in interspecific hybrids in previous studies. In the F1 population of Norton by Cab cross, the concentration of TDN was found to be higher than that of any parents, averaging $6.89 \mathrm{ug} / \mathrm{L}$ for free and $15.70 \mathrm{ug} / \mathrm{L}$ for total volatiles. We observed some F1 genotypes with much higher concentrations (up to $64 \mathrm{ug} / \mathrm{L}$ total TDN), showing transgressive segregation. It should be noted that while Cab had TDN concentrations lower than the odor threshold, it had Cabernet Franc as one of its parents.

Monoterpenes such as $\beta$-caryophyllene, $\beta$-linalool, $\mathrm{p}$-Cymene, and Nerol was found to be more abundant in Norton than Cabernet Sauvignon. Terpenes, also known as isoprenoids are one of the important secondary metabolites in plants important for the plant's resistance to diseases and pests. They are an important volatile constituent in 
grape berries present in free and bound form and biosynthesized from the primary metabolites through the mevalonic pathway and methylerythritol pathway in cytosol and chloroplast respectively. It has been found that the de novo synthesis of monoterpenes and sesquiterpenes occurs via the octadecanoid signaling cascade using methyl jasmonate which is involved in plant defense (Schwab and Wüst 2015). The abundance of monoterpenes and sesquiterpenes in Norton higher than Cabernet Sauvignon grapes and wines indicated the probable role of regulation of stress hormone, jasmonic acid and disease resistance nature of Norton.

We observed continuous distribution of the marker aroma compounds in the F1 population indicating polygenic trait. Although aroma in grapes and wine is a highly appreciated organoleptic attribute, little is known about the inheritance patterns of the aroma traits and the main compounds associated with it. Few genetic studies have been done to uncover the genetic regions underlying the development and formation of some aroma compounds in apples (Vogt et al. 2013; Dunemann et al. 2009), grapes (Doligez et al. 2006; Battilana et al. 2009; Guillaumie et al. 2013) and have demonstrated variability and heritability of individual aroma compounds. Few genetic studies in grapes focused using quantitative trait loci (QTL) analysis identified QTLs for muscat flavor on three linkage groups (Doligez et al. 2006) and IBMP in linkage group 2 and 3 (Guillaumie et al. 2013) and found to be governed by multiple complementary genes and modifier genes. While aroma traits are known to be complex, the phenotyping of aroma compounds in population is even difficult as they interact with environmental factors such as climate, season, vintage, and location. In our study, we found that most of the free and total aroma compounds exhibited a distribution in the F1 population which 
indicated that they are under genetic control, Generally, the levels of compounds in the F1 population are located within and outside the range given by the two parental cultivars, showing transgressive segregation. The frequency distributions in the F1 population for individual aroma compound is different indicating different modes of inheritance. This might be due to the complex biochemical pathways from which volatiles are derived. As grapes are heterogenous, the F1 genotypes have a good probability of showing some regressive traits that were not seen in their parents but in the grandparents from which the parents were developed. For example, Cabernet Sauvignon had Cabernet Franc as one of its parents and some compounds which were not present or present below odor threshold in Cabernet Sauvignon but present in its parents can now appear in its offspring, an example being TDN. Thus, using the aroma compounds, QTL analysis can be useful method to identify genetic regions underlying QTL associated with the trait. Identifying QTLs will help to cumulate favorable alleles at several loci to reach stable aroma phenotype that can be used in breeding new grape cultivars that are both disease resistant as well as have desired aroma character.

There is currently great interest in breeding new grapevine varieties resistant to diseases of major economic importance in viticulture as modern Vitis vinifera cultivars are disease susceptible and spraying fungicides and pesticides are not economically and environmentally sustainable. These breeding programs based primarily on resistance characters might need to consider the quality-related traits as many hybrids that are disease and pest resistant are not satisfactorily accepted by growers and in terms of consumer preference due to their different flavors. There is no value in producing grapes new varieties until they taste good. Thus, it might be high time to take the volatile profile 
of parents into serious consideration before making crosses to develop breeding populations. Finding meaningful aroma phenotypes or traits that show differentiation in parents and using them in genetic analysis of every breeding population will be a better approach in the grape breeding pipeline to utilize the limited resources and time. Grapes being a perennial crop and having a long juvenile period is both expensive and timeconsuming to maintain and phenotype a breeding population requires more labor and resources, which makes it even more essential to consider the parental volatile profile before making a cross. This might improve the consumer acceptance of new environmentally sustainable hybrid cultivars of grapevines. Also, the development of molecular markers linked to genes for key aroma compounds will be an important step towards a future marker-assisted breeding.

\section{Conclusions:}

In conclusion, the metabolomics-based approach was used to identify aroma differences in Norton and Cabernet Sauvignon free and total volatiles as well as wines. Both the cultivars showed significant differences in the aroma profile, which suggested that genetics of the plant cultivar plays an important role in the aroma profile of grapes and wines. The features identified using the untargeted approach were further narrowed down to identify putative compounds and a total of 33 aroma compounds were quantified in free, total, and wine volatiles. Norton was characterized by a higher abundance of

methyl salicylate, eugenol, $\beta$-Damascenone, Nerol, TDN, p-Cymene, whereas Cabernet Sauvignon was characterized by a higher amount of $\beta$-Ionone, Ethyl decanoate and isoamyl acetate in wines. Norton berries and wine were found to contain a higher concentration of compounds that are related to stress or defense. The phenotyping of the 
F1 population of Norton and Cabernet Sauvignon for free and bound volatiles demonstrated huge variability for the identified compounds. Some of the F1 genotypes demonstrated a higher concentration of compounds than the parents, whereas, we also observed lower concentration of some compounds in F1 genotypes than either of the parents. The future work for this study will be to find out the regions or genes in chromosomes that regulate the concentration of these compounds by using QTL mapping. Finding the genes or location of these genes will help breeders in developing sustainable genotypes that have suitable aroma qualities along with the disease resistance traits.

\section{Acknowledgments:}

We thank Dr. Chin Feng Wang from Missouri State University for making the cross of Norton and Cabernet Sauvignon and providing us the opportunity to sample the F1 population. We thank Dr. Tony Wolf from Virginia Tech University for providing us Cabernet Sauvignon grapes for analysis.

\section{References}

Ambers CP (2013) A historical hypothesis on the origin of the Norton grape. Journal of Wine Research 24 (2):85-95. doi:10.1080/09571264.2012.747087

Biasoto ACT, Netto FM, Marques EJN, da Silva MAAP (2014) Acceptability and preference drivers of red wines produced from Vitis labrusca and hybrid grapes. Food Research International 62:456-466. doi:10.1016/j.foodres.2014.03.052

Chisholm MG, Guiher LA, Vonah TM, Beaumont JL (1994) Comparison of some FrenchAmerican hybrid wines with White Riesling using gas chromatography-olfactometry. American journal of enology and viticulture 45 (2):201-212 
Commisso M, Strazzer P, Toffali K, Stocchero M, Guzzo F (2013) Untargeted metabolomics: an emerging approach to determine the composition of herbal products. Computational and structural biotechnology journal 4 (5):e201301007

Cuadros-Inostroza A, Giavalisco P, Hummel J, Eckardt A, Willmitzer L, Peña-Cortés H (2010) Discrimination of wine attributes by metabolome analysis. Analytical Chemistry 82 (9):3573-3580

de Pinho PG, Bertrand A (1995) Analytical determination of furaneol (2, 5-dimethyl-4-hydroxy-3 (2H)-furanone). Application to differentiation of white wines from hybrid and various Vitis vinifera cultivars. American journal of enology and viticulture 46 (2):181-186

De Vos RC, Moco S, Lommen A, Keurentjes JJ, Bino RJ, Hall RD (2007) Untargeted large-scale plant metabolomics using liquid chromatography coupled to mass spectrometry. Nature protocols 2 (4):778

Dempsey DMA, Vlot AC, Wildermuth MC, Klessig DF (2011) Salicylic Acid biosynthesis and metabolism. The arabidopsis book 9:e0156-e0156. doi:10.1199/tab.0156

Ebeler SE (2001) Analytical Chemistry: Unlocking the Secrets of Wine Flavor. Food Reviews International 17 (1):45-64. doi:10.1081/fri-100000517

Ebeler SE, Thorngate JH (2009) Wine chemistry and flavor: looking into the crystal glass. J Agric Food Chem 57 (18):8098-8108. doi:10.1021/jf9000555

Ferreira V, López R, Cacho JF (2000) Quantitative determination of the odorants of young red wines from different grape varieties. Journal of the Science of Food and Agriculture 80 (11):1659-1667

Fung RW, Gonzalo M, Fekete C, Kovacs LG, He Y, Marsh E, McIntyre LM, Schachtman DP, Qiu W (2008) Powdery mildew induces defense-oriented reprogramming of the transcriptome in a susceptible but not in a resistant grapevine. Plant physiology 146 (1):236-249 
Ghaste M, Narduzzi L, Carlin S, Vrhovsek U, Shulaev V, Mattivi F (2015) Chemical composition of volatile aroma metabolites and their glycosylated precursors that can uniquely differentiate individual grape cultivars. Food Chem 188:309-319. doi:10.1016/j.foodchem.2015.04.056

Gowda H, Ivanisevic J, Johnson CH, Kurczy ME, Benton HP, Rinehart D, Nguyen T, Ray J, Kuehl J, Arevalo B, Westenskow PD, Wang J, Arkin AP, Deutschbauer AM, Patti GJ, Siuzdak G (2014) Interactive XCMS Online: Simplifying Advanced Metabolomic Data Processing and Subsequent Statistical Analyses. Analytical Chemistry 86 (14):69316939. doi:10.1021/ac500734c

Gunata Z, Vallier M, Sapis J, Baumes R, Bayonove C (1994) Enzymatic synthesis of monoterpenyl $\beta$-D-glucosides by various $\beta$-glucosidases. Enzyme and microbial technology 16 (12):1055-1058

Hampel D, Robinson AL, Johnson A, Ebeler SE (2014) Direct hydrolysis and analysis of glycosidically bound aroma compounds in grapes and wines: comparison of hydrolysis conditions and sample preparation methods. Australian journal of grape and wine research 20 (3):361-377

Hjelmeland AK, Ebeler SE (2014) Glycosidically Bound Volatile Aroma Compounds in Grapes and Wine: A Review. American Journal of Enology and Viticulture 66 (1):1-11. doi:10.5344/ajev.2014.14104

Hjelmeland AK, Zweigenbaum J, Ebeler SE (2015) Profiling monoterpenol glycoconjugation in Vitis vinifera L. cv. Muscat of Alexandria using a novel putative compound database approach, high resolution mass spectrometry and collision induced dissociation fragmentation analysis. Anal Chim Acta 887:138-147. doi:10.1016/j.aca.2015.06.026

Jackson RS (2008) Wine science: principles and applications. Academic press,

Kolor MG (1983) Identification of an important new flavor compound in Concord grape: ethyl-3mercaptopropionate. Journal of Agricultural and Food Chemistry 31 (5):1125-1127 
Mateo J, Jiménez M (2000) Monoterpenes in grape juice and wines. Journal of chromatography A $881(1-2): 557-567$

Moyer J, Mattick L (1976) Determination of methyl anthranilate in wines. American Journal of Enology and Viticulture 27 (3):134-135

Narduzzi L, Stanstrup J, Mattivi F (2015) Comparing Wild American Grapes with Vitis vinifera: A Metabolomics Study of Grape Composition. J Agric Food Chem 63 (30):6823-6834. doi:10.1021/acs.jafc.5b01999

Nelson R, Acree T, Lee C, Butts R (1977) Methyl anthranilate as an aroma constituent of American wine. Journal of Food Science 42 (1):57-59

Nisbet MA, Martinson TE, Mansfield AK (2014) Accumulation and Prediction of Yeast Assimilable Nitrogen in New York Winegrape Cultivars. American Journal of Enology and Viticulture 65 (3):325-332. doi:10.5344/ajev.2014.13130

Parker M, Capone DL, Francis IL, Herderich MJ (2017) Aroma Precursors in Grapes and Wine: Flavor Release during Wine Production and Consumption. J Agric Food Chem. doi:10.1021/acs.jafc.6b05255

Pineau B, Barbe J-C, Van Leeuwen C, Dubourdieu D (2007) Which Impact for $\beta$-Damascenone on Red Wines Aroma? Journal of Agricultural and Food Chemistry 55 (10):4103-4108. doi:10.1021/jf070120r

Polaskova P, Herszage J, Ebeler SE (2008) Wine flavor: chemistry in a glass. Chem Soc Rev 37 (11):2478-2489. doi:10.1039/b714455p

Reisch BI, Goodman RN, Martens M-H, Weeden NF (1993) The Relationship Between Norton and Cynthiana, Red. Wine Cultivars Derived from Vitis aestivalis. American journal of enology and viticulture $44(4): 441-444$

Reisch BI, Owens CL, Cousins PS (2012) Grape. In: Fruit breeding. Springer, pp 225-262

Reynolds AG, Fuleki T, Evans W (1982) Inheritance of methyl anthranilate and total volatile esters in Vitis spp. American Journal of Enology and Viticulture 33 (1):14-19 
Robinson AL, Ebeler SE, Heymann H, Boss PK, Solomon PS, Trengove RD (2009) Interactions between wine volatile compounds and grape and wine matrix components influence aroma compound headspace partitioning. J Agric Food Chem 57 (21):10313-10322. doi:10.1021/jf902586n

Roullier-Gall C, Witting M, Gougeon RD, Schmitt-Kopplin P (2014) High precision mass measurements for wine metabolomics. Frontiers in chemistry 2:102

Sapkota S, Chen L-L, Schreiner K, Ge H, Hwang C-F (2015) A phenotypic study of Botrytis bunch rot resistance in Vitis aestivalis-derived 'Norton'grape. Tropical Plant Pathology $40(4): 279-282$

Schrimpe-Rutledge AC, Codreanu SG, Sherrod SD, McLean JA (2016) Untargeted Metabolomics Strategies - Challenges and Emerging Directions. Journal of The American Society for Mass Spectrometry 27 (12):1897-1905. doi:10.1007/s13361-016$1469-\mathrm{y}$

Schueuermann C, Khakimov B, Engelsen SB, Bremer P, Silcock P (2016) GC-MS Metabolite Profiling of Extreme Southern Pinot noir Wines: Effects of Vintage, Barrel Maturation, and Fermentation Dominate over Vineyard Site and Clone Selection. Journal of Agricultural and Food Chemistry 64 (11):2342-2351. doi:10.1021/acs.jafc.5b05861

Schwab W, Wüst M (2015) Understanding the Constitutive and Induced Biosynthesis of Monoand Sesquiterpenes in Grapes (Vitis vinifera): A Key to Unlocking the Biochemical Secrets of Unique Grape Aroma Profiles. Journal of Agricultural and Food Chemistry 63 (49):10591-10603. doi:10.1021/acs.jafc.5b04398

Sefton MA, Skouroumounis GK, Elsey GM, Taylor DK (2011) Occurrence, Sensory Impact, Formation, and Fate of Damascenone in Grapes, Wines, and Other Foods and Beverages. Journal of Agricultural and Food Chemistry 59 (18):9717-9746. doi:10.1021/jf201450q 
Shure KB, Acree TE (1994) Changes in the odor-active compounds in Vitis labruscana cv.

Concord during growth and development. Journal of agricultural and food chemistry 42 (2):350-353

Skinkis PA, Bordelon BP, Wood KV (2008) Comparison of monoterpene constituents in Traminette, Gewürztraminer, and Riesling winegrapes. American journal of enology and viticulture 59 (4):440-445

Slegers A, Angers P, Ouellet E, Truchon T, Pedneault K (2015) Volatile Compounds from Grape Skin, Juice and Wine from Five Interspecific Hybrid Grape Cultivars Grown in Quebec (Canada) for Wine Production. Molecules 20 (6):10980-11016. doi:10.3390/molecules200610980

Slegers A, Angers P, Pedneault K (2017) Volatile Compounds from Must and Wines from Five White Grape Varieties. Journal of Food Chemistry and Nanotechnology 03 (01). doi:10.17756/jfen.2017-031

Šuklje K, Carlin S, Stanstrup J, Antalick G, Blackman JW, Meeks C, Deloire A, Schmidtke LM, Vrhovsek U (2019) Unravelling wine volatile evolution during Shiraz grape ripening by untargeted HS-SPME-GC× GC-TOFMS. Food chemistry 277:753-765

Sun Q, Gates MJ, Lavin EH, Acree TE, Sacks GL (2011a) Comparison of odor-active compounds in grapes and wines from Vitis vinifera and non-foxy American grape species. Journal of agricultural and food chemistry 59 (19):10657-10664

Sun Q, Gates MJ, Lavin EH, Acree TE, Sacks GL (2011b) Comparison of odor-active compounds in grapes and wines from vitis vinifera and non-foxy American grape species. J Agric Food Chem 59 (19):10657-10664. doi:10.1021/jf2026204

Tautenhahn R, Patti GJ, Rinehart D, Siuzdak G (2012) XCMS Online: a web-based platform to process untargeted metabolomic data. Analytical chemistry 84 (11):5035-5039

Villamor RR, Ross CF (2013) Wine matrix compounds affect perception of wine aromas. Annual review of food science and technology 4:1-20 


\section{Figures:}

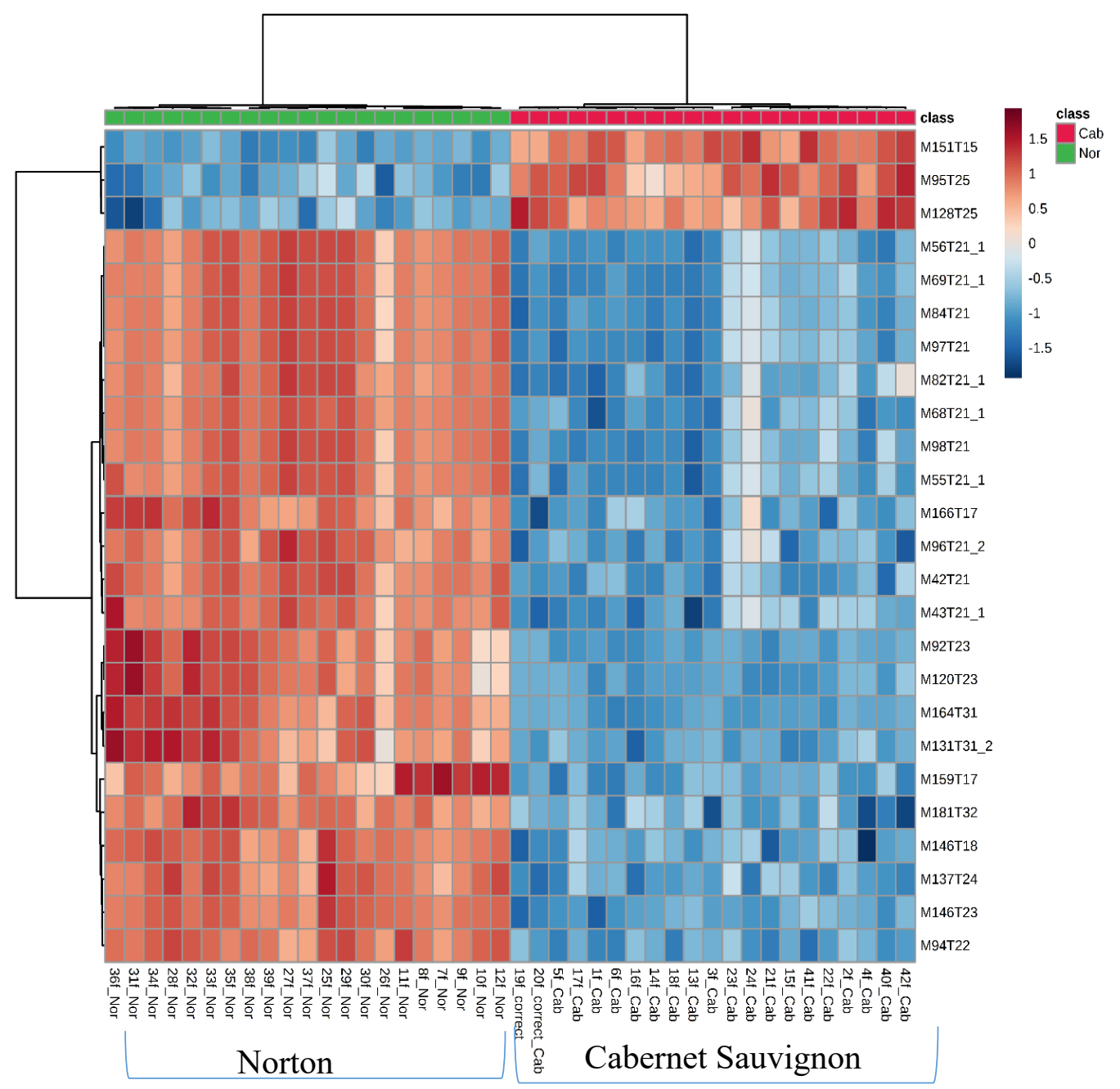

Figure 2.1: Heatmap of the top 25 most influential features for differentiating free volatiles between Norton and Cabernet Sauvignon berries. While only top contributors are shown the heatmaps were generated using all the features. The rows in the heatmap represent features $(\mathrm{M}(\mathrm{m} / \mathrm{z})$. T(time in minutes) and the columns indicate sample categories. The colors of the heatmap cells indicate the abundance of compounds across different samples. The color gradient, ranging from dark blue through white to dark red, represents low, middle and high abundance of a compound. 


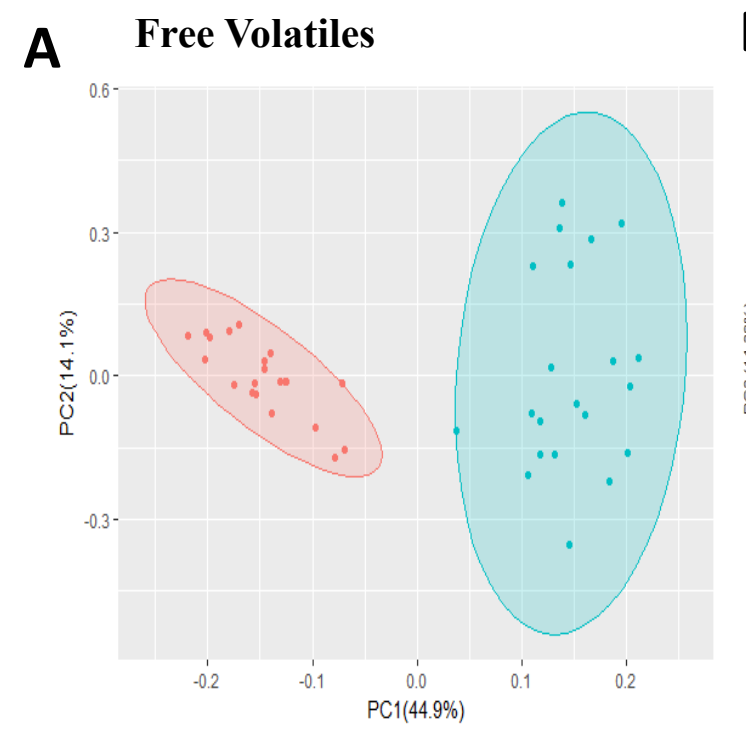

\section{B Total Volatiles}

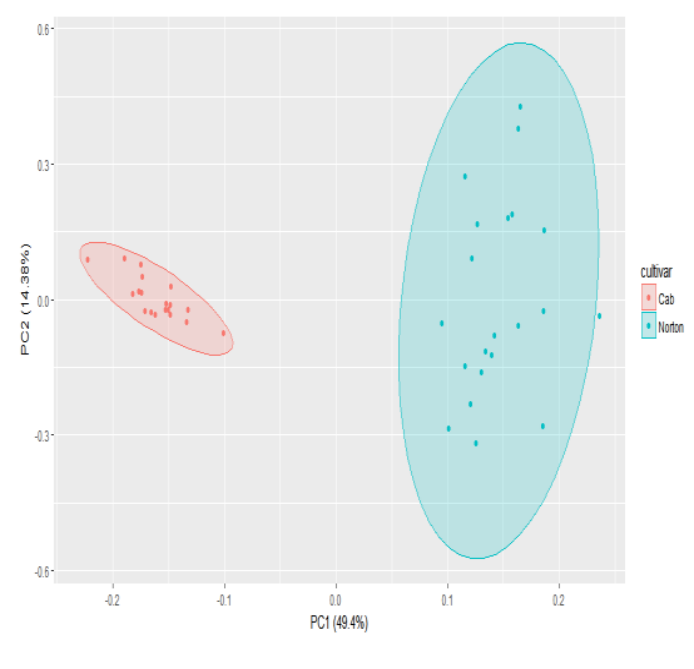

\section{Wine Volatiles}

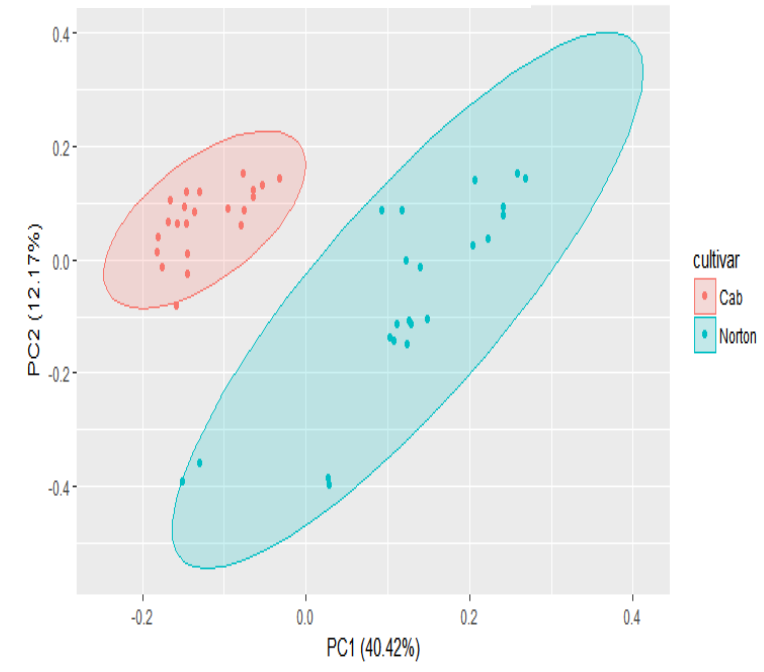

Figure 2.2: Principal components analysis (PCA) scores plot for the distribution of (A) free volatiles, (B) total volatiles and (C) wine volatiles features in Norton and Cabernet Sauvignon (Cab) grapes and wines. The ellipses show 95\% confidence interval. There is a separation based on cultivar for free, total and wine metabolic features. 


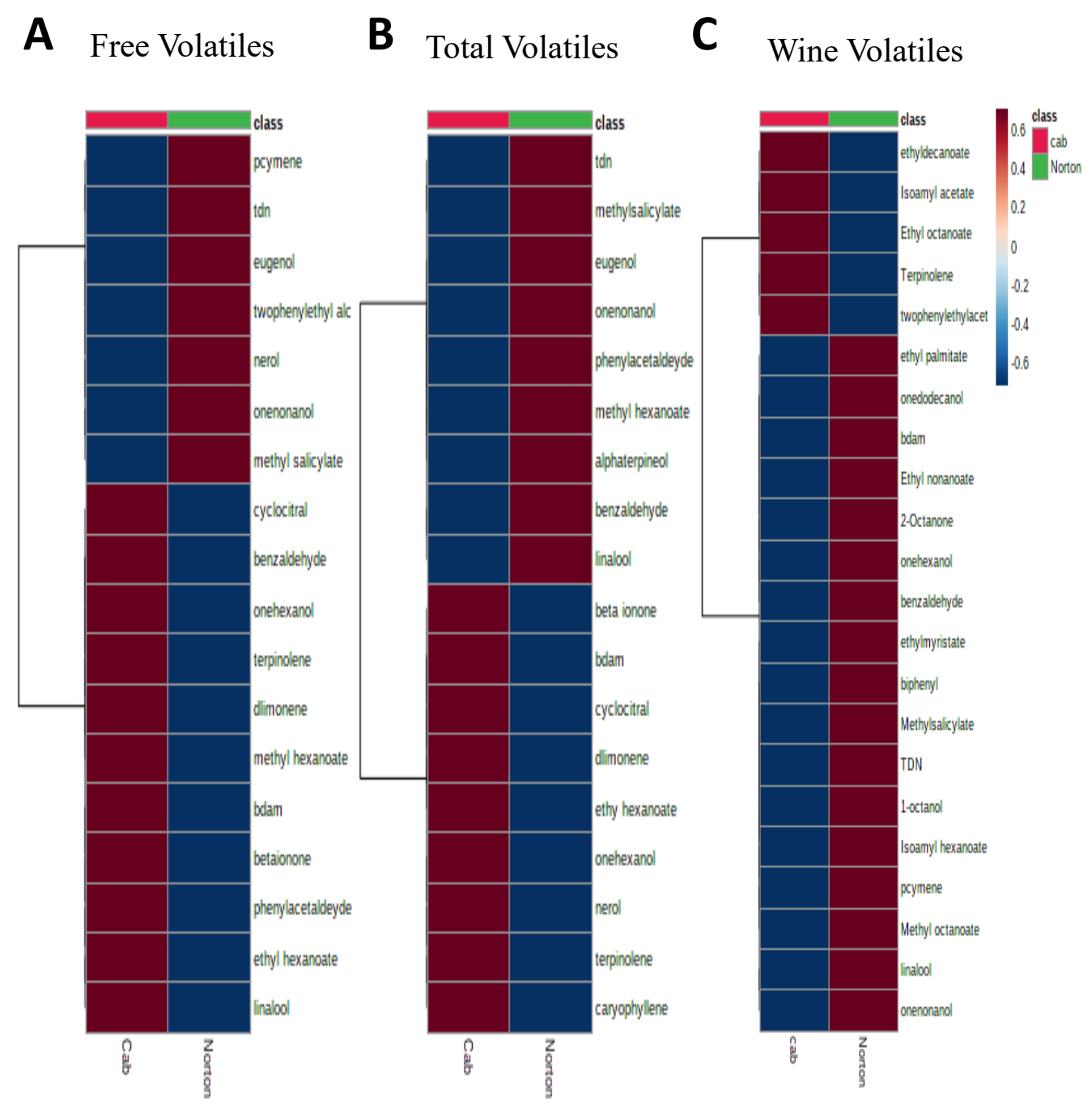

Figure 2.3: Heatmaps of the abundance of the volatiles in Norton and Cabernet Sauvignon (Cab) (A) free volatiles, (B) Total volatiles and (C) Wine volatiles. Heatmaps were created using average quantitative values based on 2-Octanol IS. The rows in the heatmap represent volatile compounds and the columns indicate cultivars. The colors of the heatmap cells indicate the abundance of compounds across different samples. The color gradient, ranging from dark blue through white to dark red, represents low, middle and high abundance of a compound. 
A
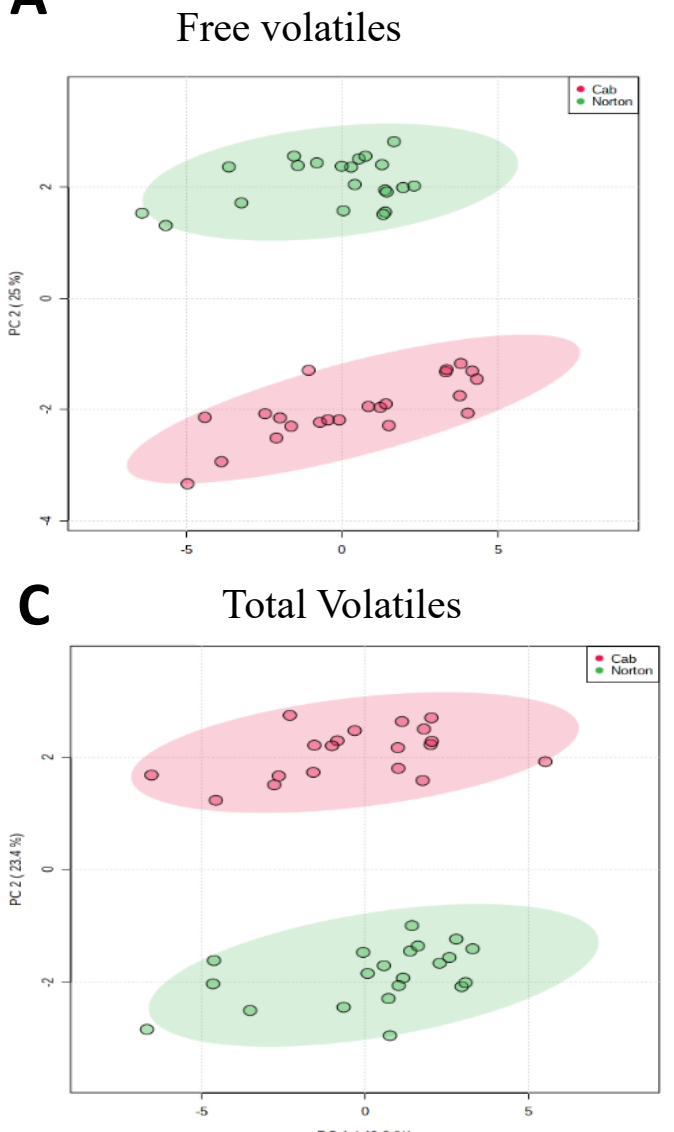

$\mathbf{E}$

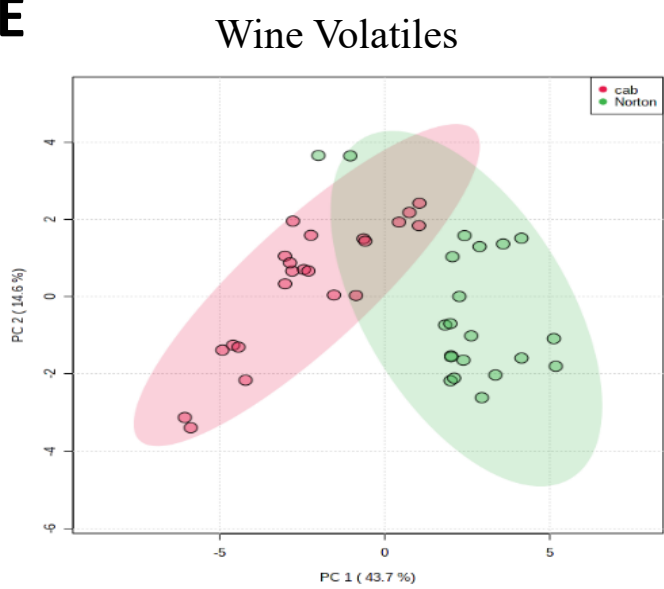

B Free volatiles

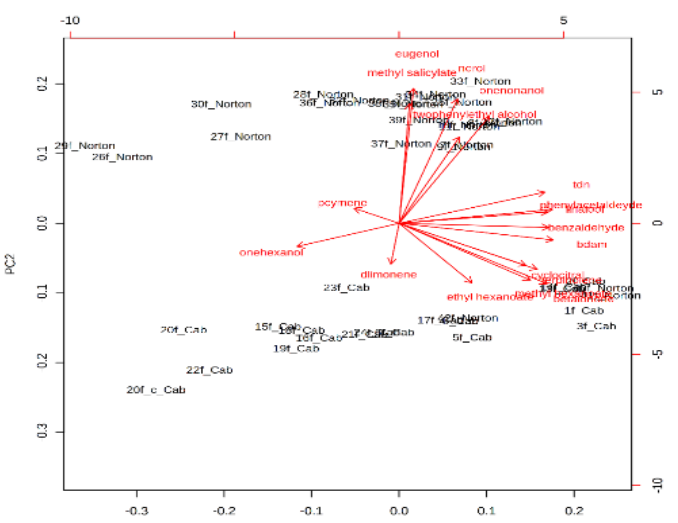

D Total Volatiles
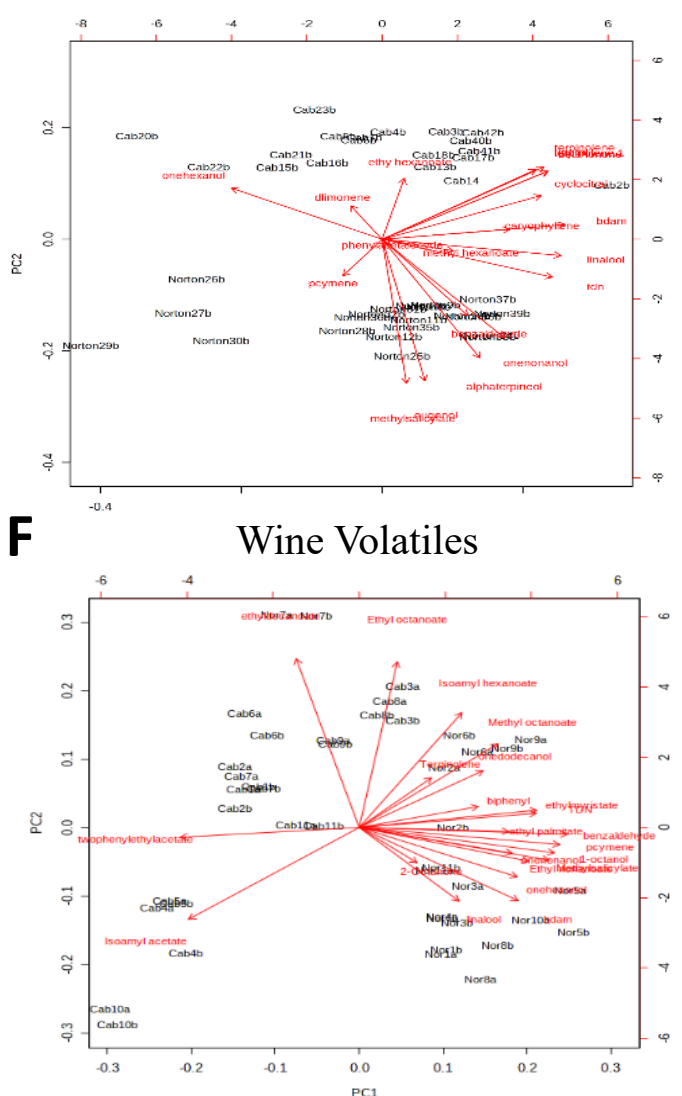

Figure 2.4: PCA scores map of the model obtained for (A and B) free, $(\mathrm{C}$ and $\mathrm{D})$ total and $(\mathrm{E}$ and $\mathrm{F})$ wine volatiles indicating the distinct separation of Norton and Cabernet Sauvignon for wine volatiles using quantitative data and the biplot showing volatiles contributing to the differences in Norton and Cab wine volatiles. 
A

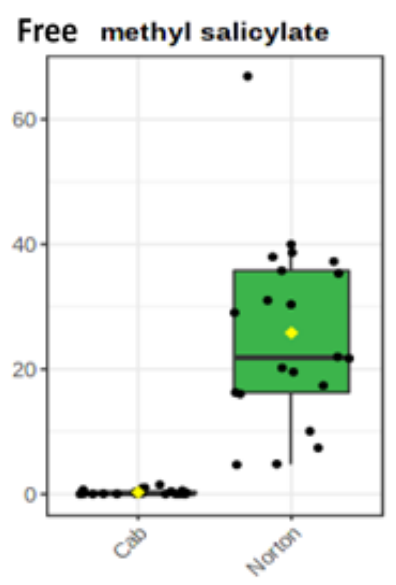

B

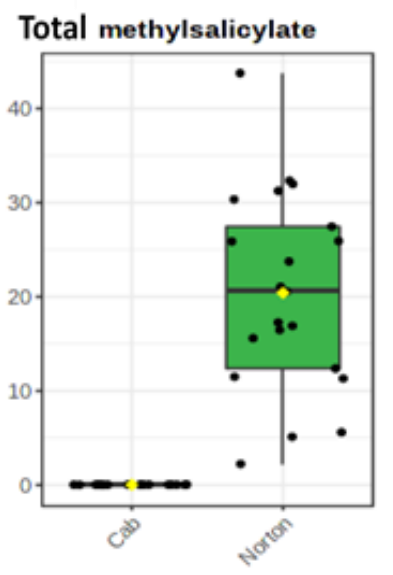

C

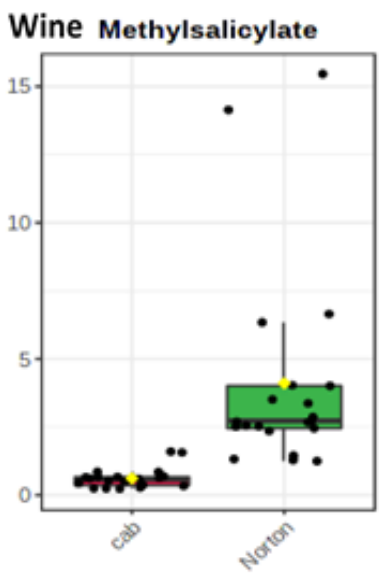

Figure 2.5: Boxplots showing the distribution of Methyl Salicylate in Norton and Cabernet Sauvignon (Cab) (A) free volatiles, (B) Total volatiles and (C) Wine volatiles. The $\mathrm{y}$-axis denotes concentration in $\mathrm{ug} / \mathrm{L}$ obtained semi-quantitatively relative to 2-Octanol.

A

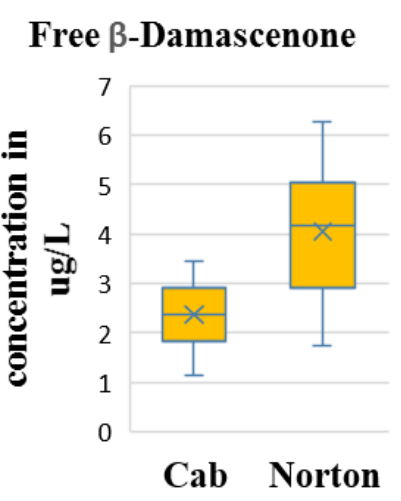

B

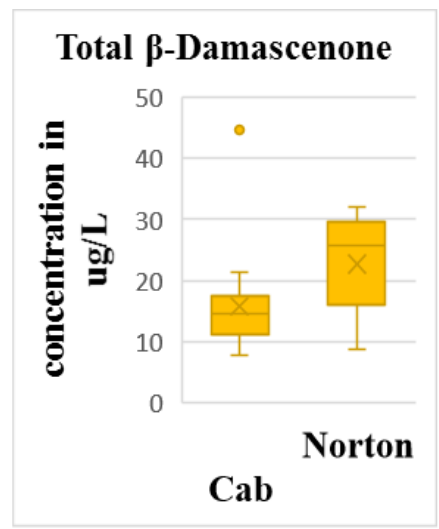

C

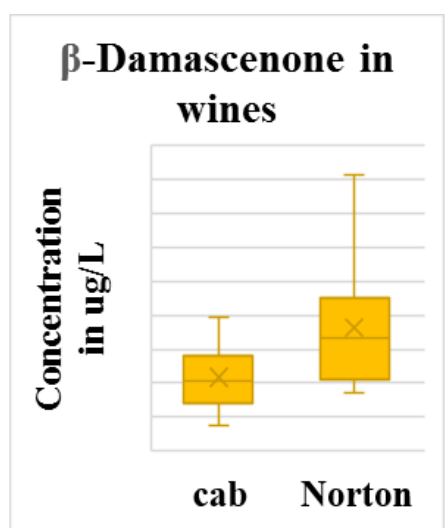

Figure 2.6: Boxplot showing the distribution of $\beta$-Damascenone in Norton and Cabernet Sauvignon (Cab) (A) Free, (B), total and (C) Wine volatiles. The yaxis denotes concentration in $\mathrm{ug} / \mathrm{L}$. 

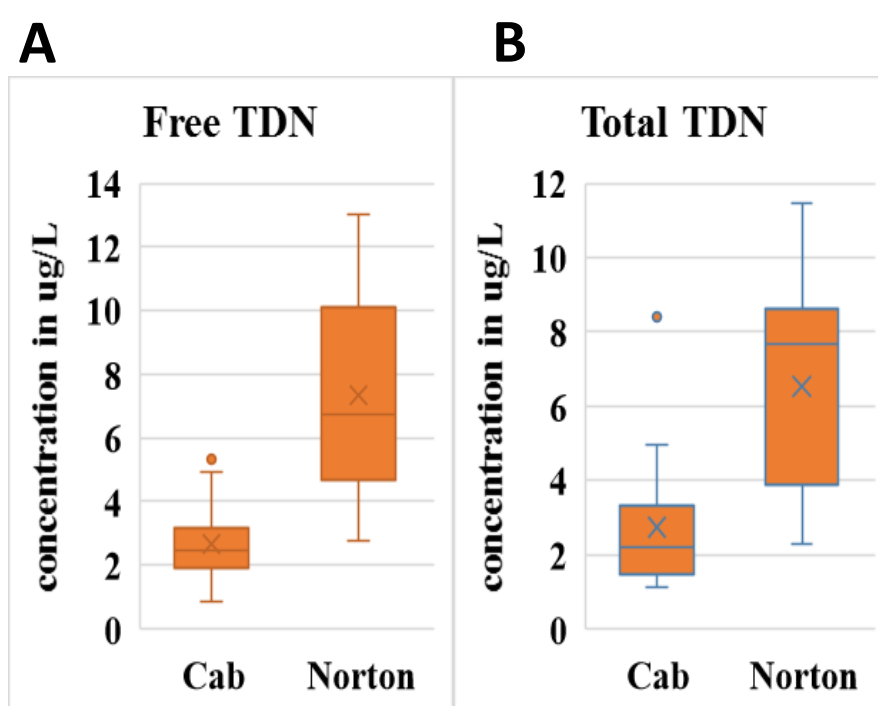

C

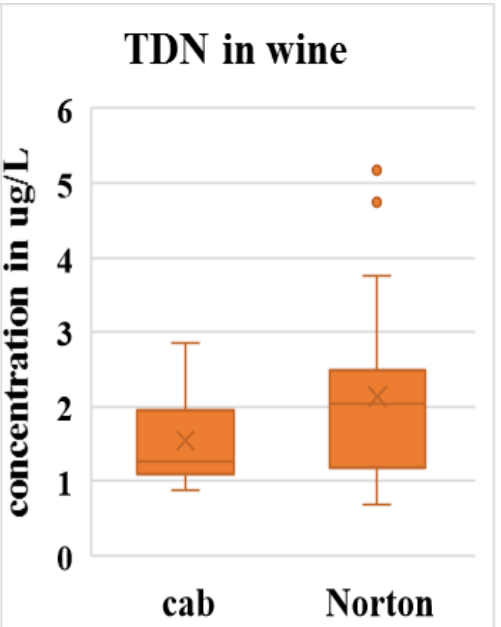

Figure 2.7: Boxplot showing the distribution of TDN in Norton and Cabernet Sauvignon (Cab) (A) Free, (B), total and (C) Wine volatiles. The y-axis denotes concentration in $u g / L$. 
A)

Free volatiles in $\mathbf{F} 1$ population

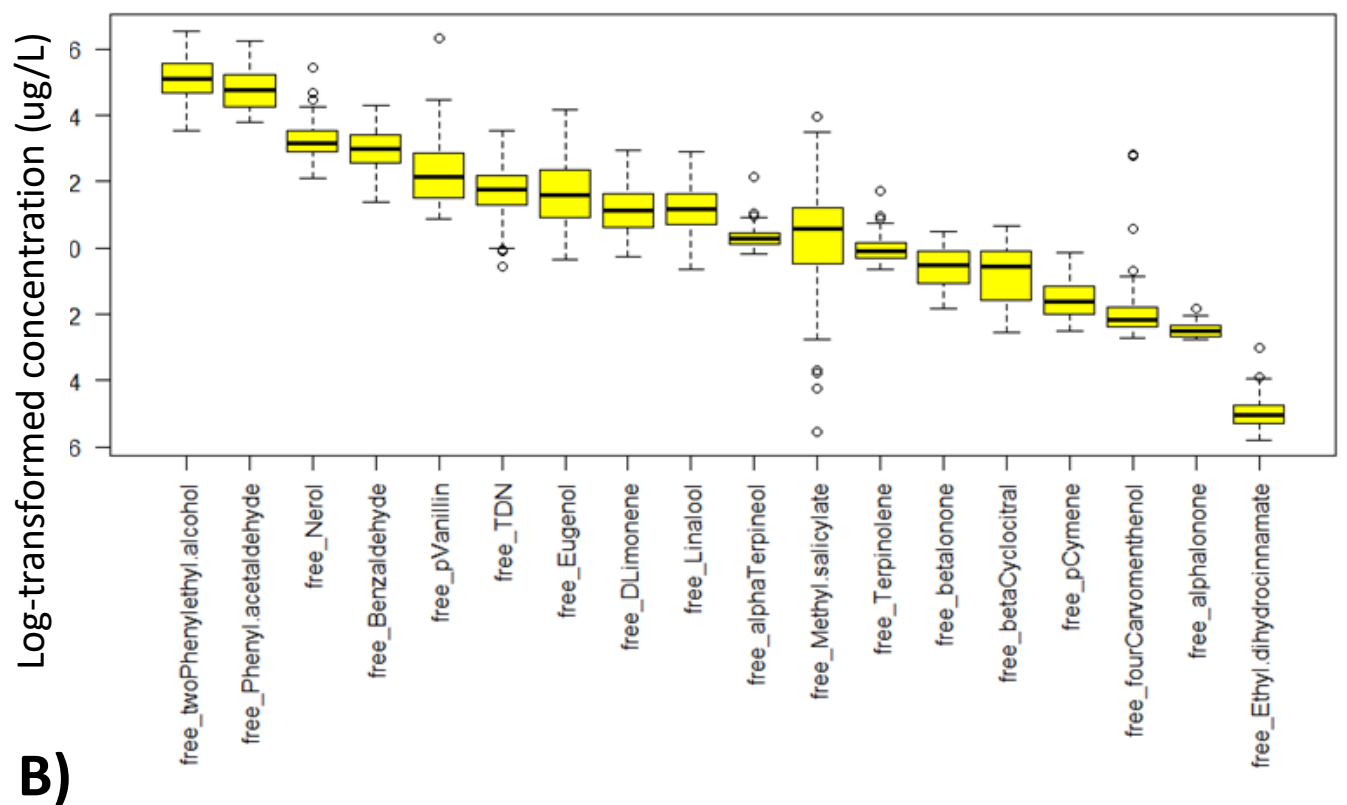

Total volatiles in $\mathrm{F} 1$ population

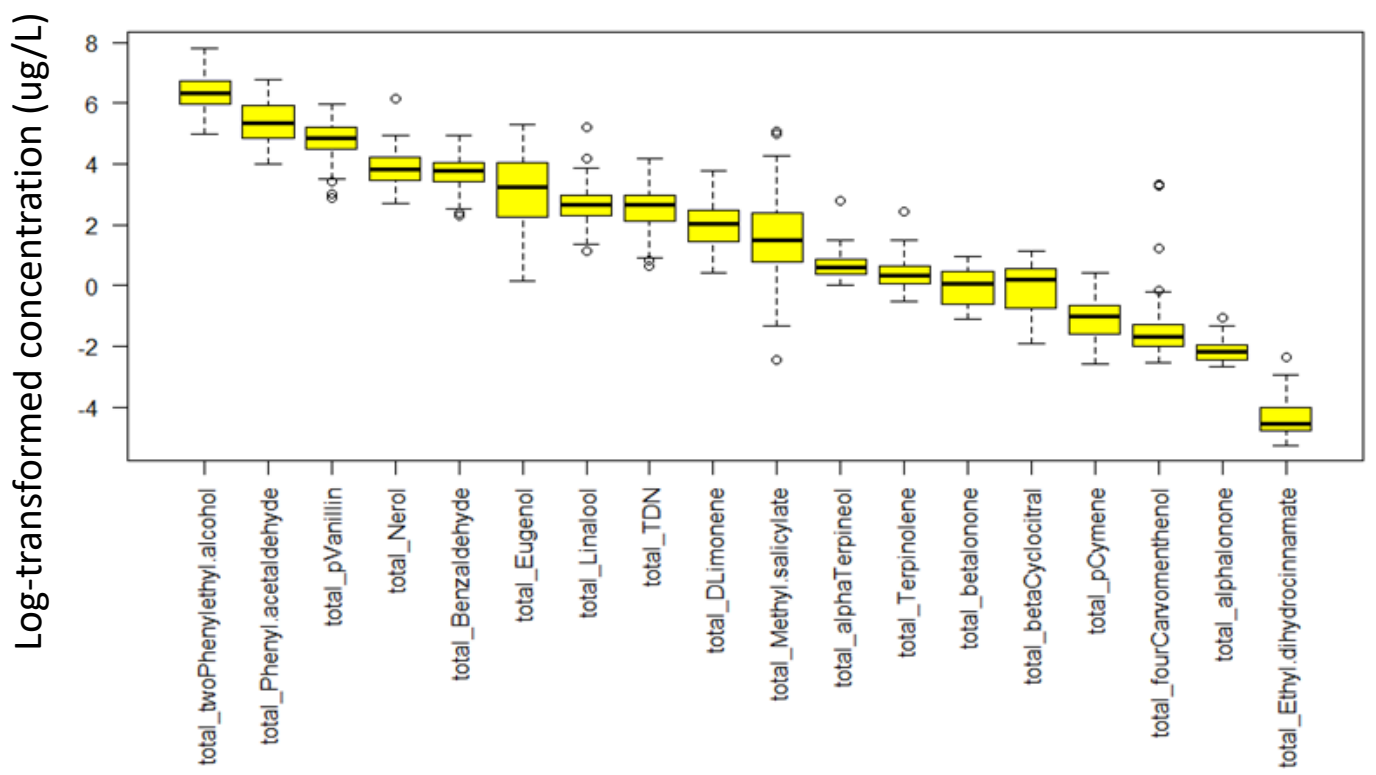

Figure 2.8: Boxplots demonstrating the variability of the (A) free and (B) total volatiles in F1 population cross from Norton and Cabernet Sauvignon (Cab). The concentrations of the volatiles in ug/L were log-transformed. 


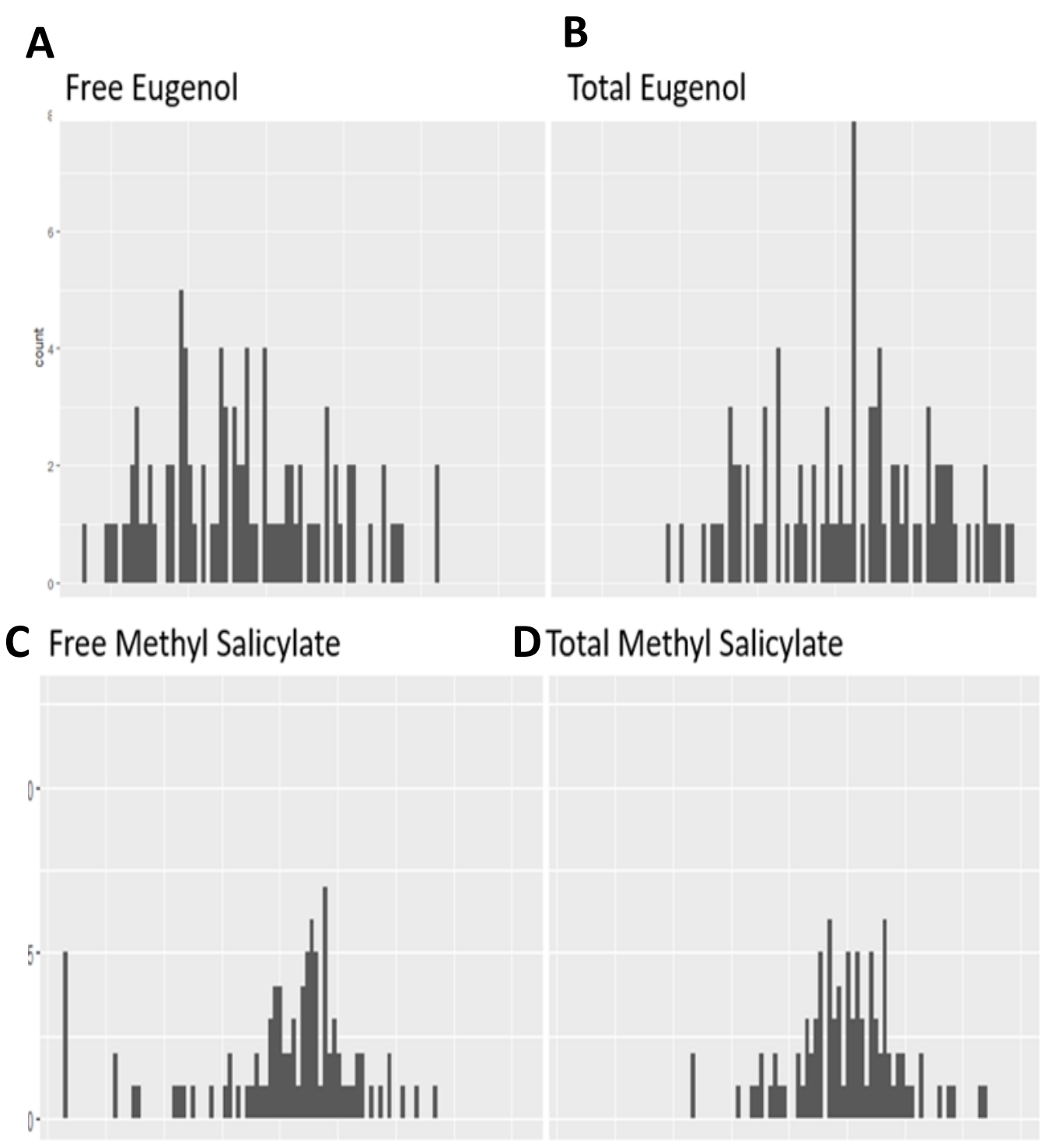

Figure 2.9: Frequency distribution of (A) Free Eugenol, (B) Total Eugenol, (C) Free methyl Salicylate and (D) Total methyl salicylate in F1 population of Norton and Cabernet Sauvignon. The concentration values in $\mathrm{ug} / \mathrm{L}$ were $\log$-transformed. 


\section{Tables:}

Table 2.1: Mean concentration (ug/L) and standard error of mean (SE. mean) of free volatiles, total volatiles and Wine volatiles in Norton and Cabernet Sauvignon.

\begin{tabular}{|c|c|c|c|c|c|c|c|c|c|c|c|c|c|}
\hline \multirow[b]{3}{*}{ Name } & \multirow{3}{*}{$\begin{array}{c}\text { Odor } \\
\text { threshol } \\
\mathrm{d}^{\mathrm{a}}\end{array}$} & \multicolumn{4}{|c|}{ Free Volatiles } & \multicolumn{4}{|c|}{ Total Volatiles } & \multicolumn{4}{|c|}{ Wine Volatiles } \\
\hline & & \multicolumn{2}{|c|}{ Norton } & \multicolumn{2}{|c|}{$\begin{array}{l}\text { Cabernet } \\
\text { Sauvignon }\end{array}$} & \multicolumn{2}{|c|}{ Norton } & \multicolumn{2}{|c|}{$\begin{array}{l}\text { Cabernet } \\
\text { Sauvignon }\end{array}$} & \multicolumn{2}{|c|}{ Norton } & \multicolumn{2}{|c|}{$\begin{array}{l}\text { Cabernet } \\
\text { Sauvignon }\end{array}$} \\
\hline & & Mean & SE.mean & Mean & SE.mean & Mean & SE.mean & Mean & SE.mean & Mean & SE.mean & $\begin{array}{c}\text { Mea } \\
n\end{array}$ & $\begin{array}{l}\text { SE.m } \\
\text { ean }\end{array}$ \\
\hline $\begin{array}{c}\beta- \\
\text { Caryphyllene }\end{array}$ & 64 & 0.14 & 0.06 & 0.07 & 0.00 & 0.14 & 0.02 & 0.08 & 0.01 & nd & nd & nd & nd \\
\hline p-Cymene & & 0.15 & 0.06 & 0.01 & 0.01 & 0.45 & 0.25 & 0.00 & 0.00 & 5.12 & 0.57 & 1.90 & 0.15 \\
\hline Terpinolene & $14 \mathrm{mg} / \mathrm{L}$ & 0.29 & 0.01 & 0.26 & 0.02 & 0.79 & 0.02 & 0.82 & 0.02 & 0.26 & 0.02 & 0.38 & 0.02 \\
\hline D-Limonene & 10 & 0.49 & 0.25 & 0.15 & 0.03 & 3.72 & 1.58 & 0.44 & 0.09 & nd & nd & nd & nd \\
\hline Methyl & & & & & & & & & & & & & \\
\hline Hexanoate & & 1.42 & 0.11 & 1.53 & 0.15 & 1.05 & 0.27 & 0.92 & 0.29 & nd & nd & nd & nd \\
\hline$\beta$-Ionone & 0.03 & 1.68 & 0.20 & 2.14 & 0.18 & 2.04 & 0.18 & 2.70 & 0.29 & nd & nd & nd & nd \\
\hline
\end{tabular}




\begin{tabular}{|c|c|c|c|c|c|c|c|c|c|c|c|c|c|}
\hline$\beta$-Cyclocitral & 0.15 & 1.73 & 0.22 & 1.79 & 0.13 & 1.17 & 0.18 & 1.17 & 0.13 & nd & nd & nd & nd \\
\hline$\beta$-Linalool & $6-25.2$ & 3.47 & 0.24 & 1.66 & 0.08 & 6.74 & 0.35 & 3.35 & 0.24 & 9.87 & 0.93 & 9.58 & 0.94 \\
\hline & & & & & & & & & & & & & \\
\hline Damascenone & 0.05 & 4.06 & 0.30 & 2.37 & 0.14 & 22.71 & 1.69 & 15.78 & 1.86 & 7.26 & 0.80 & 4.34 & 0.39 \\
\hline Ethyl & & & & & & & & & & & & & \\
\hline hexanoate & 14 & 4.60 & 0.87 & 4.87 & 1.25 & 2.53 & 0.54 & 4.42 & 1.95 & nd & nd & nd & nd \\
\hline TDN & 2.00 & 7.35 & 0.67 & 2.66 & 0.24 & 6.53 & 0.57 & 2.75 & 0.40 & 2.13 & 0.27 & 1.54 & 0.13 \\
\hline 1-Nonanol & 50 & 10.78 & 0.18 & 1.68 & 0.11 & 9.28 & 0.31 & 1.51 & 0.15 & 3.20 & 0.24 & 3.59 & 0.19 \\
\hline Nerol & 400 & 11.66 & 0.66 & 1.26 & 0.09 & 7.13 & 0.29 & 6.62 & 0.17 & nd & nd & nd & nd \\
\hline Methyl & & & & & & & & & & & & & \\
\hline Salicylate & 40.00 & 18.15 & 2.27 & 0.08 & 0.02 & 9.08 & 0.88 & 0.00 & 0.00 & 4.11 & 0.60 & 0.93 & 0.08 \\
\hline & & & & & & & & & & & & 15.6 & \\
\hline Benzaldehyde & 5000 & 27.43 & 2.62 & 15.58 & 2.14 & 19.54 & 3.40 & 9.03 & 3.11 & 31.99 & 2.87 & 2 & 1.64 \\
\hline Eugenol & 3.00 & 51.25 & 6.15 & 0.47 & 0.04 & 60.34 & 4.58 & 0.38 & 0.04 & nd & nd & nd & nd \\
\hline
\end{tabular}




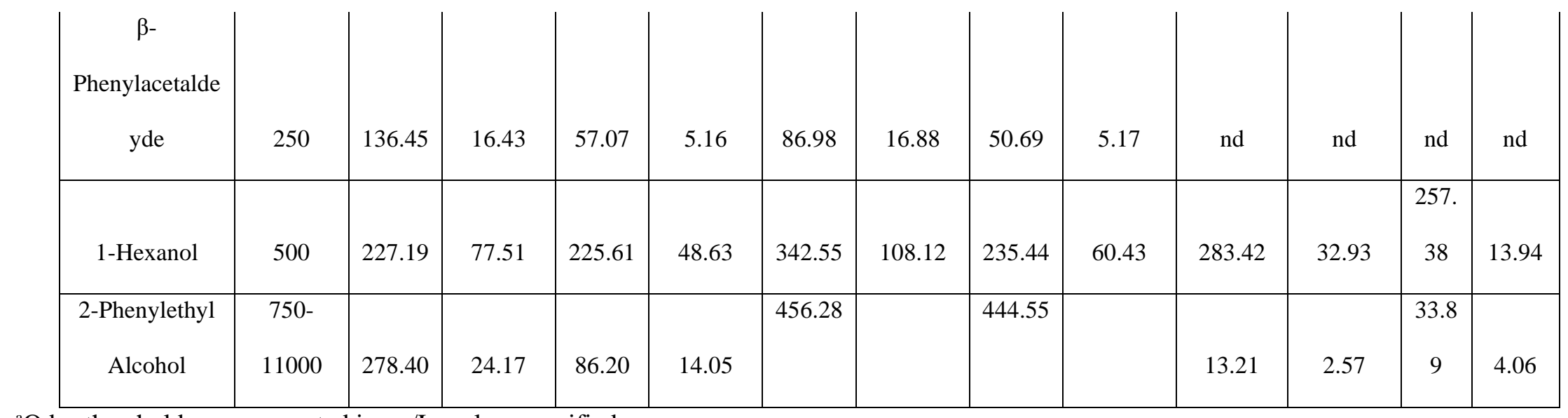

${ }^{a}$ Odor thresholds were reported in ug/L, unless specified. 
Table 2.2 Minimum, maximum and mean of concentration of free and bound volatiles in $\mathrm{ug} / \mathrm{L}$ in F1 population of Norton and Cabernet Sauvignon.

\begin{tabular}{|c|c|c|c|c|c|c|}
\hline \multirow{2}{*}{ Volatile compounds } & \multicolumn{5}{|c|}{ F1 population } \\
\cline { 2 - 7 } & \multicolumn{3}{|c}{ Free Volatiles } & \multicolumn{3}{c|}{ Total Volatiles } \\
\cline { 2 - 7 } & mean & $\min$ & $\max$ & mean & min & max \\
\hline 2-Phenylethyl Alcohol & 206.47 & 33.84 & 693.03 & 673.43 & 149.02 & 2453.36 \\
\hline \multirow{2}{*}{$\beta$-Phenyl Acetaldehyde } & 142.33 & 45.28 & 517.16 & 265.01 & 53.54 & 858.97 \\
\hline Nerol & 30.80 & 8.28 & 227.39 & 56.93 & 14.89 & 461.06 \\
\hline Benzaldehyde & 23.64 & 4.00 & 73.30 & 46.42 & 10.13 & 138.42 \\
\hline Eugenol & 9.65 & 0.72 & 65.59 & 42.10 & 1.17 & 200.85 \\
\hline TDN & 6.92 & 0.57 & 34.42 & 15.85 & 1.92 & 64.19 \\
\hline D-Limonene & 4.29 & 0.78 & 19.17 & 9.09 & 1.52 & 43.82 \\
\hline$\beta$-Linalool & 4.00 & 0.53 & 18.45 & 18.23 & 3.17 & 179.07 \\
\hline Methyl salicylate & 3.69 & 0.00 & 52.50 & 11.37 & 0.09 & 157.25 \\
\hline$\alpha$-Terpineol & 1.47 & 0.84 & 8.70 & 2.17 & 1.02 & 16.36 \\
\hline Terpinolene & 1.06 & 0.52 & 5.51 & 1.73 & 0.60 & 11.29 \\
\hline$\beta$-Ionone & 0.65 & 0.16 & 1.66 & 1.13 & 0.33 & 2.56 \\
\hline$\beta$-Cyclocitral & 0.61 & 0.08 & 1.93 & 1.21 & 0.15 & 3.10 \\
\hline p-Cymene & 0.27 & 0.08 & 0.87 & 0.41 & 0.07 & 1.51 \\
\hline
\end{tabular}




\title{
Chapter 3: Rootstock Modulation of Wine Aroma Compounds Under Varied Irrigation Regimes
}

Submitted to Journal of Agricultural Food Chemistry

\begin{abstract}
:
Characterization of aromas in understudied cultivars is challenging as important compounds may easily be missed if an inclusive initial selection of analytes of interest is not taken. A metabolomics-based approach followed by quantitation was utilized to study the subtle differences in aroma induced by rootstock and irrigation in a poorly studied variety. This study included cv. Chambourcin on three different commercial rootstocks (1103P, 3309C, and SO4) and own-rooted Chambourcin as well as all root-systems under varied irrigation regimes (full irrigation (full compensation of evapotranspiration (ET)), RDI (50\% of ET) and unirrigated. Wines were produced on a per vine basis over two years with volatiles analyzed using SPME-GC-MS/MS. Data were initially processed using an untargeted approach to identify spectral features ( $\mathrm{m} / \mathrm{z}$ by time) differences between treatments, followed by identification, confirmation, and finally quantification by authentic standards. We identified 877 and 682 features in 2017 and 2018 respectively, of which 382 and 221 respective features differed significantly among rootstocks. Of these features, 94 compounds were tentatively identified by library search and retention index, with 22 confirmed and quantified using standards. Own-rooted Chambourcin differed from other root-systems for multiple volatile compounds including Linalool, Ethyl Nonanoate, $\beta$-Damascenone, and TDN, with fewer differences among grafted vines. The average concentration of $\beta$-Damascenone present in own-rooted vines $(9.49 \mu \mathrm{g} / \mathrm{L})$ was significantly lower in other rootstocks $(8.59 \mu \mathrm{g} / \mathrm{L})$, whereas mean
\end{abstract}


Linalool was significantly higher in all rootstocks (mean of all $10.85 \mu \mathrm{g} / \mathrm{L}$ ) especially in 1103P $(12.5 \mu \mathrm{g} / \mathrm{L})$ compared to own-rooted $(7.6 \mu \mathrm{g} / \mathrm{L})$. Significant differences among irrigation regimes were also observed along with the interaction between rootstock and irrigation. $\beta$-Damascenone was higher in RDI $(9.03 \mu \mathrm{g} / \mathrm{L})$ than other treatments. Whereas Isoamyl acetate was significantly higher in fully irrigated 3309C $(1081 \mu \mathrm{g} / \mathrm{L})$ and RDI own-rooted vines contained the lowest concentration $(245 \mu \mathrm{g} / \mathrm{L})$. The approach outlined not only was shown useful for scientific investigation but may also be of interest for the industry. 


\section{Introduction:}

Many factors impact the final aroma and flavor of fermented beverages including the plant materials from which the beverage is made, yeast, and bacterial metabolism during the fermentation process and aging condition (Noble and Cole 2003). Volatile compounds present in grapes, as well as aroma precursors, are crucial for wine quality, with their concentrations modulated by a complex interplay between the natural environment (soil conditions, climate, temperature), vineyard management practices (irrigation, pruning, sun exposure) and vine genotypes, including the genotype of the rootstock (Asproudi et al. 2016; Ferreira and Lopez 2019; Francis and Newton 2005; Hjelmeland and Ebeler 2014; Parker et al. 2017). These grape-derived aroma compounds give the varietal character of the grapes, whereas the compounds formed due to yeast and bacterial metabolism during vinification as well as chemical transformations during vinification add complexity in the wine aroma (Fang and Qian 2006). To understand the complex wine aroma, and to decouple the effect of different factors on fruit and wine quality, targeted analysis of specific metabolites has been traditionally used (Balint and Reynolds 2017; Koundouras et al. 2009; Qian et al. 2009; Olarte Mantilla et al. 2018; Berdeja et al. 2014) where a few selected compounds are quantified. This practice can be suitable in vinifera cultivars where important odorants have been well characterized. However, in understudied interspecific hybrid cultivars where the important volatiles are not known, a more all-encompassing approach such as untargeted metabolomics is useful to avoid missing information buried in chromatograms.

About $80 \%$ of all Vitis vinifera grapevines planted are grafted to rootstocks that are derived from American Vitis species that confer resistance against phylloxera (Ollat et al. 
2014; Main et al. 2002). While planting own-rooted vines has the benefit of being cheaper and easier to maintain, this is only an option for regions where soils are immune to phylloxera infestation. Rootstocks also have been found to confer resistance to additional pests and pathogens such as nematodes (Ferris et al. 2012) and increased tolerance to abiotic stress such as drought (Serra et al. 2014; Zhang et al. 2016) and salinity. Rootstocks can also modify the scion by influencing the water and nutrient uptake (Tramontini et al. 2013), vigor (Jones et al. 2009a), canopy density, sun exposure, yield, thus impacting the fruit and wine quality in Vitis vinifera cultivars (Harbertson and Keller 2011; Keller et al. 2012; Olarte Mantilla et al. 2018; Vivian et al. 2018; Sun et al. 2011c; Jones et al. 2009a; Wang et al. 2019). By impacting water uptake, rootstocks can alter the plants' ability to tolerate water stress, and thus the interaction between different irrigation and rootstocks may also impact the volatile profile of grapes and wines as water stress has been known to impact the wine volatiles (Berdeja et al. 2014; Berdeja et al. 2015; Vivian et al. 2018; Koundouras et al. 2009). While interspecific hybrid cultivars are mostly grown own-rooted due to their innate phylloxera resistance, other potential benefits that grafting can confer on hybrid cultivars especially on wine aroma, as well as the performance of rootstocks on different irrigation regimes, has been poorly studied. To decouple the effect of rootstocks and irrigation factors on berry and wine quality in understudied hybrid cultivars where the important volatiles have not been known, the optimal approach to use is a more comprehensive untargeted metabolomics approach to extract useful information from chromatograms.

While the genotype of scion defines the fundamental fruit composition in the plants (Harbertson and Keller 2011), previous research has identified differences in fruit and 
wine compositional parameters, such as $\mathrm{pH}$, potassium $(\mathrm{K})$, anthocyanins and phenolic compounds between different rootstocks and own-rooted vines (Main et al. 2002; Jones et al. 2009a; Harbertson and Keller 2011; Koundouras et al. 2009; Nelson et al. 2015). In general, wines from own-rooted vines were found to have higher $\mathrm{pH}$ and total anthocyanins and tannins than wines from grafted vines. Nonetheless, certain rootstocks performed better in producing higher anthocyanin content than own-rooted vines among the five rootstocks and three scions studied. Though the differences were significant, they were minor (Harbertson and Keller 2011). Different rootstocks have also been shown to impact the concentration of volatile compounds with one study finding differences in volatile esters and 2,3-butanediols (Ough et al. 1968). Cabernet Sauvignon vines grafted to two different rootstocks and subjected to three different irrigation treatments also demonstrated differences in the total aroma potential of grapes estimated as phenol-free glycosyl-glucose (Koundouras et al. 2009). Similarly, rootstocks were found to have a positive effect on wine composition and quality in Shiraz vines grafted to three different rootstocks than own-rooted vines, where rootstocks increased important aroma volatiles including norsisoprenoids and higher alcohol compounds than wines from own-rooted vines (Olarte Mantilla et al. 2018). Similarly, rootstocks significantly affected the concentrations of many volatile compounds in wines from Monastrell grapes grafted to five different rootstocks and subjected to two irrigation treatments (Romero et al. 2019). High-vigor rootstocks, such as $1103 \mathrm{P}$, showed higher concentrations of alcohols, esters, and other volatiles than wines from lower vigor rootstocks (Romero et al. 2019). The impact of rootstocks on wine quality is attributed to an indirect effect on the scion, as genetic differences between rootstock cultivars can influence water availability to the 
scion, root dynamics, $\mathrm{N}$ uptake, canopy biomass and sun exposure (Stockert et al. 2013; Tramontini et al. 2013), which in turn can influence the microclimate of the fruit and impact the composition of wine volatiles (Young et al. 2016). While some research has been done to study the impact of various rootstocks on the fruit and wine composition of V. vinifera (Koundouras et al. 2009; Olarte Mantilla et al. 2018; Romero et al. 2019), the research of rootstocks on grape and wine aroma is scarce on the interspecific hybrid grapes and wines. This is likely because of the more limited planting of interspecific hybrids and the fact that their phylloxera tolerance generally allows them to be grown own rooted, though there are numerous other reason why grafting maybe beneficial (Thomas et al. 2017; Main et al. 2002).

In addition to rootstocks, water stress during the critical growth stages of a grapevine is known to cause negative effect on growth, reproduction, yield and thus on fruit and wine composition (Nelson et al. 2015; Vivian et al. 2018; Berdeja et al. 2014; Deluc et al. 2009). In an arid climate, irrigation resulted in wines with greater intensities of apple, citrus, and floral aromas and reduced earthy aroma in Chardonnay wines (Reynolds et al. 2007) and a better appreciation of wines from irrigated Chasselas vines (Vivian et al. 2018). Whereas excess irrigation resulted in wines with astringency and vegetal and black pepper aroma in Cabernet Sauvignon, compared to the more fruity and less vegetal character in wine from deficit irrigation (Chapman et al. 2005). Moderate water stress increased the concentrations of sugar, anthocyanins, some amino acids in Pinot noir grapevines grafted to two rootstocks (Berdeja et al. 2014). Under non-drought conditions, moderate water stress mediated through deficit irrigation leads to reduced water uptake and reduced shoot growth and yield leading to smaller berries with a higher 
concentration of total phenolic substances, anthocyanins and wine volatiles which are important for wine quality (Vivian et al. 2018; Romero et al. 2019). The improvement in grape quality is due to a reduction in vine vigor, mainly in the canopy density, that alters canopy microclimate in the fruit zone and increases the amount of light reaching the fruit (Bindon et al. 2007; Young et al. 2016; Vivian et al. 2018; Qian et al. 2009). Medium soil water deficit was found to correlate with positive wine sensory attributes (more fruity and less vegetal) with higher intensity of flavor and aroma attributes in deficit irrigation in Chardonnay grapes (Balint and Reynolds 2017). Limited water availability mediated through partial rootzone drying has also been reported to increase the concentration of grape aroma compounds and their precursors such as norisoprenoids compounds in Cabernet Sauvignon berries (Bindon et al. 2007). Similarly, wines from Merlot subjected to deficit irrigation contained a higher concentration of $\beta$-Damascenone, guiacol, 4methylguiacol than the well-watered vines (Qian et al. 2009).

Traditionally, the targeted analysis had been used to study the impact of rootstocks, irrigation and other viticultural management on the complex wine aroma and flavor, where few significant compounds or group of compounds were studied (Bindon et al. 2007; Berdeja et al. 2014; Koundouras et al. 2009; Romero et al. 2019). While targeted analysis has advantages of being simple, non-targeted metabolomics approach is comprehensive and can simultaneously detect numerous compounds (Schrimpe-Rutledge et al. 2016) and had been used in grapes and wine studies to understand grapevine berry development, fungal pathogen in juice, comparison between wild and vinifera volatiles, wine classification wine volatile, non-volatile and unspecified compounds that are potentially present in the wine (Cuadros-Inostroza et al. 2016; Flamini et al. 2014; 
Narduzzi et al. 2015; Roullier-Gall et al. 2014a; Schrimpe-Rutledge et al. 2016; Toffali et al. 2011; Schueuermann et al. 2019). This approach was also used to study rootstock modulation of grape and wine aroma in Shiraz grapes, where 152 compounds were identified in the chromatograms (Olarte Mantilla et al. 2018). While this type of analysis has value in identifying many unknown compounds, this approach has tremendous importance in uncharacterized and understudied cultivars when metabolites are not defined, as it can characterize complex general differences between populations. In untargeted approaches, spectral information from chromatograms is transformed into coordinates based on mass, retention time and associated signal amplitude, which are then aligned across all samples to detect features or putative compounds which are analyzed using statistical and multivariate analysis tools (De Vos et al. 2007; SchrimpeRutledge et al. 2016; Tautenhahn et al. 2012). Thus, this approach will be a better way to understand the volatile profile of hybrid grapevine Chambourcin and to unravel the impact of rootstocks and irrigation on its volatile profile which has hardly been metabolically studied. The study of the volatile profile of Vitis labrusca grapes and their hybrids have shown compounds such as methyl anthranilate and o-aminoacetophenone compounds, that cause the characteristic foxy taste of these grapes, which were not present in the vinifera grapes (Shure and Acree 1994). Studying only a few compounds might miss some of the compounds that were not present in vinifera but present in hybrid grapes.

Understanding the impact rootstocks have on water status is critical to maintaining the productivity and quality of grapevines in the changing climatic condition (Berdeja et al. 2014). With the expansion of grape growing in more unconventional areas, hybrid 
grapes due to their resistance to biotic and abiotic stress are grown as they offer important benefits that vinifera do not have (Reisch et al. 2012). It is essential therefore to study their grapes and wines aroma profile. Chambourcin is a French humidity-tolerant and phylloxera tolerant hybrid grape, derived from a cross between Seyve-Villard 12-417 with Chancellor (Thomas et al. 2017). Since hybrids such as Chambourcin offer important benefits vinifera does not have, it is imperative to better understand how they are impacted by complex environmental and rootstock interactions while planning future low input vineyards with great wine quality. In this study, Chambourcin vines were grafted to three different commercial rootstocks: 1103P, 3309C and SO4 and own-rooted and were subjected to three different irrigation regimes: $100 \%$ fulfillment of evapotranspiration (Full), 50\% fulfillment of evapotranspiration (RDI) and no irrigation. Wines from the experiment were analyzed using a metabolomics-based approach to understand the complex interplay of rootstocks and irrigation on wine volatiles.

\section{Materials and Methods:}

\subsection{Reagents and Chemicals:}

All aroma standards other than 1,1,6-trimethyl-1,2-dihydronapthalene (TDN) were purchased from Sigma-Aldrich (St. Louis, MO, USA) at $>98.8 \%$. TDN was synthesized and donated from Dr. Gavin Sacks lab at Cornell University (Kwasniewski, Vanden Heuvel et al. 2010). A C7-C30 hydrocarbon mixture, used for determination of Kovat's retention indices, was obtained from Sigma-Aldrich. Sodium chloride was purchased from Fisher Chemicals (Fair Lawn, NJ, USA). Ultrapure water (Type 1 water) 
was prepared using the ELGA Lab Water PURELAB Classic (High Wycombe, UK). LTartaric acid (99\%) was obtained from Sigma-Aldrich.

\subsection{Study design and sampling:}

The samples were collected from an experimental vineyard at The University of Missouri Southwest Center, Mount Vernon, Missouri in 2017 and 2018. The experimental vineyard was established in 2009 with Chambourcin scion grafted onto three different commercial rootstocks: 1103P, 3309C, S04 as well as non-grafted, ownrooted Chambourcin (('3309C'- V. riparia $x$ V. rupestris: '1103P'-V. berlandieri $x$ V. rupestris; 'SO4'- V. berlandieri $x V$. riparia). In addition to the four different root systems, three irrigation regimes were implemented in a full factorial design with irrigation treatments including Full irrigation, Reduced irrigation, and No irrigation (full replacement of evapotranspiration losses (ET), 50\% replacement of ET, and nonirrigated respectively). A total of 9 rows were used for this study. Each row had a different randomly assigned irrigation treatment and consists of 4, 4-vine rootstock blocks randomly ordered with two guard vines at either end of a study row. For this study, only the middle two vines (vine 2 and 3) were sampled from each block. During the establishment, all vines were fully irrigated until 2013 when the treatments began. Irrigation treatments began when water stress was observed, usually several weeks before veraison, as the site has ample spring precipitation. The fruit was harvested in 2017 and 2018 from the 71 plants ( 9 rows $* 4$ blocks $* 2$ vines) individually into separate bins. Vine 2 in row 10 reverted back to its $\mathrm{SO} 4$ rootstock, so this rootstock vine was not used in the 
analysis. More information about the vineyard design can be found at (Maimaitiyiming, Ghulam et al. 2017) and (Migicovsky, Harris et al. 2019).

\subsection{Winemaking:}

The wine was made on a per vine basis. Fruit from each vine was separately harvested and transported to the winery at the University of Missouri. The grapes were stored in a cold room at $4^{\circ} \mathrm{C}$ overnight and processed the day following harvest. The grapes were crushed and destemmed using Enoitalia destemmmer-crusher (Cerreto Guidi, Italy). Sulfur dioxide (50mg/L total) was added to each fermenter immediately after crush, with inoculation occurring approximately 12 hours later. The fermentations were carried out in 1-gallon fermentation vessels equipped with airlocks. Must was inoculated with GRE yeast (Lallemand, Petaluma, CA) at the rate of $1 \mathrm{~g} / \mathrm{L}$ in day 2 and rehydrated with Go-Ferm yeast nutrient (Lallemand) according to the manufacturer's rehydration protocol. The ferments were punched down twice a day for 10 days, at which point they were pressed. The wines were racked approximately 21 days after pressing. To decrease the headspace, marbles were added to containers as needed as well as any headspace purged with nitrogen gas. The wines were filtered and bottled in $355 \mathrm{ml}$ amber bottles. Following bottling all wines were stored at $4{ }^{\circ} \mathrm{C}$ until analysis.

\subsection{Extraction of wine volatiles:}

The extraction of wine volatiles was done using the same protocol as in Chapter 2, section 2.5 .

\subsection{HS-SPME-GC-MS and HS-SPME-GCMS/MS:}


In 2017 and 2018 analysis was conducted on wines using electron ionization in scan mode on a single quadrupole GC-MS or triple quadrupole (TQ), respectively. Semiquantitative analyses for both 2017 and 2018 were conducted using the triple quadrupole in a scan or using MRM mode for some compounds as needed. The HS-SPME GCMS conditions were similar to that in section 2.6 in Chapter 2. For GCMS/MS, the temperature of the transfer line was $240^{\circ} \mathrm{C}$, and nitrogen $(1.5 \mathrm{ml} / \mathrm{min})$ was used as the collision gas. The mass spectrometer was operated in electron ionization mode at $70 \mathrm{eV}$ with multiple reaction monitoring (MRM), with the monitored transitions. Data acquisition and qualitative analyses were performed using the MassHunter Workstation software version B.07.00 (Agilent Technologies).

\subsection{Data processing using untargeted metabolomics analysis:}

Data processing was done using the same protocol as in Chapter 2, section 2.7. Results were downloaded from the XCMS online on June 11-12, 2019. The extracted features (intensity of a given $\mathrm{m} / \mathrm{z}$ at a certain time) were used for further analysis in defining treatment differences. The features that are significant for rootstock or irrigation using ANOVA (FDR adjusted p-value $<0.05$ ) were filtered and used for further analysis. Principal component analysis (PCA) and partial least squares discriminant analysis (PLSDA) applied after unit variance (UV) were evaluated for sample discrimination.

\subsection{Identification and confirmation of the compounds:}

After identification of significant features using ANOVA, the compounds represented by the features were tentatively identified using the NIST MS Search v2.2, 
NIST 14 Mass Spectral Library database (Scientific Instrument Services, Ringoes, NJ, USA). Linear retention indices (RI) were calculated using Kovats' equation from a sequence of linear hydrocarbons from C7 to C30. Possible matches for volatile compounds were first identified by comparison of the mass spectral data within the NIST library and then verified as a valid prospect based on RI data.

The confirmation and quantitation of volatile compounds were achieved using calibration curves for each standard at five different concentration levels in cases where standards are available. For compounds whose standards were not available, semiquantitative analysis was done by assuming a response factor equal to one to 2-Octanol IS equivalents. Agilent MassHunter Quantitative Analysis (for QQQ) B.07.01 (Agilent Technologies) was used. Each standard was prepared in model wine solution $(8 \mathrm{~g} / \mathrm{L}$ of tartaric acid, dissolved in $13 \%$ ethanol solution (v/v), at $\mathrm{pH} 3.2$, adjusted with $\mathrm{NaOH}$ ). Due to a large number of features and potential compounds, a further level of filtering was needed to keep authentic standard costs to a reasonable level. Odor activity value was chosen as a way to ensure priority was given to compounds likely to impact wine quality rather than just metabolic shifts of unknown importance. Odor thresholds of compounds found in the literature were used to calculate their odor activity values which shows the relative contribution of each volatile compound to the final aroma of the wine.

\subsection{Statistical analysis:}

The data were analyzed using MetaboAnalyst 3.0 (Xia and Wishart 2016) and R statistical software (Team 2019). In MetaboAnalyst, the features (M(m/z) T(time in minutes)) with more than $50 \%$ missing values were removed, and remaining missing 
values were replaced by a small value (half of the minimum positive value in the original data). Data filtering using interquartile range was then performed to remove features arising from the baseline noises and to remove features that do not change throughout the treatments that are unlikely to be used for further analysis. Then sample normalization was performed to account for the differences among the sample, for the wine data, normalization was done using a reference sample which is the 2-Octanol internal standard area. Then, the data was log-transformed and auto-scaled (mean-centered and divided by the standard deviation of each variable) and then used for further statistical analysis. The autoscaling method was used to remove any variation comprised during analysis (such as a loss of instrumental sensitivity) of an original MS peaks list.

Univariate statistics such as one-way ANOVA followed by a posthoc test was performed for exploratory data analysis to identify the potentially significant features to discriminate the treatments under study. Multivariate principal component analysis (PCA) was performed with significant features for each year. PCA is especially useful in a data with a large number of correlated variables as it has the capacity to reduce the complexity of a huge dataset by extrapolating important information and displaying it as a set of new independent variables called principal components that collectively explain most of the variability in the original dataset. Using the first $20 \mathrm{PCs}$, we fitted a linear model with irrigation, rootstock, and irrigation and rootstock interaction to understand the effect of treatments on wine volatiles. The linear model was calculated using the ' $1 \mathrm{~m}$ ' function in $\mathrm{R}$ and the percentage of total variation explained by each $\mathrm{PC}$ was calculated using the ANOVA function, and only factors that explained a significant portion of variance $(\mathrm{p}<0.05)$ were visualized using ggplot 2 in $R$. 


\section{Results:}

To investigate the effects of subtle treatments (rootstock and irrigation) on wine metabolite concentrations, a comprehensive untargeted metabolomics approach was used to avoid missing analytes. A workflow of the metabolomics approach had been shown in Figure 3.1. More than 600 features were identified in both years and were then subjected to multivariate analysis. Subtle separation of treatments was observed through heatmaps and PCA analysis of wine features between own-rooted and different rootstocks, demonstrating that rootstock is modulating differences in the wine volatile metabolome (Figure 3.2 and 3.4). The significant features from ANOVA and PCA were used to tentatively identify 94 unique compounds by the NIST library (Figure 3.1), 24 of which were confirmed and quantified with authentic standards and calibration curves. The results from the quantitative data were in line with the results from the untargeted data showing the same subtle differences between treatments and significant differences between treatments for some important aroma compounds. The quantified data confirmed treatment impacts observed in untargeted results, but through proper calibration and minimization of the run to run variance with the use of an internal standard, significant differences of many important wine aroma compounds were observed.

\subsection{Untargeted Metabolomics Results:}

An initial untargeted screening of GCMS data of wine volatiles was conducted to identify metabolic features that define subtle differences Data processing using XCMS online identified a total of 682 and 877 metabolite features or putative compounds in 2017 and 2018 respectively (Figure 3.1). Analysis for each year was performed separately as the yearly difference was the major factor separating wine volatiles (results 
not shown). Among the features detected, 221 and 328 features were significantly different due to rootstocks regardless of the irrigation regimes (Figure 3.1, Supplemental Table S1 and S3) and 380 and 85 features causing significant differences between various irrigation regimes regardless of the differences in rootstocks in 2017 and 2018 respectively (Figure 3.1, Supplemental Table 2 and 4). The variability in wine metabolite features caused because of irrigation and rootstock in both years can be visualized using heatmaps (Figure 3.2A, 3.2B, 3.2C, and 3.2D), where the most significant top 25 features were shown. Both rootstocks and irrigation showed yearly differences in metabolite features. For example, in 2017, wine features were similarly expressed in RDI and None whereas in 2018, wine metabolite features were similar between Full and RDI (Figure 3.2A). Many features demonstrated contrasting expression for None and Full, a feature with higher intensity in None demonstrated the lowest intensity in the Full and vice versa in both years (Figure 3.2 A and B). RDI differentiated itself from both Full and None with reduced or very low intensity for many metabolites in 2017 but higher in 2018 . In 2017, the wine features due to full irrigation showed differential responses than none and RDI (Figure 3.2A). This indicated that vines under water stress (limited water availability) produced wine metabolite features that were different than those that are produced under water availability conditions. The overlapping between none and RDI suggested that vines experiencing water stress produced metabolites that are similar to each other irrespective of having different root systems. Another point to observe is that the RDI has a wider spread than none-irrigation treatment. In 2018, all the irrigation treatments overlap to some degree, however, RDI was more spread out than full and none. Although not a clear separation between rootstocks or irrigation, there were subtle 
differences between the rootstocks and irrigation treatments that drive differences in wine volatiles. This also indicated possible irrigation and rootstock interaction causing changes in the wine volatiles. Between rootstocks, 1103P and 3309C were similar to each other than SO4 and own rootstocks in both years (Figure 3.2C and 3.2D). Interestingly, ownrooted vines had the strongest differences of the root-systems, clearly contrasting them from all grafted vines, showing either very high or very low-intensity values of the identified features in both years (Figure 3.2 C and D). Among the three rootstocks used, it is interesting to observe that most of the intensity of the features of SO4was in between the own-rooted and other rootstocks (Figure 3.2 C and D). This indicated that the rootstocks have an impact on modulating features representing wine volatiles through own-rooted and grafted vines generally were the most different.

We also compared the features that were both common and different in years and among rootstock and irrigation. We found 45 significant features that were common in 2017 and 2018 between rootstocks as demonstrated by the Venn diagram (Figure 3.3A). Similarly, there were 170 and 16 features that were common between rootstocks and irrigation treatments in 2017 and 2018 respectively (Figure 3.3B and 3.3C).

\subsection{Multivariate analysis of wine features:}

To model the treatment entirety of the impact of the significant features as related to treatment, unsupervised principal components analysis (PCA) was performed. The impact of rootstocks on wine metabolic features can be observed in the PCA scores plot in which $64.7 \%$ and $10.2 \%$ of the total variance was explained by $\mathrm{PC} 1$ and PC2\% respectively in 2017 (Figure 3.4A) while in 2018, PC1 explained 37.6\% of the variance and mainly reflected separation between own-rooted Chambourcin and 1103P and PC2 
explained about $10 \%$ of the variance (Figure 3.4B). Although no clear distinction between groups was observed as shown in scores plot using PC1 and PC2 (Fig 3.4A and B), the highest separation was between own-rooted Chambourcin and all grafted vines. ANOVA using principal components followed by post hoc Tukey's test (data not shown) also supports the subtle differences between rootstocks whereas separation from ownrooted vines. Similarly, the distribution of wine volatile features due to irrigation treatment can be observed using PCA scores plot in which PC1 and PC2 explained 24.7\% and $14 \%$ of the total variance respectively in 2017 (Figure 3.4C) and 50.3\% and 13\% of the variation contributing the total of $63.3 \%$ of the total variation in 2018 (Fig 3.4D). Like in rootstocks, there was also no clear separation between the irrigation treatments in both years.

Using first 20 PCs in a linear regression model that included rootstock (own-rooted, 'SO4', '1103P' and '3309C'), irrigation (none, RDI, Full) and rootstock by irrigation interaction effect, we observed that the PC1, PC3, and PC16 contributed significantly up to $35 \%$ of variation due to rootstock in 2017 (Figure 3.5A). Irrigation had a significant effect on PC2, PC9, PC10, PC11, PC12, PC13, PC14, and PC20, contributing up to $20 \%$ of the variation in wine volatiles (Figure 3.5A). Similarly, in 2018, rootstocks contributed significantly to 5 PCs, explaining up to $35 \%$ of variation and irrigation contributed to 8 PCs explained significant variation up to 15\% of variation by irrigation in 2018 (Figure 3.5B). We also observed some significant rootstock by irrigation interaction impact in both years as explained by PC15 (contributed 15\% of variation) in 2017 (Figure 3.5A) and PC5, PC8, PC9, and PC12 (contributed up to 16\% of variation) in 2018 (Figure $3.5 \mathrm{~B})$. 


\subsection{Compound Identification and confirmation:}

To gain the most from metabolomics data, analytical signals (features) should be converted to metabolites or compounds to provide biological knowledge about the system. Identification of the compounds derived from the non-targeted analysis was done by comparing obtained mass spectra at a definite time with the NIST library as well as by comparing the calculated retention index with that of literature.

We identified 94 different compounds in wine 2017 and wine 2018 from the features that are significantly different due to rootstocks and irrigation among which 76 compounds were identified in 2017, whereas we identified 45 compounds in wine 2018 (Supplemental Table S5). In 2017, 26 compounds were found to be significantly different due to both rootstocks as well as irrigation whereas, 33 compounds are significantly different only due to irrigation in wine 2017 and 10 compounds are significantly different in wine 2017 due to rootstock effect only. In 2018, 28 compounds were found to be significantly different in wine due to rootstock effect, whereas, irrigation caused 18 compounds to be significantly different and 3 compounds were found to be similar in both rootstock and irrigation effect in wine 2018. Compounds cannot be identified for many features as positive relation of features to compound spectra in the database was not possible for many features due to limitations of the database, extraneous signals associated with solid-phase microextraction, potential co-elution causing a combined spectra or poor spectral differentiation within certain common classes of compounds (e.g. monoterpenes). Additionally, in some cases, identification was impossible due to the lack of available authentic standards. 


\subsection{Quantitation of the compounds:}

To further confirm and quantify the compounds identified from the significant features (non-targeted analysis), we used authentic standards and calibration curve and then analyzed using ANOVA for each compound using both treatments and interaction as factors to make sure if the identified compounds were causing differences due to rootstocks as well as irrigation treatments. Among 94 compounds identified in wines in 2017 and 2018 that are different due to rootstocks and irrigation, a subset of 21 and 22 compounds were quantified in 2017 and 2018 respectively, considering the odor activity of the compounds and cost of the compounds. Some of the compounds cannot be confirmed due to the lack of authentic standards.

We performed ANOVA for the above metabolites sets and found that the rootstock and irrigation interaction effect was significant for both year 2017 and 2018 for several compounds (Table 3.1). As shown in the table, 12 compounds showed significant rootstock and irrigation interaction effect in 2017, whereas, only one compound has a significant interaction effect between rootstock and irrigation in 2018 (Table 3.1). We found significant rootstock and irrigation interaction effect in volatiles including Isoamyl acetate, Terpinolene, Ethyl heptanoate, Ethyl decanoate, 1-Nonanol, Ethyl dihydrocinnamate, Ethyl myristate, Ethyl palmitate, 1-Dodecanol, and Isoamyl hexanoate in 2017 while Linalool is the only compound that showed significant rootstock and irrigation interaction effect in 2018 (Table 3.1). The compounds which are also significant in rootstock and irrigation main effect are shown in (Table 3.2). Important wine volatiles such as $\beta$-Damascenone, Methyl Octanoate, Ethyl Nonanoate, and Linalool $(p$-value $=0.06)$ was significantly different between rootstocks in 2017. In 2018, the wine 
volatiles that showed a significant difference between rootstocks at $5 \%$ level of significance are $\beta$-Damascenone, Methyl Octanoate, Ethyl Heptanoate, Ethyl octanoate, Ethyl Nonanoate, 1-Octanol, 1-Nonanol, TDN, Ethyl myristate, Ethyl palmitate, Isoamyl hexanoate, and 2-Phenylethyl acetate

Compounds that are also found to be significantly different from irrigation treatments in 2017 are $\beta$-Damascenone, Terpinolene, and Linalool while Terpinolene, Methyl octanoate, 1-Octanol, 1-Dodecanol, Biphenyl, and Ethyl palmitate are significant in 2018. RDI resulted in a higher concentration of compounds including $\beta$-Damascenone, Terpinolene, 2-Octanone, linalool, 1-octanol, ethyl dihydrocinnamate, ethyl myristate, ethyl palmitate, isoamyl hexanoate and 2-phenylethyl acetate in 2017 but not in 2018. In 2018 , None irrigation led to an increase in the concentration of compounds including $\beta$ Damascenone, Isoamyl acetate, Methyl octanoate, Ethyl heptanoate, Ethyl octanoate, Ethyl nonanoate, 1-octanol, Ethyl decanoate, 1-Nonanol, TDN, Ethyl dihydrocinnamate, Isoamyl hexanoate and 2-Phenylethyl acetate (Table 3.2).

Generally, grafting Chambourcin vine to different rootstocks caused either an increase or decrease volatile compounds in wines in both years. Linalool and Ethyl nonanoate were found to be higher in concentration in wines in rootstocks than ownrooted Chambourcin wines in 2018 (Figure 3.6) while wines from own-rooted vines showed higher concentration for $\beta$-Damascenone and TDN in both 2017 and 2018 (Figure 3.7). 


\section{Discussion:}

Vine water status and root-system choice have been demonstrated to have an important impact in a wide array of wine chemistry, including factors important to flavor and quality (Berdeja et al. 2014; Harbertson and Keller 2011; Romero et al. 2019). To understand the complex interplay of various factors during grape development that impact the grape and must composition and thus wine composition, various metabolic approaches have been used. Targeted analysis of volatiles has been the standard for decades as it allows quantification of selected few numbers of compounds which in turn can be related to odor thresholds and theoretically explain treatment impacts on aroma (Bindon et al. 2007; Callejon et al. 2012; Canuti et al. 2009; Wang et al. 2019; Berdeja et al. 2014; Fang and Qian 2006; Wooldridge et al. 2010). This however assumes that all compounds of importance have been identified, as well as that odor thresholds are accurate (and immobile). While this is more likely to be accurate in well studied $V$. vinifera cultivars such as cv. Cabernet Sauvignon or cv. Pinot noir, interspecific hybrids such as cv. Chambourcin has had far fewer studies conducted. As such, it is appropriate to take a more inclusive initial analysis approach to avoid missing important chemical differences induced by the treatments, before deciding what compounds are of interest. This approach stems from metabolomics, which attempts to widen the narrow focus of analysis out so far as to include all metabolites, or at least those with certain properties. This approach has proved invaluable in situations where stark differences are expected such as comparing mutant and wild type populations, infected and healthy individuals (Schueuermann et al. 2019). This type of analysis however is far less used when attempting to characterize subtle differences that are inherent in a biological system. 
When dealing with a genetically identical grapevine scion (cv. Chambourcin (in this case) grafted to genetically different root-systems with three different irrigation, a metabolomics-based approach was used to characterize the subtle differences caused due to rootstocks and irrigation on the Chambourcin wine volatiles (Fig 3.6).

More than 800 features or putative compounds were detected in the wines from the grafted and own-rooted vines due to treatments in both years which is a common acceptable number of features in metabolomics studies (Teh et al. 2019; Schueuermann et al. 2019). The number of features was narrowed to identify the most important features that contribute the most to the differences between treatments using various unsupervised and supervised analyses. To get a meaningful interpretation from the features in terms of biological functionality as well as to understand the flavor threshold, quantification of compounds is important. It is interesting to note that 94 compounds were identified from those features from our study which compares favorably with 99 compounds detected in Pinot noir due to barrel storage (Schueuermann et al. 2016) and 175 features due to ripening differences in Shiraz grapes (Šuklje et al. 2019) and 115 metabolites due to berry development in Cabernet Sauvignon and Merlot grapes (Cuadros-Inostroza et al. 2016).

Compounds cannot be identified for many features as positive relation of features to compound spectra in the database was not possible for many features due to limitations of the database, extraneous signals associated with solid-phase microextraction, potential co-elution causing a combined spectra or poor spectral differentiation within certain common classes of compounds (e.g. monoterpenes). Additionally, in some cases, 
identification was impossible due to the lack of available authentic standards. Among the identified compounds, 22 were quantified in both years in our study among which Isoamyl acetate, $\beta$-Damascenone, Linalool, Ethyl dihyrdocinnamate, Ethyl heptanoate, and Ethyl octanoate were present above their odor threshold (Table S2). For example, $\beta$ Damascenone whose odor threshold is $0.05 \mathrm{ug} / \mathrm{L}$, is present well above its odor threshold in wines with an average concentration of $8.82 \mathrm{ug} / \mathrm{L}$.

Climatic conditions change with each year, resulting in differences in the chemical composition or metabolites of the grapes, which ultimately result in differences in wine volatile composition (Arapitsas et al. 2020; Lee et al. 2009; Schueuermann et al. 2016). Since metabolites are the intermediates and end products of cellular regulatory processes, their levels can be regarded as the ultimate response of biological systems to environmental change. Vintage or yearly variation is a strong influence in wine flavor in our study (climatic data not shown), therefore wine volatiles were analyzed separately to be able to observe the subtle impact due to treatments. 45 metabolic features were found to be common in both years. Some metabolites are significant in both years due to rootstocks and irrigation, whereas some of the metabolites are significant only in one year and not in another year. For example, $\beta$-Damascenone is significantly different between different irrigation in 2017, whereas in 2018, there are no significant differences between irrigation treatment (Table 3.2). Similarly, isoamyl acetate is significantly different between different rootstock treatments in 2017, but not in 2018 (Table 3.2). In a recent study, the wine volatile composition was found to be significantly different between years due to climatological differences in two growing seasons, in which one year was 
drier and warmer and the other year was wet (Olarte Mantilla et al. 2018) especially, the own-rooted shiraz contained a higher concentration of acetate esters than rootstocks vines and were associated with higher vigor. Also, the study found that $\beta$-Damascenone is lower in its own-rooted vines that have high vigor due to a decrease in sunlight exposure.

The wines from own-rooted Chambourcin vines were found to be different from the wines from grafted Chambourcin in both 2017 and 2018 as shown from the PCA scores plot. There is not a complete separation between rootstocks or irrigation treatments in both years. The rootstocks overlapped with each other demonstrating subtle differences between them and a clearer separation from own-rooted wines. This partial separation in the PCA scores plot could indicate that there are distinct patterns in at least some metabolite levels that distinguish between the groups, however, the patterns may be weak, or confounded by strong variations in metabolites due to other factors associated with individual variabilities. Since the same cultivar, Chambourcin was grafted to different rootstocks, a huge amount of similarity in the system is expected. Such subtle but significant impact had also been observed between rootstocks grafted to the same scion for many fruit composition and quality traits, however, more pronounced differences between different scion cultivars grafted to the same rootstock were observed (Harbertson and Keller 2011), which reiterates the need for a careful investigation as they are the same system. One important aspect to consider is that minute differences are remarkable in terms of wine economics as wine is appreciated for its subtle differences due to terroir and vintage even though made from the grapes with identical genetic architecture (Bisson et al. 2002a). 
Another factor causing significant differences between wines from grafted and own-rooted wines was the differences in the genetics of the rootstocks. The use of genetically different rootstocks derived from crosses between three Vitis species $V$. rupestris, $V$. riparia and $V$. berlandieri for the specific adaptation to specific environmental conditions might have a direct or indirect influence on many physiological processes of the grapevine that ultimately impact the aroma development in grapes and in turn modulate the aroma differences in the wines. Rootstocks are known to affect numerous vegetative and reproductive parameters of the scion such as water and gas exchange, canopy growth, yield, berry basic chemistry, and berry phenolics, and anthocyanins (Koundouras et al. 2009; Olarte Mantilla et al. 2018). This explains why the rootstocks are different from the own-rooted. However, the subtle differences that are constantly manifested between different rootstocks might be due to the similarity of one parent or the other (similar to half-sib) in all three of the rootstocks that might have contributed to many similarities. For example, $1103 \mathrm{P}$ and $3309 \mathrm{C}$ both have $V$. rupestris as the common parent contributing $50 \%$ genetically.

There was also a subtle impact on wine metabolites observed due to irrigation impact. The impact of irrigation was more pronounced in wine 2017 as there was some sort of separation between full and RDI (Figure 3.2A). Although total annual precipitation received in 2017 was higher than 2018 (data not shown), 2017 received only four significant precipitation greater than 0.5 inches whereas 2018 received eight such significant precipitations which might have contributed to lower irrigation impact in 2018. Climatic conditions change with each year, resulting in differences in the chemical composition or metabolites of the grapes, which ultimately result in differences in wine 
volatile composition. Irrigation effect post veraison also caused a significant effect on berry secondary metabolites (Koundouras et al. 2009). Changes in wine metabolites were observed due to irrigation in both years. In 2017, the wine features due to full irrigation demonstrated differential responses than none and RDI. This indicated that vines under water stress (limited water availability) produced wine metabolite features that were different than those that are produced under water availability conditions. The overlapping between none and RDI suggested that vines experiencing water stress produced metabolites that are similar to each other irrespective of rootstocks. Another point to observe is that the RDI had a wider spread than none-irrigation treatment. Irrigation has been an important factor to manipulate aroma content in grapes as severe water stress during anthesis to harvest can negatively impact the growth and development of plants including grapes. Deficit irrigation increased volatile content in apples in less vigorous rootstock whereas decreased volatile content in high vigorous rootstock (Bianco et al. 2008). In grapes, reduced irrigation also increased the aroma concentrations in fruit along with sugars and amino acids (El-Ansary and Okamoto 2007). Mild water stress was found to benefit Sauvignon blanc grapes by increasing their aroma potential, whereas severe water stress has the opposite effect of decreased aroma potential (des Gachons et al. 2005). Vigorous rootstocks due to their ability to extract soil water by extracting larger soil volumes, compared to less vigorous trees can reduce the intensity of root-generated hormonal signals. Water deficit activates the hydraulic and chemical signals (such as $\mathrm{ABA}$ ) from the drying roots to the shoots that subsequently lead to reduced water use through decreased stomatal conductance through stomata closure. This leads to growth inhibition through decreased photosynthetic activity and impact on the quality of the 
fruit. Stomatal regulation of leaf gas exchange plays an important role in plant response to water stress. Deluc et al. (2009) using metabolomics and transcriptomics found that water deficit affected the ABA metabolic pathway in Cabernet Sauvignon and Chardonnay, with a high abundance of 9-cis-epoxycarotenoid dioxygenase (NCEDI) transcripts. The metabolic responses of grapes to water deficit varied with the cultivar, showing differences in $\mathrm{ABA}$, isoprenoid, carotenoid, amino acid, and fatty acid metabolism. Berdeja et al. (2014) found the effect of two rootstocks and irrigation treatment and their interaction on Pinot noir fruit metabolites but did not explore the effect on wine volatiles. There was a distinct separation of metabolites according to phenological stages, however, no clear separation was observed between different rootstocks.

Quantitation of the significant compounds based on the results from untargeted metabolomics analysis also demonstrated differences due to rootstocks and irrigation. We observed significant differences between own-rooted Chambourcin and Chambourcin grafted to rootstocks in many volatiles which indicated grafting is causing significant changes to the scion (Table 3.1). Such differences in volatile profiles of wines between own-rooted and rootstocks were also been observed in Shiraz (Olarte Mantilla et al. 2018) and Monastrell grapes (Romero et al. 2019). Monastrell grapes grafted to different rootstocks and treated with deficit irrigation showed significant differences in aroma profile between rootstocks and irrigation. Being a vigorous rootstock, 1103P wines showed a higher concentration of aromatic compounds including alcohols, esters, and acetic acid than wines from other rootstocks. However, in that study, own-rooted Monastrell grapes were not included in the study. No significant interaction between 
rootstock and irrigation were observed. (Wang et al. 2019) found a higher concentration of total esters on own-rooted vines than on grafted vines in Cabernet Sauvignon.

The quantitative (semi-quantitative) data from 2017 and 2018 differed from each other in concentrations (Table S2). This might be due to differences in growing conditions and environmental factors in both years that can significantly impact the aroma profile of grapes and wines. However, the wines from 2017 were analyzed after two years after bottling, whereas, wines from 2018 were analyzed just after bottling. Aging is known to increase or decrease certain compounds due to various reactions that take place in wine (Liu et al. 2016). The concentrations of acetate esters, ethyl esters, norisoprenoids, and terpenes were found to be lower in 2017 wines, whereas, the higher chain fatty acids esters were found to be higher in 2017 wines due to aging. It has been found that the concentration of ethyl esters decreases over time due to hydrolysis (Liu et al. 2016).

Analysis of the quantitative data of 2017 and 2018, showed a significant increase in concentrations of isoamyl acetate in wines regardless of treatments (Table 3.1). Fermentation produces many compounds that are not seen in berries, volatiles that is not in berries but present in wine that is created from berry precursors. Isoamyl acetate is an important ester derived during fermentation by Saccharomyces cerevisiae (Wyk, 1975). In 2017, Chambourcin wines regardless of all treatments, isoamyl acetate is present at a concentration of $495 \mathrm{ug} / \mathrm{L}$, which is 16.5 times higher than the odor threshold, thereby, showing the significance of this compound in the aroma of Chambourcin wines. This concentration was found to decrease in 2018 with an average concentration of 298.5 $\mathrm{ug} / \mathrm{L}$, which is about 10 -fold higher than the odor threshold. Isoamyl acetate was found to 
be the compound responsible for the characteristic fermentation bouquet in Pinotage wine (avg concentration $15.6 \mathrm{mg} / \mathrm{L}$ ) and found to vary significantly from one vintage to another (Van Wyk et al. 1979). The synthesis of isoamyl acetate by S. cerevisiae during fermentation of wine has been found to be due to the activity of isoamyl alcohol acetyltransferases (Plata et al. 2003).

Of the wines prepared from all the grafted vines, the $\mathrm{SO} 4$ grafted Chambourcin had the lowest level of isoamyl acetate, 2-octanoate, linalool, 2-phenylethyl acetate and 1dodecanol in both years, while, the concentration of volatile ethyl esters such as methyl octanoate, ethyl heptanoate, ethyl octanoate, and ethyl decanoate were higher in the wines from $\mathrm{SO} 4$ rootstocks. Volatile esters are fermentation-derived products and play a key role in the sensorial quality of the wine, producing fruity, candy and perfume-like aroma at low concentrations, while masking the other varietal aromas and yielding synthetic fruit or fruit candy aroma to the wine at higher concentrations. Acetate esters and ethyl esters are known to be formed from the same pathway, but different precursors.

$\beta$-Damascenone was found higher in vines that are own-rooted (mean of 9.49 $\mathrm{ug} / \mathrm{L}$ ) than grafted vines (mean of $8.59 \mathrm{ug} / \mathrm{L}$ ) in both years (Table S2). $\beta$-Damascenone is a C13-norisoprenoid formed from the degradation of carotenoids and is known to increase with shading. The decrease in vigor using low to medium vigor rootstocks (1103P, 3309C, and SO4) might have contributed to the lower concentration of this compound in vines grafted with rootstocks than the high-vigor own-rooted Chambourcin (Figure 3.7). Studies have also found an increase in $\beta$-Damascenone with water stress, which is consistent with the results of higher $\beta$-Damascenone concentration in wines from vines treated with RDI and None. The presence of this compound in all wines above 
the threshold (4-5 ug/L in wine matrix) demonstrated that this compound is a key aroma component in Chambourcin wines. Although this compound has honey, rose, baked apple aroma, it has been known to impact the wine aroma by interacting with other aroma components.

Ethyl heptanoate and ethyl octanoate are fermentation-derived ethyl esters that are present above the odor threshold in Chambourcin wines in both 2017 and 2018 (Table S3). These compounds are present in higher concentrations in wines from own-rooted vines. Unirrigated vines also had a higher concentration of these compounds (Table S2). Wines made from vines under deficit irrigation were associated with descriptors like fruity and less vegetal characters. This might be due to the activation of alternative biosynthetic pathways that leads to greater carbon flux that increases amino acids (precursors of esters in wines) and in carotenoids, resulting in more fruity aromas (Balint and Reynolds 2017). Linalool is a monoterpene derived from the mevalonate pathway with a flowery aroma characteristic of Muscat grapes. Wines from vines grafted to $1103 \mathrm{P}$ had a higher concentration of linalool (Table 3.2), above its odor threshold. Vines under deficit irrigation had a higher concentration of linalool in the wines. Own-grafted Chambourcin produced wines with the lowest level of linalool.

Many studies have found that grafting impacts both the aboveground and belowground functioning of the plant and the impact of this relationship is due more to the rootstock-scion interaction than to the individual effect of the rootstock or the scion (Warschefsky et al. 2016). The genotype of rootstock and scion plays a vital role in the phenotypic variation of a plant; however, many other factors impact rootstock scion interactions including the age of the grafted individuals, the grafting technique employed, 
seasonality, the age of the graft union, genotype by environment and genotype (scion) by genotype (rootstock) by environment interactions, root morphology and architecture, rootstock scion compatibility, and root-microbe interactions. Besides volatiles, rootstocks impart a significant impact on the sugar content, phenolic compounds, including anthocyanins, and the antioxidant capacity in vinifera grapes (Cheng, Wei et al. 2017). Such factors can have a major impact on the wine aroma or in some cases, small differences that may contribute to some changes. However, many such factors may collectively impact the wine aroma. In our study designed to examine the impact of such two factors (irrigation and rootstocks), we observed significant rootstock irrigation impacts on both years, with 2017 having a more pronounced effect on many important volatiles. For example, isoamyl acetate was found significantly higher in wines from fully irrigated 3309C vines, whereas the lowest concentration was found in own-rooted RDI vines. Similarly,1-octanol, isoamyl hexanoate, ethyl dihydrocinnamate, and 2phenylethyl acetate are found significantly higher in 1103P vines RDI treated vines, whereas ethyl tetradecanoate, ethyl hexadecanoate, and 2-octanone are found significantly higher in RDI 3309C vines. The interaction effect shows that the relationship of wine aroma with the factors is not simple, but complex interactions between various processes inside that plant that ultimately impact the final wine product. This water limitation and rootstock genotype interaction might alter various berry metabolic pathways related to berry composition through transcriptomic pathways (Berdeja et al. 2014; Berdeja et al. 2015). Isoamyl acetate was found significantly higher in wines from fully irrigated 3309C vines, whereas the lowest concentration was found in own-rooted RDI vines. Similarly, Biphenyl was found in the highest concentration in 
own-rooted unirrigated vines. one-octanol, isoamyl hexanoate, ethyl dihydrocinnamate, and 2-phenylethyl acetate are found significantly higher in 1103P vines RDI treated vines, whereas ethyl tetradecanoate, ethyl hexadecanoate, and 2-octanone are found significantly higher in RDI 3309C vines.

\section{Conclusion:}

Using a metabolomics-based approach, we were able to identify subtle differences between rootstocks and irrigation in a hybrid cultivar Chambourcin grafted to three different rootstocks and own-rooted. Because of its inclusive nature, this approach provides advantages over the traditional targeted approach especially in understudied cultivar such as Chambourcin where there are limited studies regarding the volatile profile. We identified around 800 metabolite features and using multivariate approaches, identified 94 unique compounds. We confirmed and quantified 24 compounds in 2017 and 2018. In 2017, 12 compounds showed significant rootstock and irrigation interaction effect, whereas, only one compound has a significant interaction effect between rootstock and irrigation in 2018.

Rootstocks caused either increase or decrease of volatile compounds in wines in both years. Linalool and Ethyl nonanoate were found to be higher in concentration in wines in rootstocks than own-rooted Chambourcin while wines from own-rooted vines showed the highest concentration for $\beta$-Damascenone, Methyl octanoate, Ethyl heptanoate, ethyl octanoate, ethyl decanoate, TDN. In irrigation, RDI resulted in a higher concentration of compounds including $\beta$-Damascenone, Terpinolene, 2-Octanone, linalool, 1-octanol, 
ethyl dihydrocinnamate, ethyl myristate, ethyl palmitate, isoamyl hexanoate and 2phenylethyl acetate in 2017 but not in 2018 .

The analysis done in this chapter is important as this will pave the pathway to analyze berry volatiles in Chapter 4. One of the important directions towards the future work related to this project will be to correlate wine and berry metabolites and to see the potential of predictability of wine metabolites based on berry metabolites. Since we have collected multiple phenotypic data in the same vines at the same time, integration of berry and wine volatiles with other phenotypes such as ionomics, RNA seq, remote sensing, and physiology will be very interesting to evaluate the physiological and biochemical changes in vines that will ultimately change the wine metabolites esp. aromas and flavors. Understanding the impact of rootstocks and irrigation on wine volatiles will prove useful in developing viticulture practices to manipulate grapes aroma to produce wine with desired aroma quality. 


\section{References:}

Arapitsas P, Ugliano M, Marangon M, Piombino P, Rolle L, Gerbi V, Versari A, Mattivi F (2020) Use of Untargeted Liquid Chromatography-Mass Spectrometry Metabolome To Discriminate Italian Monovarietal Red Wines, Produced in Their Different Terroirs. Journal of Agricultural and Food Chemistry. doi:10.1021/acs.jafc.0c00879

Asproudi A, Petrozziello M, Cavalletto S, Guidoni S (2016) Grape aroma precursors in cv. Nebbiolo as affected by vine microclimate. Food chemistry 211:947-956

Balint G, Reynolds AG (2017) Irrigation level and time of imposition impact vine physiology, yield components, fruit composition and wine quality of Ontario Chardonnay. Scientia horticulturae 214:252-272

Berdeja M, Hilbert G, Dai ZW, Lafontaine M, Stoll M, Schultz HR, Delrot S (2014) Effect of water stress and rootstock genotype on Pinot Noir berry composition. Australian Journal of Grape and Wine Research 20 (3):409-421. doi:10.1111/ajgw.12091

Berdeja M, Nicolas P, Kappel C, Dai ZW, Hilbert G, Peccoux A, Lafontaine M, Ollat N, Gomès E, Delrot S (2015) Water limitation and rootstock genotype interact to alter grape berry metabolism through transcriptome reprogramming. Horticulture research 2:15012

Bianco RL, Farina V, Avellone G, Filizzola F, Agozzino P (2008) Fruit quality and volatile fraction of 'Pink Lady'apple trees in response to rootstock vigor and partial rootzone drying. Journal of the Science of Food and Agriculture 88 (8):1325-1334

Bindon KA, Dry PR, Loveys BR (2007) Influence of Plant Water Status on the Production of C13-Norisoprenoid Precursors in Vitis vinifera L. Cv. Cabernet Sauvignon Grape Berries. Journal of Agricultural and Food Chemistry 55 (11):4493-4500. doi:10.1021/jf063331p

Callejon RM, Margulies B, Hirson GD, Ebeler SE (2012) Dynamic Changes in Volatile Compounds during Fermentation of Cabernet Sauvignon Grapes with and without Skins. American Journal of Enology and Viticulture 63 (3):301-312. doi:10.5344/ajev.2012.12009

Canuti V, Conversano M, Calzi ML, Heymann H, Matthews MA, Ebeler SE (2009) Headspace solid-phase microextraction-gas chromatography-mass spectrometry for profiling free volatile compounds in Cabernet Sauvignon grapes and wines. J Chromatogr A 1216 (15):3012-3022. doi:10.1016/j.chroma.2009.01.104

CHAPMAN DM, ROBY G, EBELER SE, GUINARD JX, MATTHEWS MA (2005) Sensory attributes of Cabernet Sauvignon wines made from vines with different water status. Australian Journal of Grape and Wine Research 11 (3):339-347

Cuadros-Inostroza A, Ruíz-Lara S, González E, Eckardt A, Willmitzer L, Peña-Cortés H (2016) GC-MS metabolic profiling of Cabernet Sauvignon and Merlot cultivars during grapevine berry development and network analysis reveals a stage-and cultivar-dependent connectivity of primary metabolites. Metabolomics 12 (2):39 
De Vos RC, Moco S, Lommen A, Keurentjes JJ, Bino RJ, Hall RD (2007) Untargeted large-scale plant metabolomics using liquid chromatography coupled to mass spectrometry. Nature protocols 2 (4):778

Deluc LG, Quilici DR, Decendit A, Grimplet J, Wheatley MD, Schlauch KA, Mérillon JM, Cushman JC, Cramer GR (2009) Water deficit alters differentially metabolic pathways affecting important flavor and quality traits in grape berries of Cabernet Sauvignon and Chardonnay. BMC genomics 10 (1):212

des Gachons CP, Leeuwen CV, Tominaga T, Soyer JP, Gaudillère JP, Dubourdieu D (2005) Influence of water and nitrogen deficit on fruit ripening and aroma potential of Vitis vinifera L cv Sauvignon blanc in field conditions. Journal of the Science of Food and Agriculture 85 (1):73-85

El-Ansary DO, Okamoto G (2007) Vine Water Relations and Quality of \&lsquo;Muscat of Alexandria\&rsquo; Table Grapes Subjected to Partial Root-zone Drying and Regulated Deficit Irrigation. Journal of the Japanese Society for Horticultural Science 76 (1):13-19. doi:10.2503/jjshs.76.13

Fang Y, Qian MC (2006) Quantification of selected aroma-active compounds in Pinot noir wines from different grape maturities. Journal of agricultural and food chemistry 54 (22):8567-8573

Ferreira V, Lopez R (2019) The Actual and Potential Aroma of Winemaking Grapes. Biomolecules 9 (12):818

Ferris H, Zheng L, Walker M (2012) Resistance of grape rootstocks to plant-parasitic nematodes. Journal of nematology 44 (4):377

Flamini R, De Rosso M, Panighel A, Dalla Vedova A, De Marchi F, Bavaresco L (2014) Profiling of grape monoterpene glycosides (aroma precursors) by ultra-high performance-liquid chromatography-high resolution mass spectrometry (UHPLC/QTOF). J Mass Spectrom 49 (12):1214-1222. doi:10.1002/jms.3441

Francis I, Newton J (2005) Determining wine aroma from compositional data. Australian Journal of Grape and Wine Research 11 (2):114-126

Gonzalez-Barreiro C, Rial-Otero R, Cancho-Grande B, Simal-Gandara J (2015) Wine aroma compounds in grapes: a critical review. Crit Rev Food Sci Nutr 55 (2):202218. doi:10.1080/10408398.2011.650336

Hampel D, Robinson AL, Johnson A, Ebeler SE (2014) Direct hydrolysis and analysis of glycosidically bound aroma compounds in grapes and wines: comparison of hydrolysis conditions and sample preparation methods. Australian journal of grape and wine research 20 (3):361-377

Harbertson JF, Keller M (2011) Rootstock Effects on Deficit-Irrigated Winegrapes in a Dry Climate: Grape and Wine Composition. American Journal of Enology and Viticulture 63 (1):40-48. doi:10.5344/ajev.2011.11079

Hjelmeland AK, Ebeler SE (2014) Glycosidically Bound Volatile Aroma Compounds in Grapes and Wine: A Review. American Journal of Enology and Viticulture 66 (1):1-11. doi:10.5344/ajev.2014.14104

Jones T, Cullis BR, Clingeleffer PR, Rühl E (2009) Effects of novel hybrid and traditional rootstocks on vigour and yield components of Shiraz grapevines. Australian Journal of Grape and Wine Research 15 (3):284-292 
Keller M, Mills LJ, Harbertson JF (2012) Rootstock effects on deficit-irrigated winegrapes in a dry climate: Vigor, yield formation, and fruit ripening. American Journal of Enology and Viticulture 63 (1):29-39

Koundouras S, Hatzidimitriou E, Karamolegkou M, Dimopoulou E, Kallithraka S, Tsialtas JT, Zioziou E, Nikolaou N, Kotseridis Y (2009) Irrigation and rootstock effects on the phenolic concentration and aroma potential of Vitis vinifera L. cv. cabernet sauvignon grapes. J Agric Food Chem 57 (17):7805-7813. doi:10.1021/jf901063a

Lee J-E, Hwang G-S, Van Den Berg F, Lee C-H, Hong Y-S (2009) Evidence of vintage effects on grape wines using 1H NMR-based metabolomic study. Analytica chimica acta 648 (1):71-76

Liu D, Xing R-R, Li Z, Yang D-M, Pan Q-H (2016) Evolution of volatile compounds, aroma attributes, and sensory perception in bottle-aged red wines and their correlation. European Food Research and Technology 242 (11):1937-1948. doi:10.1007/s00217-016-2693-1

Main G, Morris J, Striegler K (2002) Rootstock effects on Chardonel productivity, fruit, and wine composition. American Journal of Enology and Viticulture 53 (1):37-40

Narduzzi L, Stanstrup J, Mattivi F (2015) Comparing Wild American Grapes with Vitis vinifera: A Metabolomics Study of Grape Composition. J Agric Food Chem 63 (30):6823-6834. doi:10.1021/acs.jafc.5b01999

Nelson CC, Kennedy JA, Zhang Y, Kurtural SK (2015) Applied Water and Rootstock Affect Productivity and Anthocyanin Composition of Zinfandel in Central California. American Journal of Enology and Viticulture 67 (1):18-28. doi:10.5344/ajev.2015.15043

Noble A, Cole V (2003) Flavor chemistry. In: Fermented Beverage Production. Springer, pp 393-412

Olarte Mantilla SM, Collins C, Iland PG, Kidman CM, Ristic R, Boss PK, Jordans C, Bastian SEP (2018) Shiraz (<em>Vitis vinifera</em> L.) Berry and Wine Sensory Profiles and Composition Are Modulated by Rootstocks. American Journal of Enology and Viticulture 69 (1):32-44. doi:10.5344/ajev.2017.17017

Ollat N, Bordenave L, Tandonnet J-P, Boursiquot J-M, Marguerit E Grapevine rootstocks: origins and perspectives. In: I International Symposium on Grapevine Roots 1136, 2014. pp 11-22

Ough C, Cook J, Lider L (1968) Rootstock-scion interactions concerning wine making. II. Wine compositional and sensory changes attributed to rootstock and fertilizer level differences. American Journal of Enology and Viticulture 19 (4):254-265

Parker M, Capone DL, Francis IL, Herderich MJ (2017) Aroma Precursors in Grapes and Wine: Flavor Release during Wine Production and Consumption. J Agric Food Chem. doi:10.1021/acs.jafc.6b05255

Plata C, Millan C, Mauricio J, Ortega J (2003) Formation of ethyl acetate and isoamyl acetate by various species of wine yeasts. Food Microbiology 20 (2):217-224

Qian MC, Fang Y, Shellie K (2009) Volatile Composition of Merlot Wine from Different Vine Water Status. Journal of Agricultural and Food Chemistry 57 (16):74597463. doi:10.1021/jf9009558 
Reisch BI, Owens CL, Cousins PS (2012) Grape. In: Fruit breeding. Springer, pp 225262

Reynolds AG, Lowrey WD, Tomek L, Hakimi J, De Savigny C (2007) Influence of irrigation on vine performance, fruit composition, and wine quality of Chardonnay in a cool, humid climate. American Journal of Enology and Viticulture 58 (2):217-228

Romero P, Botía P, del Amor FM, Gil-Muñoz R, Flores P, Navarro JM (2019) Interactive effects of the rootstock and the deficit irrigation technique on wine composition, nutraceutical potential, aromatic profile, and sensory attributes under semiarid and water limiting conditions. Agricultural Water Management 225:105733. doi:https://doi.org/10.1016/j.agwat.2019.105733

Roullier-Gall C, Witting M, Gougeon RD, Schmitt-Kopplin P (2014) High precision mass measurements for wine metabolomics. Frontiers in Chemistry 2 (102). doi:10.3389/fchem.2014.00102

Schrimpe-Rutledge AC, Codreanu SG, Sherrod SD, McLean JA (2016) Untargeted Metabolomics Strategies - Challenges and Emerging Directions. Journal of The American Society for Mass Spectrometry 27 (12):1897-1905. doi:10.1007/s13361-016-1469-y

Schueuermann C, Khakimov B, Engelsen SB, Bremer P, Silcock P (2016) GC-MS Metabolite Profiling of Extreme Southern Pinot noir Wines: Effects of Vintage, Barrel Maturation, and Fermentation Dominate over Vineyard Site and Clone Selection. Journal of Agricultural and Food Chemistry 64 (11):2342-2351. doi:10.1021/acs.jafc.5b05861

Schueuermann C, Steel CC, Blackman JW, Clark AC, Schwarz LJ, Moraga J, Collado IG, Schmidtke LM (2019) A GC-MS untargeted metabolomics approach for the classification of chemical differences in grape juices based on fungal pathogen. Food chemistry 270:375-384

Serra I, Strever A, Myburgh P, Deloire A (2014) the interaction between rootstocks and cultivars (V itis vinifera L.) to enhance drought tolerance in grapevine. Australian Journal of Grape and Wine Research 20 (1):1-14

Shure KB, Acree TE (1994) Changes in the odor-active compounds in Vitis labruscana cv. Concord during growth and development. Journal of agricultural and food chemistry 42 (2):350-353

Slaghenaufi D, Guardini S, Tedeschi R, Ugliano M (2019) Volatile terpenoids, norisoprenoids and benzenoids as markers of fine scale vineyard segmentation for Corvina grapes and wines. Food Research International 125:108507. doi:https://doi.org/10.1016/j.foodres.2019.108507

Stockert CM, Bisson LF, Adams DO, Smart DR (2013) Nitrogen status and fermentation dynamics for Merlot on two rootstocks. American Journal of Enology and Viticulture 64 (2):195-202

Šuklje K, Carlin S, Stanstrup J, Antalick G, Blackman JW, Meeks C, Deloire A, Schmidtke LM, Vrhovsek U (2019) Unravelling wine volatile evolution during Shiraz grape ripening by untargeted HS-SPME-GC $\times$ GC-TOFMS. Food chemistry 277:753-765 
Sun Q, Sacks G, Lerch S, Heuvel JEV (2011) Impact of shoot thinning and harvest date on yield components, fruit composition, and wine quality of Marechal Foch. American journal of enology and viticulture 62 (1):32-41

Tautenhahn R, Patti GJ, Rinehart D, Siuzdak G (2012) XCMS Online: a web-based platform to process untargeted metabolomic data. Analytical chemistry 84 (11):5035-5039

Teh SL, Rostandy B, Awale M, Luby JJ, Fennell A, Hegeman AD (2019) Genetic analysis of stilbenoid profiles in grapevine stems reveals a major mQTL hotspot on chromosome 18 associated with disease-resistance motifs. Horticulture research $6(1): 1-11$

Thomas AL, Harris JL, Bergmeier EA, Striegler RK (2017) Establishment Technique and Rootstock Impact 'Chambourcin'Grapevine Morphology and Production in Missouri. HortTechnology 27 (2):248-256

Toffali K, Zamboni A, Anesi A, Stocchero M, Pezzotti M, Levi M, Guzzo F (2011) Novel aspects of grape berry ripening and post-harvest withering revealed by untargeted LC-ESI-MS metabolomics analysis. Metabolomics 7 (3):424-436. doi:10.1007/s11306-010-0259-y

Tramontini S, Vitali M, Centioni L, Schubert A, Lovisolo C (2013) Rootstock control of scion response to water stress in grapevine. Environmental and experimental botany 93:20-26

Van Wyk C, Augustyn O, De Wet P, Joubert W (1979) Isoamyl acetate - a key fermentation volatile of wines of Vitis vinifera cv Pinotage. American Journal of Enology and Viticulture 30 (3):167-173

Vivian Z, Thibaut V, Agnès D, Sandrine B, Fabrice L, Carole K, Katia G, Jorge ES, Olivier V, Jean-Laurent S (2018) The impact of plant water status on the gas exchange, berry composition and wine quality of Chasselas grapes in Switzerland. OENO One 52 (4). doi:10.20870/oeno-one.2018.52.4.2181

Wang Y, Chen W-K, Gao X-T, He L, Yang X-H, He F, Duan C-Q, Wang J (2019) Rootstock-Mediated Effects on Cabernet Sauvignon Performance: Vine Growth, Berry Ripening, Flavonoids, and Aromatic Profiles. International Journal of Molecular Sciences 20 (2):401

Warschefsky EJ, Klein LL, Frank MH, Chitwood DH, Londo JP, von Wettberg EJ, Miller AJ (2016) Rootstocks: diversity, domestication, and impacts on shoot phenotypes. Trends in plant science 21 (5):418-437

Wooldridge J, Louw P, Conradie W (2010) Effects of rootstock on grapevine performance, petiole and must composition, and overall wine score of Vitis vinifera cv. Chardonnay and Pinot Noir. South African Journal of Enology and Viticulture 31 (1):45-48

Young PR, Eyeghe-Bickong HA, du Plessis K, Alexandersson E, Jacobson DA, Coetzee Z, Deloire A, Vivier MA (2016) Grapevine plasticity in response to an altered microclimate: Sauvignon Blanc modulates specific metabolites in response to increased berry exposure. Plant physiology 170 (3):1235-1254

Zhang L, Marguerit E, Rossdeutsch L, Ollat N, Gambetta GA (2016) The influence of grapevine rootstocks on scion growth and drought resistance. Theoretical and Experimental Plant Physiology 28 (2):143-157. doi:10.1007/s40626-016-0070-x 
Figures:

\section{Subtle Differentiation Metabolomics Workflow}

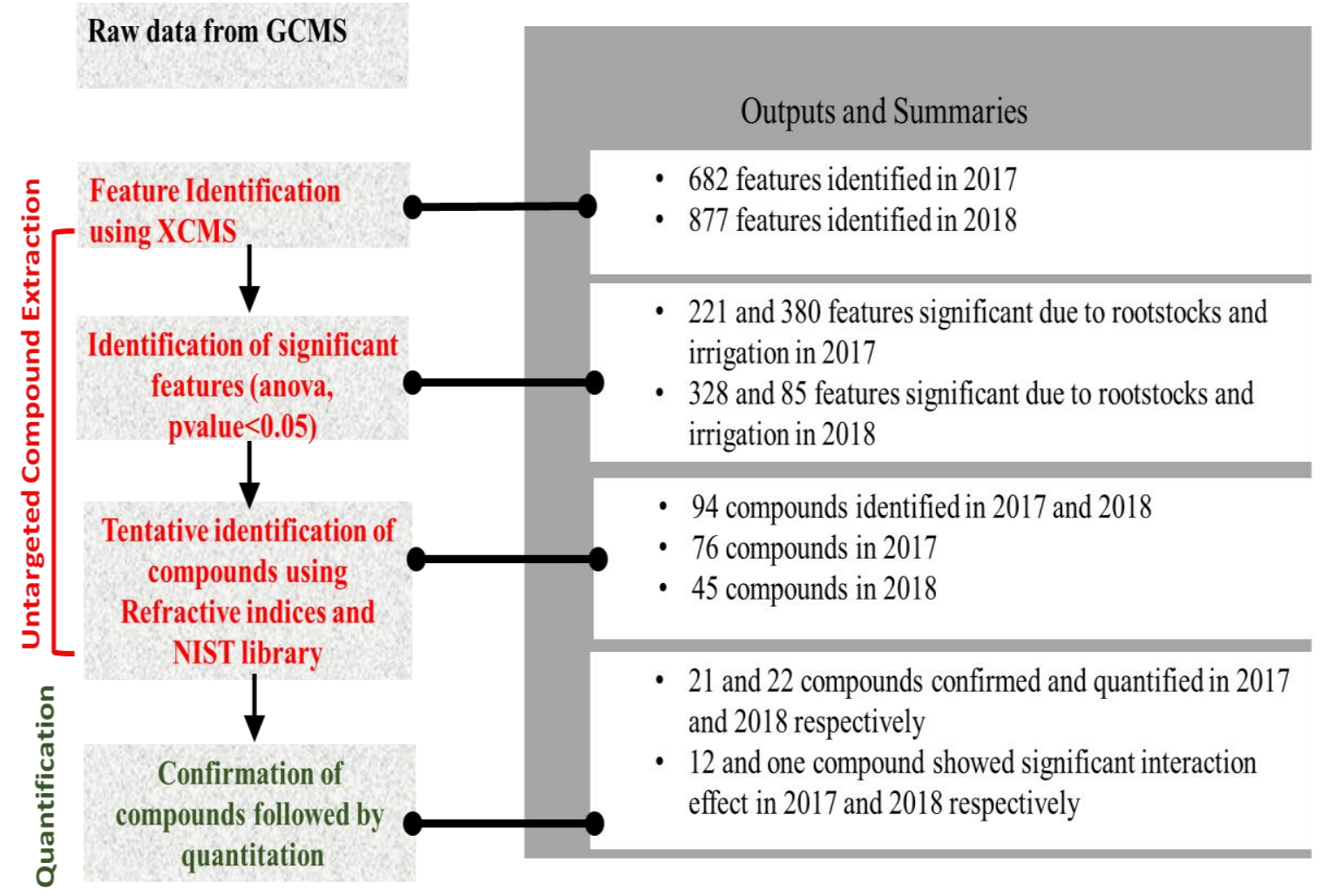

Figure 3.1: Flowchart of the Subtle Differentiation Metabolomics workflow to identify the minute differences in wine aroma due to rootstocks and irrigation. 


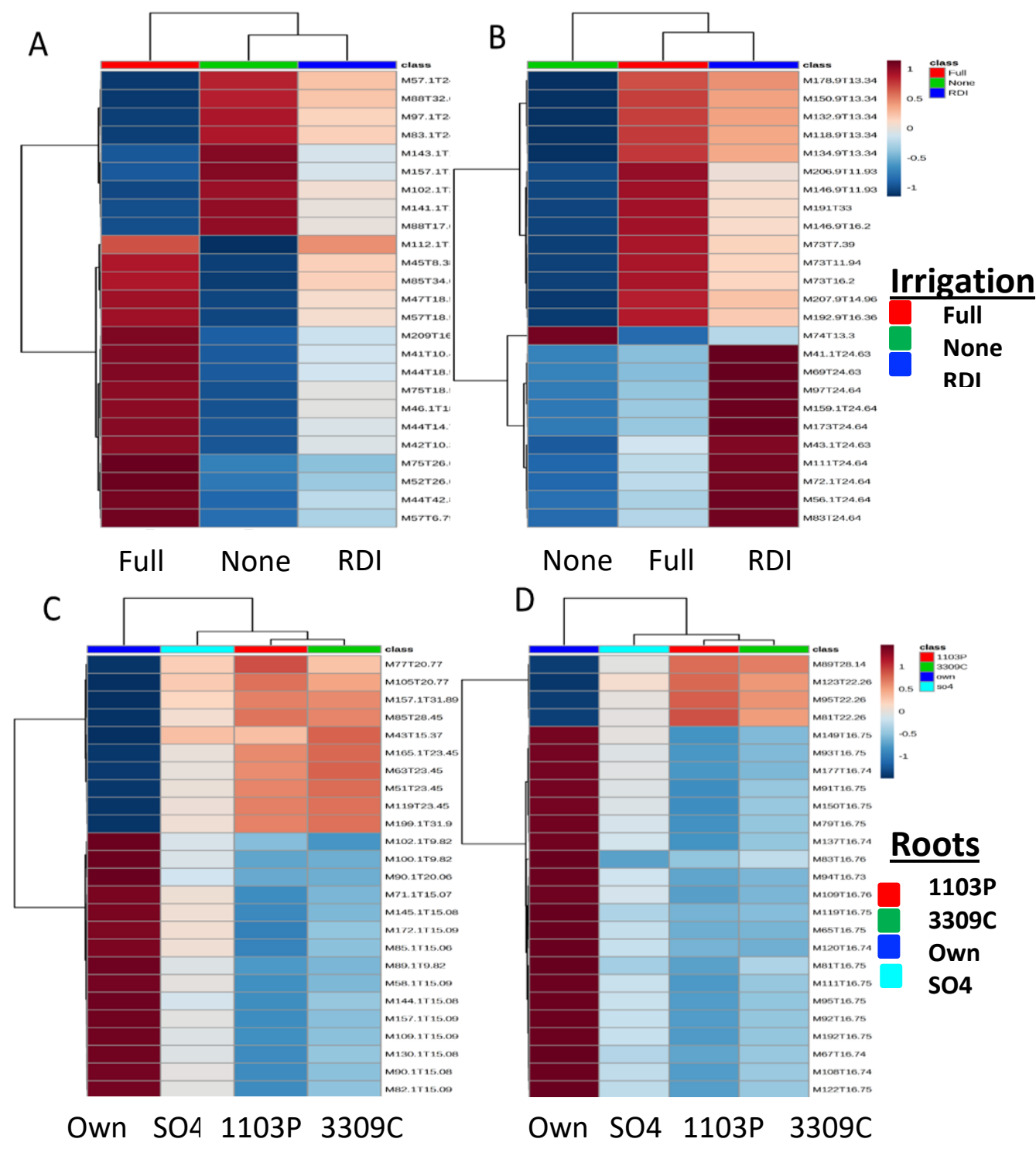

Figure 3.2: Heatmaps of the top 25 most influential features for differentiating wine volatiles by (A) irrigation regime in 2017, (B) irrigation regime in 2018, (C) rootstocks in 2017 and (D) rootstocks in 2018. While only top contributors are shown the heatmaps were generated using all the features. The rows in the heatmap represent features $(\mathrm{M}(\mathrm{m} / \mathrm{z}) \mathrm{T}$ (time in minutes) and the columns indicate sample categories. The colors of the heatmap cells indicate the abundance of compounds across different samples. The color gradient, ranging from dark blue through white to dark red, represents low, middle and high abundance of a compound. 
A

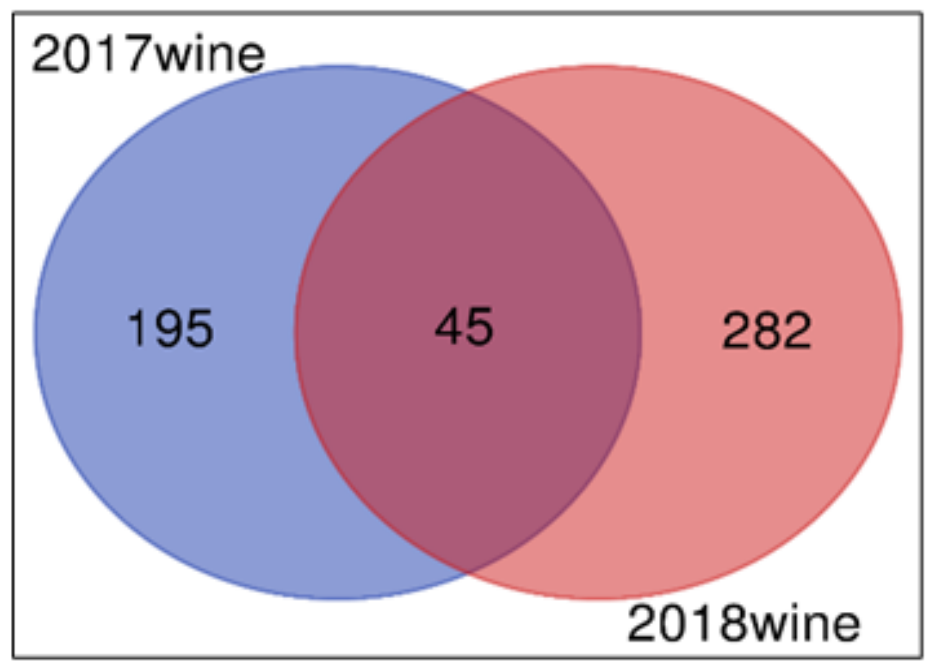

B

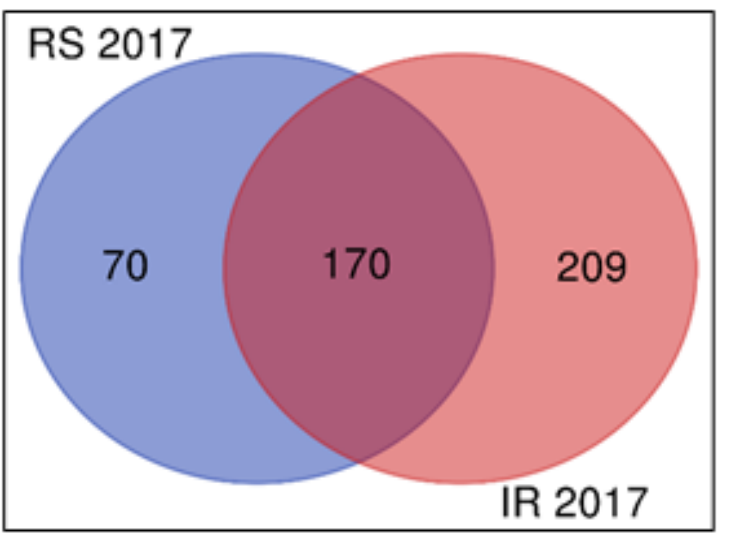

C

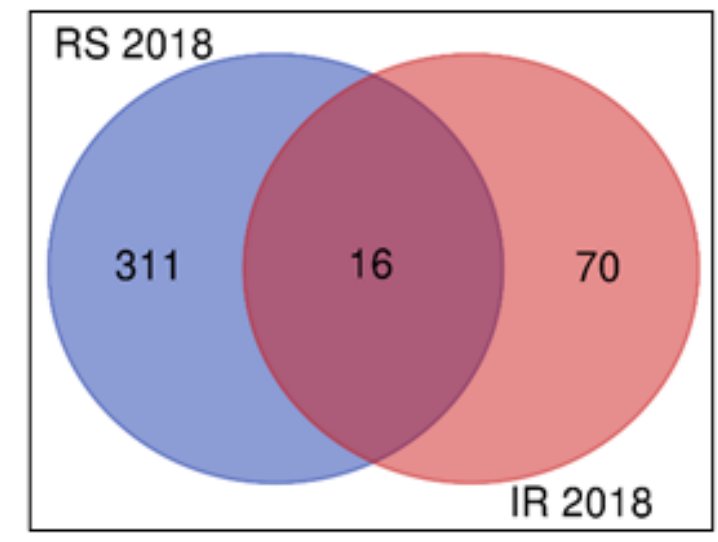

Figure 3.3: A) Venn diagram showing common and unique features between wines in 2017 and 2018. Only 45 features were common in both years showing yearly differences in metabolites. B) Venn diagram showing common and unique features between rootstocks and irrigation treatments in wines in 2017. 170 features were similar between rootstocks and irrigation treatments. C) Venn diagram showing common and unique features between rootstock and irrigation treatments in wines in 2018. Few features were similar between rootstocks and irrigation treatments. 

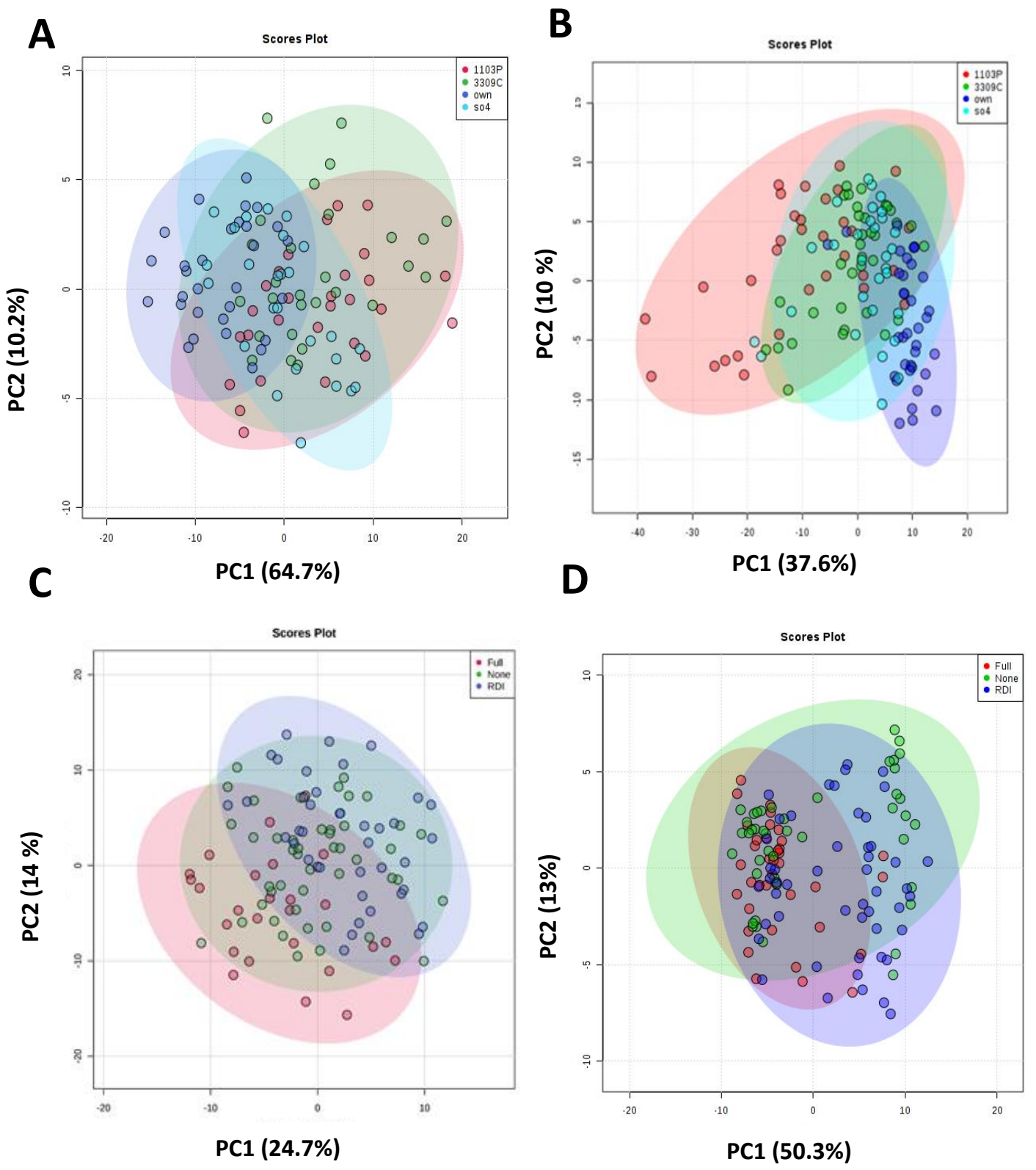

Figure 3.4: PCA Scores plot of wine metabolite features separated by rootstocks in (A) 2017 and (B) in 2018 regardless of the irrigation treatment. PCA Scores plot of wine metabolite features separated by irrigation treatments in (C) 2017 and (D) 2018 regardless of the rootstock treatment. PCA was performed using logtransformed and autoscaled significant features. 


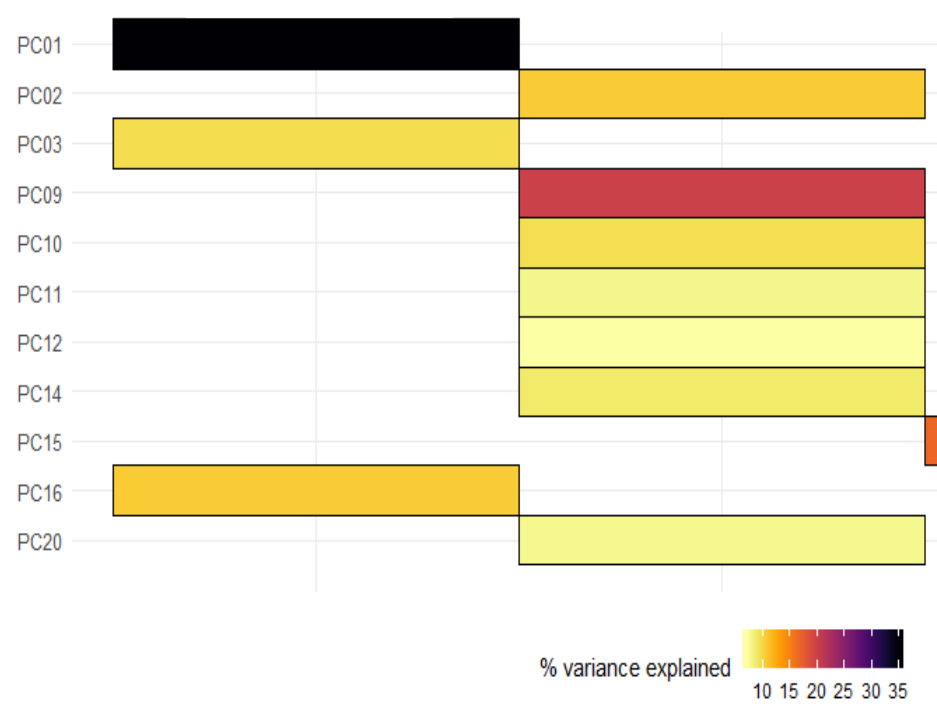

\section{8}

\section{Rootstock}

Irrigation

Rootstock x Irrigation
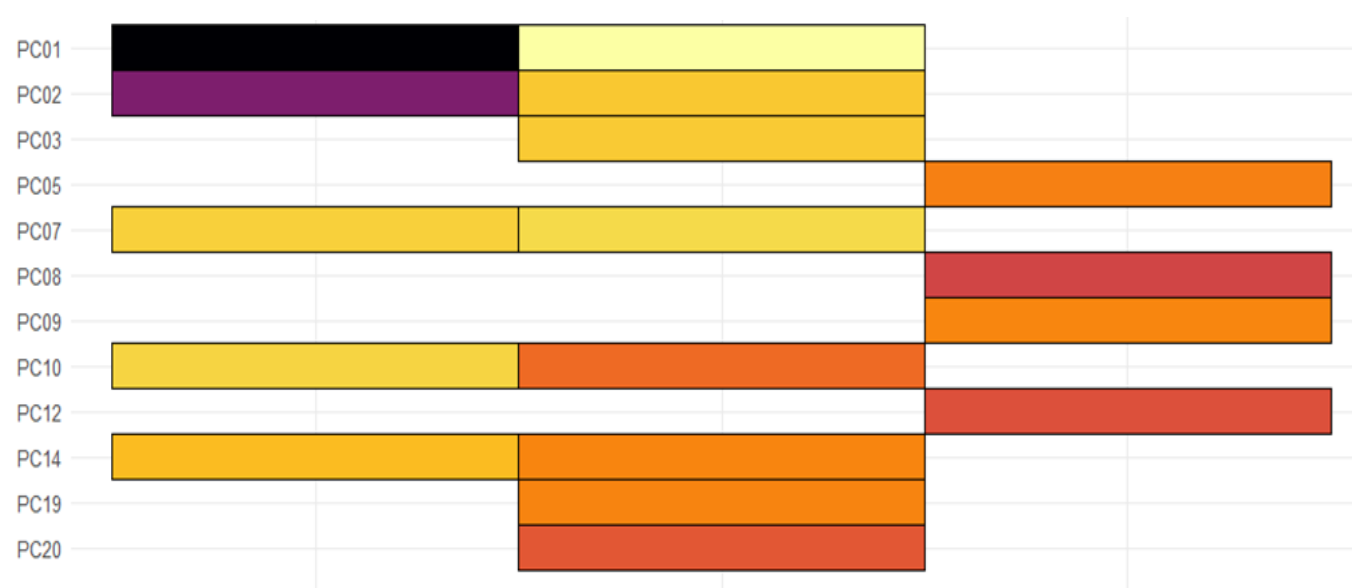

$\%$ variance explained

$10 \quad 20 \quad 30$

Figure 3.5: A linear model was estimated for wine 2017 and 2018 PCs 1 to 20. The model included rootstock (own-rooted, '1103P', '3309C', 'SO4'), irrigation (Full, RDI, None), and rootstock by irrigation interaction. The 20 PCs capture $85.79 \%$ and $78.34 \%$ of the variance in 2017 and 2018 respectively. Only factors which explained a significant portion of the variance $(\mathrm{p}<0.05)$ are plotted. The percent variation explained by each factor is indicated using color gradient. 
A

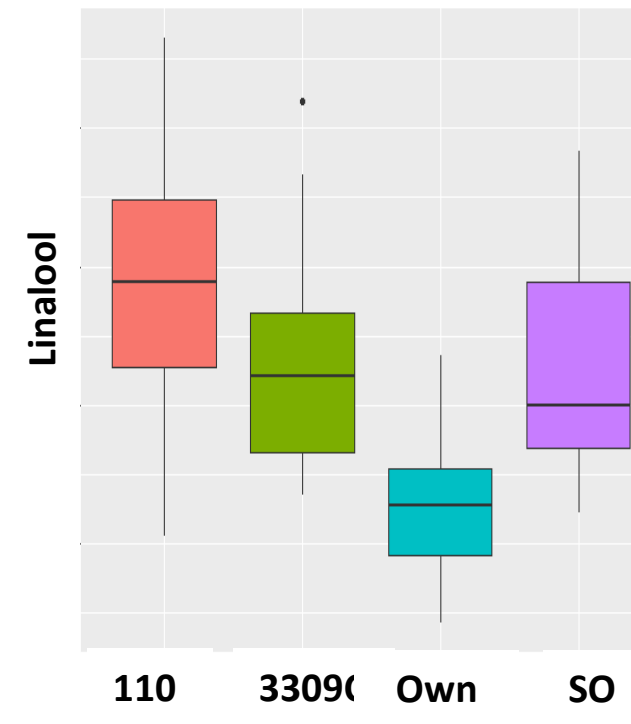

B

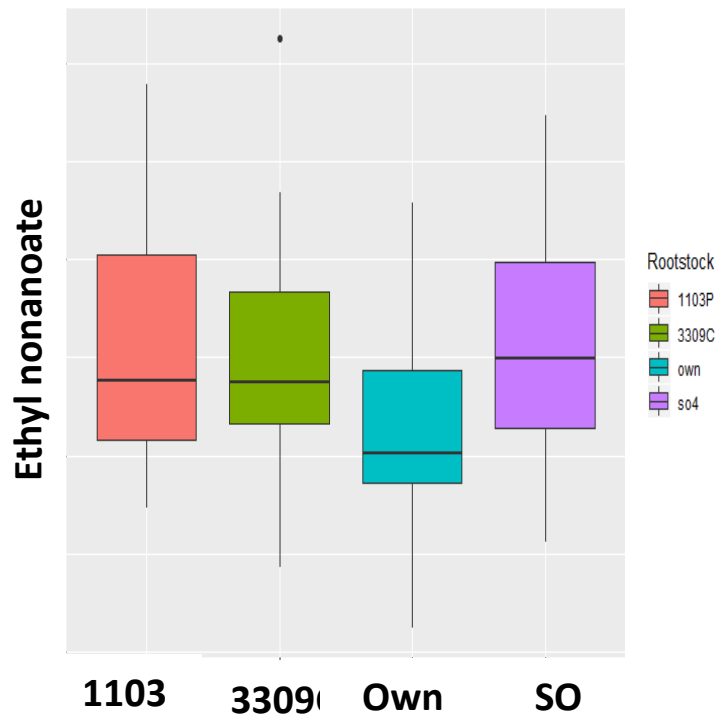

Figure 3.6: Boxplots of (A) $\beta$-Linalool (Linalool) and (B) Ethyl hexanoate concentrations differences in rootstocks and own-rooted Chambourcin. Rootstocks increased concentrations of Linalool and Ethyl nonanoate in wines 2018. Concentrations were measured in $\mathrm{ppb}(\mathrm{ug} / \mathrm{L})$. 


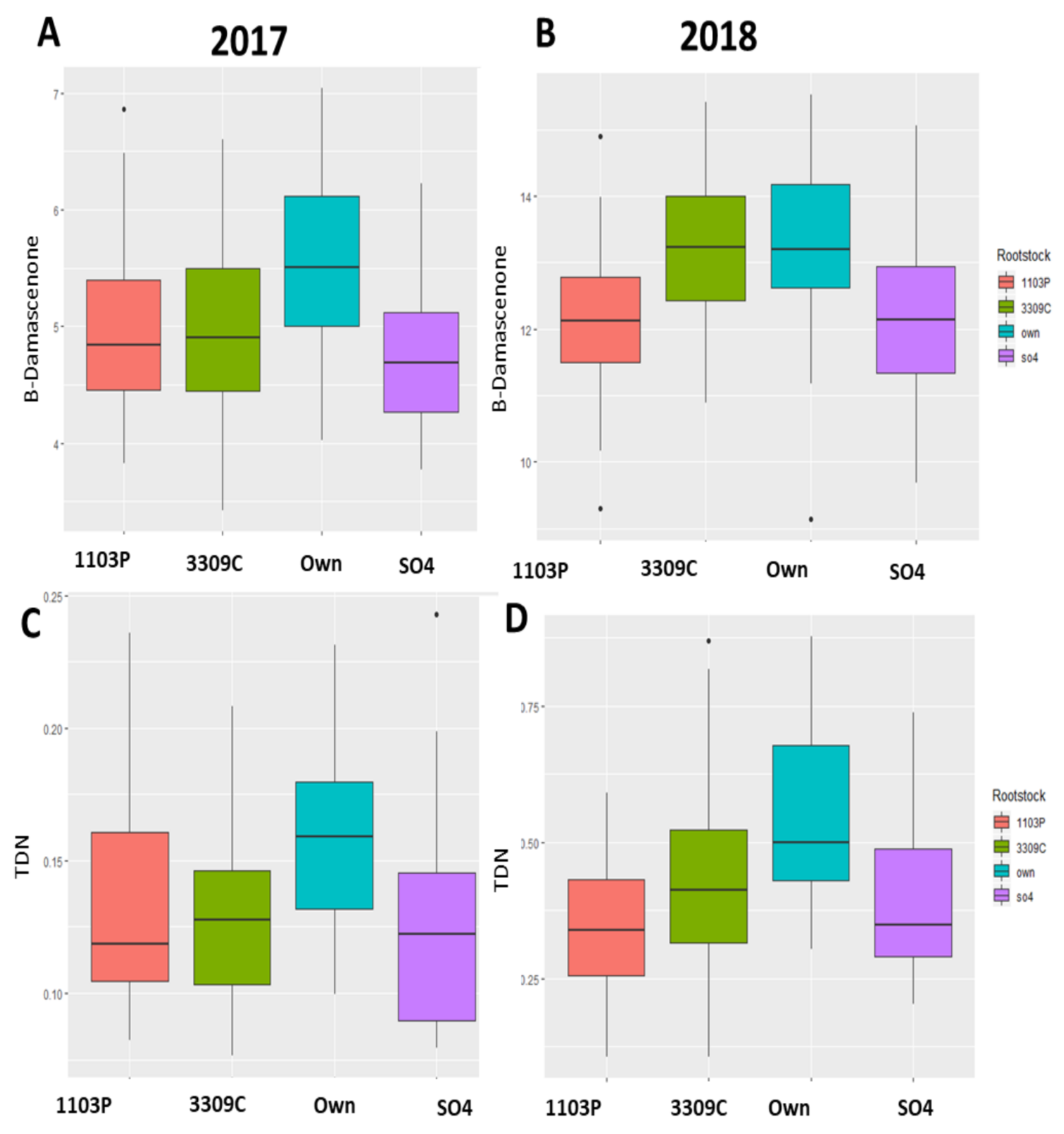

Figure 3.7: A) Boxplots of $\beta$-Damascenone concentrations differences in rootstocks and own-rooted Chambourcin in 2017 and B) in 2018. C)Boxplots of 1,1,6-trimethyl1,2-dihydronapthalene (TDN) concentrations differences in rootstocks and own-rooted Chambourcin in 2017 B) in 2018. Both $\beta$-Damascenone TDN concentrations were found in higher in own-rooted Chambourcin in wines in both 2017 and 2018. Concentrations were measured in $\mathrm{ppb}(\mathrm{ug} / \mathrm{L})$. 


\section{Tables:}

Table 3.1: Mean concentrations of wine volatiles in 2017 and 2018 as an Interaction effect between rootstocks and irrigation observed in wine volatiles in 2017 and 2018. The concentration was measured in ug/L. The concentration was measured in ug/L. Different letters within a row indicate significant differences for Duncan's Multiple Range test at $\mathrm{p}<0.05$ among the rootstock irrigation interaction effect.

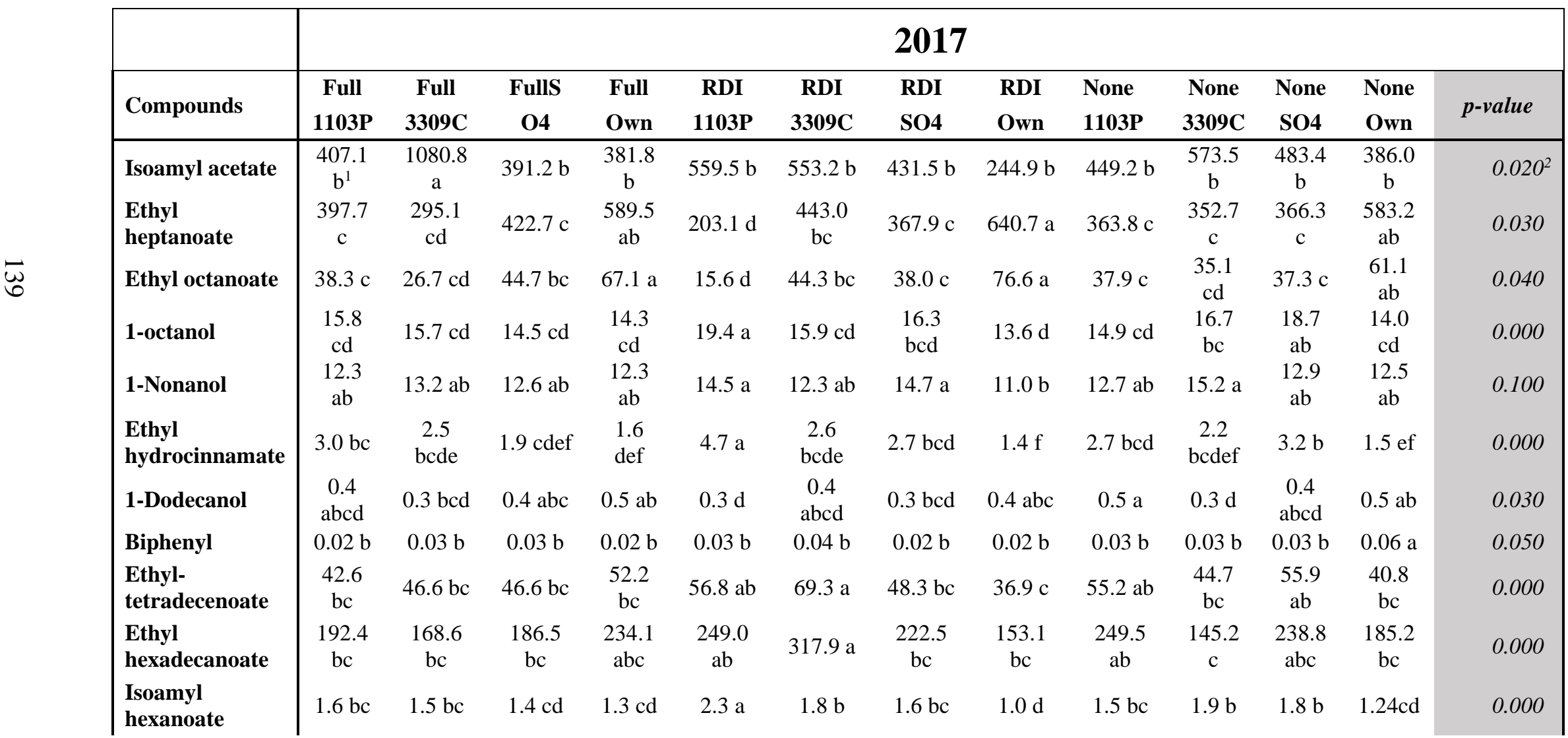




\begin{tabular}{|c|c|c|c|c|c|c|c|c|c|c|c|c|c|}
\hline $\begin{array}{l}\text { 2-phenylethyl } \\
\text { acetate }\end{array}$ & $1.6 \mathrm{bc}$ & $\begin{array}{l}35.2 \\
\text { bcd }\end{array}$ & $\begin{array}{l}28.6 \\
\text { cde }\end{array}$ & $\begin{array}{l}28.2 \\
\text { cde }\end{array}$ & $60.4 \mathrm{a}$ & $41.8 \mathrm{bc}$ & $\begin{array}{l}39.8 \\
\text { bcd }\end{array}$ & $15.7 \mathrm{e}$ & $\begin{array}{l}35.2 \\
\text { bcd }\end{array}$ & $\begin{array}{c}46.70 \\
\mathrm{~b}\end{array}$ & $\begin{array}{l}39.1 \\
\text { bcd }\end{array}$ & $\begin{array}{c}24.9 \\
\mathrm{de}\end{array}$ & 0.000 \\
\hline & \multicolumn{13}{|c|}{2018} \\
\hline Linalool & $15.6 \mathrm{a}$ & $\begin{array}{l}11.5 \\
\text { bcd }\end{array}$ & $9.7 \mathrm{~cd}$ & $8.4 \mathrm{de}$ & $16.1 \mathrm{a}$ & $\begin{array}{l}10.6 \\
\text { bcd }\end{array}$ & $\begin{array}{l}11.4 \\
\text { bcd }\end{array}$ & $5.6 \mathrm{e}$ & $\begin{array}{l}11.5 \\
\text { bcd }\end{array}$ & $\begin{array}{l}12.1 \\
\mathrm{bc}\end{array}$ & $\begin{array}{l}13.4 \\
\mathrm{ab}\end{array}$ & $6.1 \mathrm{e}$ & 0.005 \\
\hline
\end{tabular}

${ }^{1}$ Values represent $\mu \mathrm{g} / \mathrm{L}$ concentration generated by comparing the peak area, relative to the internal standard (2Octanol), and comparing to the calibration curve generated with an authentic standard.

${ }^{2}$ Analysis of variance was used to compare data with Rootstocks, Irrigation and Rootstock x Irrigation as factors 
Table 3.2: Concentrations of Wine volatiles quantified $(\mu \mathrm{g} / \mathrm{L}(\mathrm{ppb}))$ in 2017 and 2018 using GC-MS/MS. ANOVA for significant wine volatiles for the main effect of rootstocks and irrigations (highlighted in pink) regardless of rootstock and irrigation interaction effect. ANOVA was used to compare data with rootstocks, irrigation, and rootstock x irrigation as factors; different letters within a row indicate significant differences for Duncan's Multiple Range test at $\mathrm{p}<0.05$.

\begin{tabular}{|c|c|c|c|c|c|c|c|c|c|c|c|c|c|c|c|c|c|c|c|}
\hline $\begin{array}{c}\text { Compou } \\
\text { nds }\end{array}$ & $\begin{array}{c}\text { odor } \\
\text { thres } \\
\text { hold } \\
(\mathrm{ug} / \mathrm{L})\end{array}$ & $\begin{array}{c}1103 \\
P\end{array}$ & $\begin{array}{c}3309 \\
\mathrm{C}\end{array}$ & SO4 & Own & $\begin{array}{c}\text { p- } \\
\text { value }\end{array}$ & Full & RDI & None & $\begin{array}{c}\text { p- } \\
\text { valu } \\
\text { e }\end{array}$ & $\begin{array}{c}1103 \\
P\end{array}$ & $\begin{array}{c}3309 \\
\mathrm{C}\end{array}$ & SO4 & Own & $\begin{array}{c}\text { p- } \\
\text { value }\end{array}$ & Full & RDI & None & $\begin{array}{c}\text { p- } \\
\text { value }\end{array}$ \\
\hline $\begin{array}{c}\text { Damasce } \\
\text { none }\end{array}$ & 0.05 & $\begin{array}{l}5.007 \\
\mathrm{a}\end{array}$ & $\begin{array}{l}4.56 \\
\mathrm{a}\end{array}$ & $\begin{array}{c}4.74 \\
\mathrm{a}\end{array}$ & $\begin{array}{c}5.72 \\
b\end{array}$ & $\begin{array}{r}8.2 \mathrm{E}- \\
06\end{array}$ & $\begin{array}{l}4.82 \\
\mathrm{a}\end{array}$ & $\begin{array}{l}5.33 \\
b\end{array}$ & $\begin{array}{l}4.96 \\
a b\end{array}$ & $\begin{array}{r}0.04 \\
8\end{array}$ & $\begin{array}{c}12.05 \\
\mathrm{~b}\end{array}$ & $\begin{array}{c}13.05 \\
\mathrm{a}\end{array}$ & $\begin{array}{c}12.15 \\
b\end{array}$ & $\begin{array}{c}13.26 \\
\mathrm{a}\end{array}$ & 0.008 & $12.42 \mathrm{a}$ & $\begin{array}{c}12.73 \\
\mathrm{a}\end{array}$ & $12.74 \mathrm{a}$ & 0.577 \\
\hline $\begin{array}{l}\text { Isoamyl } \\
\text { acetate }\end{array}$ & $17-30$ & $\begin{array}{l}478.4 \\
3 \mathrm{a}\end{array}$ & $\begin{array}{l}764.6 \\
4 \mathrm{~b}\end{array}$ & $\begin{array}{l}436.9 \\
1 \mathrm{~b}\end{array}$ & $\begin{array}{l}340.2 \\
1 \mathrm{~b}\end{array}$ & $\begin{array}{r}2.75 \mathrm{E} \\
-05\end{array}$ & $\begin{array}{l}588.5 \\
7 \mathrm{a}\end{array}$ & $\begin{array}{l}439.7 \\
3 \mathrm{a}\end{array}$ & $\begin{array}{l}471.0 \\
9 a\end{array}$ & $\begin{array}{r}0.55 \\
23\end{array}$ & $\begin{array}{c}331.7 \\
3 \mathrm{a}\end{array}$ & $\begin{array}{c}297.0 \\
5 \mathrm{a}\end{array}$ & $\begin{array}{c}287.8 \\
9 \mathrm{a}\end{array}$ & $\begin{array}{c}277.3 \\
5 \mathrm{a}\end{array}$ & 0.454 & $\begin{array}{c}311.92 \\
\mathrm{a}\end{array}$ & $\begin{array}{c}269.8 \\
\mathrm{a}\end{array}$ & $\begin{array}{c}315.98 \\
\mathrm{a}\end{array}$ & 0.164 \\
\hline $\begin{array}{l}\text { Terpinol } \\
\text { ene } \\
2-\end{array}$ & $\begin{array}{l}14 \\
\mathrm{mg} / \mathrm{L}\end{array}$ & $\begin{array}{l}0.474 \\
\mathrm{a}\end{array}$ & $\begin{array}{l}0.426 \\
a\end{array}$ & $\begin{array}{l}0.382 \\
\mathrm{a}\end{array}$ & $\begin{array}{l}0.433 \\
\mathrm{a}\end{array}$ & 0.289 & $\begin{array}{l}0.419 \\
\text { ab }\end{array}$ & $\begin{array}{l}0.487 \\
\mathrm{a}\end{array}$ & $\begin{array}{l}0.384 \\
\text { b }\end{array}$ & $\begin{array}{r}0.04 \\
76\end{array}$ & $\begin{array}{c}1.11 \\
\mathrm{a}\end{array}$ & $1.08 \mathrm{a}$ & $1.35 \mathrm{a}$ & $1.16 \mathrm{a}$ & $\begin{array}{c}0.317 \\
7\end{array}$ & $1.31 \mathrm{a}$ & $0.99 \mathrm{~b}$ & $1.27 \mathrm{a}$ & $\begin{array}{c}0.029 \\
1\end{array}$ \\
\hline $\begin{array}{c}\text { Octanon } \\
\text { e } \\
\text { Methyl }\end{array}$ & 190 & $\begin{array}{l}0.234 \\
\mathrm{a}\end{array}$ & $\begin{array}{l}0.269 \\
\mathrm{a}\end{array}$ & $\begin{array}{l}0.224 \\
\mathrm{a}\end{array}$ & $\begin{array}{l}0.237 \\
\mathrm{a}\end{array}$ & 0.711 & $\begin{array}{l}0.224 \\
\mathrm{a}\end{array}$ & $\begin{array}{l}0.270 \\
\mathrm{a}\end{array}$ & $\begin{array}{l}0.231 \\
\mathrm{a}\end{array}$ & $\begin{array}{r}0.33 \\
9\end{array}$ & $\begin{array}{c}11.73 \\
\mathrm{a}\end{array}$ & $\begin{array}{c}11.79 \\
\mathrm{a}\end{array}$ & $\begin{array}{c}11.38 \\
\mathrm{a}\end{array}$ & $\begin{array}{c}11.54 \\
\mathrm{a}\end{array}$ & 0.976 & $11.61 \mathrm{a}$ & $\begin{array}{c}11.61 \\
\mathrm{a}\end{array}$ & $11.62 \mathrm{a}$ & 0.999 \\
\hline $\begin{array}{c}\text { octanoat } \\
\text { e } \\
\text { Ethyl }\end{array}$ & 200 & $\begin{array}{l}0.343 \\
\mathrm{a}\end{array}$ & $\begin{array}{l}0.349 \\
\mathrm{a}\end{array}$ & $\begin{array}{l}0.425 \\
\mathrm{a}\end{array}$ & $\begin{array}{l}0.694 \\
\text { b }\end{array}$ & $\begin{array}{r}8.70 \mathrm{E} \\
-11\end{array}$ & $\begin{array}{l}0.436 \\
\mathrm{a}\end{array}$ & $\begin{array}{l}0.466 \\
\mathrm{a}\end{array}$ & $\begin{array}{l}0.473 \\
\mathrm{a}\end{array}$ & $\begin{array}{r}0.66 \\
6\end{array}$ & $\begin{array}{c}1.46 \\
\mathrm{c}\end{array}$ & $2.01 \mathrm{~b}$ & $\begin{array}{l}2.07 \\
\mathrm{ab}\end{array}$ & $2.51 \mathrm{a}$ & $\begin{array}{c}0.000 \\
262\end{array}$ & $1.91 \mathrm{~b}$ & $1.81 \mathrm{~b}$ & $2.30 \mathrm{a}$ & $\begin{array}{c}0.029 \\
11\end{array}$ \\
\hline $\begin{array}{l}\text { heptanoa } \\
\text { te } \\
\text { Ethyl }\end{array}$ & 2 & $\begin{array}{l}316.6 \\
5 \mathrm{a}\end{array}$ & $\begin{array}{l}356.5 \\
1 \mathrm{a}\end{array}$ & $\begin{array}{l}385.0 \\
3 \mathrm{a}\end{array}$ & $\begin{array}{l}603.4 \\
2 \mathrm{~b}\end{array}$ & $\begin{array}{r}6.18 \mathrm{E} \\
-11\end{array}$ & $\begin{array}{l}428.4 \\
2 \mathrm{a}\end{array}$ & $\begin{array}{l}413.3 \\
8 \mathrm{a}\end{array}$ & $\begin{array}{l}420.2 \\
1 \mathrm{a}\end{array}$ & $\begin{array}{r}0.95 \\
8\end{array}$ & $\begin{array}{c}944.6 \\
\mathrm{a}\end{array}$ & $\begin{array}{l}1154 . \\
75 \mathrm{~b}\end{array}$ & $\begin{array}{l}1186 . \\
22 \mathrm{~b}\end{array}$ & $\begin{array}{l}1306 . \\
46 \mathrm{~b}\end{array}$ & $\begin{array}{c}0.005 \\
5\end{array}$ & $\begin{array}{c}1156.5 \\
2 \mathrm{ab}\end{array}$ & $\begin{array}{l}1056 . \\
98 \mathrm{~b}\end{array}$ & $\begin{array}{c}1229.2 \\
8 \mathrm{a}\end{array}$ & 0.17 \\
\hline $\begin{array}{l}\text { octanoat } \\
\mathrm{e}\end{array}$ & 5 & $\begin{array}{l}30.38 \\
1 \mathrm{a}\end{array}$ & $\begin{array}{l}34.54 \\
7 \mathrm{a}\end{array}$ & $\begin{array}{l}39.92 \\
\mathrm{a}\end{array}$ & $\begin{array}{l}68.04 \\
\text { b }\end{array}$ & $\begin{array}{r}2.11 \mathrm{E} \\
-10\end{array}$ & $\begin{array}{l}44.46 \\
\mathrm{a}\end{array}$ & $\begin{array}{l}43.75 \\
\mathrm{a}\end{array}$ & $\begin{array}{l}43.26 \\
a\end{array}$ & $\begin{array}{r}0.97 \\
7\end{array}$ & $\begin{array}{c}256.7 \\
9 \mathrm{a}\end{array}$ & $\begin{array}{l}331.6 \\
9 \mathrm{~b}\end{array}$ & $342 \mathrm{~b}$ & $\begin{array}{c}390.6 \\
\mathrm{~b}\end{array}$ & $\begin{array}{c}0.001 \\
62\end{array}$ & $\begin{array}{c}329.09 \\
\mathrm{a}\end{array}$ & $\begin{array}{c}307.6 \\
9 \mathrm{a}\end{array}$ & $\begin{array}{c}353.48 \\
\mathrm{a}\end{array}$ & $\begin{array}{c}0.276 \\
5\end{array}$ \\
\hline $\begin{array}{c}\text { Ethyl } \\
\text { nonanoat } \\
\mathrm{e}\end{array}$ & 377 & $\begin{array}{l}2.6 \mathrm{a} \\
10.04\end{array}$ & $\begin{array}{l}1.69 \\
\mathrm{~b} \\
9.033\end{array}$ & $\begin{array}{l}1.98 \\
b \\
8.61\end{array}$ & $\begin{array}{l}1.11 \\
\mathrm{c} \\
8.37\end{array}$ & $\begin{array}{r}1.57 \mathrm{E} \\
-05 \\
6.78 \mathrm{E}\end{array}$ & $\begin{array}{l}1.66 \\
\mathrm{a} \\
8.343\end{array}$ & $\begin{array}{l}1.72 \\
\mathrm{a} \\
9.81\end{array}$ & $\begin{array}{l}2.04 \\
\mathrm{a} \\
8.94\end{array}$ & $\begin{array}{l}0.29 \\
0.04\end{array}$ & $\begin{array}{c}1.78 \\
\mathrm{a} \\
14.96\end{array}$ & $\begin{array}{c}1.56 \\
a b \\
11.38\end{array}$ & $\begin{array}{l}1.74 \mathrm{a} \\
11.09\end{array}$ & $1.17 \mathrm{~b}$ & $\begin{array}{c}0.012 \\
5 \\
1.56 \mathrm{E}\end{array}$ & $1.5 \mathrm{a}$ & $\begin{array}{l}1.42 \mathrm{a} \\
11.46\end{array}$ & $1.75 \mathrm{a}$ & 0.425 \\
\hline Linalool & $6-25.2$ & $2 \mathrm{a}$ & $a b$ & b & b & -02 & a & b & $\mathrm{ab}$ & 9 & a & b & b & $6.86 \mathrm{c}$ & -08 & $11.28 \mathrm{a}$ & a & $10.50 \mathrm{a}$ & 0.487 \\
\hline $\begin{array}{c}1- \\
\text { Octanol }\end{array}$ & 110 & $\begin{array}{l}16.75 \\
a\end{array}$ & $\begin{array}{l}16.1 \\
\mathrm{a}\end{array}$ & $\begin{array}{l}16.57 \\
\mathrm{a}\end{array}$ & $\begin{array}{l}13.96 \\
b\end{array}$ & $\begin{array}{r}1.53 \mathrm{E} \\
-04\end{array}$ & $\begin{array}{l}15.06 \\
\mathrm{a}\end{array}$ & $\begin{array}{l}16.31 \\
\mathrm{a}\end{array}$ & $\begin{array}{l}16.04 \\
\text { a }\end{array}$ & $\begin{array}{r}0.12 \\
5\end{array}$ & $\begin{array}{c}13.86 \\
\mathrm{a}\end{array}$ & $\begin{array}{c}16.49 \\
\mathrm{a}\end{array}$ & $\begin{array}{c}16.63 \\
\mathrm{a}\end{array}$ & $\begin{array}{c}21.75 \\
b\end{array}$ & $\begin{array}{l}4.25 \mathrm{E} \\
-05\end{array}$ & $14.96 \mathrm{a}$ & $\begin{array}{c}16.54 \\
\mathrm{a}\end{array}$ & $19.98 \mathrm{~b}$ & $\begin{array}{c}0.000 \\
797\end{array}$ \\
\hline $\begin{array}{c}\text { Ethyl } \\
\text { decanoat } \\
\text { e }\end{array}$ & 200 & $\begin{array}{l}14.31 \\
\mathrm{a}\end{array}$ & $\begin{array}{l}14.02 \\
2 \mathrm{a}\end{array}$ & $\begin{array}{l}20.04 \\
a b\end{array}$ & $\begin{array}{l}25.68 \\
b\end{array}$ & $\begin{array}{r}1.53 \mathrm{E} \\
-04\end{array}$ & $\begin{array}{l}17.93 \\
\mathrm{a}\end{array}$ & $\begin{array}{l}18.44 \\
\mathrm{a}\end{array}$ & $\begin{array}{l}19.72 \\
\mathrm{a}\end{array}$ & $\begin{array}{r}0.75 \\
7\end{array}$ & $\begin{array}{c}54.39 \\
\mathrm{a}\end{array}$ & $63.4 \mathrm{a}$ & $\begin{array}{c}65.13 \\
\mathrm{a}\end{array}$ & $\begin{array}{c}66.05 \\
a\end{array}$ & 0.315 & $63.96 \mathrm{a}$ & $\begin{array}{c}57.34 \\
a\end{array}$ & $65.38 \mathrm{a}$ & 0.396 \\
\hline 1- & & 13.18 & 13.62 & 13.44 & 11.95 & $1.28 \mathrm{E}$ & 12.59 & 13.13 & 13.33 & 0.49 & 6.21 & & & & 0.008 & & & & \\
\hline Nonanol & 50 & $\begin{array}{l}\mathrm{a} \\
0.15\end{array}$ & $\begin{array}{l}\mathrm{a} \\
0.127\end{array}$ & $\begin{array}{l}\mathrm{a} \\
0.149\end{array}$ & $\begin{array}{l}\mathrm{a} \\
0.162\end{array}$ & $\begin{array}{r}-01 \\
2.90 \mathrm{E}\end{array}$ & $\begin{array}{l}\mathrm{a} \\
0.149\end{array}$ & $\begin{array}{l}\mathrm{a} \\
0.139\end{array}$ & $\begin{array}{l}\mathrm{a} \\
0.153\end{array}$ & $\begin{array}{r}32 \\
0.68\end{array}$ & $\begin{array}{c}\mathrm{a} \\
0.447\end{array}$ & $5.72 \mathrm{a}$ & $5.6 \mathrm{ab}$ & $4.86 \mathrm{~b}$ & $\begin{array}{c}15 \\
6.63 \mathrm{E}\end{array}$ & $5.58 \mathrm{a}$ & $5.46 \mathrm{a}$ & $5.75 \mathrm{a}$ & 0.37 \\
\hline TDN & 2 & a & $\mathrm{a}$ & $\mathrm{a}$ & $\mathrm{a}$ & -01 & & $\mathrm{a}$ & a & 1 & $\mathrm{a}$ & $0.43 \mathrm{a}$ & $0.4 \mathrm{a}$ & $0.64 \mathrm{~b}$ & -06 & $0.44 \mathrm{a}$ & $0.45 \mathrm{a}$ & $0.46 \mathrm{a}$ & 0.764 \\
\hline
\end{tabular}




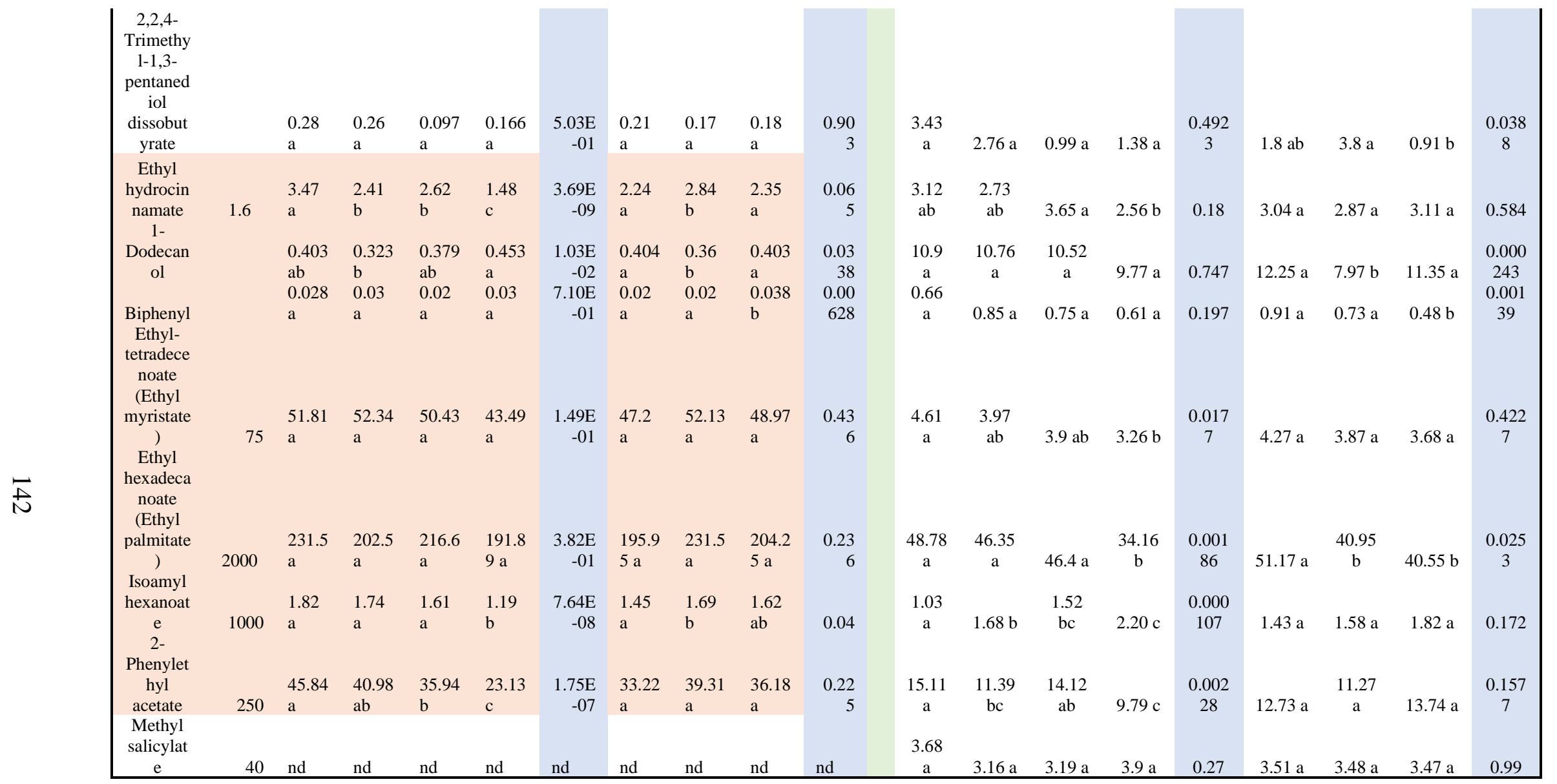




\section{Chapter 4: Rootstocks and Irrigation impact free and total volatiles in Chambourcin berries using a metabolomics-based approach.}

\section{Introduction:}

Grapevines, being a perennial plant, is faced with various environmental changes and stresses due to several conditions and thus, have to modulate its phenotypes to adapt to such changing environments (Young et al. 2016). The grape berries are the product or "sinkhole" of all such changes and stresses experienced by the plant during its growth and the plasticity demonstrated by the plant are manifested in berry composition and quality factors. The result of the interaction of grapevine genotype and the environment can modulate the composition of the final product of the plant and the berries (Anesi et al. 2015). Therefore, in this study, it is imperative to not only study the effect of rootstock and irrigation in wine volatiles but equally important to study the berry volatiles.

Rootstocks have been extensively used in vineyards since the second half of the $19^{\text {th }}$ century because of the phylloxera invasion in Europe. Although primarily used to confer pest resistance, rootstocks play an important role in vine adaptation to various environmental conditions such as drought and salinity (Serra et al. 2014; Zhang et al. 2016). Rootstocks are the link between soil and the scion and thus, have a substantial influence on the physiology of the scion (Tramontini et al. 2013) as a consequence of genetic differences in root density, and architecture. Through their influence on vegetative growth, gas-exchange, water status, or nutrient uptake, rootstocks can influence yield and berry composition (Jones et al. 2009a; Koundouras et al. 2009; Nelson et al. 2015; Koundouras et al. 2008). By affecting berry size and fruit chemistry 
parameters such as sugar content, organic acids, anthocyanins, and amino acids, rootstocks can impact the berry and wine volatiles, which are important quality determinant (Jones et al. 2009a; Harbertson and Keller 2011; Olarte Mantilla et al. 2018; Berdeja et al. 2014). Rootstocks have been found to impact the concentrations of volatile compounds and their aroma precursors in Cabernet Sauvignon grapes (Wang et al. 2019; Koundouras et al. 2009). Rootstocks also have a positive impact on wine volatiles in Shiraz and Merlot (Olarte Mantilla et al. 2018; Carrasco-Quiroz et al. 2020). While research has been done to study the impact of various rootstocks on the berry composition of V. vinifera, (Koundouras et al. 2009; Olarte Mantilla et al. 2018; Berdeja et al. 2014), information about the rootstocks on berry volatiles and their precursors are scarce on the interspecific hybrid grapes and wines. This is likely because of the more limited planting of interspecific hybrids and the fact that their phylloxera tolerance generally allows them to be grown own-rooted, though there are numerous other reason why grafting may be beneficial and thus need to be studied (Thomas et al. 2017; Main et al. 2002).

On the other hand, plant water status has a major influence on the physiological characteristics of vines (photosynthesis, growth, nutrient supply) and fruit and wine quality (Tramontini et al. 2013). The effects of water stress on grapevine (Vitis vinifera) metabolism, vegetative development, productivity or yield, and berry composition have been explored for many combinations of rootstocks, cultivars, and climatic conditions (Berdeja et al. 2014; Berdeja et al. 2015; Harbertson and Keller 2011; Keller et al. 2012; Koundouras et al. 2009; Romero et al. 2019). Moderate water stress mediated through deficit irrigation has been found to have a positive impact on berry and wine quality by 
increasing the concentration of desired volatiles such as norisoprenoids (Vivian et al. 2018; Reynolds et al. 2007; Romero et al. 2019; Qian et al. 2009). However, the extent to which berry aroma compounds are affected by water stress and rootstocks in hybrid grapevines has seldom been assessed. The intensity of water stress and its timing of occurrence over the grape growing period is of paramount importance. Water stress imposed during pre-veraison stages induces major and long term metabolic changes that remain even after rewatering (Koundouras et al. 2009; Koundouras et al. 2008).

The concentration and composition of these compounds change during berry development and are directly and indirectly impacted by various environmental factors, genetic makeup, management practices, and developmental stages. Veraison is characterized by the accumulation of sugars, anthocyanins, pigments, and additional phenols. After veraison, high concentrations of glucose and fructose accumulate in the berries along with a decrease in organic acid levels, especially malic acid, and berries swell and become softer due to water uptake, seeds mature, and $\mathrm{pH}$ increases due to the accumulation of cations (Coombe et al. 1987; CooMbe and McCarthy 2000; Ristic and Iland 2005). Berry ripening is also accompanied by the synthesis of many aroma compounds (terpenes, norisoprenoids, esters, and thiols) and their precursors (Kennedy 2002; Kalua and Boss 2010; Yuan and Qian 2016). Berry chemistry during both phases of growth is highly influenced by many factors including cultivar, rootstock, shading, climate, pest and disease pressures, and soil nutrients (Barnuud et al. 2014; Galletto et al. 2014; Main et al. 2002).

Aroma is an important quality aspect that determines the consumer acceptance of the grapes and wine and is derived from grapes, the fermentation process, and wine 
storage (Ebeler 2001; Ebeler and Thorngate 2009; Rapp and Mandery 1986). Grapederived aromas can be free volatiles that are present in skin and pulp as well as glycosidic bound volatiles in grapes that are released by enzymatic/catalytic reactions (Ferreira and Lopez 2019). The varietal difference in the proportion of the glycosidic aroma compounds has been observed due to the genetic variation in aroma biosynthesis genes between grape varieties as well as due to the type and amount of aglycone attached with the bound volatiles. The interaction between the individual aroma compounds and matrix also shapes the aroma profile of grapes and wines. While varietal differences in aroma have been studied in grapevines, the variation in aroma due to changes in the root system genetics of the grapevine on an identical scion has not been explored, even less so in hybrid grapes. Monoterpenes, C13-norisoprenoids, alcohols, esters, and benzyl alcohol are known to be important volatiles in vinifera and hybrid grapes and wines (Slegers et al. 2015; Slegers et al. 2017; Sun et al. 2011b). Although C13-norisoprenoid have been known to be derived from carotenoid degradation by the action of carotenoid cleavage dioxygenase (CCD) enzymes and constitute an important part of volatile compounds in nonfloral grapes such as Cabernet Sauvignon (Kalua and Boss 2010), Syrah and Pinot noir (Yuan and Qian 2016), $\beta$-Damascenone formation and development are not clear as there is conflicting information about its development in berries, suggesting that there might be alternative production pathways. With the sensory threshold of $0.05 \mathrm{ug} / \mathrm{L}$ in hydroalcoholic solution and high odor activity, $\beta$-Damascenone is a potent aroma compound in wines contributing honey, tobacco, and floral aroma (Pineau et al. 2007; Mendes-Pinto 2009; Sefton et al. 2011; Kotseridis et al. 1999). This compound is also 
known to have an indirect impact on grapes and wine aroma by enhancing fruity aromas and masking herbaceous aromas (Pineau et al. 2007).

As each grape cultivar has a different volatile profile, looking at the limited few known metabolites in new and uncharacterized grape cultivar such as Chambourcin might pose a risk of missing many other important volatile compounds that are important for the survival of these plants in cold winters and hot and humid summers. Looking through a holistic approach will help us understand how the volatile composition comes about and suggest ways to manipulate the production of volatile compounds to produce high-quality wines. Therefore, to understand the impact of grafting rootstocks in hybrid cultivar Chambourcin, we opted for an inclusive metabolomics-based approach so that no important metabolites are missed. Also, most of the hybrid grapes are grown own-rooted, however, since rootstocks can impart scions ability to survive in unfavorable environmental situations, we investigated how rootstocks in a hybrid scion can impact the berry composition, organic acids and free and bound volatiles in grapes at different developmental stages.

\section{Materials and Methods:}

\subsection{Experimental design and sampling and sample processing:}

This study was conducted in the same experimental vineyard used in Chapter 3 for wine analysis (please refer to section 2.2 in Chapter 3). Berries were collected in both 2017 and 2018 using two time points; one at veraison (when $50 \%$ of the berries start to change color) and other at harvest from 71 plants per sampling for berry chemistry $((\mathrm{pH}$, titratable acid, soluble solids, organic acids) and free and bound volatile analysis $(71=4$ 
rootstock combinations x 3 replicates/vine x 3 irrigation treatments x 2

replicates/irrigation treatment). One of the vines in row 10 reverted back to its SO4 rootstock (rootstock vine), therefore, that vine was not included in analysis. At each timepoint, we sampled representative 2-3 berry clusters and flash froze in liquid nitrogen to prevent any enzymatic reactions and to capture the metabolic state of the grapevine when attached to the vine. The frozen grapes were transported to the laboratory in dry ice and stored at $-80^{\circ} \mathrm{C}$ until analyzed. The grapes were then analyzed for free and bound volatiles.

\subsection{Extraction of free and total volatiles from berries:}

Extraction of free and total volatiles from berries was done using the same protocol as in Chapter 2, section 3.2 and 3.3. For free volatile analysis, $5 \mathrm{ml}$ of the extract was taken in a $15 \mathrm{ml}$ SPME vial and $50 \mu \mathrm{l}$ of internal standards were added to make a final concentration of $0.5 \mathrm{mg} / \mathrm{L}(50 \mathrm{ppb}) 2$-Octanol and $1 \mathrm{mg} / \mathrm{L}(100 \mathrm{ppb})$ of 4methyl-2-pentanol, followed by the addition of $2 \mathrm{gm} \mathrm{NaCl}$ per vial.

For total volatiles (free plus bound volatiles), a method modified from Hampel (2014) was adopted (Hampel et al. 2014). A stock Rapidase enzyme solution (250,000 mg/L) was freshly prepared using $2.5 \mathrm{~g}$ of Rapidase in $10 \mathrm{ml}$ of DI water. In a $15 \mathrm{ml}$ glass vial, $5 \mathrm{ml}$ aliquots of the supernatant were spiked with $20 \mu \mathrm{l}$ of the Rapidase enzyme solution to yield a final enzyme concentration of $1000 \mathrm{ppm}$. The internal standard cocktail used was yielded a final sample concentration of $0.5 \mathrm{mg} / \mathrm{L}(50 \mathrm{ppb}) 2-$-Octanol and $1 \mathrm{mg} / \mathrm{L}$ (100 ppb) of 4-methyl-2-pentanol. The glass vials were sealed and incubated in a $45^{\circ} \mathrm{C}$ water bath for four hours. The vials were then cooled to ambient temperature in a $25^{\circ} \mathrm{C}$ water bath for 10 minutes to prevent headspace volatilization. $2 \mathrm{~g}$ of $\mathrm{NaCl}$ was added to 
the sample vial to inactivate the Rapidase enzyme. The samples were then processed for volatile aroma compounds using the HS-SPME-GC-MS/MS method outlined below.

\subsection{Head Space Solid Phase Microextraction Gas Chromatography-Mass Spectrometry:}

The HS-SPME-GCMS/MS method used in Chapter 3, section 2.5 was used. Berry volatiles data processing using untargeted metabolomics analysis:

Data processing was done for free volatiles and total volatiles separately. However, for each analysis, data from different timepoints and years were analyzed together. Data were analyzed using XCMS Online (Tautenhahn, Patti et al. 2012, Gowda, Ivanisevic et al. 2014), available at https://xcmsonline.scripps.edu/ using the same protocol as in Chapter 3. Results were downloaded from the XCMS online on March 11-12, 2020. The extracted features (intensity of a given $\mathrm{m} / \mathrm{z}$ at a certain time in minutes) were used for further analysis in defining treatment differences. The features $(\mathrm{M}(\mathrm{m} / \mathrm{z}) \mathrm{T}(\mathrm{time}$ in minutes)) with more than $50 \%$ missing values were removed and remaining missing values were replaced by a small value (half of the minimum positive value in the original data). Data filtering using interquartile range was then performed to remove features arising from the baseline noises and to remove features that do not change throughout the treatments that are unlikely to be used for further analysis. Then, the data was logtransformed and auto-scaled (mean-centered and divided by the standard deviation of each variable) and then used for further statistical analysis. The autoscaling method was used to remove any variation comprised during analysis (such as a loss of instrumental sensitivity) of an original MS peaks list. Principal component analysis (PCA) applied after autoscaling was evaluated for sample discrimination. 


\subsection{Statistical analysis:}

The data from each analysis (free or total volatiles) were analyzed using R v3.5.0 (Team 2019). The principal component analysis was performed with the features $(\mathrm{M}(\mathrm{m} / \mathrm{z}) \mathrm{T}$ (time in minutes)). PCA is especially useful in a data with a large number of correlated variables as it has the capacity to reduce the complexity of a huge dataset by extrapolating important information and displaying it as a set of new independent variables called principal components that collectively explain most of the variability in the original dataset. Basically, it is important to know the similarity pattern of the observations or variables. We performed PCA on free and total volatiles from both years and timepoints separately to identify features that are significantly different from the background noise for free and total volatiles. The top 20 PCs were used in a linear model as new traits to understand the effect of the experimental design on the metabolome. The model accounted for variation in the block (position in the vineyard), irrigation (Full, RDI, None), rootstock (1103P, 3309C, SO4, and own-rooted), year (2017 and 2018) and phenology (timepoints of sampling). The linear model was calculated using the ' $\mathrm{lm}$ ' function in $\mathrm{R}$ and the percentage of total variation explained by each PC was calculated using the ANOVA function, and only factors which explained a significant portion of variance $(\mathrm{p}<0.05)$ were visualized using ggplot 2 in $R$. When irrigation or rootstock explained a significant portion of variance, boxplots were constructed. Principal components (PCs) that varied significantly by phenology, year, rootstocks, and irrigation were then analyzed to extract specific features $(\mathrm{M}(\mathrm{m} / \mathrm{z}) \mathrm{T}$ (time in minutes)) responding to the treatments. Only the features that loaded above or below the $95 \%$ confidence interval ( $\mathrm{z}$ score $=1.96$ ) were fit independently with the same linear model. The cutoff of 
1.96 standard deviations from mean was chosen as it represents the area beyond the $95 \%$ confidence interval. The main effect of rootstock and irrigation was then determined using pairwise posthoc comparisons of means. The percentage of total variation explained by each metabolite was calculated using the ANOVA function, and only factors which explained a significant portion of variance $(\mathrm{p}<0.05)$ were visualized using ggplot2 in R. Estimated p-values for each factor in the models were corrected for multiple tests (within phenotype) using FDR correction. When appropriate, posthoc mean comparisons were computed using the package 'emmeans'.

\subsection{Identification and confirmation of the compounds:}

After the identification of significant features, the compounds represented by the features were tentatively identified using the NIST MS Search v2.2, NIST 14 Mass Spectral Library database (Scientific Instrument Services, Ringoes, NJ, USA). Linear retention indices (RI) were calculated using Kovats' equation from a sequence of linear hydrocarbons from $\mathrm{C} 7$ to $\mathrm{C} 30$. Possible matches for volatile compounds were first identified by comparison of the mass spectral data within the NIST library and then verified as a valid prospect based on RI data.

The confirmation and quantitation of volatile compounds were achieved using calibration curves for each standard at five different concentration levels in cases where standards are available. For compounds whose standards were not available, a semi-quantitative analysis was done by assuming a response factor equal to one to 2-Octanol IS equivalents. Agilent MassHunter Quantitative Analysis (for QQQ) B.07.01 (Agilent Technologies) was used. Each standard was prepared in model wine solution $(8 \mathrm{~g} / \mathrm{L}$ of tartaric acid, dissolved in $13 \%$ ethanol solution (v/v), at $\mathrm{pH} 3.2$, adjusted with $\mathrm{NaOH})$. 


\subsection{Predictive nature of features:}

We examined the predictability of the features between phenology and years for free and total volatiles. Pairwise correlations were tested using Pearson's correlation and pvalues were corrected for multiple testing using FDR at a significance level of 0.05 . Pearson's correlation was performed using the 'psych' package in R v3.5.0. Two different categories of correlation analysis were performed: one to predict harvest metabolites using veraison metabolite features and another to predict if the features are predictable year wise. This was performed for free and total volatiles separately. All the significant correlations were FDR adjusted and only the correlations that are significant between different phenology timepoints, as well as years, were used to do downstream analysis. Later, only the significant correlations that are between features with the same retention times but with different timepoints or years were considered.

\section{Results:}

\subsection{Feature Identification:}

To characterize the free and total (i.e. free plus glycosidically bound) volatiles in the berries, as well as their changes within a year and across years, we sampled berries at two timepoints (veraison and harvest) for two years (2017 and 2018). All GC-MS data for both years and timepoints ( 71 vines $* 2$ years $* 2$ timepoints $* 2$ reps $=568$ runs) were processed as a single job in XCMS online for free volatiles and total volatiles separately to allow comparison across sampling periods.

We identified 271 and 259 metabolic features $(\mathrm{M}(\mathrm{m} / \mathrm{z}) \mathrm{T}$ (time in minutes)) for free and total volatiles respectively (Table S1 and S2). Heatmaps using the top 25 features 
demonstrated differences between rootstocks and irrigation for both free and total volatiles (Figure 4.1). Principal components analysis (PCA) using those features indicated that both free and total volatiles did not have clear grouping or separation based on rootstocks as well as irrigation (Figure 4.2). Interestingly, the majority of the separation demonstrated by PC1 and PC2, for both free and total volatiles, were by state/phenology (both sampling time and year). Veraison at both years was found to have smaller spread than the harvest for both years, indicating that at veraison volatile makeup is more similar in comparison to that of harvest. Using linear modeling of the top 20 PCs, we were able to identify the 8 PCs that contributed significantly due to rootstock differences and irrigation regimes, and so then we used those PCs to identify important metabolites that are significantly different due to those factors.

\subsection{Free volatiles:}

Free volatiles that is present in the grapes and released during grape crushing were analyzed using principal components analysis. PC1 and PC2 explained 29.2 and $16.3 \%$ of the variance in the data for free volatiles (Figure 4.2). To examine the variation in free volatiles due to different factors and treatments in our experimental design, we used the first 20 principal components to build a linear model that included rootstock (own-rooted, 'SO4', '1103P' and '3309C'), irrigation (none, RDI, Full), year of sampling (2017, 2018), block (position in the vineyard), and phenology (timepoints at which berries were sampled). The interactions between different factors were not involved in the model as our aim here was to simply decipher the contribution of each factor in the already complex experiment and additionally sample size if broken into complex subgroups, would be exceedingly small. The first 20 PCs explained a total of $85.36 \%$ of the variation 
in free volatiles. We used linear modeling of the top 20 PCs to explain percent variation contributed by each factor and then used each significant PC to detect metabolites that are most significant in each PC and modeled each metabolite identified using the same linear model.

For the first principal component (PC1), the primary source of variation for free volatiles described by our model was the year $(43.77 \%)$ followed by phenology $(42.53 \%)$ (Figure 4.3). Phenology and year explained $35.91 \%$ and $17.47 \%$ variation respectively in free volatiles by PC2 (Table S3). Phenology explained a significant portion of the variation in berry free volatiles for 10 PCs and year was a significant factor of variation for free volatiles for 9 PCs (Figure 4.3). Rootstocks and irrigation did not contribute significantly to PC1 whereas phenology, block, and year contributed significantly to PC1 (Figure 4.3). PC2 explained minute but significant variation due to rootstock (1.52\%).

For rootstocks, significant variation was explained by 8 PCs where out of 8,3 PCs PC8, PC13, and PC17 explained the most variation (Figure 4.3). PC8 explained a significant rootstock effect, explaining $4.62 \%$ of the variation (Table S3). Similarly, PC13 and PC17 explained 3.54\% and 3.76\% of the variation (Figure 4.3).

Similarly, for irrigation, significant variation was explained by 4 PCs including PC8 (0.97\%), PC11 (2.03\%), PC14 (2.13\%) and PC20 (2.19\%) (Figure 4.3). Of the 42 significant relationships $(\mathrm{p}<0.05)$ identified for PCs 1 to 20,8 explained a significant portion of free volatiles variation due to rootstock, and four for irrigation. (Fig 4.3). 


\subsubsection{Impact of phenology and year on free volatiles:}

To identify the metabolites that were mostly driving the variation due to phenology and year, we selected the first two PCs (PC1 and PC2) that explained the major amount of variation due to phenology and year and used the features that loaded more than 1.96 standard deviation from mean to fit into the same model.

We identified 13 features that loaded significantly in PC1. All 13 features explained significant variation due to phenology whereas 9 features explained significant variation due to year followed by 7 features due to block (Figure 4.4A). Interestingly, the features with retention time (RT) 5.68 found to be the most reoccurring feature (Figure 4.4A). Using the NIST library, the features with retention time (RT) 5.68 is identified to be Hexanal (Figure 4.4B).

Similarly, we found 31 features loaded significantly in PC2 (Figure 4.4C). All 31 features were significant due to phenology, followed by 29 for block and year (Figure 4.4C). Rootstock was a significant factor for 12 features in PC2. Using the NIST library, the features with retention time (RT) 5.68 and RT 8.64 were identified to be Hexanal and 2-Hexenal, (E) (Figure 4.4D). Both Hexenal and 2-Hexenal, (E ) are known to have a green-leaf note, cut grass aromas, mostly found in green unripe berries, which is not surprising because as veraison berries are sample the berries just started to change color and have a more green, unripe aroma. 


\subsubsection{Impact of rootstock on free volatiles:}

We used the same approach as we did for years to identify the metabolites that had significant variation due to rootstock. We found PC8, PC17, and PC13 significantly explained most of the variation due to rootstock (Figure 4.5).

PC8 explained $4.62 \%$ of the variation in free volatiles due to rootstocks (Table S3) and demonstrated that berries from grafted vines had higher intensity of free volatiles than own-rooted berries. We identified 12 features contributing most to the PC8 (Figure 4.5A) out of which four were causing significant variation due to rootstocks (Fig 4.5A). Posthoc comparisons of those metabolites demonstrated that feature M41T22.29 ((M(m/z) T(time in minutes)) was significantly different from own-rooted and 3309C. Features M69T23.47, M190T23.47, and M122T23.47 were higher in own-rooted berries than grafted berries (only feature M69T23.47 shown in Fig 4.5B). The features with RT 23.47

were identified to be $\beta$-Damascenone using the NIST database and in-house database and then confirmed using authentic standard. This compound was found to be higher in concentration in berries from own-rooted vines than grafted vines (Figure 4.5B).

Rootstocks explained $3.76 \%$ of the variation in free volatiles in PC17 (Figure 4.5C). There were significant differences between free volatiles in 1103P and 3309C and own-rooted as indicated by PC17. We identified 23 significant metabolites in PC17. Among 23 metabolites, 8 metabolites contributed most to the variation due to rootstocks. However, post hoc comparisons using FDR corrections only identified 4 significant features due to rootstocks. For all the four features (M95T22.29, M82T22.29, M41T22.29, and M57T15.83), own-rooted berries had significantly lower of the metabolite than berries from the grafted plant (only one significant metabolite shown in 
Figure 4.5D). The compound with RT 22.29 was tentatively identified to be citronellol based on the NIST library (Figure 4.5D).

PC13 also explained significant variation in free volatiles due to rootstocks (Fig 4.5E). We found 13 significant features in PC13 among which four features (M69T23.47, M190T23.47, M122T23.47, and M106T16.68) were found to be contributing significant variation due to rootstock from the post hoc mean analysis (only one significant metabolite shown in Figure 4.5F). Based on the NIST library, RT 23.47 was identified to be $\beta$-Damascenone and confirmed using an authentic standard. $\beta$-Damascenone was confirmed and quantified using authentic standards. The feature with RT 16.68 was tentatively identified to be ethyl nonanoate and features with RT 15.83 could not be identified (data not shown).

\subsubsection{Impact of irrigation on free volatiles:}

PC8, PC11, PC14 and PC20 explained significant variation in free volatiles due to irrigation (Figure 4.6), contributing $0.97 \%, 2.03 \%, 2.13 \%$ and $2.19 \%$ respectively. We identified 12 significant features in PC8 and three of those features (M122T23.47, M190T23.47, and M69T23.47) explained significant variation due to irrigation (Figure 4.5A). None was found to be significantly different from the RDI. All three features have the same retention time and represent the same compound, which is identified to be $\beta$ Damascenone using the NIST library and later confirmed using authentic standards.

PC11 demonstrated differences in free volatiles between full and RDI. We found 14 significant features (Fig 4.6A). Among the 14 features, 5 of them indicated significant contribution due to irrigation, and only 4 of them were statistically significant according 
to the posthoc mean comparisons. Features M91T23.47, M77T23.47 (identified and confirmed to be $\beta$-Damascenone ) showed significant differences between none and RDI and features M81T9.1, M82T9.17 (tentatively identified to be ethyl hexanoate from NIST) showed significant differences between Full and RDI and None and RDI, showing RDI had a different effect on the volatiles than full or none.

We found 20 features that contributed most to PC14 (Figure 4.6C) and using the same linear model on each of these metabolites, we found that 8 of them are significant from posthoc means comparisons. All of the significant metabolites had the same retention time of 23.47 (Fig 4.8C) which indicated that the same compound, identified to be $\beta$ Damascenone was impacting the differences between none and RDI. In PC20, three features out of 13 influential features were found to be significantly different due to rootstocks, however, with post hoc mean comparisons, only two of them are significant (M175T14.27, and M80T8.46). All the PCs and significant metabolites indicated RDI treated berries having different free volatiles in comparison to other treatments (one of the metabolites shown in figure 4.6D).

\subsection{Total volatiles:}

Total volatiles comprises the volatiles present freely in the grapes as well as volatiles released from precursors due to crushing and glucosidase enzyme treatment (free and bound). PCA analysis of 259 metabolic features of total volatiles including both years and phenology explained $35.48 \%$ and $12.94 \%$ variation due to PC1 and PC2 (Figure 4.7). The factors driving the separation of the total volatiles by PC1 and PC2 are years and phenology (Fig 4.7) which is similar to free volatiles. There was a separation between years for harvest, however, veraison of both years overlapped (Figure 4.2C and D) 
indicating that total volatiles at veraison was similar in both years and might be driven due to changes in physiology and development, not by yearly variation. PC1 to PC20 explained a total of $85.68 \%$ of the variation and were used in the linear model to understand the contribution by each factor. Six PCs explained significant variation due to rootstocks and three PCs explained significant variation due to irrigation. Phenology was significantly explained by 5 PCs, the block was significantly explained by 11 PCs and year by 8 PCs (Figure 4.7). Phenology explained most of the variation in PC1 (63.96\%) and PC2 (18.13\%).

For the first principal component (PC1), the primary source of variation for total volatiles described by our model was phenology $(63.96 \%)$ followed by the year $(20.91 \%)$ (Figure 4.7). PC4 explained a significant rootstock effect, explaining $1.37 \%$ of the variation and $21.87 \%$ variation due to year (Figure 4.7). Similarly, PC7 and PC8 explained $8.04 \%$ and $4.03 \%$ of the variation due to rootstock (Table S4). Similarly, PC14 and PC18 explained $2.64 \%$ and $1.88 \%$ of phenotypic variation due to rootstocks. Significant variation due to irrigation was explained by PC12 (2.99\%), PC15 (2.84\%), and PC16 (3.46\%) (Figure 4.7).

\subsubsection{Impact of phenology and year on total volatiles:}

To identify the metabolites that are mostly driving the variation due to phenology and year, we used PC1 and PC2 that explained the largest amount of variation due to phenology and year and extracted the features that loaded more than 1.96 standard deviation from the mean (beyond 95\% confidence interval) to fit into the same model. We identified 17 significant metabolites in PC1 (Figure 4.8A). All 17 features explained significant variation due to phenology and block, followed by 14 for a year. Irrigation did 
not contribute significantly to PC1, only one feature was explained significantly by rootstock (Figure 4.8A). Similarly, in PC2, 14 features were significant due to phenology and year (Figure not shown). The compounds were identified to be 2-Hexenal,(E)-(RT 8.8), 3-Hexenal (RT 6.87), mostly having grassy aroma (one of the features shown in Figure 4.8B). Compounds cannot be identified for some features as positive relation of features to compound spectra in the database was not possible for many features due to limitations of the database, extraneous signals associated with solid-phase microextraction, potential co-elution causing a combined spectra or poor spectral differentiation within certain common classes of compounds.

\subsubsection{Impact of rootstocks on total volatiles:}

To identify the metabolites explaining significant variation by rootstock, we used the PCs that explained the highest variation due to rootstock. For PC7 in which the primary source of variation for total volatiles described by our model was rootstock (8.04\%), we identified significant 17 metabolite features (Figure 4.9 C). Total volatiles from own-rooted berries was lower than rootstocks as shown in PC7 (one of the features shown in figure 4.9D). Only 7 features were explained significantly by rootstock.

For the PC8, the primary source of variation for total volatiles described by our model was rootstock $(4.03 \%)$ and there is significant variation between rootstocks (Figure 4.9 E). 7 features were significant due to rootstocks.

In PC4, only two features (M55T16.95 and M120T22.49) were significant for rootstocks in which own-rooted vines are shown to have lower total volatiles than grafted vines (Fig 4.9A and B). Most compounds that are impacted by rootstocks were 
tentatively identified to be $\beta$-Damascenone (RT 23.44), Methyl salicylate (RT 22.49), and Ethyl Nonanoate (RT 16.95). Among the identified compounds, $\beta$-Damascenone was confirmed and quantified.

\subsubsection{Impact of irrigation on total volatiles:}

PC12, PC15, and PC16 explained significant variation due to irrigation for total volatiles (Figure 4.10), contributing $2.99 \%, 2.84 \%$ and $3.46 \%$ of the variation, respectively. We identified 16 significant features in PC12, only three of them (M81T9.04, M82T9.06, and M147T11.96) explained significant differences due to irrigation (Fig 4.10A). Features M81T9.04 and M82T9.06 are significantly based on post hoc mean comparisons, showing differences between none and RDI, and full and none with RDI respectively.

PC15 showed none was significantly different from full and RDI. Among the 20 features that most explained the variation in PC15, only five of them are significant at pvalue $<0.05$ (Figure 4.10B) and only 3 of them significant when tested for significant posthoc mean comparisons(M81T9.04, M82T9.06, and M82T26.48).

PC16 showed that full irrigation to be different from none and RDI for total volatiles. Among the 15 features that explained most of the variation in PC16 (Figure 4.10C), only 2 of them were significant (M70T10.99, M55T10.98). The compounds that are impacted by irrigation were tentatively identified to be 1-Dodecanol (RT 26.48).

\subsection{Predictive nature of features:}

Correlation between metabolite features with the same RT was performed to estimate the predictability of harvest based on the veraison for both free and total volatiles 
separately and to see if metabolites can be predicted based on the year. By correlating veraison features with harvest features, we found 31 significant correlations with Pearson's correlation coefficient $>0.3$ and FDR corrected $p$-value $<0.05$ between 2017 and 2018 for free volatiles (Table 4.4). Also, 15 features were predictive for free volatiles between veraison and harvest for 2017 and 2018 (Table 4.3).

For total volatiles, there were no significant correlations $(r>0.3$, $p$-value $<0.05)$ that showed the predictability of total volatiles in 2017 and 2018. Only three features are predictive in harvest based on veraison in 2017. In 2018, 14 significant correlations between veraison and harvest for total volatiles. Identification of compounds from the features by comparing mass spectra with NIST library was performed and we found that 2-Hexen-1-ol,(E )- (RT 13.37) and 2-Octanone (RT 10.59) were predictable between veraison and harvest in 2018 for free volatiles (Table 4.3). In 2017, $\beta$-Damascenone (RT 23.47), 2-Hexenal, (E)- (RT 8.9), and Phenylethyl alcohol (RT 25.38) can be predicted in harvest from veraison as free volatiles. For total volatiles, Ethyl hexanoate (RT 9.19) was predictable in 2017 between veraison and harvest (Table 4.4). In 2018, ethyl nonanoate (RT 16.73), linalool (RT 17.24), and methyl salicylate (RT 22.49) were predictive total volatiles between veraison and harvest (Table 4.3). Compounds such as 1Hexanol (RT 12.35), 2-Hexen-1-ol, (E )-, 2-Hexen-1-ol, (Z)-, 1-Dodecanol, Ethyl 9hexadecenoate, $\beta$-Damascenone, Phenylethyl alcohol, ethyl hexanoate were predictive between 2017 and 2018 (Table 4.4).

\section{5 $\beta$-Damascenone concentration differences due to rootstocks and irrigation:}

Since $\beta$-Damascenone was identified as a compound that was impacted by rootstocks as well as irrigation treatments in our experiment, we quantified this compound semi- 
quantitatively using the calibration curve of the $\beta$-Damascenone pure standard (Table 4.1). The concentration of $\beta$-Damascenone in Chambourcin berries was found in the range of 5.34-7.98 $\mu \mathrm{g} / \mathrm{L}$ at veraison and in the range of $4.23-11.53 \mu \mathrm{g} / \mathrm{L}$ at harvest. Free $\beta$-Damascenone was found to be significantly different between rootstocks at harvest in 2017 and 2018 (Table 4.1) and veraison in 2018, but not in 2017. Berries from ownrooted vines contained significantly higher free $\beta$-Damascenone than other rootstocks at both veraison and harvest in both years. Irrigation has been found to significantly impact free $\beta$-Damascenone concentrations at harvest 2018 (p-value 0.035) and veraison 2017 (p-value 0.0683). Berries from RDI-treated vines had a significantly higher concentration of $\beta$-Damascenone than other irrigation treatments (Table 4.1). The concentration of $\beta$ Damascenone was higher in veraison in 2017 (5.34-6.36 $\mu \mathrm{g} / \mathrm{L})$ compared to harvest (5.57-7.98 $\mu \mathrm{g} / \mathrm{L})$, whereas, in 2018, $\beta$-Damascenone concentration was higher at harvest $(7.89-11.53 \mu \mathrm{g} / \mathrm{L})$ in comparison to veraison in $2018(5.57-7.98 \mu \mathrm{g} / \mathrm{L})$ (Table 4.1).

We also measured total (free and bound combined) $\beta$-Damascenone at veraison and harvest in both 2017 and 2018. The concentration of total $\beta$-Damascenone was observed to range from 11.19 to $18.21 \mathrm{ug} / \mathrm{L}$ at veraison and $9.42-17.6 \mathrm{ug} / \mathrm{L}$ at harvest. Rootstock had a significant effect on total $\beta$-Damascenone at veraison 2018 (p-value 0.0029) and harvest 2018 (p-value 3.8e-05). Irrigation did not impact total $\beta$-Damascenone concentrations in 2018 and 2017, however, some significance was observed at harvest 2017 (p-value 0.089). Own-rooted berries had been found to contain higher concentrations of total $\beta$ Damascenone in comparison to other rootstocks. Berries from treated RDI vines had higher total $\beta$-Damascenone at harvest 2017, but not in 2018. 
We also examined the bound fraction of the volatiles $\beta$-Damascenone (total minus free) that are mostly derived from the aroma precursors due to enzymatic hydrolysis (Table 4.1). Bound $\beta$-Damascenone were found to be almost significant at veraison in 2018 (p-value 0.069 ) between rootstocks, indicating own and 3309C had higher concentrations of bound $\beta$-Damascenone (10.23 and $10.25 \mathrm{ug} / \mathrm{L}$ respectively) than other rootstocks. Similarly, between three irrigation regimes, RDI had significantly higher bound $\beta$-Damascenone (14.51 ug/L) than others in harvest 2017 (p-value 0.0454) (Table 4.1). While in 2017, the bound fraction of $\beta$-Damascenone had increased concentrations in 2017, however, in 2018, the concentrations were almost equal and sometimes lower than that of free volatiles.

\subsection{Total Methyl Salicylate is impacted by rootstocks:}

Total Methyl Salicylate (RT 22.49) has been identified to be impacted by rootstocks (Fig 4.11). We semi-quantitatively quantified this compound in free and bound form and found a significant impact of rootstock at veraison and harvest in 2018 (Table 4.2).

Veraison at both years contained higher concentrations of methyl salicylate in comparison to harvest. Free methyl salicylate was found to be significantly different between rootstocks, with 1103P having the highest concentration. Similarly, berries from $1103 \mathrm{P}$ vines have higher concentrations of total methyl salicylate in comparison to other rootstocks and own-rooted. Own-rooted had had the lowest concentrations of total methyl salicylate (Table 4.2).

\section{Discussion:}

As grapes provide the basis for many wine aromas and flavors, the composition of berries during berry development and the factors affecting their composition is of much 
interest. Most grape aroma compounds are present in berries either as free volatiles, which may contribute directly to the aroma or as non-volatile bound volatiles or aroma precursors, which represent the aroma potential as they can release free volatiles due to acid or enzyme hydrolysis during the process of winemaking. The flavor and aroma characterization on grapes and wines typically utilize the 'targeted' approach to identify compounds that contribute to the flavor attributes of grapes and wines (Escudero et al. 2007; Ferreira and Lopez 2019; Ferreira et al. 2000; Vrhovsek et al. 2014). While this approach has advanced our understanding of the compounds that contribute mostly to the aroma of grapes or the compounds present in higher concentration within wellcharacterized Vitis vinifera grapes, metabolomics data is potentially overlooked as only the compounds that directly produce a sensory response would be included, ignoring any modulating activity and potential interactions between compounds and matrix (Polaskova et al. 2008; Robinson et al. 2009). Applying comprehensive untargeted profiling analytical approaches provides new opportunities to characterize as many compounds as possible or at least those with certain properties, providing a complementary approach to targeted methods, ultimately to generate novel information about the aroma profile of grapes and wines. This approach is especially useful for interspecific cultivars such as Chambourcin that have had fewer studies conducted about its aroma profile. Instead of quantifying the selected number of compounds, we identified 271 and 259 metabolic features or putative compounds for free and total volatiles respectively. Using univariate and multivariate approaches such as linear modeling and PCA, we were able to model these features to identify the features that are most significantly impacted by the factors in our experiment. PCA scores plot did not show clear separation based on rootstocks or 
irrigation. Partial separation in the PCA scores plot could indicate that there are distinct patterns in at least some metabolite levels that distinguish between the groups, however, the patterns may be weak, or confounded by strong variations in metabolites due to other factors associated with individual variabilities. Therefore, we looked at other factors of variation in our linear model to capture the variation from other sources besides rootstocks and irrigation.

Phenology was found to be the most significant factor impacting the berry volatiles (both free and total) explaining a major portion of variation (43.77\% and $63.93 \%$ for free and total volatiles respectively). This was also observed by Anesi et al in Corvina berries (Anesi et al. 2015). This is well expected as the concentration and composition of aroma compounds change during berry development. Veraison is a brief physiological period marks the onset of berry ripening when an accumulation of sugars, anthocyanins, pigments, and additional phenols, but a smaller number of volatiles and precursors. Veraison is the most important incept of volatile change during berry development (Kalua and Boss 2009; Yuan and Qian 2016). After veraison, the berry chemistry changes due to high concentrations of glucose and fructose in the berries along with decreasing organic acid levels along with the synthesis of many precursors of aroma compounds and many aroma compounds (terpenes, norisoprenoids, esters, and thiols) (Coombe et al. 1987; CooMbe and McCarthy 2000; Ristic and Iland 2005; Kennedy 2002). Additionally, berry chemistry during both veraison and harvest is highly influenced by many factors including cultivar, rootstock, shading, climate, pest and disease pressures, and soil nutrients (Barnuud et al. 2014; Galletto et al. 2014; Main et al. 2002). This also explains why veraison in both years had lesser spread than harvest as 
indicated by PCA due to the presence of not many volatiles, but at harvest different microclimates and factors come into play to synthesize and differentiate many volatiles. Many volatile compounds and their precursors are formed after veraison.

The year is the second most important factor in explaining variation in both free and total volatiles in berries, explaining $42.53 \%$ and $20.91 \%$ of variation respectively. This was also observed in Corvina grapes by Anesi et al. (Anesi et al. 2015). The 2017 and 2018 seasons differed particularly in the amount and intensity of rainfall and temperature. Although total precipitation received in 2017 was higher than 2018, 2017 received only four significant precipitation greater than 0.5 inches whereas Arapitsas et al. 2020; Schueuermann et al. 2016):[abj]. The difference in rainfall has been found in our study and thus has been a major driver of change in berry volatiles in our study. Similarly, the water potential of the vines during harvest in 2017 than in 2018 was higher as soil moisture content was lower in 2017 compared to that of 2018 (data from chapter 5), suggesting the vines during harvest were (Ryona and Sacks 2013):0ij].

The use of rootstocks has a subtle but significant effect on free and total volatiles on Chambourcin berries (Fig 4.1 and Table S3, S4). This was also evident from the PCA scores plot where there was overlap between different root systems for both free and total volatiles (Fig 2). However, when used in a linear model as a factor of variation, rootstocks contributed 3-5\% of the variation in berry volatiles. Such small and subtle effect due to rootstocks had been previously observed in berry composition in Pinot noir (Berdeja et al. 2014), wine composition in Merlot, Syrah and Chardonnay (Harbertson and Keller 2011), aroma potential in Cabernet Sauvignon (Koundouras et al. 2009), berry and wine composition in shiraz (Olarte Mantilla et al. 2018). Most of these studies had 
attributed these differences due to rootstocks to change in canopy density, vine vigor, sun exposure, and shading. Changes in vigor, shading and solar exposure leads to microclimatic variations such as light intensity and temperature, that have been found to alter the synthesis and production of many berry volatiles and precursors (Kwasniewski et al. 2010; Asproudi et al. 2016). The few studies that have looked at the impact of rootstocks on volatiles, total aroma precursors were studied (Koundouras et al. 2009) or few selected compounds were studied (Romero et al. 2019). To identify this subtle effect of rootstocks on berry volatiles in under-explored cultivars such as Chambourcin, an untargeted approach thus provided the opportunity to identify compounds in an unbiased way.

Although irrigation was a treatment factor in our study, it had a subtle effect on both free and total volatiles, contributing only up to $4 \%$ of the variation, likely in part to the fairly wet seasons studied. Similar to rootstocks, irrigation treatments overlapped with each other, showing a subtle effect on volatiles as shown by the PCA score plot. Four PCs showed irrigation as a significant factor for free volatiles, whereas three PCs explained significant variation in total volatiles due to irrigation. RDI was significantly different from none and full irrigation in our study. Although prolonged and severe water stress has been found to have a negative effect on the growth, development, and yield of the plant, however, many studies have found the increase in the concentration of berry volatiles especially norisoprenoids due to limited water availability from deficit irrigation which is indirectly due to reduced vine vigor, increase in berry sun exposure and temperature and accelerated degradation of carotenoids (Qian et al. 2009; Bindon et al. 
2007). RDI might be a balance between severe water stress faced by plants in case of no irrigation and excess water availability that could result in excessive growth.

The free volatiles that we identified to be different between veraison and harvest are 2-Hexen-1-ol, (E)- Hexanal and 2-Hexenal, (E)-. All these compounds were present in higher concentrations in veraison than at harvest and have characteristic green herbaceous aromas. These volatiles were also found as major volatiles at veraison in Cabernet Sauvignon grapes (Kalua and Boss 2009). These C6 volatiles are mostly present as aldehydes during early development stages and transformed into alcohol during late development stages or harvest but are cultivar dependent (Kalua and Boss 2009). (E)-2hexenal and hexanal are derived from linolenic acid (C18:3) and linoleic acid (C18:2), at the same level as the lipoxygenase pathway (Kalua and Boss 2009). Both (E)-2-hexenal and hexanal showed a significant increase $(\mathrm{p}<0.05)$ around veraison followed by a decrease in the harvest sample in Cabernet Sauvignon due to an increase in alcohol dehydrogenase enzyme (Kalua and Boss 2009). It is found that the C6 alcohols continuously decreased during berry development whereas the C6 aldehyde began to decrease after reaching the harvest maturity (Fang and Qian 2012).

$\beta$-Damascenone, a C13 norisoprenoid is a major free volatile that is significantly impacted by rootstocks and irrigation. Chambourcin berries were found to have free $\beta$ Damascenone concentration up to $11.53 \mathrm{ug} / \mathrm{L}$ and total $\beta$-Damascenone up to $18.21 \mathrm{ug} / \mathrm{L}$. The previous study on hybrid grapes also found the concentration of free $\beta$-Damascenone up to $17.6 \mathrm{ug} / \mathrm{L}$ in St. Pepping grapes, $25 \mathrm{ug} / \mathrm{L}$ in Frontenac, $34.3 \mathrm{ug} / \mathrm{L}$ in St. Croix grapes, which is generally higher than that of vinifera cultivars (Slegers et al. 2015; Slegers et al. 2017). We found that the concentration of free $\beta$-Damascenone at veraison 
and harvest are similar to each other, with higher concentrations at veraison in 2017 and harvest in 2018. This is consistent with a previous study that found the concentration of free $\beta$-Damascenone to be consistent from veraison to harvest and reported accumulation around 5 days before veraison in Pinot noir grapes (Yuan and Qian 2016). $\beta$ -

Damascenone is an important C13 norisoprenoid important to red wine aroma and thus extensively studied, however, there are many conflicting results about its formation and development in grapes. Though $\beta$-Damascenone is known to be formed from carotenoid degradation mostly $\beta$-carotene and neoxanthin, its formation requires various rearrangements and catalysis which is still unknown.

Free $\beta$-Damascenone was found to be significantly impacted by rootstocks (Table 4.1) in both 2017 and 2018. Own-rooted Chambourcin berries were found to have the highest concentration of free $\beta$-Damascenone at veraison and harvest in both years (Figure 4.13A). This was in contrast to previous research on Shiraz wines, where (Z)Damascenone and (E)-Damascenone was lowest in concentration in own-rooted Shiraz, however, no berry volatiles was not included in that study (Olarte Mantilla et al. 2018). Similarly, irrigation also had a significant impact on free $\beta$-Damascenone in some years (Table 1). Berries from RDI treated vines had the highest concentration at veraison 2017 and harvest 2018. Previous studies have found that moderate water stress mediated through deficit irrigation increased the concentrations of $\beta$-Damascenone in berries and wines (Bindon et al. 2007; Qian et al. 2009). Water deficit could increase carotenoid cleavage dioxygenase (CCD) (Deluc et al. 2009) and thus have found to increase the concentration of C13 norisoprenoids (Bindon et al. 2007). 
$\beta$-Damascenone had been found to be in mostly glycosylated form in vinifera grapes and thus the bound $\beta$-Damascenone that are released from glycosides due to enzymatic hydrolysis was higher in concentration in comparison to the free fraction (Hampel et al. 2014; Qian et al. 2009; Pineau et al. 2007; Yuan and Qian 2016). Our results indicated a higher concentration of bound fraction in 2017 , however, in 2018, it is equal to or lower than a free fraction. To our knowledge, this is the first study that reported bound $\beta$ Damascenone in Chambourcin berries, however, our concentration level matches with those found in other interspecific hybrids (Slegers et al. 2015). The lower concentration in 2018 might be due to yearly variation in rainfall, temperature, and environmental factors that could impact the formation and storage of aroma molecules. It is not clear if this is the result of incomplete hydrolysis or due to inherent variation in Chambourcin berries. RDI was observed to be significantly different than other irrigation regimes in harvest 2017, which indicated that RDI is impacting vines in a different way than full irrigation or no irrigation.

\section{Conclusion:}

Using a metabolomics-based approach, we were able to identify subtle differences caused by rootstock and irrigation in the hybrid cultivar Chambourcin. Because of its inclusive nature, this approach provides advantages over the traditional targeted approach especially in understudied cultivar such as Chambourcin where there are limited studies regarding the volatile profile. We identified 279 and 259 metabolite features for free and total volatiles respectively and using multivariate approaches and linear modeling, we were able to identify the metabolites that significantly impact the variation caused due to factors in our study. While the major factor explaining most of the variation in berry 
volatiles is phenology and year (contributing up to $64 \%$ of the variation), rootstocks and irrigation contributed small (up to $8 \%$ of variation) but significant variation in free and total volatiles. The metabolites that explained variation due to phenology and year are mostly contributing green, herbaceous, and cut grass aromas, whereas $\beta$-Damascenone and methyl salicylate mostly explained variation due to rootstocks in free volatiles. Ethyl nonanoate and methyl salicylate explained major variation due to rootstocks for total volatiles. Irrigation is found to have a major impact on $\beta$-Damascenone, methyl salicylate, and 1-Dodecanol. We quantified $\beta$-Damascenone using authentic standard and found to be significantly higher in concentration in berries from own-rooted Chambourcin. $\beta$-Damascenone (RT 23.47), 2-Hexenal, (E )- (RT 8.9), Ethyl hexanoate (RT 9.19), and Phenylethyl alcohol (RT 25.38) are predictable in harvest from veraison. Compounds such as 1-Hexanol (RT 12.35), 2-Hexen-1-ol, (E )-, 2-Hexen-1-ol, (Z)-, 1Dodecanol, Ethyl 9-hexadecenoate, $\beta$-Damascenone, Phenylethyl alcohol, ethyl hexanoate are predictive between 2017 and 2018. Thus, using an untargeted approach, we were able to identify the significant metabolites that are impacted by various factors (irrigation, rootstock, phenology, and year) in Chambourcin. Future work related to this project will be to correlate wine and berry metabolites to see the predictability of wine metabolites based on berry metabolites. Since we collected multiple phenotype data in the same vines at the same time, correlations of berry and wine volatiles with other phenotypes ionomics, RNA seq, remote sensing, and physiology data to be able to explain what is happening in vines, that causes the observed changes in the berry metabolites. 


\section{References:}

Alessandrini M, Battista F, Panighel A, Flamini R, Tomasi D (2017) Effect of pre-bloom leaf removal on grape aroma composition and wine sensory profile of Semillon cultivar. J Sci Food Agric. doi:10.1002/jsfa.8638

Ali MB, Howard S, Chen S, Wang Y, Yu O, Kovacs LG, Qiu W (2011) Berry skin development in Norton grape: distinct patterns of transcriptional regulation and flavonoid biosynthesis. BMC plant biology 11 (1):1

Ambers CP (2013) A historical hypothesis on the origin of the norton grape. Journal of Wine Research 24 (2):85-95. doi:10.1080/09571264.2012.747087

Ambers RK, Ambers CP (2004) Dr. Daniel Norborne Norton and the origin of the Norton grape. Am Wine Soc J 36 (3):77-87

Anesi A, Stocchero M, Dal Santo S, Commisso M, Zenoni S, Ceoldo S, Tornielli GB, Siebert TE, Herderich M, Pezzotti M (2015) Towards a scientific interpretation of the terroir concept: plasticity of the grape berry metabolome. BMC plant biology 15 (1):191

Arapitsas P, Ugliano M, Marangon M, Piombino P, Rolle L, Gerbi V, Versari A, Mattivi F (2020) Use of Untargeted Liquid Chromatography-Mass Spectrometry Metabolome To Discriminate Italian Monovarietal Red Wines, Produced in Their Different Terroirs. Journal of Agricultural and Food Chemistry. doi:10.1021/acs.jafc.0c00879

Asproudi A, Petrozziello M, Cavalletto S, Guidoni S (2016) Grape aroma precursors in cv. Nebbiolo as affected by vine microclimate. Food chemistry 211:947-956

Balint G, Reynolds AG (2017) Irrigation level and time of imposition impact vine physiology, yield components, fruit composition and wine quality of Ontario Chardonnay. Scientia horticulturae 214:252-272

Barnuud NN, Zerihun A, Gibberd M, Bates B (2014) Berry composition and climate: responses and empirical models. International journal of biometeorology 58 (6):1207-1223

Battilana J, Costantini L, Emanuelli F, Sevini F, Segala C, Moser S, Velasco R, Versini G, Grando MS (2009) The 1-deoxy-d-xylulose 5-phosphate synthase gene colocalizes with a major QTL affecting monoterpene content in grapevine. Theoretical and Applied Genetics 118 (4):653-669

Berdeja M, Hilbert G, Dai ZW, Lafontaine M, Stoll M, Schultz HR, Delrot S (2014) Effect of water stress and rootstock genotype on Pinot Noir berry composition. Australian Journal of Grape and Wine Research 20 (3):409-421. doi:10.1111/ajgw.12091

Berdeja M, Nicolas P, Kappel C, Dai ZW, Hilbert G, Peccoux A, Lafontaine M, Ollat N, Gomès E, Delrot S (2015) Water limitation and rootstock genotype interact to alter grape berry metabolism through transcriptome reprogramming. Horticulture research 2:15012

Bianco RL, Farina V, Avellone G, Filizzola F, Agozzino P (2008) Fruit quality and volatile fraction of 'Pink Lady'apple trees in response to rootstock vigor and partial rootzone drying. Journal of the Science of Food and Agriculture 88 (8):1325-1334 
Biasoto ACT, Netto FM, Marques EJN, da Silva MAAP (2014) Acceptability and preference drivers of red wines produced from Vitis labrusca and hybrid grapes. Food Research International 62:456-466. doi:10.1016/j.foodres.2014.03.052

Bindon KA, Dry PR, Loveys BR (2007) Influence of Plant Water Status on the Production of C13-Norisoprenoid Precursors in Vitis vinifera L. Cv. Cabernet Sauvignon Grape Berries. Journal of Agricultural and Food Chemistry 55 (11):4493-4500. doi:10.1021/jf063331p

Bisson LF, Waterhouse AL, Ebeler SE, Walker MA, Lapsley JT (2002a) The present and future of the international wine industry. Nature 418 (6898):696

Bisson LF, Waterhouse AL, Ebeler SE, Walker MA, Lapsley JT (2002b) The present and future of the international wine industry. Nature 418 (6898):696-699. doi:10.1038/nature01018

Black C, Parker M, Siebert T, Capone D, Francis I (2015) Terpenoids and their role in wine flavour: recent advances. Australian journal of grape and wine research 21:582-600

Callejon RM, Margulies B, Hirson GD, Ebeler SE (2012) Dynamic Changes in Volatile Compounds during Fermentation of Cabernet Sauvignon Grapes with and without Skins. American Journal of Enology and Viticulture 63 (3):301-312. doi:10.5344/ajev.2012.12009

Canuti V, Conversano M, Calzi ML, Heymann H, Matthews MA, Ebeler SE (2009) Headspace solid-phase microextraction-gas chromatography-mass spectrometry for profiling free volatile compounds in Cabernet Sauvignon grapes and wines. J Chromatogr A 1216 (15):3012-3022. doi:10.1016/j.chroma.2009.01.104

Carlin S, Masuero D, Guella G, Vrhovsek U, Mattivi F (2019) Methyl Salicylate Glycosides in Some Italian Varietal Wines. Molecules 24 (18). doi:10.3390/molecules24183260

Carrasco-Quiroz M, Martínez-Gil AM, Gutiérrez-Gamboa G, Moreno-Simunovic Y (2020) Effect of rootstocks on volatile composition of Merlot wines. Journal of the Science of Food and Agriculture 100 (8):3517-3524. doi:10.1002/jsfa.10395

Chapman DM, Roby G, Ebeler SE, Guinard JX, Matthews MA (2005) Sensory attributes of Cabernet Sauvignon wines made from vines with different water status. Australian Journal of Grape and Wine Research 11 (3):339-347

Chaves MM, Santos TP, Souza Cd, Ortuño M, Rodrigues M, Lopes C, Maroco J, Pereira JS (2007) Deficit irrigation in grapevine improves water-use efficiency while controlling vigour and production quality. Annals of Applied Biology 150 (2):237-252

Chisholm MG, Guiher LA, Vonah TM, Beaumont JL (1994) Comparison of some French-American hybrid wines with White Riesling using gas chromatographyolfactometry. American journal of enology and viticulture 45 (2):201-212

Commisso M, Strazzer P, Toffali K, Stocchero M, Guzzo F (2013) Untargeted metabolomics: an emerging approach to determine the composition of herbal products. Computational and structural biotechnology journal 4 (5):e201301007

Cookson SJ, Ollat N (2013) Grafting with rootstocks induces extensive transcriptional reprogramming in the shoot apical meristem of grapevine. BMC Plant Biology 13 (1):147. doi:10.1186/1471-2229-13-147 
Coombe B, BOVIO M, SCHNEIDER A (1987) Solute accumulation by grape pericarp cells: v. relationship to berry size and the effects of defoliation. Journal of Experimental Botany 38 (11):1789-1798

CooMbe BG, McCarthy M (2000) Dynamics of grape berry growth and physiology of ripening. Australian journal of grape and wine research 6 (2):131-135

Cousins P (2005) Evolution, genetics, and breeding: viticultural applications of the origins of our rootstocks. Grapevine Rootstocks: Current Use, Research, and Application: 1

Cuadros-Inostroza A, Giavalisco P, Hummel J, Eckardt A, Willmitzer L, Peña-Cortés H (2010) Discrimination of wine attributes by metabolome analysis. Analytical Chemistry 82 (9):3573-3580

Cuadros-Inostroza A, Ruíz-Lara S, González E, Eckardt A, Willmitzer L, Peña-Cortés H (2016) GC-MS metabolic profiling of Cabernet Sauvignon and Merlot cultivars during grapevine berry development and network analysis reveals a stage-and cultivar-dependent connectivity of primary metabolites. Metabolomics 12 (2):39

de Pinho PG, Bertrand A (1995) Analytical determination of furaneol (2, 5-dimethyl-4hydroxy-3 (2H)-furanone). Application to differentiation of white wines from hybrid and various Vitis vinifera cultivars. American journal of enology and viticulture 46 (2):181-186

De Vos RC, Moco S, Lommen A, Keurentjes JJ, Bino RJ, Hall RD (2007) Untargeted large-scale plant metabolomics using liquid chromatography coupled to mass spectrometry. Nature protocols 2 (4):778

Deluc LG, Quilici DR, Decendit A, Grimplet J, Wheatley MD, Schlauch KA, Mérillon JM, Cushman JC, Cramer GR (2009) Water deficit alters differentially metabolic pathways affecting important flavor and quality traits in grape berries of Cabernet Sauvignon and Chardonnay. BMC genomics 10 (1):212

Dempsey DMA, Vlot AC, Wildermuth MC, Klessig DF (2011) Salicylic Acid biosynthesis and metabolism. The arabidopsis book 9:e0156-e0156. doi:10.1199/tab.0156

des Gachons CP, Leeuwen CV, Tominaga T, Soyer JP, Gaudillère JP, Dubourdieu D (2005) Influence of water and nitrogen deficit on fruit ripening and aroma potential of Vitis vinifera $\mathrm{L} \mathrm{cv}$ Sauvignon blanc in field conditions. Journal of the Science of Food and Agriculture 85 (1):73-85

Doligez A, Audiot E, Baumes R, This P (2006) QTLs for muscat flavor and monoterpenic odorant content in grapevine (Vitis vinifera L.). Molecular Breeding 18 (2):109-125

Dunemann F, Ulrich D, Boudichevskaia A, Grafe C, Weber W (2009) QTL mapping of aroma compounds analysed by headspace solid-phase microextraction gas chromatography in the apple progeny 'Discovery' $\times$ 'Prima'. Molecular breeding 23 (3):501-521

Ebeler SE (2001) Analytical Chemistry: Unlocking the Secrets of Wine Flavor. Food Reviews International 17 (1):45-64. doi:10.1081/fri-100000517

Ebeler SE, Thorngate JH (2009) Wine chemistry and flavor: looking into the crystal glass. J Agric Food Chem 57 (18):8098-8108. doi:10.1021/jf9000555

El-Ansary DO, Okamoto G (2007) Vine Water Relations and Quality of \&lsquo;Muscat of Alexandria\&rsquo; Table Grapes Subjected to Partial Root-zone Drying and 
Regulated Deficit Irrigation. Journal of the Japanese Society for Horticultural Science 76 (1):13-19. doi:10.2503/jjshs.76.13

Escudero A, Campo E, Fariña L, Cacho J, Ferreira V (2007) Analytical characterization of the aroma of five premium red wines. Insights into the role of odor families and the concept of fruitiness of wines. Journal of Agricultural and Food Chemistry 55 (11):4501-4510

Ezzahouani A, Williams LE (1995) The influence of rootstock on leaf water potential, yield, and berry composition of Ruby Seedless grapevines. American Journal of Enology and Viticulture 46 (4):559-563

Fang Y, Qian MC (2006) Quantification of selected aroma-active compounds in Pinot noir wines from different grape maturities. Journal of agricultural and food chemistry 54 (22):8567-8573

Fang Y, Qian MC (2012) Development of C6 and Other Volatile Compounds in Pinot Noir Grapes Determined by Stir Bar Sorptive Extraction-GC-MS. In: Flavor Chemistry of Wine and Other Alcoholic Beverages, vol 1104. ACS Symposium Series, vol 1104. American Chemical Society, pp 81-99. doi:doi:10.1021/bk2012-1104.ch006

10.1021/bk-2012-1104.ch006

Ferreira V, Lopez R (2019) The Actual and Potential Aroma of Winemaking Grapes. Biomolecules 9 (12):818

Ferreira V, López R, Cacho JF (2000) Quantitative determination of the odorants of young red wines from different grape varieties. Journal of the Science of Food and Agriculture 80 (11):1659-1667

Ferris H, Zheng L, Walker M (2012) Resistance of grape rootstocks to plant-parasitic nematodes. Journal of nematology 44 (4):377

Flamini R, De Rosso M, Panighel A, Dalla Vedova A, De Marchi F, Bavaresco L (2014) Profiling of grape monoterpene glycosides (aroma precursors) by ultra-high performance-liquid chromatography-high resolution mass spectrometry (UHPLC/QTOF). J Mass Spectrom 49 (12):1214-1222. doi:10.1002/jms.3441

Francis I, Newton J (2005) Determining wine aroma from compositional data. Australian Journal of Grape and Wine Research 11 (2):114-126

Fung RW, Gonzalo M, Fekete C, Kovacs LG, He Y, Marsh E, McIntyre LM, Schachtman DP, Qiu W (2008) Powdery mildew induces defense-oriented reprogramming of the transcriptome in a susceptible but not in a resistant grapevine. Plant physiology 146 (1):236-249

Galletto L, Barisan L, Boatto V, AC Costantini E, Lorenzetti R, Pomarici E, Vecchio R (2014) More crop for drop-climate change and wine: an economic evaluation of a new drought-resistant rootstock. Recent patents on food, nutrition \& agriculture 6 (2):100-112

Ghaste M, Narduzzi L, Carlin S, Vrhovsek U, Shulaev V, Mattivi F (2015) Chemical composition of volatile aroma metabolites and their glycosylated precursors that can uniquely differentiate individual grape cultivars. Food Chem 188:309-319. doi:10.1016/j.foodchem.2015.04.056

Gika HG, Theodoridis GA, Plumb RS, Wilson ID (2014) Current practice of liquid chromatography-mass spectrometry in metabolomics and metabonomics. J Pharm Biomed Anal 87:12-25. doi:10.1016/j.jpba.2013.06.032 
Gouthu S, Deluc LG (2015) Timing of ripening initiation in grape berries and its relationship to seed content and pericarp auxin levels. BMC plant biology 15 (1):46

Gowda H, Ivanisevic J, Johnson CH, Kurczy ME, Benton HP, Rinehart D, Nguyen T, Ray J, Kuehl J, Arevalo B, Westenskow PD, Wang J, Arkin AP, Deutschbauer AM, Patti GJ, Siuzdak G (2014) Interactive XCMS Online: Simplifying Advanced Metabolomic Data Processing and Subsequent Statistical Analyses. Analytical Chemistry 86 (14):6931-6939. doi:10.1021/ac500734c

Gross JH (2006) Mass spectrometry: a textbook. Springer Science \& Business Media,

Gu S, Read PE, Gamet S (2005) Performance of 'Gewurztraminer' on six rootstocks under marginal climatic conditions. Grapevine Rootstocks: Current Use, Research, and Application:57

Guillaumie S, Ilg A, Réty S, Brette M, Trossat-Magnin C, Decroocq S, Léon C, Keime C, Ye T, Baltenweck-Guyot R (2013) Genetic analysis of the biosynthesis of 2methoxy-3-isobutylpyrazine, a major grape-derived aroma compound impacting wine quality. Plant physiology 162 (2):604-615

Gunata Z, Vallier M, Sapis J, Baumes R, Bayonove C (1994) Enzymatic synthesis of monoterpenyl $\beta$-D-glucosides by various $\beta$-glucosidases. Enzyme and microbial technology 16 (12):1055-1058

Guth H (1997) Quantitation and Sensory Studies of Character Impact Odorants of Different White Wine Varieties. Journal of Agricultural and Food Chemistry 45 (8):3027-3032. doi:10.1021/jf970280a

Hampel D, Robinson AL, Johnson A, Ebeler SE (2014) Direct hydrolysis and analysis of glycosidically bound aroma compounds in grapes and wines: comparison of hydrolysis conditions and sample preparation methods. Australian journal of grape and wine research 20 (3):361-377

Harbertson JF, Keller M (2011) Rootstock Effects on Deficit-Irrigated Winegrapes in a Dry Climate: Grape and Wine Composition. American Journal of Enology and Viticulture 63 (1):40-48. doi:10.5344/ajev.2011.11079

Hernandez-Orte P, Concejero B, Astrain J, Lacau B, Cacho J, Ferreira V (2015) Influence of viticulture practices on grape aroma precursors and their relation with wine aroma. Journal of the Science of Food and Agriculture 95 (4):688-701

Hjelmeland AK, Ebeler SE (2014) Glycosidically Bound Volatile Aroma Compounds in Grapes and Wine: A Review. American Journal of Enology and Viticulture 66 (1):1-11. doi:10.5344/ajev.2014.14104

Hjelmeland AK, Zweigenbaum J, Ebeler SE (2015) Profiling monoterpenol glycoconjugation in Vitis vinifera L. cv. Muscat of Alexandria using a novel putative compound database approach, high resolution mass spectrometry and collision induced dissociation fragmentation analysis. Anal Chim Acta 887:138147. doi:10.1016/j.aca.2015.06.026

Hogan S, Zhang L, Li J, Zoecklein B, Zhou K (2009) Antioxidant properties and bioactive components of Norton (Vitis aestivalis) and Cabernet Franc (Vitis vinifera) wine grapes. LWT-Food Science and Technology 42 (7):1269-1274

Holopainen JK, Blande JD (2012) Molecular plant volatile communication. In: Sensing in nature. Springer, pp 17-31 
Ilc T, Werck-Reichhart D, Navrot N (2016) Meta-analysis of the core aroma components of grape and wine aroma. Frontiers in Plant Science 7:1472

Jackson RS (2008) Wine science: principles and applications. Academic press,

James G, Witten D, Hastie T, Tibshirani R (2013) An introduction to statistical learning, vol 112. Springer,

Jogaiah S, Striegler KR, Bergmeier E, Harris J (2012) Influence of cluster exposure to Sun on fruit composition of 'Norton'grapes (vitis estivalis michx) in Missouri. International journal of fruit science 12 (4):410-426

Jogaiah S, Striegler KR, Bergmeier E, Harris J (2013) Influence of canopy management practices on canopy characteristics, yield, and fruit composition of 'Norton'grapes (Vitis aestivalis Michx). International journal of fruit science 13 (4):441-458

Jones T, Cullis BR, Clingeleffer PR, Rühl E (2009a) Effects of novel hybrid and traditional rootstocks on vigour and yield components of Shiraz grapevines. Australian Journal of Grape and Wine Research 15 (3):284-292

JONES TH, CULLIS BR, CLINGELEFFER PR, RÜHL EH (2009b) Effects of novel hybrid and traditional rootstocks on vigour and yield components of Shiraz grapevines. Australian Journal of Grape and Wine Research 15 (3):284-292. doi:10.1111/j.1755-0238.2009.00061.x

Kalua C, Boss PK (2010) Comparison of major volatile compounds from Riesling and Cabernet Sauvignon grapes (Vitis vinifera L.) from fruitset to harvest. Australian Journal of Grape and Wine Research 16 (2):337-348

Kalua CM, Boss PK (2009) Evolution of volatile compounds during the development of Cabernet Sauvignon grapes (Vitis vinifera L.). Journal of agricultural and food chemistry 57 (9):3818-3830

Katajamaa M, Miettinen J, Orešič M (2006) MZmine: toolbox for processing and visualization of mass spectrometry based molecular profile data. Bioinformatics 22 (5):634-636

Keller M, Mills LJ, Harbertson JF (2012) Rootstock effects on deficit-irrigated winegrapes in a dry climate: Vigor, yield formation, and fruit ripening. American Journal of Enology and Viticulture 63 (1):29-39

Kennedy J (2002) Understanding grape berry development. Practical winery \& vineyard $4: 1-5$

Kliewer W (1965) Changes in Concentration of Glucose, Fructose, and Total Soluble Solids in Flowers and Berries of Vitis Vlnifera. American Journal of Enology and Viticulture 16 (2):101-110

Kliewer WM, Howarth L, Omori M (1967) Concentrations of tartaric acid and malic acids and their salts in Vitis vinifera grapes. American Journal of Enology and Viticulture 18 (1):42-54

Kolor MG (1983) Identification of an important new flavor compound in Concord grape: ethyl-3-mercaptopropionate. Journal of Agricultural and Food Chemistry 31 (5):1125-1127

Kotseridis Y, Baumes RL, Skouroumounis GK (1999) Quantitative determination of free and hydrolytically liberated $\beta$-damascenone in red grapes and wines using a stable isotope dilution assay. Journal of Chromatography A 849 (1):245-254. doi:https://doi.org/10.1016/S0021-9673(99)00540-3 
Koundouras S, Hatzidimitriou E, Karamolegkou M, Dimopoulou E, Kallithraka S, Tsialtas JT, Zioziou E, Nikolaou N, Kotseridis Y (2009) Irrigation and rootstock effects on the phenolic concentration and aroma potential of Vitis vinifera L. cv. cabernet sauvignon grapes. J Agric Food Chem 57 (17):7805-7813. doi:10.1021/jf901063a

Koundouras S, Tsialtas IT, Zioziou E, Nikolaou N (2008) Rootstock effects on the adaptive strategies of grapevine (Vitis vinifera L. cv. Cabernet-Sauvignon) under contrasting water status: Leaf physiological and structural responses. Agriculture, Ecosystems \& Environment 128 (1):86-96. doi:https://doi.org/10.1016/j.agee.2008.05.006

Kwasniewski MT, Vanden Heuvel JE, Pan BS, Sacks GL (2010) Timing of cluster light environment manipulation during grape development affects $\mathrm{C} 13$ norisoprenoid and carotenoid concentrations in Riesling. Journal of agricultural and food chemistry 58 (11):6841-6849

Lee J-E, Hwang G-S, Van Den Berg F, Lee C-H, Hong Y-S (2009) Evidence of vintage effects on grape wines using 1H NMR-based metabolomic study. Analytica chimica acta 648 (1):71-76

Liland KH (2011) Multivariate methods in metabolomics - from pre-processing to dimension reduction and statistical analysis. TrAC Trends in Analytical Chemistry 30 (6):827-841. doi:https://doi.org/10.1016/j.trac.2011.02.007

Liu D, Xing R-R, Li Z, Yang D-M, Pan Q-H (2016) Evolution of volatile compounds, aroma attributes, and sensory perception in bottle-aged red wines and their correlation. European Food Research and Technology 242 (11):1937-1948. doi:10.1007/s00217-016-2693-1

Liu HF, Wu BH, Fan PG, Li SH, Li LS (2006) Sugar and acid concentrations in 98 grape cultivars analyzed by principal component analysis. Journal of the Science of Food and Agriculture 86 (10):1526-1536

Maimaitiyiming M, Ghulam A, Bozzolo A, Wilkins JL, Kwasniewski MT (2017) Early detection of plant physiological responses to different levels of water stress using reflectance spectroscopy. Remote Sensing 9 (7):745

Main G (2005) Growing and vinting Cynthiana. Norton grapes. Proceedings of the 24th Annual Horticulture Industries Show ...,

Main G, Morris J, Striegler K (2002) Rootstock effects on Chardonel productivity, fruit, and wine composition. American Journal of Enology and Viticulture 53 (1):37-40

Main GL, Morris JR (2008) Impact of pruning methods on yield components and juice and wine composition of Cynthiana grapes. American journal of enology and viticulture 59 (2):179-187

Mansfield AK, Schirle-Keller J-P, Reineccius GA (2011) Identification of odor-impact compounds in red table wines produced from Frontenac grapes. American journal of enology and viticulture:ajev. 2011.10067

Mateo J, Jiménez M (2000) Monoterpenes in grape juice and wines. Journal of chromatography A 881 (1-2):557-567

Mato I, Suárez-Luque S, Huidobro JF (2005) A review of the analytical methods to determine organic acids in grape juices and wines. Food Research International 38 (10):1175-1188 
Mendes-Pinto MM (2009) Carotenoid breakdown products the-norisoprenoids-in wine aroma. Archives of Biochemistry and Biophysics 483 (2):236-245. doi:https://doi.org/10.1016/j.abb.2009.01.008

Migicovsky Z, Harris ZN, Klein LL, Li M, McDermaid A, Chitwood DH, Fennell A, Kovacs LG, Kwasniewski M, Londo JP, Ma Q, Miller AJ (2019) Rootstock effects on scion phenotypes in a 'Chambourcin' experimental vineyard. Horticulture Research 6 (1):64. doi:10.1038/s41438-019-0146-2

Mirás-Avalos JM, Intrigliolo DS (2017) Grape Composition under Abiotic Constrains: Water Stress and Salinity. Frontiers in Plant Science 8 (851). doi:10.3389/fpls.2017.00851

Moyer J, Mattick L (1976) Determination of methyl anthranilate in wines. American Journal of Enology and Viticulture 27 (3):134-135

Mudge K, Janick J, Scofield S, Goldschmidt EE (2009) 9 A History of Grafting. Horticultural reviews 35:437

Narduzzi L, Stanstrup J, Mattivi F (2015) Comparing Wild American Grapes with Vitis vinifera: A Metabolomics Study of Grape Composition. J Agric Food Chem 63 (30):6823-6834. doi:10.1021/acs.jafc.5b01999

Nelson CC, Kennedy JA, Zhang Y, Kurtural SK (2015) Applied Water and Rootstock Affect Productivity and Anthocyanin Composition of Zinfandel in Central California. American Journal of Enology and Viticulture 67 (1):18-28. doi:10.5344/ajev.2015.15043

Nelson R, Acree T, Lee C, Butts R (1977) Methyl anthranilate as an aroma constituent of American wine. Journal of Food Science 42 (1):57-59

Nisbet MA, Martinson TE, Mansfield AK (2014) Accumulation and Prediction of Yeast Assimilable Nitrogen in New York Winegrape Cultivars. American Journal of Enology and Viticulture 65 (3):325-332. doi:10.5344/ajev.2014.13130

Noble A, Cole V (2003) Flavor chemistry. In: Fermented Beverage Production. Springer, pp 393-412

Noble AC, Ebeler SE (2002) Use of multivariate statistics in understanding wine flavor. Food Reviews International 18 (1):1-20. doi:10.1081/fri-120003414

Olarte Mantilla SM, Collins C, Iland PG, Kidman CM, Ristic R, Boss PK, Jordans C, Bastian SEP (2018) Shiraz (<em>Vitis vinifera</em> L.) Berry and Wine Sensory Profiles and Composition Are Modulated by Rootstocks. American Journal of Enology and Viticulture 69 (1):32-44. doi:10.5344/ajev.2017.17017

Ollat N, Bordenave L, Tandonnet J-P, Boursiquot J-M, Marguerit E Grapevine rootstocks: origins and perspectives. In: I International Symposium on Grapevine Roots 1136, 2014. pp 11-22

Otto M (2016) Chemometrics: statistics and computer application in analytical chemistry. John Wiley \& Sons,

Ough C, Cook J, Lider L (1968) Rootstock-scion interactions concerning wine making. II. Wine compositional and sensory changes attributed to rootstock and fertilizer level differences. American Journal of Enology and Viticulture 19 (4):254-265

Parker M, Capone DL, Francis IL, Herderich MJ (2017) Aroma Precursors in Grapes and Wine: Flavor Release during Wine Production and Consumption. J Agric Food Chem. doi:10.1021/acs.jafc.6b05255 
Pineau B, Barbe J-C, Van Leeuwen C, Dubourdieu D (2007) Which Impact for $\beta$ Damascenone on Red Wines Aroma? Journal of Agricultural and Food Chemistry 55 (10):4103-4108. doi:10.1021/jf070120r

Plata C, Millan C, Mauricio J, Ortega J (2003) Formation of ethyl acetate and isoamyl acetate by various species of wine yeasts. Food Microbiology 20 (2):217-224

Polaskova P, Herszage J, Ebeler SE (2008) Wine flavor: chemistry in a glass. Chem Soc Rev 37 (11):2478-2489. doi:10.1039/b714455p

Poole C (2010) Chromatography: Concepts and Contrasts. Springer,

Qian MC, Fang Y, Shellie K (2009) Volatile Composition of Merlot Wine from Different Vine Water Status. Journal of Agricultural and Food Chemistry 57 (16):74597463. doi:10.1021/jf9009558

Rambla JL, Trapero-Mozos A, Diretto G, Rubio-Moraga A, Granell A, Gomez-Gomez L, Ahrazem O (2016) Gene-Metabolite Networks of Volatile Metabolism in Airen and Tempranillo Grape Cultivars Revealed a Distinct Mechanism of Aroma Bouquet Production. Front Plant Sci 7:1619. doi:10.3389/fpls.2016.01619

Rapp A, Mandery H (1986) Wine aroma. Experientia 42 (8):873-884

Reisch BI, Goodman RN, Martens M-H, Weeden NF (1993) The Relationship Between Norton and Cynthiana, Red. Wine Cultivars Derived from Vitis aestivalis. American journal of enology and viticulture 44 (4):441-444

Reisch BI, Owens CL, Cousins PS (2012) Grape. In: Fruit breeding. Springer, pp 225262

Reynolds AG, Fuleki T, Evans W (1982) Inheritance of methyl anthranilate and total volatile esters in Vitis spp. American Journal of Enology and Viticulture 33 (1):14-19

Reynolds AG, Lowrey WD, Tomek L, Hakimi J, De Savigny C (2007) Influence of irrigation on vine performance, fruit composition, and wine quality of Chardonnay in a cool, humid climate. American Journal of Enology and Viticulture 58 (2):217-228

Reynolds AG, Wardle DA (2001) Rootstocks impact vine performance and fruit composition of grapes in British Columbia. HortTechnology 11 (3):419-427

Rice S, Tursumbayeva M, Clark M, Greenlee D, Dharmadhikari M, Fennell A, Koziel JA (2019) Effects of harvest time on the aroma of white wines made from cold-hardy Brianna and Frontenac gris grapes using headspace solid-phase microextraction and gas chromatography-mass spectrometry-olfactometry. Foods 8 (1):29

Ristic R, Iland PG (2005) Relationships between seed and berry development of Vitis vinifera L. cv Shiraz: developmental changes in seed morphology and phenolic composition. Australian Journal of grape and wine research 11 (1):43-58

Robinson AL, Boss PK, Solomon PS, Trengove RD, Heymann H, Ebeler SE (2014) Origins of grape and wine aroma. Part 1. Chemical components and viticultural impacts. American Journal of Enology and Viticulture 65 (1):1-24

Robinson AL, Ebeler SE, Heymann H, Boss PK, Solomon PS, Trengove RD (2009) Interactions between wine volatile compounds and grape and wine matrix components influence aroma compound headspace partitioning. J Agric Food Chem 57 (21):10313-10322. doi:10.1021/jf902586n

Romero P, Botía P, del Amor FM, Gil-Muñoz R, Flores P, Navarro JM (2019) Interactive effects of the rootstock and the deficit irrigation technique on wine composition, 
nutraceutical potential, aromatic profile, and sensory attributes under semiarid and water limiting conditions. Agricultural Water Management 225:105733. doi:https://doi.org/10.1016/j.agwat.2019.105733

Roullier-Gall C, Witting M, Gougeon RD, Schmitt-Kopplin P (2014a) High precision mass measurements for wine metabolomics. Frontiers in chemistry 2:102

Roullier-Gall C, Witting M, Gougeon RD, Schmitt-Kopplin P (2014b) High precision mass measurements for wine metabolomics. Frontiers in Chemistry 2 (102). doi:10.3389/fchem.2014.00102

Ruffner H (1982a) Metabolism of tartaric and malic acids in Vitis V a review. A Vitis-,: $247 \mathrm{~V} 259$

Ruffner H (1982b) Metabolism of tartaric and malic acids in Vitis: A review-Part B. Vitis $21(247-259): 65$

Ryona I, Sacks GL (2013) Behavior of glycosylated monoterpenes, C13-norisoprenoids, and benzenoids in Vitis vinifera cv. Riesling during ripening and following hedging. In: Carotenoid cleavage products. ACS Publications, pp 109-124

Sabbatini P, Howell GS (2013) Rootstock scion interaction and effects on vine vigor, phenology, and cold hardiness of interspecific hybrid grape cultivars (Vitis spp.). International journal of fruit science 13 (4):466-477

Sacks GL, Gates MJ, Ferry FX, Lavin EH, Kurtz AJ, Acree TE (2012) Sensory threshold of 1,1,6-trimethyl-1,2-dihydronaphthalene (TDN) and concentrations in young Riesling and non-Riesling wines. J Agric Food Chem 60 (12):2998-3004. doi:10.1021/jf205203b

Saito K, Matsuda F (2010) Metabolomics for functional genomics, systems biology, and biotechnology. Annu Rev Plant Biol 61:463-489. doi:10.1146/annurev.arplant.043008.092035

Sapkota S, Chen L-L, Schreiner K, Ge H, Hwang C-F (2015) A phenotypic study of Botrytis bunch rot resistance in Vitis aestivalis-derived 'Norton'grape. Tropical Plant Pathology 40 (4):279-282

Sapkota S, Chen L-L, Yang S, Hyma KE, Cadle-Davidson L, Hwang C-F (2019) Construction of a high-density linkage map and QTL detection of downy mildew resistance in Vitis aestivalis-derived 'Norton'. Theoretical and applied genetics 132 (1):137-147

Savoi S, Wong DCJ, Arapitsas P, Miculan M, Bucchetti B, Peterlunger E, Fait A, Mattivi F, Castellarin SD (2016) Transcriptome and metabolite profiling reveals that prolonged drought modulates the phenylpropanoid and terpenoid pathway in white grapes (Vitis vinifera L.). BMC Plant Biology 16 (1):67. doi:10.1186/s12870-016-0760-1

Schrimpe-Rutledge AC, Codreanu SG, Sherrod SD, McLean JA (2016) Untargeted metabolomics strategies - challenges and emerging directions. Journal of the American Society for Mass Spectrometry 27 (12):1897-1905

Schueuermann C, Khakimov B, Engelsen SB, Bremer P, Silcock P (2016) GC-MS Metabolite Profiling of Extreme Southern Pinot noir Wines: Effects of Vintage, Barrel Maturation, and Fermentation Dominate over Vineyard Site and Clone Selection. Journal of Agricultural and Food Chemistry 64 (11):2342-2351. doi:10.1021/acs.jafc.5b05861 
Schueuermann C, Steel CC, Blackman JW, Clark AC, Schwarz LJ, Moraga J, Collado IG, Schmidtke LM (2019) A GC-MS untargeted metabolomics approach for the classification of chemical differences in grape juices based on fungal pathogen. Food chemistry 270:375-384

Schwab W, Wüst M (2015) Understanding the Constitutive and Induced Biosynthesis of Mono- and Sesquiterpenes in Grapes (Vitis vinifera): A Key to Unlocking the Biochemical Secrets of Unique Grape Aroma Profiles. Journal of Agricultural and Food Chemistry 63 (49):10591-10603. doi:10.1021/acs.jafc.5b04398

Sefton MA, Skouroumounis GK, Elsey GM, Taylor DK (2011) Occurrence, Sensory Impact, Formation, and Fate of Damascenone in Grapes, Wines, and Other Foods and Beverages. Journal of Agricultural and Food Chemistry 59 (18):9717-9746. doi:10.1021/jf201450q

Serra I, Strever A, Myburgh P, Deloire A (2014) the interaction between rootstocks and cultivars ( $\mathrm{V}$ itis vinifera L.) to enhance drought tolerance in grapevine. Australian Journal of Grape and Wine Research 20 (1):1-14

Shure KB, Acree TE (1994) Changes in the odor-active compounds in Vitis labruscana $\mathrm{cv}$. Concord during growth and development. Journal of agricultural and food chemistry $42(2): 350-353$

Skinkis PA, Bordelon BP, Wood KV (2008) Comparison of monoterpene constituents in Traminette, Gewürztraminer, and Riesling winegrapes. American journal of enology and viticulture 59 (4):440-445

Slegers A, Angers P, Ouellet E, Truchon T, Pedneault K (2015) Volatile Compounds from Grape Skin, Juice and Wine from Five Interspecific Hybrid Grape Cultivars Grown in Quebec (Canada) for Wine Production. Molecules 20 (6):10980-11016. doi:10.3390/molecules200610980

Slegers A, Angers P, Pedneault K (2017) Volatile Compounds from Must and Wines from Five White Grape Varieties. Journal of Food Chemistry and Nanotechnology 03 (01). doi:10.17756/jfcn.2017-031

Stockert CM, Bisson LF, Adams DO, Smart DR (2013) Nitrogen status and fermentation dynamics for Merlot on two rootstocks. American Journal of Enology and Viticulture 64 (2):195-202

Striegler R, Howell G (2015) The influence of rootstock on the cold-hardiness of Seyval grapevines 1 . Primary and secondary effects on growth, canopy development, yield, fruit quality and cold hardiness. VITIS-Journal of Grapevine Research 30 (1): 1

Striegler R, Howell G, Flore J (1993) Influence of rootstock on the response of Seyval grapevines to flooding stress. American journal of enology and viticulture 44 (3):313-319

Šuklje K, Carlin S, Stanstrup J, Antalick G, Blackman JW, Meeks C, Deloire A, Schmidtke LM, Vrhovsek U (2019) Unravelling wine volatile evolution during Shiraz grape ripening by untargeted HS-SPME-GC $\times$ GC-TOFMS. Food chemistry 277:753-765

Sun Q, Gates MJ, Lavin EH, Acree TE, Sacks GL (2011a) Comparison of odor-active compounds in grapes and wines from Vitis vinifera and non-foxy American grape species. Journal of agricultural and food chemistry 59 (19):10657-10664 
Sun Q, Gates MJ, Lavin EH, Acree TE, Sacks GL (2011b) Comparison of odor-active compounds in grapes and wines from vitis vinifera and non-foxy American grape species. J Agric Food Chem 59 (19):10657-10664. doi:10.1021/jf2026204

Sun Q, Sacks G, Lerch S, Heuvel JEV (2011c) Impact of shoot thinning and harvest date on yield components, fruit composition, and wine quality of Marechal Foch.

American journal of enology and viticulture 62 (1):32-41

Sweetman C, Deluc LG, Cramer GR, Ford CM, Soole KL (2009) Regulation of malate metabolism in grape berry and other developing fruits. Phytochemistry 70 (1112):1329-1344

Tautenhahn R, Patti GJ, Rinehart D, Siuzdak G (2012) XCMS Online: a web-based platform to process untargeted metabolomic data. Analytical chemistry 84 (11):5035-5039

Team RC (2019) R: A Language and Environment for Statistical Computing (Version 3.5. 2, R Foundation for Statistical Computing, Vienna, Austria, 2018). There is no corresponding record for this reference[Google Scholar]

Teh SL, Rostandy B, Awale M, Luby JJ, Fennell A, Hegeman AD (2019) Genetic analysis of stilbenoid profiles in grapevine stems reveals a major mQTL hotspot on chromosome 18 associated with disease-resistance motifs. Horticulture research $6(1): 1-11$

This P, Lacombe T, Thomas MR (2006) Historical origins and genetic diversity of wine grapes. Trends Genet 22 (9):511-519. doi:10.1016/j.tig.2006.07.008

Thomas AL, Harris JL, Bergmeier EA, Striegler RK (2017) Establishment Technique and Rootstock Impact 'Chambourcin'Grapevine Morphology and Production in Missouri. HortTechnology 27 (2):248-256

Toffali K, Zamboni A, Anesi A, Stocchero M, Pezzotti M, Levi M, Guzzo F (2011) Novel aspects of grape berry ripening and post-harvest withering revealed by untargeted LC-ESI-MS metabolomics analysis. Metabolomics 7 (3):424-436. doi:10.1007/s11306-010-0259-y

Tramontini S, Vitali M, Centioni L, Schubert A, Lovisolo C (2013) Rootstock control of scion response to water stress in grapevine. Environmental and experimental botany 93:20-26

Van Wyk C, Augustyn O, De Wet P, Joubert W (1979) Isoamyl acetate - a key fermentation volatile of wines of Vitis vinifera cv Pinotage. American Journal of Enology and Viticulture 30 (3):167-173

Villamor RR, Ross CF (2013) Wine matrix compounds affect perception of wine aromas. Annual review of food science and technology 4:1-20

Vivian Z, Thibaut V, Agnès D, Sandrine B, Fabrice L, Carole K, Katia G, Jorge ES, Olivier V, Jean-Laurent S (2018) The impact of plant water status on the gas exchange, berry composition and wine quality of Chasselas grapes in Switzerland. OENO One 52 (4). doi:10.20870/oeno-one.2018.52.4.2181

Vogt J, Schiller D, Ulrich D, Schwab W, Dunemann F (2013) Identification of lipoxygenase (LOX) genes putatively involved in fruit flavour formation in apple (Malus $\times$ domestica). Tree genetics \& genomes 9 (6):1493-1511

Vrhovsek U, Lotti C, Masuero D, Carlin S, Weingart G, Mattivi F (2014) Quantitative metabolic profiling of grape, apple and raspberry volatile compounds (VOCs) using a GC/MS/MS method. Journal of Chromatography B 966:132-139 
Wang Y, Chen W-K, Gao X-T, He L, Yang X-H, He F, Duan C-Q, Wang J (2019) Rootstock-Mediated Effects on Cabernet Sauvignon Performance: Vine Growth, Berry Ripening, Flavonoids, and Aromatic Profiles. International journal of molecular sciences 20 (2):401

Want E, Masson P (2011) Processing and analysis of GC/LC-MS-based metabolomics data. In: Metabolic profiling. Springer, pp 277-298

Warschefsky EJ, Klein LL, Frank MH, Chitwood DH, Londo JP, von Wettberg EJ, Miller AJ (2016) Rootstocks: diversity, domestication, and impacts on shoot phenotypes. Trends in plant science 21 (5):418-437

Winterhalter P, Rouseff R (2001) Carotenoid-Derived Aroma Compounds: An Introduction. In: Carotenoid-Derived Aroma Compounds, vol 802. ACS Symposium Series, vol 802. American Chemical Society, pp 1-17. doi:doi:10.1021/bk-2002-0802.ch001

10.1021/bk-2002-0802.ch001

Wooldridge J, Louw P, Conradie W (2010) Effects of rootstock on grapevine performance, petiole and must composition, and overall wine score of Vitis vinifera cv. Chardonnay and Pinot Noir. South African Journal of Enology and Viticulture 31 (1):45-48

Xia J, Wishart DS (2016) Using MetaboAnalyst 3.0 for Comprehensive Metabolomics Data Analysis. Current Protocols in Bioinformatics 55 (1):14.10.11-14.10.91. doi:10.1002/cpbi.11

Xu C-H, Chen G-S, Xiong Z-H, Fan Y-X, Wang X-C, Liu Y (2016) Applications of solid-phase microextraction in food analysis. TrAC Trends in Analytical Chemistry 80:12-29. doi:https://doi.org/10.1016/j.trac.2016.02.022

Young PR, Eyeghe-Bickong HA, du Plessis K, Alexandersson E, Jacobson DA, Coetzee Z, Deloire A, Vivier MA (2016) Grapevine plasticity in response to an altered microclimate: Sauvignon Blanc modulates specific metabolites in response to increased berry exposure. Plant physiology 170 (3):1235-1254

Yuan F, Qian MC (2016) Development of C13-norisoprenoids, carotenoids and other volatile compounds in Vitis vinifera L. Cv. Pinot noir grapes. Food chemistry 192:633-641

Zhang E, Chai F, Zhang H, Li S, Liang Z, Fan P (2017) Effects of sunlight exclusion on the profiles of monoterpene biosynthesis and accumulation in grape exocarp and mesocarp. Food chemistry 237:379-389

Zhang L, Marguerit E, Rossdeutsch L, Ollat N, Gambetta GA (2016) The influence of grapevine rootstocks on scion growth and drought resistance. Theoretical and Experimental Plant Physiology 28 (2):143-157. doi:10.1007/s40626-016-0070-x 


\section{Figures:}
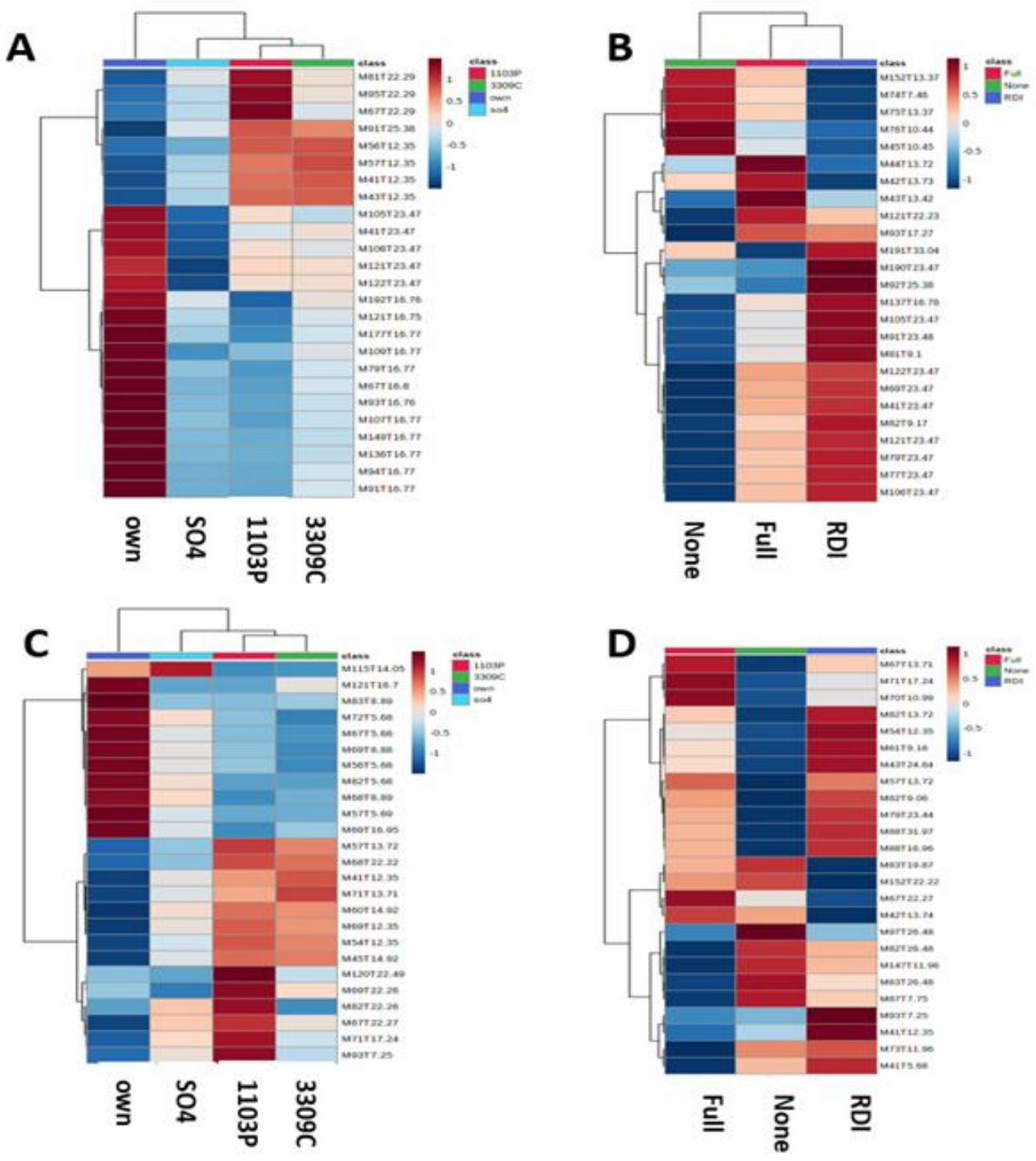

Figure 4.1: Heatmaps of the top 25 most influential features for differentiating (A) free volatiles by rootstocks, (B) free volatiles by irrigation regime, (C) total volatiles by rootstocks and (D) total volatiles by irrigation regimes. While only top contributors are shown the heatmaps were generated using all the features. The rows in the heatmap represent features $(\mathrm{M}(\mathrm{m} / \mathrm{z})$. T(time in minutes) and the columns indicate sample categories. The colors of the heatmap cells indicate the abundance of compounds across different samples. The color gradient, ranging from dark blue through white to dark red, represents low, middle and high abundance of a compound. 

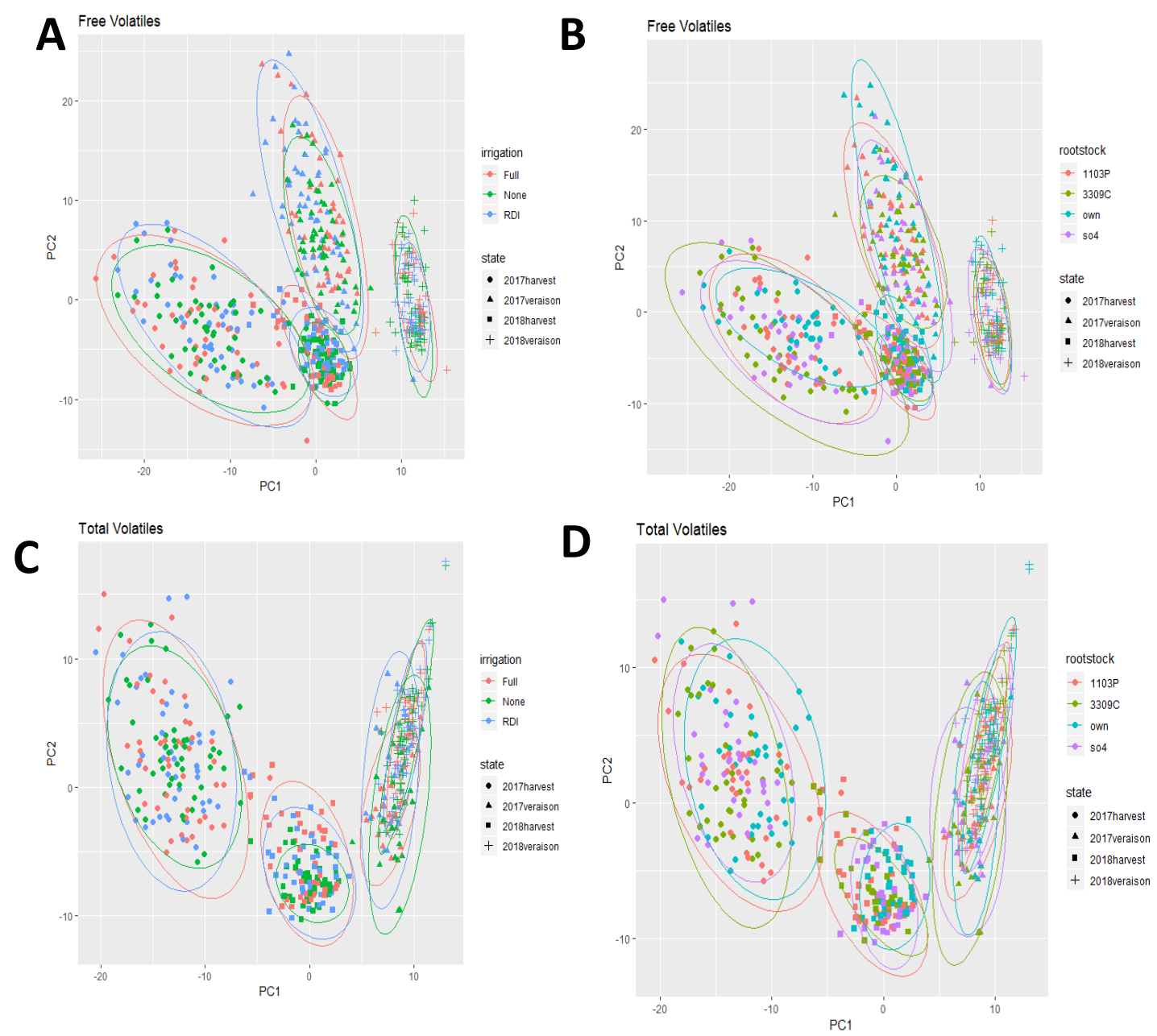

Figure 4.2: PCA Scores plot of the model obtained for free volatiles due to (A) irrigation regimes (B) rootstock and total volatiles due to (C) and rootstock D) detected by GC-MS. Samples collected from vines grafted with three different rootstocks and own-rooted Chambourcin subjected to three different levels of irrigation separated according to phenology and year. 


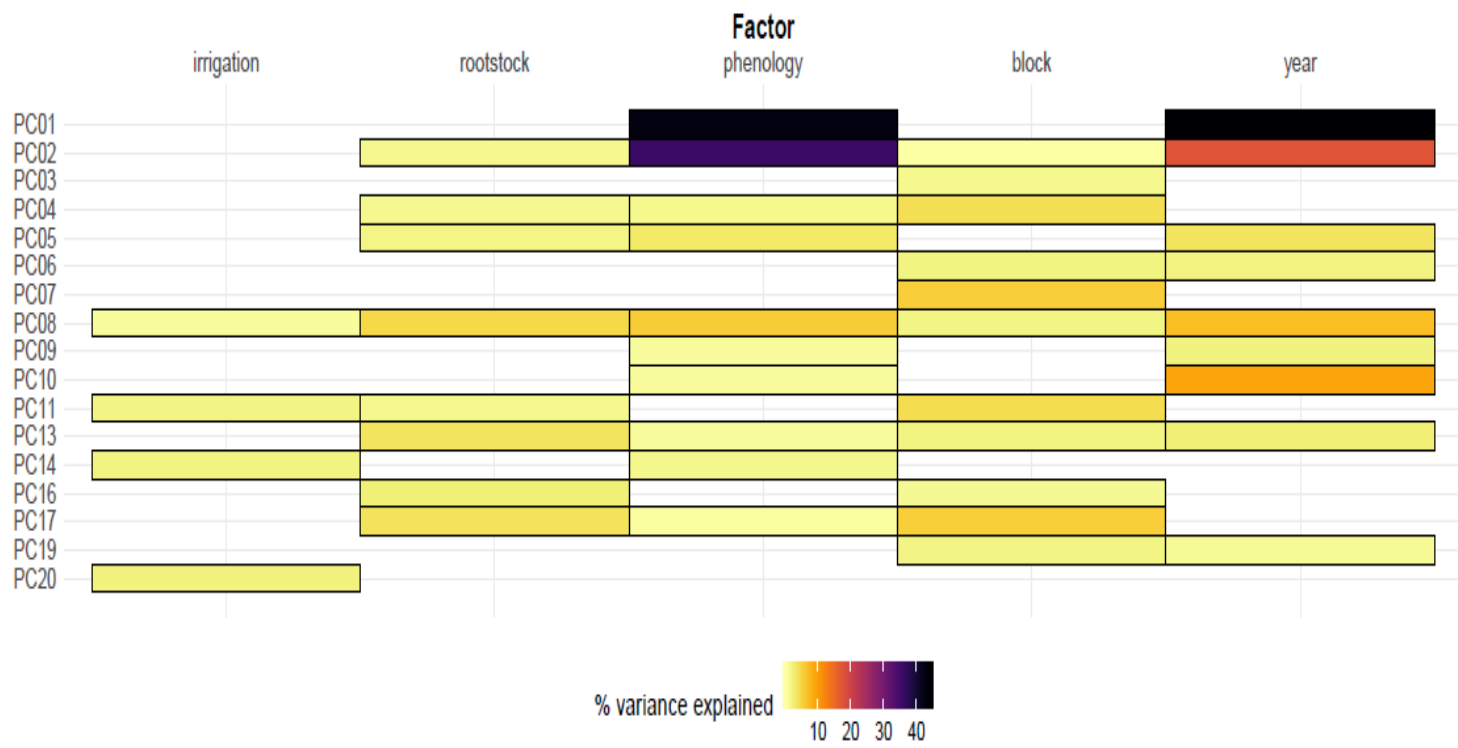

Figure 4.3:A linear model was estimated for free volatiles PCs 1 to 20. The model included irrigation (Full, RDI, None), rootstocks (1103P, 3309C, Own and SO4), phenology (veraison, harvest), block (based on the position in the vineyard), and year (2017 and 2018). The amount of variance explained by each PC is listed in the table S1 in parenthesis with the first 20 PCs capturing a total of $85.36 \%$ of the variation in free volatiles. Only the factors which explained a significant portion of variance $(\mathrm{p}<0.05)$ are plotted. The percent variance explained by each factor is indicated using color in the plot and also shown in table S1. 
PC1 and PC2 explained variation due to phenology and year.
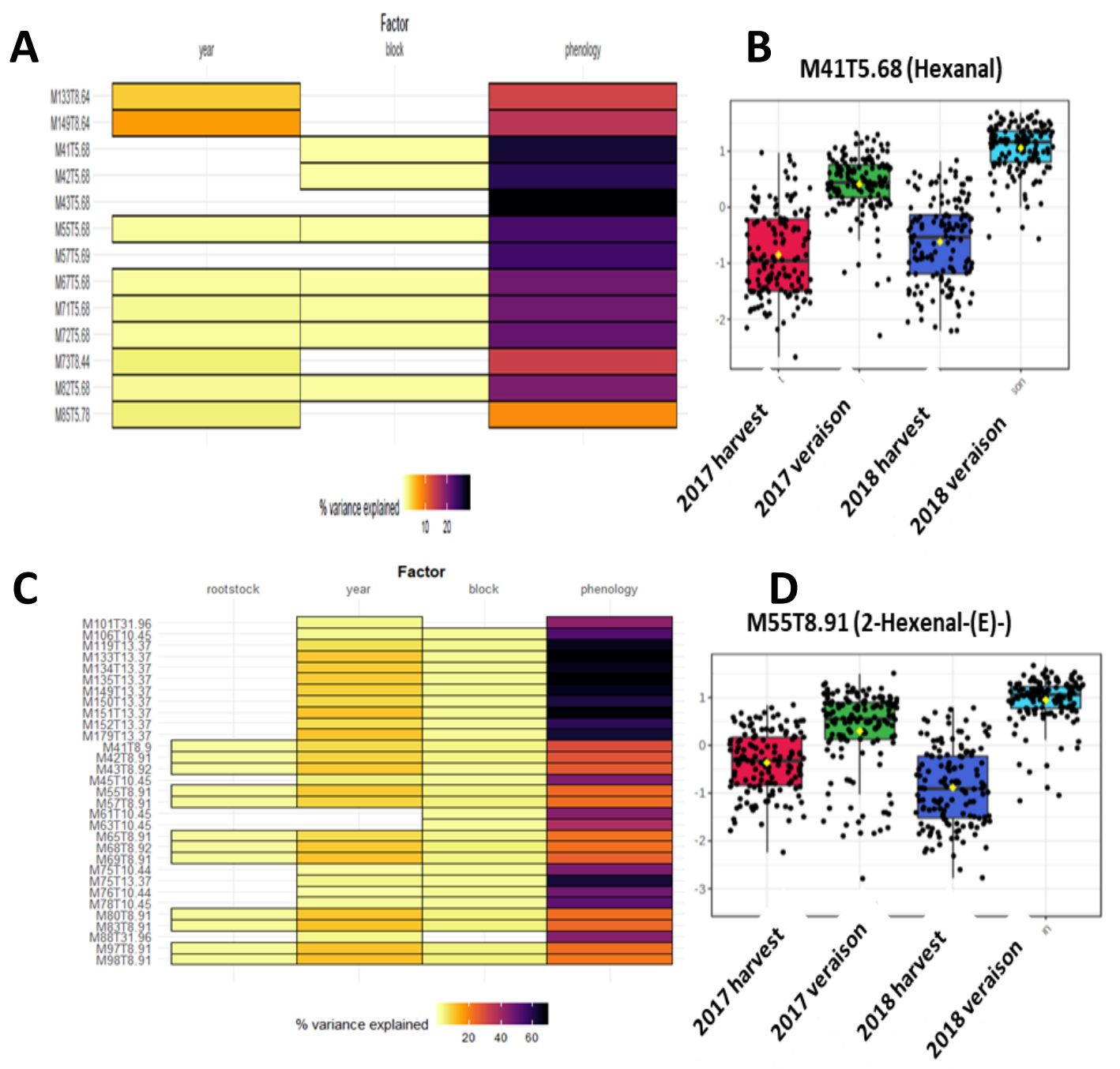

Figure 4.4 A linear model was fitted for (A) PC1 and (C) PC2 to extract features that are significant. Boxplot of a metabolite features differences between phenology in (B) PC1 and (D) PC2. The model included irrigation (Full, RDI, None), rootstocks (1103P, 3309C, Own and SO4), phenology (veraison, harvest), block (based on the the position in the vineyard), and year (2017 and 2018. Only the factors which explained a significant portion of variance $(p<0.05)$ are plotted. The percent variance explained by each factor is indicated using color in the plot. 

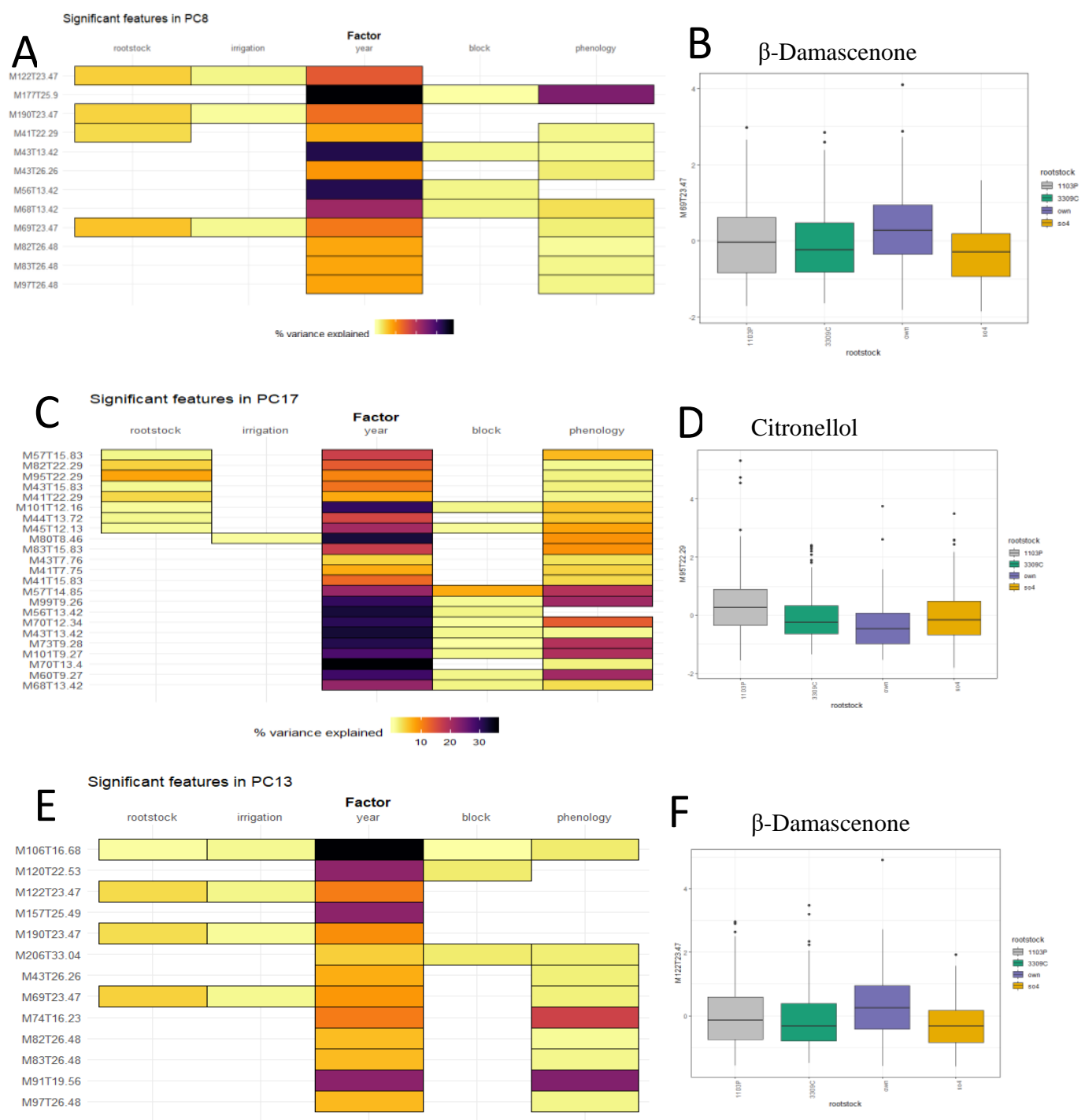

Significant features in $\mathrm{PC} 13$
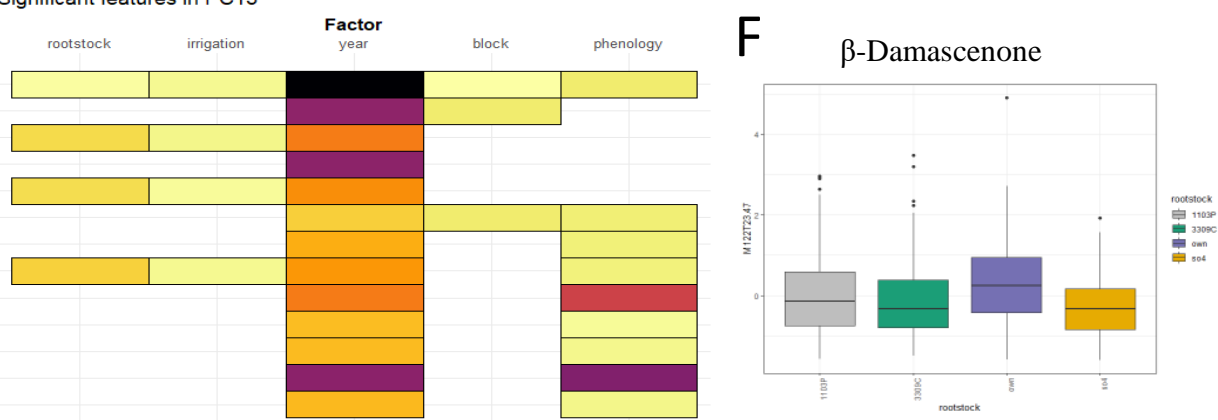

$\begin{array}{llllll}\% \text { variance explained } & 10 & 10 & 30 & 40\end{array}$

Figure 4.5 A linear model was fitted for (A) PC8, (C) PC17 and (E) PC13 to extract features that are significant. Boxplot of (B)PC8, (D) PC17 and (F)PC13 differences between different rootstocks for free volatiles. The model included irrigation (Full, RDI, None), rootstocks (1103P, 3309C, Own and SO4), phenology (veraison, harvest), block (based on the position in the vineyard), and year (2017 and 2018. Only the factors which explained a significant portion of variance $(\mathrm{p}<0.05)$ are plotted. The percent variance explained by each factor is indicated using color in the plot. 

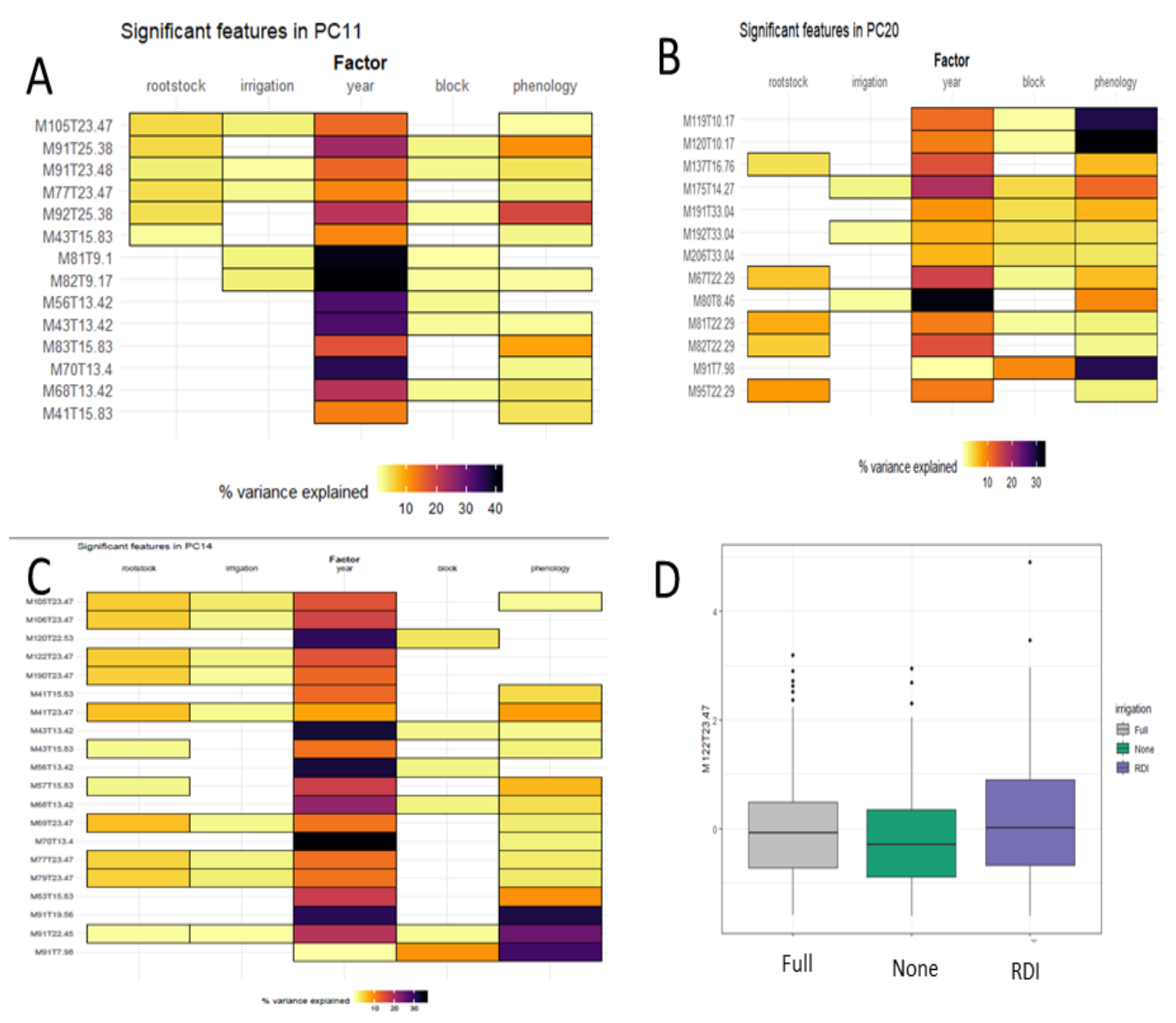

Figure 4.6: A linear model was fitted for (A) PC11, (B) PC20 and (C) PC14 respectively to extract features that are significant. D)Boxplot of one of the features indicating differences due to irrigation. The model included irrigation (Full, RDI, None), rootstocks (1103P, 3309C, Own and SO4), phenology (veraison, harvest), block (based on the position in the vineyard), and year (2017 and 2018. Only the factors which explained a significant portion of variance $(\mathrm{p}<0.05)$ are plotted. The percent variance explained by each factor is indicated using color in the plot. 


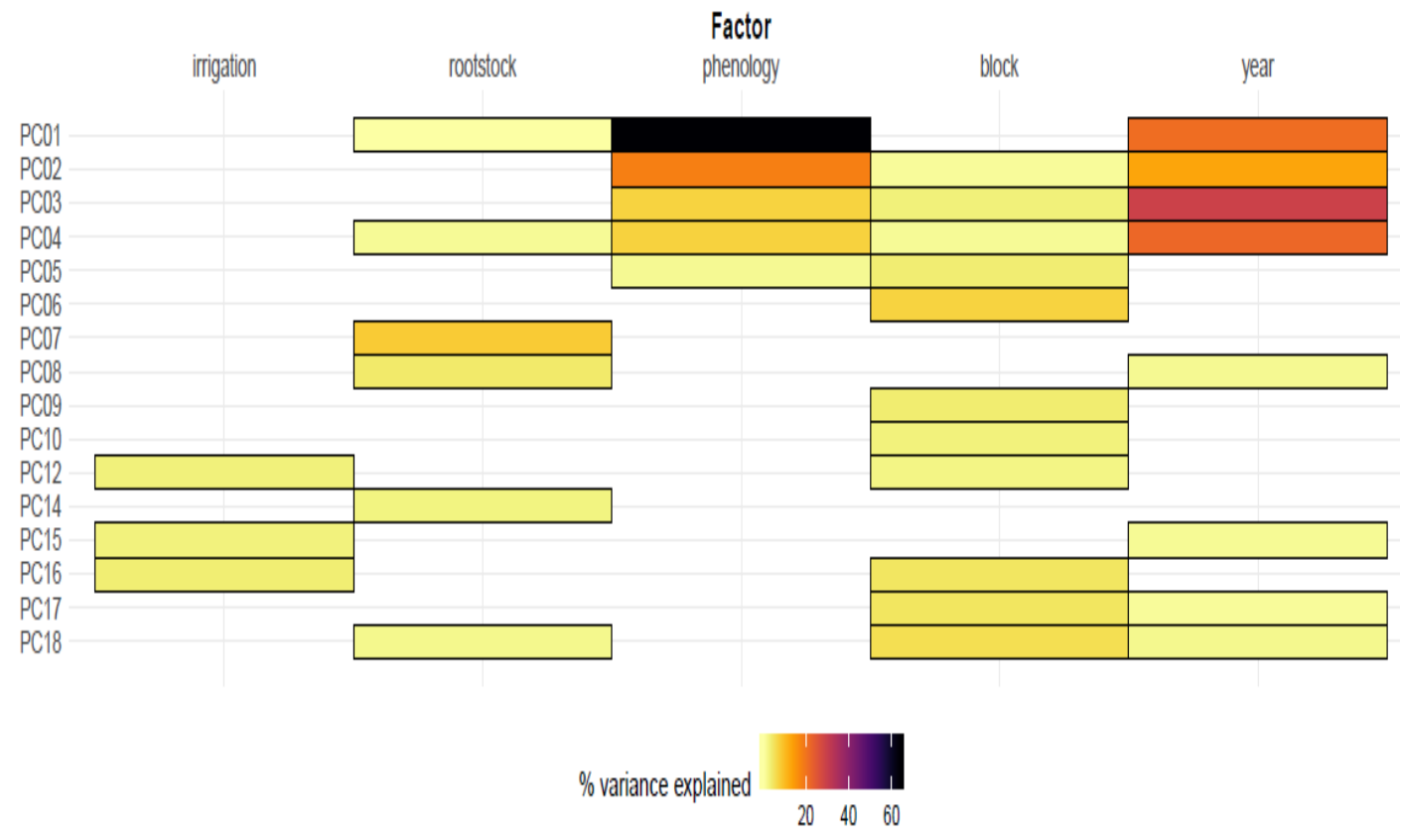

Figure 4.7: A linear model was estimated for total volatiles. The model included irrigation (Full, RDI, None), rootstocks (1103P, 3309C, Own and SO4), phenology (veraison, harvest), block (based on the position in the vineyard), and year (2017 and 2018). The amount of variance explained by each PC is listed in the table S1 in parenthesis with the first 20 PCs capturing a total of $85.68 \%$ of the variation in total volatiles. Only the factors which explained a significant portion of variance $(p<0.05)$ are plotted. The percent variance explained by each factor is indicated using color in the plot and also shown in table S1 
PCl explained variation in total volatiles due to phenology and year

A

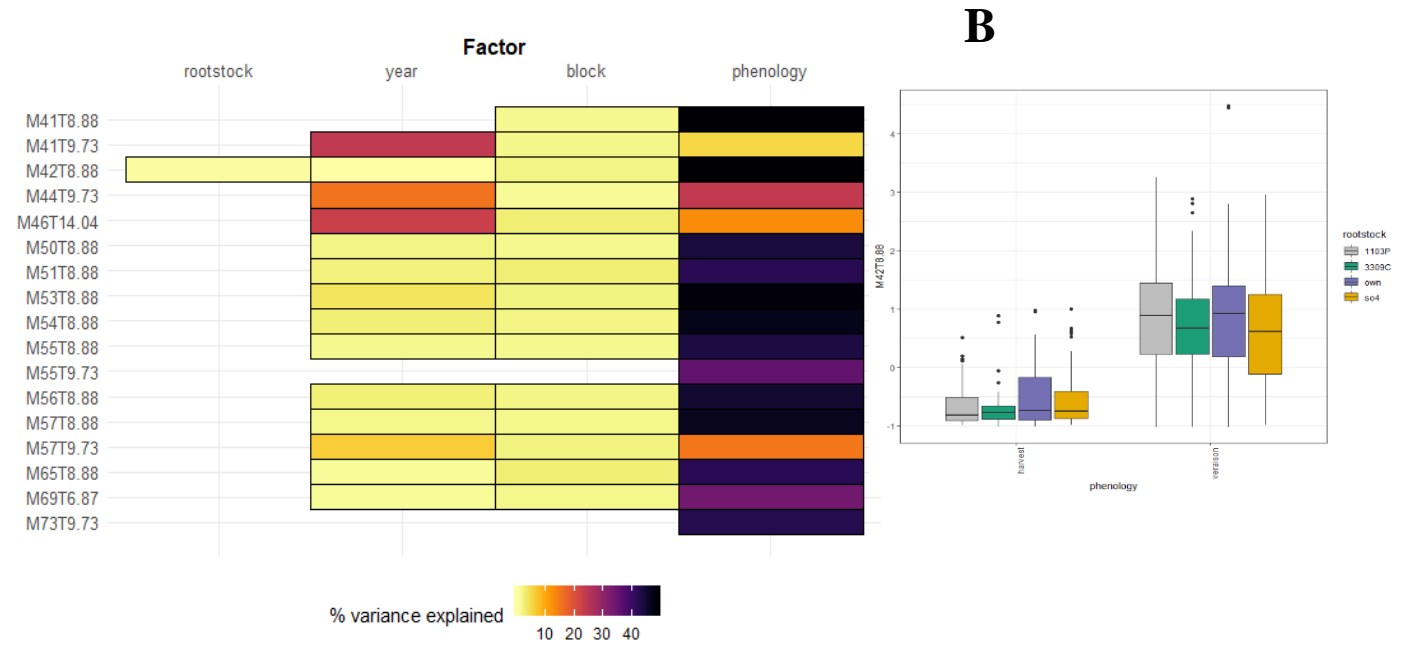

Figure 4.8:(A) A linear model was fitted for PC1 to extract features that are significant for phenology and year. The model included irrigation (Full, RDI, None), rootstocks (1103P, 3309C, Own and SO4), phenology (veraison, harvest), block (based on the position in the vineyard), and year (2017 and 2018). Only the factors which explained a significant portion of variance $(p<0.05)$ are plotted. The percent variance explained by each factor is indicated using color in the plot. (B) A boxplot showing a metabolite feature differences between veraison and harvest. 
PC4, PC7 and PC8 explained significant variation in total volatiles by Rootstocks:

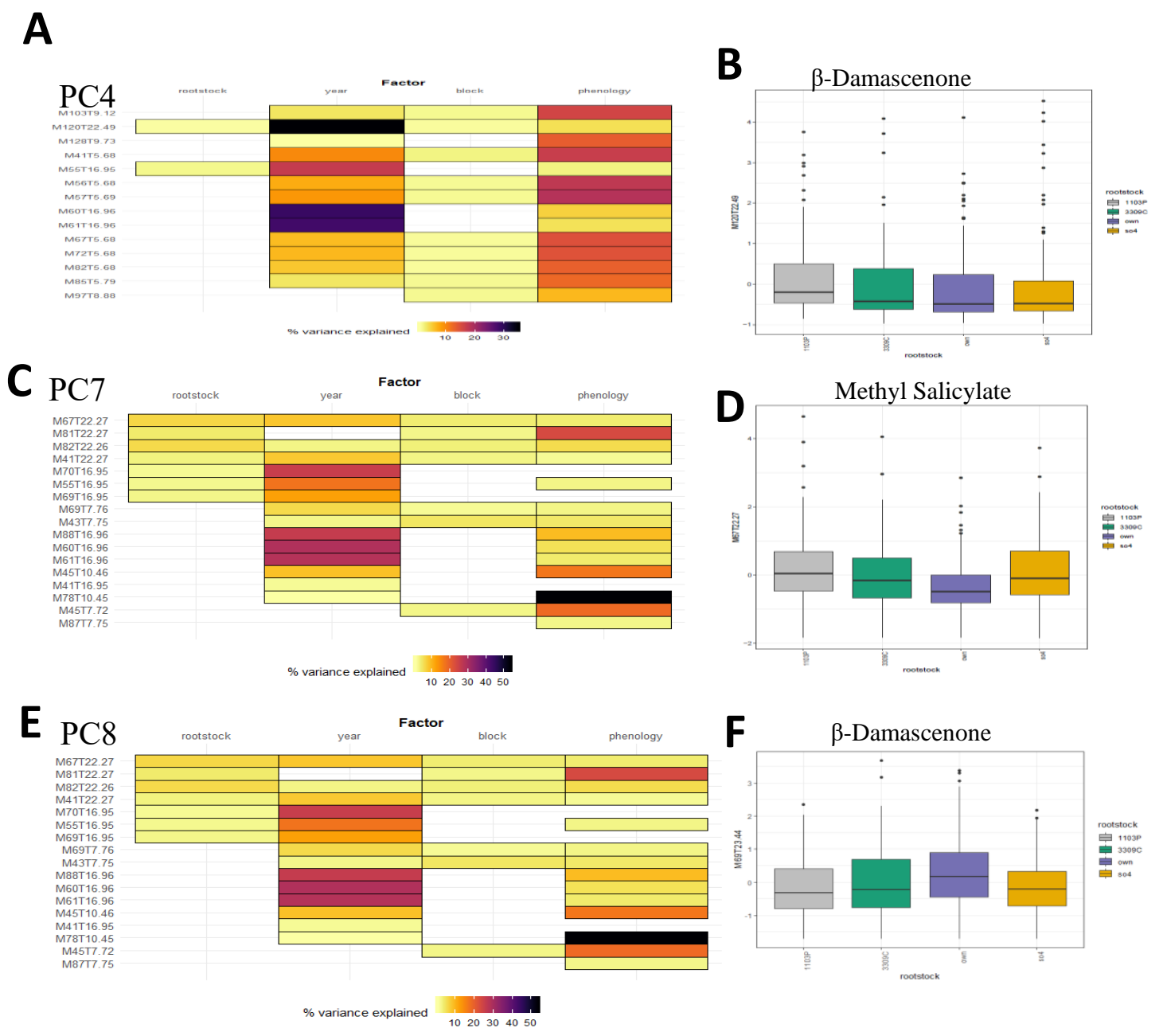

Figure 4.9: A linear model was fitted for(A) PC4, (C)PC7 and (E)PC8 respectively to extract features that are significant for rootstocks. On the right, boxplots showing differences in rootstocks due to (B)PC4, (D)PC7 and (F)PC8 respectively. The model included irrigation (Full, RDI, None), rootstocks (1103P, 3309C, Own and SO4), phenology (veraison, harvest), block (based on the position in the vineyard), and year (2017 and 2018). Only the factors which explained a significant portion of variance $(\mathrm{p}<0.05)$ are plotted. The percent variance explained by each factor is indicated using color in the plot. 


\section{Irrigation:}

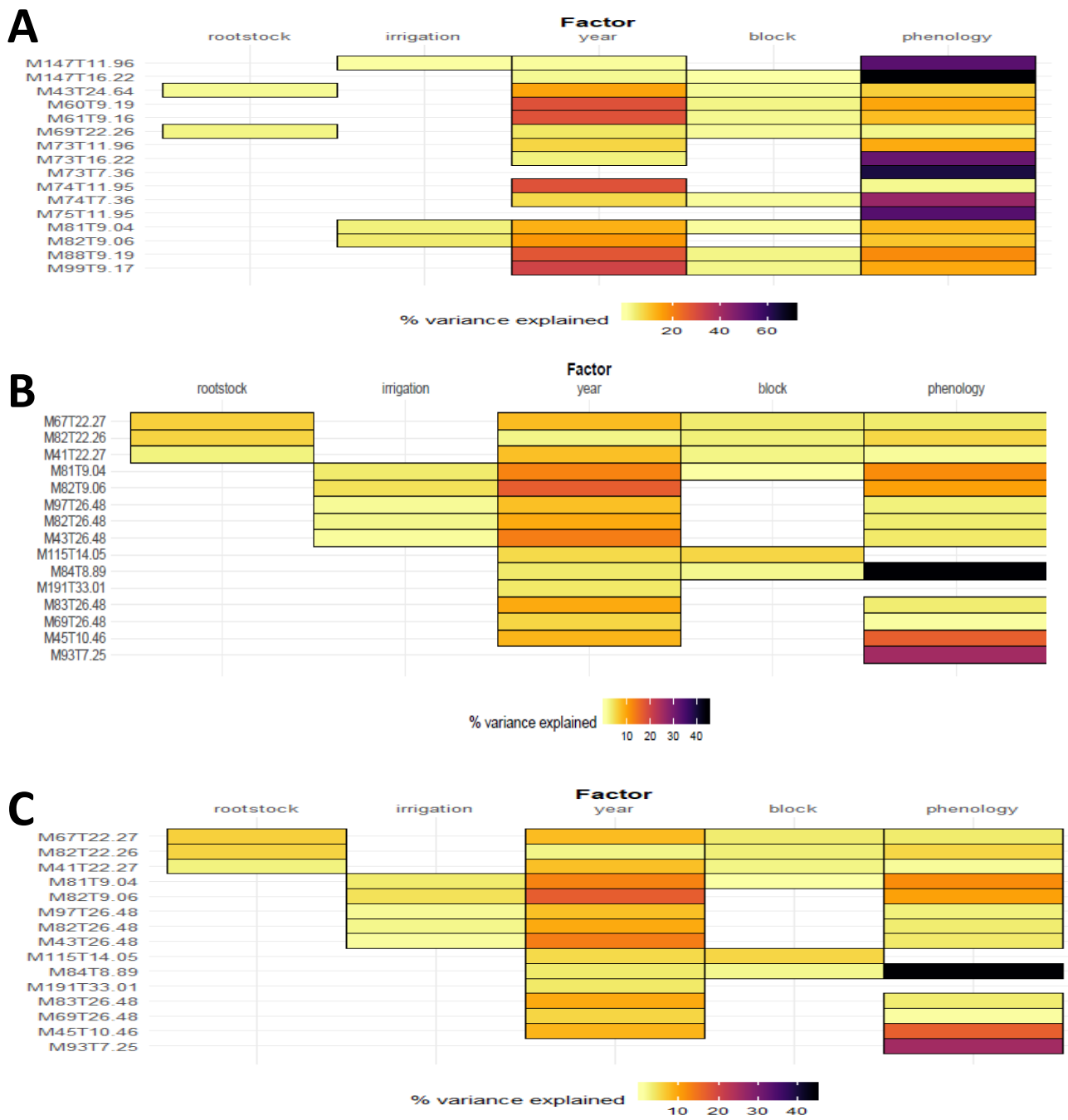

Figure 4.10: A linear model was fitted for (A) PC12, (B)PC16 and (C) PC15 respectively to extract features that are significant for rootstocks. The model included irrigation (Full, RDI, None), rootstocks (1103P, 3309C, Own and SO4), phenology (veraison, harvest), block (based on the position in the vineyard), and year (2017 and 2018). Only the factors which explained a significant portion of variance $(p<0.05)$ are plotted. The percent variance explained by each factor is indicated using color in the plot. 

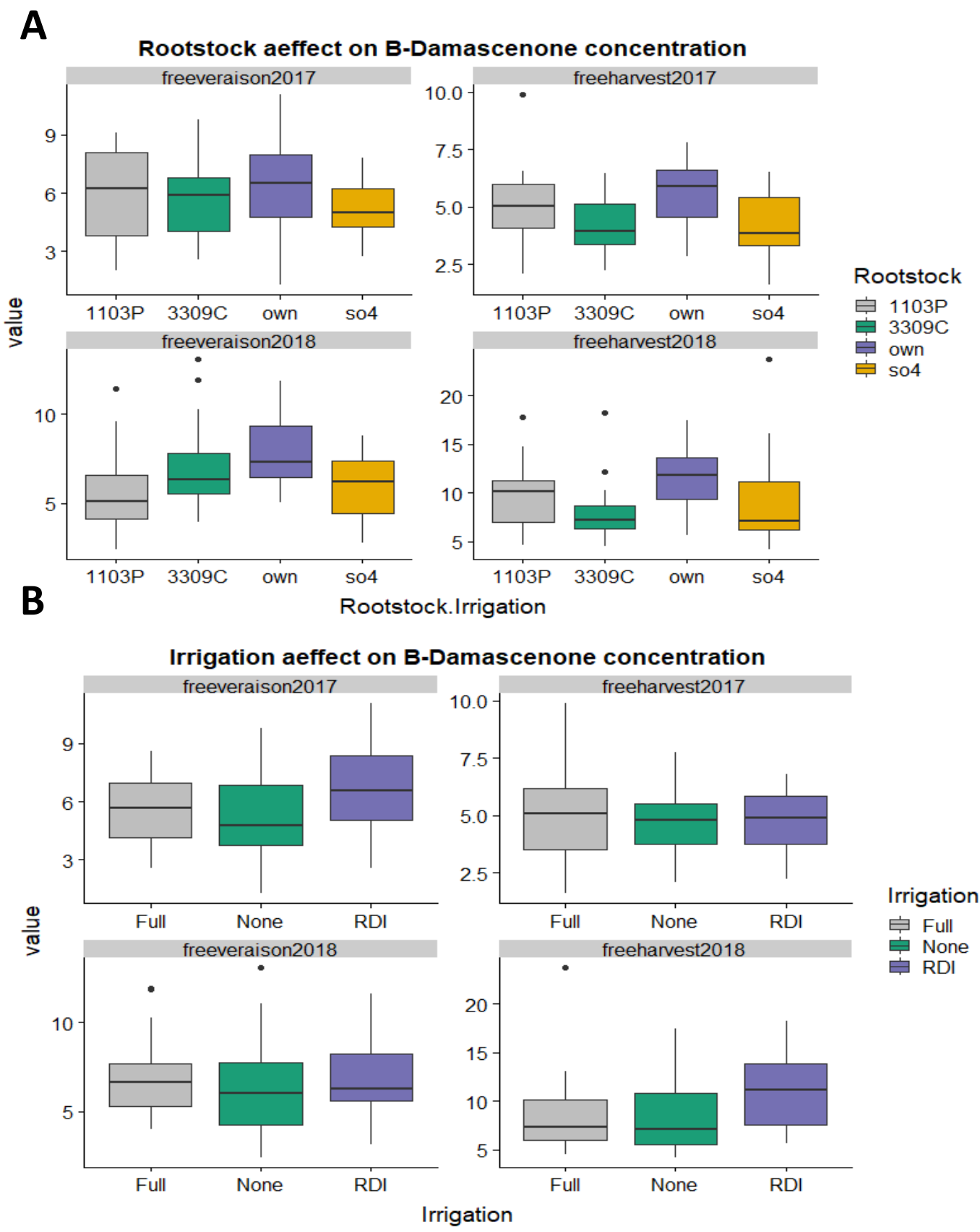

Figure 4.11 Boxplots showing the concentration of free $\beta$-Damascenone in different (A) rootstocks and $(\mathrm{B})$ irrigation regimes across various developmental stages and years. 

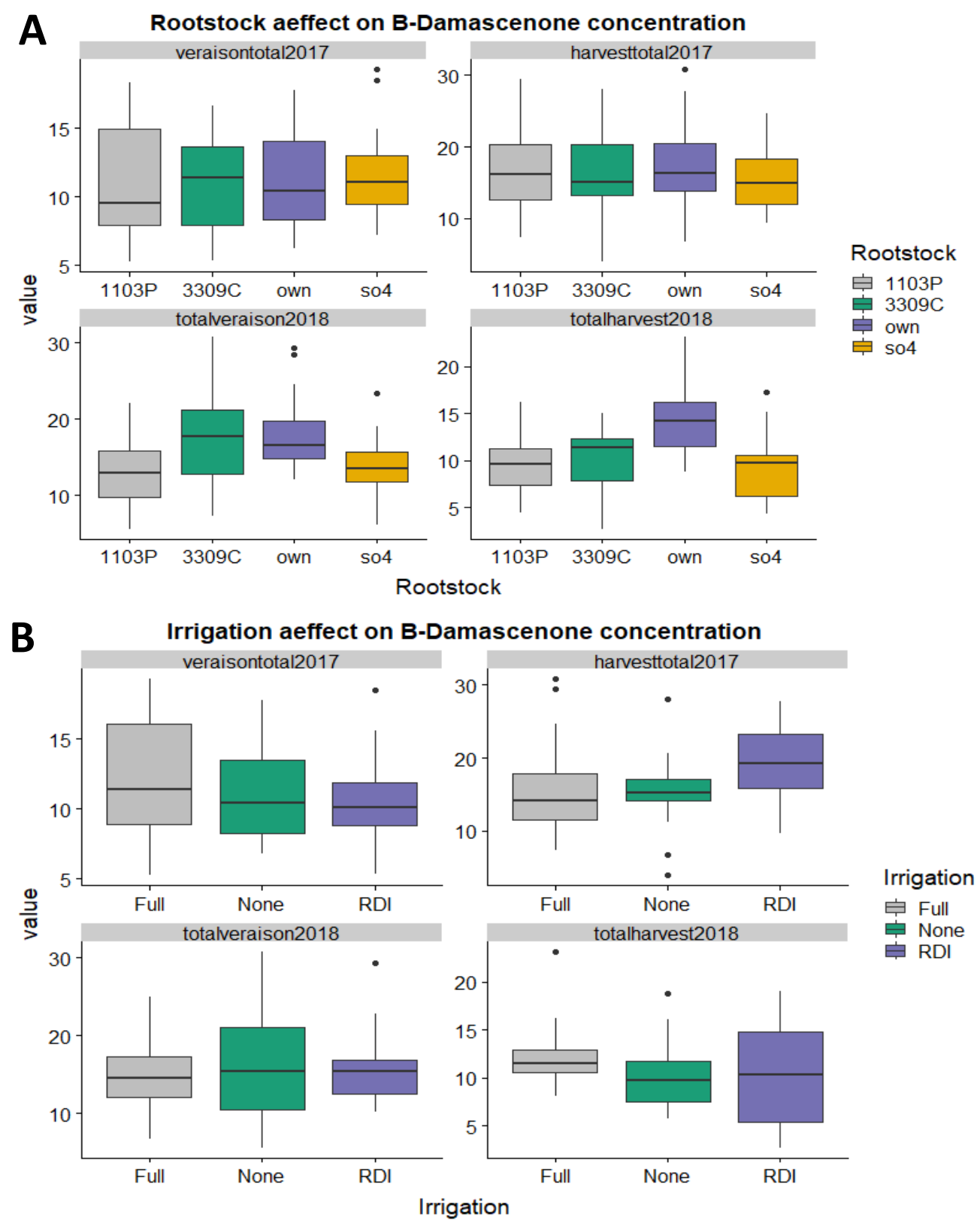

Figure 4.12:Boxplots showing the concentration of total $\beta$-Damascenone in different (A) rootstocks and (B) irrigation regimes across various developmental stages and years 


\section{Tables:}

Table 4.1: Mean concentrations of free, total and bound $\beta$-Damascenone in Chambourcin berries grafted to three different rootstocks and an own-rooted and subjected to three different irrigation regimes during two developmental stages for 2017 and 2018 seasons, Mt. Vernon, MO.

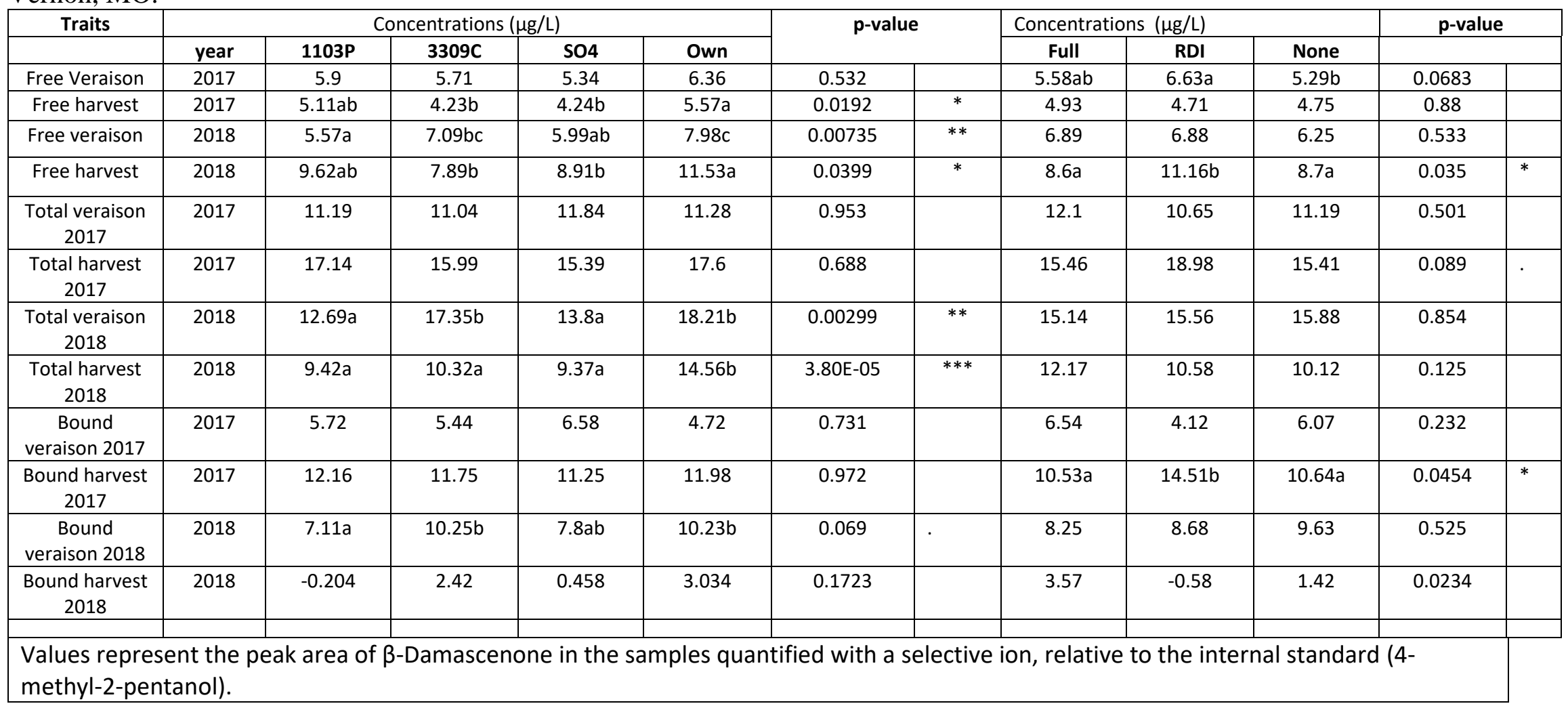


Analysis of variance was used to compare data with Rootstocks, Irrigation and Rootstock x Irrigation as factors; different letters within a row indicate significant differences for Duncan's Multiple Range test at $p<0.05$.

${ }^{*}$ means significant at $p<0.05,{ }^{* *}$ means significant at $p<0.01$ and ${ }^{* * *}$ means significance at $p<0.001$ 
Table 4.2 Mean relative concentrations of free, total and bound Methyl Salicylate in Chambourcin berries grafted to three different rootstocks and an own-rooted and subjected to three different irrigation regimes during two developmental stages for 2017 and, USA2018 seasons, Mt. Vernon, MO,USA.

\begin{tabular}{|c|c|c|c|c|c|c|c|c|c|c|c|c|}
\hline Traits & year & 1103P & 3309C & SO4 & Own & p-value & & Full & RDI & None & p-value & \\
\hline Free Veraison & 2017 & 1.81 & 1.5 & 1.97 & 1.76 & 0.791 & & 1.48 & 1.95 & 1.82 & 0.492 & \\
\hline Free harvest & 2017 & 0.91 & 0.62 & 0.83 & 0.81 & 0.516 & & 0.8 & 0.84 & 0.74 & 0.854 & \\
\hline Free veraison & 2018 & 1.81 & 1.48 & 1.99 & 1.84 & 0.731 & & 1.48 & 2.06 & 1.77 & 0.368 & \\
\hline Free harvest & 2018 & 0.78 & 0.51 & 0.5 & 0.42 & $4 \mathrm{E}-02$ & $* *$ & 0.55 & 0.53 & 0.57 & 0.89 & \\
\hline Total veraison 2017 & 2017 & $1.83 \mathrm{a}$ & $1.34 \mathrm{~b}$ & $1.44 \mathrm{~b}$ & $1.31 \mathrm{~b}$ & 0.615 & & 1.34 & 1.8 & 1.32 & 0.342 & \\
\hline Total harvest 2017 & 2017 & 1.27 & 0.96 & 1.08 & 1.08 & 0.668 & & 1.11 & 1.16 & 1.02 & 0.804 & \\
\hline Total veraison 2018 & 2018 & $4.39 \mathrm{a}$ & $2.43 \mathrm{~b}$ & $2.01 \mathrm{~b}$ & $1.96 \mathrm{~b}$ & $2 \mathrm{E}-07$ & $* * *$ & $2.33 \mathrm{a}$ & $3.29 \mathrm{~b}$ & $2.47 \mathrm{a}$ & 0.018 & * \\
\hline Total harvest 2018 & 2018 & $0.46 \mathrm{a}$ & $0.13 b$ & $0.18 \mathrm{~b}$ & $0.06 \mathrm{~b}$ & 0.00 & $* * *$ & 0.21 & 0.21 & 0.21 & 0.99 & \\
\hline
\end{tabular}

Values represent the peak area of Methyl Salicylate in the samples quantified with a selective ion, relative to the internal standard (2-Octanol).

Analysis of variance was used to compare data with Rootstocks, Irrigation and Rootstock x Irrigation as factors; different letters within a row indicate significant differences for Duncan's Multiple Range test at $p<0.05$.

$*$ means significant at $p<0.05, * *$ means significant at $p<0.01$ and $* * *$ means significance at $p<0.001$ 
Table 4.3: Metabolic Features that are predictive between harvest and veraison for free and total volatiles, as calculated as the correlation between harvest and veraison using Pearson's correlation. FDR correction was calculated to obtain the p-values.

\begin{tabular}{|c|c|c|c|c|c|}
\hline \multicolumn{6}{|c|}{ Free Volatiles } \\
\hline Harvest features & Veraison features & Compound Identified & $\begin{array}{l}\text { Pearson's } \\
\text { correlation } \\
\text { coefficient }\end{array}$ & $\mathrm{R} 2$ & $\mathrm{p}$-value \\
\hline H_2018M149T13.37 & V_2018M149T13.37 & 2-Hexen-1-ol,(E )- & 0.31 & 9.6 & 0 \\
\hline H_2018M83T13.4 & V_2018M83T13.4 & 2-Hexen-1-ol,(E )- & 0.30 & 9.0 & 0 \\
\hline H_2017M56T8.9 & V_2017M56T8.9 & 2-Hexenal,(E )- & 0.25 & 6.1 & $\begin{array}{c}1.9 \mathrm{E}- \\
02 \\
\end{array}$ \\
\hline H_2017M79T8.9 & V_2017M56T8.9 & 2-Hexenal,(E )- & 0.24 & 5.8 & $\begin{array}{c}2.4 \mathrm{E}- \\
02\end{array}$ \\
\hline H_2017M65T8.91 & V_2017M84T8.91 & 2-Hexenal,(E )- & 0.24 & 5.6 & $\begin{array}{c}2.7 \mathrm{E}- \\
02 \\
\end{array}$ \\
\hline H_2017M105T23.47 & V_2017M105T23.47 & B-Damascenone & 0.29 & 8.5 & $\begin{array}{c}3.7 \mathrm{E}- \\
03\end{array}$ \\
\hline H_2017M91T23.48 & V_2017M91T23.48 & B-Damascenone & 0.40 & 15.9 & $\begin{array}{c}1.3 \mathrm{E}- \\
05\end{array}$ \\
\hline H_2017M91T25.38 & V_2017M91T25.38 & phenylethyl alcohol & 0.35 & 12.0 & $\begin{array}{c}2.8 \mathrm{E}- \\
04\end{array}$ \\
\hline H_2017M92T25.38 & V_2017M92T25.38 & phenylethyl alcohol & 0.34 & 11.8 & $\begin{array}{c}3.3 \mathrm{E}- \\
04\end{array}$ \\
\hline \multicolumn{6}{|c|}{ Total Volatiles } \\
\hline H_2017_M88T9.19 & V_2017_M88T9.19 & Ethyl hexanoate & 0.41 & 16.8 & 0.01 \\
\hline H_2017_M61T9.16 & V_2017_M61T9.16 & Ethyl hexanoate & 0.38 & 14.4 & 0.02 \\
\hline H_2018_M177T16.73 & V_2018_M177T16.73 & Ethyl nononoate & 0.44 & 19.4 & 0 \\
\hline H_2018_M93T16.72 & V_2018_M93T16.72 & Ethyl nononoate & 0.40 & 16.0 & 0 \\
\hline H_2018_M192T16.73 & V_2018_M192T16.73 & Ethyl nononoate & 0.37 & 13.7 & 0 \\
\hline H_2018_M136T16.73 & V_2018_M136T16.73 & Ethyl nononoate & 0.37 & 13.7 & 0 \\
\hline H_2018_M121T16.7 & V_2018_M121T16.7 & Ethyl nononoate & 0.32 & 10.2 & 0 \\
\hline H_2018_M120T22.49 & V_2018_M120T22.49 & Methyl salicylate & 0.32 & 10.2 & 0 \\
\hline H_2018_M137T22.58 & V_2018_M137T22.58 & Methyl salicylate & 0.27 & 7.3 & 0.01 \\
\hline H_2018_M71T17.24 & V_2018_M71T17.24 & linalool & 0.26 & 6.8 & 0.01 \\
\hline H_2018_M82T22.26 & V_2018_M82T22.26 & citronellol & 0.25 & 6.3 & 0.02 \\
\hline
\end{tabular}

Correlations among veraison and harvest features was calculated using Pearson's correlation coefficient and FDR correction. Pairwise correlations between features with same RT that have Pearson's correlation coefficient $>0.23$ and $p$-value $<0.05$ were considered.

R2 was calculated as square of Pearson's correlation coefficient multiplied by 100 . Compounds were tentatively identified by comparing mass spectra of the samples with that of the NIST library, as well as by comparing the RT and mass spectra with authentic standards. 
Table 4. 4: Metabolic Features that are predictive between 2017 and 2018 for free and total volatiles, as calculated as correlation between 2017 and 2018 features using Pearson's correlation. FDR correction was calculated to obtain the p-values.

\begin{tabular}{|c|c|c|c|c|c|}
\hline \multicolumn{2}{|c|}{ 2017 Features } & \multirow{2}{*}{ 2018 Features } & Compound Identified & Pearson's & FDR \\
& & & Correlation & R2 & adjusted \\
& & & p-value \\
\hline H_2017M119T10.17 & H_2018M119T10.17 & o-cymene & 0.38 & 14.44 & 0 \\
\hline H_2017M120T10.17 & H_2018M120T10.17 & o-cymene & 0.32 & 10.24 & 0 \\
\hline H_2017M119T13.37 & H_2018M119T13.37 & 2-Hexen-1-ol, (Z)- & 0.34 & 11.56 & 0 \\
\hline H_2017M151T13.37 & H_2018M151T13.37 & 2-Hexen-1-ol, (Z)- & 0.33 & 10.89 & 0 \\
\hline H_2017M133T13.37 & H_2018M133T13.37 & 2-Hexen-1-ol, (Z)- & 0.32 & 10.24 & 0 \\
\hline H_2017M149T13.37 & H_2018M149T13.37 & 2-Hexen-1-ol, (Z)- & 0.3 & 9 & 0 \\
\hline H_2017M135T13.37 & H_2018M135T13.37 & 2-Hexen-1-ol, (Z)- & 0.3 & 9 & 0 \\
\hline H_2017M207T15 & H_2018M208T15 & 1-Octen-3-ol & 0.34 & 11.56 & 0 \\
\hline H_2017M208T15 & H_2018M208T15 & 1-Octen-3-ol & 0.3 & 9 & 0 \\
\hline H_2017M137T16.76 & H_2018M137T16.76 & Ethyl nononoate & 0.35 & 12.25 & 0 \\
\hline
\end{tabular}

Total Volatiles

\begin{tabular}{|c|c|c|c|c|c|}
\hline 2017H_M79T23.44 & 2018H_M79T23.44 & B-Damascenone & 0.25 & 6.25 & 0.02 \\
\hline 2017H_M77T23.44 & 2018H_M77T23.44 & B-Damascenone & 0.23 & 5.29 & 0.04 \\
\hline 2017H_M91T25.34 & 2018H_M91T25.34 & Phenylethyl Alcohol & 0.22 & 4.84 & 0.04 \\
\hline 2017H_M65T25.34 & 2018H_M65T25.34 & Phenylethyl Alcohol & 0.22 & 4.84 & 0.04 \\
\hline 2017H_M61T9.16 & 2018H_M61T9.16 & Ethyl hexanoate & 0.23 & 5.29 & 0.04 \\
\hline
\end{tabular}

Correlations among veraison and harvest features were calculated using Pearson's correlation coefficient and FDR correction. Pairwise correlations between features with the same RT that have Pearson's correlation coefficient $>0.23$ and $p$-value $<0.05$ were considered.

R2 was calculated as square of Pearson's correlation coefficient multiplied by 100.

Compounds were tentatively identified by comparing mass spectra of the samples with that of NIST library as well as by comparing RT and mass spectra with authentic standards. 


\section{Chapter 5: Rootstock and irrigation impact berry chemistry and organic acids.}

\section{Introduction:}

Flavor of grapes has been primarily attributed to the mix of sugars, acids, and volatiles. The chemical composition of harvested grapes strongly influences the composition of must at the onset of vinification and the final quality of the bottled wine (Mato et al. 2005). As grapes provide the basis for many wine aromas and flavors, the composition of berries at harvest and the factors affecting their composition are of much interest (Anesi et al. 2015). While volatiles and non-volatiles present in berry skin and flesh are important for the final wine quality, primary metabolites such as sugars and organic acids are the major indicators of ripening and determine any modifications required during winemaking as well as important for stability.

Sugars, $\mathrm{pH}$, and titratable acidity (TA) are the basic berry chemistry parameters used for determining the ripeness of berry. Sugars in grapes, mainly glucose and fructose are the primary sugars found in the grape berries and directly impact the final alcohol content of wine (Jackson 2008). Measured as soluble solids (in units of degrees Brix), glucose and fructose are predominant sugars in a Vitis vinifera berry, although the ratio of the two sugars may vary over the growing season or between cultivars (Kliewer 1965). Although present in trace amounts in most cultivars, sucrose has been measured at varying concentrations of total sugars in berries of interspecific hybrids (Liu et al. 2006). Sugar content in berries depends on various parameters such as vine vigor, rootstocks, crop load, vine water status, and a heat index of the growing season. 
In grape juice and wine, organic acid composition plays an important role in organoleptic properties, microbiological control, and wine stabilization (Kliewer et al. 1967). Organic acids produce refreshing sour taste but also modifies the perception of taste, mostly sweetness. The acids released from vacuoles during crushing leads to acid hydrolysis of aroma precursors, which causes the release of many volatile compounds such as monoterpenes, C13 norisoprenoids, benzyl alcohol, phenolics, and 2phenylethanol. The major organic acids in grape juice are tartaric acid and malic acid, which constitutes 70-90\% of total grape acidity (Kliewer et al. 1967; Ruffner 1982b), while citric acid and succinic acid is found in low proportion. Unlike other acids, tartaric acid is unique to grapes and is rarely subject to microbial metabolism (Mato et al. 2005). Tartaric acid accumulates in the berry skin and pulp before veraison, and its concentration per berry remains stable or slightly decreases throughout ripening in $\mathrm{V}$. vinifera (Kliewer et al. 1967). Malic acid, the most prevalent acid in apples, is high in the grape berry early in the season due to de novo synthesis of malate via glycolysis and the TCA cycle (Ruffner 1982a). Following veraison, there is a rapid decrease in malic acid in berries due to a significant decrease in malic acid synthesis and a sharp increase in malic acid degradation via respiration. In wine, malic acid is readily metabolized by malic acid bacteria such as Lactobacillus and Pediococcus $s p$ to lactic acid (Sweetman et al. 2009). This conversion of a diprotic acid to a monoprotic acid causes a drop in the perceived acidity of wine from a sensory perspective, which can further impact the perception of other volatile flavor and aroma compounds (Sweetman et al. 2009). This secondary malolactic (ML) fermentation is often associated with an increase in a buttery, vanilla, and creamy flavors and aromas that may be desirable and pleasant in certain 
wines, but can also be considered flaws if the flavors and aromas overwhelm the consumer's palate. For most winemakers, unless the desired wine profile is strongly influenced by ML characteristics, grapes are harvested at the lowest malic acid concentration possible for that cultivar, towards the end of the growing season.

Rootstocks and water stress are known to modify the composition of grapes and wine such as sugars, $\mathrm{pH}$, organic acids, phenolics, anthocyanins, and volatiles. Although primarily used for the phylloxera resistance, rootstocks play an important role in vine adaptation to various environmental conditions such as drought, salinity (Serra et al. 2014; Zhang et al. 2016). Rootstocks have a substantial influence on the physiology of the scion (Tramontini et al. 2013) as a consequence of genetic differences in root density, architecture signal and through their influence on vegetative growth, gas-exchange, water status, or nutrient uptake, rootstocks can influence yield and berry composition (Jones et al. 2009a; Koundouras et al. 2009; Nelson et al. 2015; JONES et al. 2009b; Koundouras et al. 2008). By affecting berry size and fruit chemistry parameters such as sugar content, organic acids, anthocyanins, and amino acids, rootstocks can impact the berry and wine volatiles, which are important quality determinant (Jones et al. 2009a; Harbertson and Keller 2011; Olarte Mantilla et al. 2018; Berdeja et al. 2014).

Most of the available information related to flavor in grapes and the factors impacting their composition has been investigated for Vitis vinifera grapes. Since, interspecific hybrid cultivars are known to have different berry chemistry than vinifera cultivars, understanding how the sugars and organic acids are impacted by rootstocks and irrigation treatments will help to produce wines with good quality. In this experiment, the 
impact of rootstocks and irrigation on organic acids and berry composition of Chambourcin grafted three different rootstocks and own-rooted and subjected to three irrigation regimes are studied.

\section{Materials and Methods:}

2.1 Experimental design and sampling and sample processing:

This study was conducted in an experimental vineyard at The University of Missouri Southwest Center Agricultural Experiment Station in Mount Vernon, Missouri, the USA during two seasons: 2017 and 2018. The experimental design is the same as that in Chapters 3 and 4. We sampled berries from 72 plants in the Chambourcin vineyard in 2017 and 2018 at harvest for berry chemistry (pH, titratable acid, soluble solids, organic acids) $(72=4$ rootstock combinations $\times 3$ replicates/vine $\times 3$ irrigation treatments $\times 2$ replicates/irrigation treatment). The berry samples were transported in dry ice to the lab and processed the next day.

\subsection{Midday Stem Water Potential:}

The water status was determined on the same day when other measurements were taken between $11 \mathrm{am}$ to $1 \mathrm{pm}$ by measuring the midday stem water potential using a pressure bomb/chamber (PMS Instrument Co., Albany, OR, USA). A single fully expanded, mature leaf per plant facing the sun was selected for the midday stem water potential measurements from each of two vines per treatment. Leaves were enclosed in an aluminum foil bag for at least 30 minutes before measurement, which prevents transpiration of the leaves and equilibrate with the water potential in stems to reflect the water potential of the xylem in the stem to which the leaf is attached. The leaves were 
placed into the pressure chamber with the petiole protruding from the chamber lid. The chamber was pressurized using a nitrogen tank, and the water potential was recorded when the initial xylem sap was observed emerging from the cut end of the petiole. The measurements from pressure bomb are expressed in bar or MPa, as a negative value.

\subsection{Berry Chemistry:}

In both 2017 and 2018, the samples collected during harvested were processed for basic chemical analysis and prepared for organic acid analysis using UPLC within 24 hours. Berries were homogenized using a Stomacher Model 400 circulator for a 30 second interval at $20^{\circ} \mathrm{C}$ (Seward, Worthington, West Sussex, UK). The homogenate was pressed manually through two layers of grade 40 cheesecloth and centrifuged at 10,000 rpm for 5 min (model 5804 R, Eppendorf AG, Hamburg). The $\mathrm{pH}$ of the juice samples was measured with a calibrated temperature compensating $\mathrm{pH}$ probe and Orion 3 star meter (Thermo Scientific, Fort Collins, CO). Aliquots (2ml) of juice were diluted to 50ml with degassed, deionized water, and titratable acidity was measured using a Compact Titrator (Model G20, Mettler-Toledo, Schwerzenbach, Switzerland) to an endpoint of 8.2 $\mathrm{pH}$, with $0.1 \mathrm{~N}$ sodium hydroxide $(\mathrm{NaOH})$ solution. Soluble solids percentage was determined using a temperature compensating digital refractometer (Reichert Technologies, Munich, Germany).

\subsection{Analysis of Organic acid concentrations in Berries using Reverse-Phase Ultra-}

\section{High-Performance Liquid Chromatography:}

For each harvest in both years, aliquots of $3 \mathrm{ml}$ of fresh juice were analyzed in duplicate analytical replicates for each treatment combination and each analytical 
replicate getting duplicate injections within 48 hours of sample collection. Samples were first decolorized with $0.5 \mathrm{~g}$ polyvinylpolypyrrolidone (PVPP) and in a $1.5 \mathrm{ml}$ centrifugal tubes, centrifuged at 14000 RPM for 5 minutes to remove anthocyanins from the juice. The supernatant was clarified through a $0.22 \mu \mathrm{m}$ filer (company name....) into duplicate $1.5 \mathrm{ml}$ glass vials and stored at $4^{\circ} \mathrm{C}$ until injection. Organic acids were analyzed using Waters UPLC (ACQUITY Waters Corp., UPLC H-Class, Milford, Mass.) system with a UV detector, and operated via Empower software (Version 3, Waters Corp., Milford, Mass.). A Zorbax SB-Aq column (4.6x250mm with $5 \mathrm{~mm}$ pore size) was operated at $35^{\circ} \mathrm{C}$ (Agilent Technologies, Santa Clara, Calif.). The aqueous mobile phase was isocratic with $99 \% 20 \mathrm{mM}$ sodium phosphate and $1 \%$ acetonitrile at a total flow rate of $0.5 \mathrm{ml}$ per minute (total run time of 8 minutes). The UV-visible lamp was set at $210 \mathrm{~nm}$ to detect tartaric, malic, and citric acid. Each acid was identified by reference standard and quantified with a 5-point calibration curve ranging from $0.25 \mathrm{~g} / \mathrm{L}-10 \mathrm{~g} / \mathrm{L}$. The sample injection volume was $1 \mu \mathrm{L}$.

\subsection{Statistical Analysis:}

To find statistical differences between rootstocks and irrigation regimes for the traits studied, ANOVA was performed followed by Duncan test in R 3.5.2 (Team 2019). Heatmaps were made using MetaboAnalyst (Xia and Wishart 2016).

\section{Results and Discussion:}

Rootstock and irrigation effects on basic grape maturity parameters (TSS, TA, and $\mathrm{pH}$ ) and organic acid content were determined. Heatmaps using averaged data from both years for all parameters showed that there are differences between berries from 
rootstocks and own-rooted (Fig 5.1). Titratable acidity (T.A.) and pH in 2017 and 2018 were significantly different between rootstocks as well as irrigation regimes $(\mathrm{p}<0.05)$ (Table 5.1). In both years, berries from own-rooted Chambourcin contained the lowest amounts of TA. Average total soluble solids (Brix) and tartaric acid are found to be highest in berries from own-rooted vines than other rootstocks, whereas, TA, $\mathrm{pH}$, malic acid, and citric acid were lowest in berries from own-rooted vines. Berries from ownrooted Chambourcin were found to have the lowest $\mathrm{pH}$ and TA than berries from other rootstocks (Fig 5.2) which is in contrast with (Harbertson and Keller 2011) where berries from own-rooted vines tended to have a higher $\mathrm{pH}$ than berries from other rootstocks. In the same study, no significant differences between rootstocks were found for sugar and TA in berries, but in wine anthocyanins and tannins. No significant differences were also observed between rootstocks and own-rooted Shiraz berries for TSS, TA, and pH (Olarte Mantilla et al. 2018). The lowest $\mathrm{pH}$ in the own-rooted Chambourcin berries in our study might be due to the presence of high malic acid. Chambourcin, being an interspecific hybrid known to have different berry chemistry than vinifera cultivars (Slegers et al. 2015; Slegers et al. 2017).

Irrigation does not have any significant effect on average TA and TSS content in berries, however, T.A. and TSS content are significantly different in 2018 but not in 2017 (Table 5.1). However, RDI is found to be significantly different for tartaric and malic acid content in berries, with RDI having a higher concentration of tartaric and malic acids in berries. Moderate water stress mediated through deficit irrigation has been found to have a positive impact on berry and wine quality by increasing the concentration of 
desired volatiles (Vivian et al. 2018; Reynolds et al. 2007; Romero et al. 2019; Qian et al. 2009).

Yearly variation in TA, TSS content, $\mathrm{pH}$, malic, and citric acid was found to be significant. The year 2017 contained significantly higher TS, TSS content, $\mathrm{pH}$, and lower malic acid in comparison to 2018 . The 2017 and 2018 seasons differed particularly in the amount and intensity of rainfall and temperature. Although total precipitation received in 2017 was higher than in 2018, 2017 received only four significant precipitation greater than 0.5 inches whereas 2018 received eight such significant precipitations. Likewise, the average temperature in 2017 is higher $(57.4 \mathrm{~F})$ than in $2018(55.7 \mathrm{~F})$, but the average temperature from harvest to veraison is higher in $2018(73.45 \mathrm{~F})$ than in $2017(70.36 \mathrm{~F})$. The yearly variation in rainfall and sun-exposed time has been found to be crucial in determining the composition of grapes as it impacts the photosynthesis and formation of many aroma compounds (Lee et al. 2009; Arapitsas et al. 2020; Schueuermann et al. 2016). The difference in rainfall has been found to be different in our study and thus has been a major driver of change in berry composition in our study.

Water potential is the measure of the water stress faced by the plant. Water potential is not significantly different between rootstocks in both years; however, it is found to be significantly different between irrigation regimes in 2017, but not in 2018 . Water potential of all rootstocks and irrigation is generally higher in 2017 in 2018, showing that the vines are generally more stressed in 2017 in comparison to 2018. This is also proved by the low soil moisture content in 2017 than in 2018. RDI vines had the 
highest water potential in 2017, however, in 2018 None vines had the highest water potential.

Yield per vine was not found to be significantly different according to rootstocks and irrigation for both years, however, the yield per vine is generally lower in 2017 in comparison to 2018. This is because, in 2017, some of the vines had very low yield due to bird damage and disease.

\section{Conclusion:}

Thus, we were able to measure the impact of rootstocks and irrigation regimes on basic berry chemistry traits such as TSS (Brix), pH, T.A., organic acids (tartaric acid, malic acid, and citric acid), and water potential of vines. While $\mathrm{pH}$, T.A, tartaric acid, and malic acid are significantly different due to rootstocks in both years, they are different only in one year or other due to irrigation. Total soluble sugar (Brix)is not significantly different due to rootstocks, but significant due to irrigation in 2018. Own-rooted berries had the lowest amount of $\mathrm{pH}$, T.A., and malic acid than rootstocks whereas, the highest amount of tartaric acid in both years and highest TSS content in 2017. Thus, rootstocks and irrigation have some effect on the basic berry chemistry traits and understanding these traits help manage various viticultural and winemaking decisions to make good quality wines as these traits directly impact the grapes and wine aroma and flavor.

\section{References:}

Alessandrini M, Battista F, Panighel A, Flamini R, Tomasi D (2017) Effect of pre-bloom leaf removal on grape aroma composition and wine sensory profile of Semillon cultivar. J Sci Food Agric. doi:10.1002/jsfa.8638 
Ali MB, Howard S, Chen S, Wang Y, Yu O, Kovacs LG, Qiu W (2011) Berry skin development in Norton grape: distinct patterns of transcriptional regulation and flavonoid biosynthesis. BMC plant biology 11 (1): 1

Ambers CP (2013) A historical hypothesis on the origin of the norton grape. Journal of Wine Research 24 (2):85-95. doi:10.1080/09571264.2012.747087

Ambers RK, Ambers CP (2004) Dr. Daniel Norborne Norton and the origin of the Norton grape. Am Wine Soc J 36 (3):77-87

Anesi A, Stocchero M, Dal Santo S, Commisso M, Zenoni S, Ceoldo S, Tornielli GB, Siebert TE, Herderich M, Pezzotti M (2015) Towards a scientific interpretation of the terroir concept: plasticity of the grape berry metabolome. BMC plant biology 15 (1): 191

Arapitsas P, Ugliano M, Marangon M, Piombino P, Rolle L, Gerbi V, Versari A, Mattivi F (2020) Use of Untargeted Liquid Chromatography-Mass Spectrometry Metabolome To Discriminate Italian Monovarietal Red Wines, Produced in Their Different Terroirs. Journal of Agricultural and Food Chemistry. doi:10.1021/acs.jafc.0c00879

Asproudi A, Petrozziello M, Cavalletto S, Guidoni S (2016) Grape aroma precursors in cv. Nebbiolo as affected by vine microclimate. Food chemistry 211:947-956

Balint G, Reynolds AG (2017) Irrigation level and time of imposition impact vine physiology, yield components, fruit composition and wine quality of Ontario Chardonnay. Scientia horticulturae 214:252-272

Barnuud NN, Zerihun A, Gibberd M, Bates B (2014) Berry composition and climate: responses and empirical models. International journal of biometeorology 58 (6):1207-1223

Battilana J, Costantini L, Emanuelli F, Sevini F, Segala C, Moser S, Velasco R, Versini G, Grando MS (2009) The 1-deoxy-d-xylulose 5-phosphate synthase gene colocalizes with a major QTL affecting monoterpene content in grapevine. Theoretical and Applied Genetics 118 (4):653-669

Berdeja M, Hilbert G, Dai ZW, Lafontaine M, Stoll M, Schultz HR, Delrot S (2014) Effect of water stress and rootstock genotype on Pinot Noir berry composition. Australian Journal of Grape and Wine Research 20 (3):409-421. doi:10.1111/ajgw.12091

Berdeja M, Nicolas P, Kappel C, Dai ZW, Hilbert G, Peccoux A, Lafontaine M, Ollat N, Gomès E, Delrot S (2015) Water limitation and rootstock genotype interact to alter grape berry metabolism through transcriptome reprogramming. Horticulture research 2:15012

Bianco RL, Farina V, Avellone G, Filizzola F, Agozzino P (2008) Fruit quality and volatile fraction of 'Pink Lady'apple trees in response to rootstock vigor and partial rootzone drying. Journal of the Science of Food and Agriculture 88 (8):1325-1334

Biasoto ACT, Netto FM, Marques EJN, da Silva MAAP (2014) Acceptability and preference drivers of red wines produced from Vitis labrusca and hybrid grapes. Food Research International 62:456-466. doi:10.1016/j.foodres.2014.03.052

Bindon KA, Dry PR, Loveys BR (2007) Influence of Plant Water Status on the Production of C13-Norisoprenoid Precursors in Vitis vinifera L. Cv. Cabernet 
Sauvignon Grape Berries. Journal of Agricultural and Food Chemistry 55

(11):4493-4500. doi:10.1021/jf063331p

Bisson LF, Waterhouse AL, Ebeler SE, Walker MA, Lapsley JT (2002a) The present and future of the international wine industry. Nature 418 (6898):696

Bisson LF, Waterhouse AL, Ebeler SE, Walker MA, Lapsley JT (2002b) The present and future of the international wine industry. Nature 418 (6898):696-699. doi:10.1038/nature01018

Black C, Parker M, Siebert T, Capone D, Francis I (2015) Terpenoids and their role in wine flavour: recent advances. Australian journal of grape and wine research 21:582-600

Callejon RM, Margulies B, Hirson GD, Ebeler SE (2012) Dynamic Changes in Volatile Compounds during Fermentation of Cabernet Sauvignon Grapes with and without Skins. American Journal of Enology and Viticulture 63 (3):301-312. doi:10.5344/ajev.2012.12009

Canuti V, Conversano M, Calzi ML, Heymann H, Matthews MA, Ebeler SE (2009) Headspace solid-phase microextraction-gas chromatography-mass spectrometry for profiling free volatile compounds in Cabernet Sauvignon grapes and wines. J Chromatogr A 1216 (15):3012-3022. doi:10.1016/j.chroma.2009.01.104

Carlin S, Masuero D, Guella G, Vrhovsek U, Mattivi F (2019) Methyl Salicylate Glycosides in Some Italian Varietal Wines. Molecules 24 (18). doi:10.3390/molecules24183260

Carrasco-Quiroz M, Martínez-Gil AM, Gutiérrez-Gamboa G, Moreno-Simunovic Y (2020) Effect of rootstocks on volatile composition of Merlot wines. Journal of the Science of Food and Agriculture 100 (8):3517-3524. doi:10.1002/jsfa.10395

Chapman DM, Roby G, Ebeler SE, Guinard JX, Matthews MA (2005) Sensory attributes of Cabernet Sauvignon wines made from vines with different water status. Australian Journal of Grape and Wine Research 11 (3):339-347

Chaves MM, Santos TP, Souza Cd, Ortuño M, Rodrigues M, Lopes C, Maroco J, Pereira JS (2007) Deficit irrigation in grapevine improves water-use efficiency while controlling vigour and production quality. Annals of Applied Biology 150 (2):237-252

Chisholm MG, Guiher LA, Vonah TM, Beaumont JL (1994) Comparison of some French-American hybrid wines with White Riesling using gas chromatographyolfactometry. American journal of enology and viticulture 45 (2):201-212

Commisso M, Strazzer P, Toffali K, Stocchero M, Guzzo F (2013) Untargeted metabolomics: an emerging approach to determine the composition of herbal products. Computational and structural biotechnology journal 4 (5):e201301007

Cookson SJ, Ollat N (2013) Grafting with rootstocks induces extensive transcriptional reprogramming in the shoot apical meristem of grapevine. BMC Plant Biology 13 (1):147. doi:10.1186/1471-2229-13-147

Coombe B, BOVIO M, SCHNEIDER A (1987) Solute accumulation by grape pericarp cells: v. relationship to berry size and the effects of defoliation. Journal of Experimental Botany 38 (11):1789-1798

CooMbe BG, McCarthy M (2000) Dynamics of grape berry growth and physiology of ripening. Australian journal of grape and wine research 6 (2):131-135 
Cousins P (2005) Evolution, genetics, and breeding: viticultural applications of the origins of our rootstocks. Grapevine Rootstocks: Current Use, Research, and Application: 1

Cuadros-Inostroza A, Giavalisco P, Hummel J, Eckardt A, Willmitzer L, Peña-Cortés H (2010) Discrimination of wine attributes by metabolome analysis. Analytical Chemistry 82 (9):3573-3580

Cuadros-Inostroza A, Ruíz-Lara S, González E, Eckardt A, Willmitzer L, Peña-Cortés H (2016) GC-MS metabolic profiling of Cabernet Sauvignon and Merlot cultivars during grapevine berry development and network analysis reveals a stage-and cultivar-dependent connectivity of primary metabolites. Metabolomics 12 (2):39

de Pinho PG, Bertrand A (1995) Analytical determination of furaneol (2, 5-dimethyl-4hydroxy-3 (2H)-furanone). Application to differentiation of white wines from hybrid and various Vitis vinifera cultivars. American journal of enology and viticulture 46 (2):181-186

De Vos RC, Moco S, Lommen A, Keurentjes JJ, Bino RJ, Hall RD (2007) Untargeted large-scale plant metabolomics using liquid chromatography coupled to mass spectrometry. Nature protocols 2 (4):778

Deluc LG, Quilici DR, Decendit A, Grimplet J, Wheatley MD, Schlauch KA, Mérillon JM, Cushman JC, Cramer GR (2009) Water deficit alters differentially metabolic pathways affecting important flavor and quality traits in grape berries of Cabernet Sauvignon and Chardonnay. BMC genomics 10 (1):212

Dempsey DMA, Vlot AC, Wildermuth MC, Klessig DF (2011) Salicylic Acid biosynthesis and metabolism. The arabidopsis book 9:e0156-e0156. doi:10.1199/tab.0156

des Gachons CP, Leeuwen CV, Tominaga T, Soyer JP, Gaudillère JP, Dubourdieu D (2005) Influence of water and nitrogen deficit on fruit ripening and aroma potential of Vitis vinifera L cv Sauvignon blanc in field conditions. Journal of the Science of Food and Agriculture 85 (1):73-85

Doligez A, Audiot E, Baumes R, This P (2006) QTLs for muscat flavor and monoterpenic odorant content in grapevine (Vitis vinifera L.). Molecular Breeding 18 (2):109-125

Dunemann F, Ulrich D, Boudichevskaia A, Grafe C, Weber W (2009) QTL mapping of aroma compounds analysed by headspace solid-phase microextraction gas chromatography in the apple progeny 'Discovery' $\times$ 'Prima'. Molecular breeding 23 (3):501-521

Ebeler SE (2001) Analytical Chemistry: Unlocking the Secrets of Wine Flavor. Food Reviews International 17 (1):45-64. doi:10.1081/fri-100000517

Ebeler SE, Thorngate JH (2009) Wine chemistry and flavor: looking into the crystal glass. J Agric Food Chem 57 (18):8098-8108. doi:10.1021/jf9000555

El-Ansary DO, Okamoto G (2007) Vine Water Relations and Quality of \&lsquo;Muscat of Alexandria\&rsquo; Table Grapes Subjected to Partial Root-zone Drying and Regulated Deficit Irrigation. Journal of the Japanese Society for Horticultural Science 76 (1):13-19. doi:10.2503/jjshs.76.13

Escudero A, Campo E, Fariña L, Cacho J, Ferreira V (2007) Analytical characterization of the aroma of five premium red wines. Insights into the role of odor families and 
the concept of fruitiness of wines. Journal of Agricultural and Food Chemistry 55 (11):4501-4510

Ezzahouani A, Williams LE (1995) The influence of rootstock on leaf water potential, yield, and berry composition of Ruby Seedless grapevines. American Journal of Enology and Viticulture 46 (4):559-563

Fang Y, Qian MC (2006) Quantification of selected aroma-active compounds in Pinot noir wines from different grape maturities. Journal of agricultural and food chemistry 54 (22):8567-8573

Fang Y, Qian MC (2012) Development of C6 and Other Volatile Compounds in Pinot Noir Grapes Determined by Stir Bar Sorptive Extraction-GC-MS. In: Flavor Chemistry of Wine and Other Alcoholic Beverages, vol 1104. ACS Symposium Series, vol 1104. American Chemical Society, pp 81-99. doi:doi:10.1021/bk2012-1104.ch006

10.1021/bk-2012-1104.ch006

Ferreira V, Lopez R (2019) The Actual and Potential Aroma of Winemaking Grapes. Biomolecules 9 (12):818

Ferreira V, López R, Cacho JF (2000) Quantitative determination of the odorants of young red wines from different grape varieties. Journal of the Science of Food and Agriculture 80 (11):1659-1667

Ferris H, Zheng L, Walker M (2012) Resistance of grape rootstocks to plant-parasitic nematodes. Journal of nematology 44 (4):377

Flamini R, De Rosso M, Panighel A, Dalla Vedova A, De Marchi F, Bavaresco L (2014) Profiling of grape monoterpene glycosides (aroma precursors) by ultra-high performance-liquid chromatography-high resolution mass spectrometry (UHPLC/QTOF). J Mass Spectrom 49 (12):1214-1222. doi:10.1002/jms.3441

Francis I, Newton J (2005) Determining wine aroma from compositional data. Australian Journal of Grape and Wine Research 11 (2):114-126

Fung RW, Gonzalo M, Fekete C, Kovacs LG, He Y, Marsh E, McIntyre LM, Schachtman DP, Qiu W (2008) Powdery mildew induces defense-oriented reprogramming of the transcriptome in a susceptible but not in a resistant grapevine. Plant physiology 146 (1):236-249

Galletto L, Barisan L, Boatto V, AC Costantini E, Lorenzetti R, Pomarici E, Vecchio R (2014) More crop for drop-climate change and wine: an economic evaluation of a new drought-resistant rootstock. Recent patents on food, nutrition \& agriculture 6 (2):100-112

Ghaste M, Narduzzi L, Carlin S, Vrhovsek U, Shulaev V, Mattivi F (2015) Chemical composition of volatile aroma metabolites and their glycosylated precursors that can uniquely differentiate individual grape cultivars. Food Chem 188:309-319. doi:10.1016/j.foodchem.2015.04.056

Gika HG, Theodoridis GA, Plumb RS, Wilson ID (2014) Current practice of liquid chromatography-mass spectrometry in metabolomics and metabonomics. J Pharm Biomed Anal 87:12-25. doi:10.1016/j.jpba.2013.06.032 
Gouthu S, Deluc LG (2015) Timing of ripening initiation in grape berries and its relationship to seed content and pericarp auxin levels. BMC plant biology 15 (1):46

Gowda H, Ivanisevic J, Johnson CH, Kurczy ME, Benton HP, Rinehart D, Nguyen T, Ray J, Kuehl J, Arevalo B, Westenskow PD, Wang J, Arkin AP, Deutschbauer AM, Patti GJ, Siuzdak G (2014) Interactive XCMS Online: Simplifying Advanced Metabolomic Data Processing and Subsequent Statistical Analyses. Analytical Chemistry 86 (14):6931-6939. doi:10.1021/ac500734c

Gross JH (2006) Mass spectrometry: a textbook. Springer Science \& Business Media, Gu S, Read PE, Gamet S (2005) Performance of 'Gewurztraminer'on six rootstocks under marginal climatic conditions. Grapevine Rootstocks: Current Use, Research, and Application:57

Guillaumie S, Ilg A, Réty S, Brette M, Trossat-Magnin C, Decroocq S, Léon C, Keime C, Ye T, Baltenweck-Guyot R (2013) Genetic analysis of the biosynthesis of 2methoxy-3-isobutylpyrazine, a major grape-derived aroma compound impacting wine quality. Plant physiology 162 (2):604-615

Gunata Z, Vallier M, Sapis J, Baumes R, Bayonove C (1994) Enzymatic synthesis of monoterpenyl $\beta$-D-glucosides by various $\beta$-glucosidases. Enzyme and microbial technology 16 (12):1055-1058

Guth H (1997) Quantitation and Sensory Studies of Character Impact Odorants of Different White Wine Varieties. Journal of Agricultural and Food Chemistry 45 (8):3027-3032. doi:10.1021/jf970280a

Hampel D, Robinson AL, Johnson A, Ebeler SE (2014) Direct hydrolysis and analysis of glycosidically bound aroma compounds in grapes and wines: comparison of hydrolysis conditions and sample preparation methods. Australian journal of grape and wine research 20 (3):361-377

Harbertson JF, Keller M (2011) Rootstock Effects on Deficit-Irrigated Winegrapes in a Dry Climate: Grape and Wine Composition. American Journal of Enology and Viticulture 63 (1):40-48. doi:10.5344/ajev.2011.11079

Hernandez-Orte P, Concejero B, Astrain J, Lacau B, Cacho J, Ferreira V (2015) Influence of viticulture practices on grape aroma precursors and their relation with wine aroma. Journal of the Science of Food and Agriculture 95 (4):688-701

Hjelmeland AK, Ebeler SE (2014) Glycosidically Bound Volatile Aroma Compounds in Grapes and Wine: A Review. American Journal of Enology and Viticulture 66 (1):1-11. doi:10.5344/ajev.2014.14104

Hjelmeland AK, Zweigenbaum J, Ebeler SE (2015) Profiling monoterpenol glycoconjugation in Vitis vinifera L. cv. Muscat of Alexandria using a novel putative compound database approach, high resolution mass spectrometry and collision induced dissociation fragmentation analysis. Anal Chim Acta 887:138147. doi:10.1016/j.aca.2015.06.026

Hogan S, Zhang L, Li J, Zoecklein B, Zhou K (2009) Antioxidant properties and bioactive components of Norton (Vitis aestivalis) and Cabernet Franc (Vitis vinifera) wine grapes. LWT-Food Science and Technology 42 (7):1269-1274

Holopainen JK, Blande JD (2012) Molecular plant volatile communication. In: Sensing in nature. Springer, pp 17-31 
Ilc T, Werck-Reichhart D, Navrot N (2016) Meta-analysis of the core aroma components of grape and wine aroma. Frontiers in Plant Science 7:1472

Jackson RS (2008) Wine science: principles and applications. Academic press,

James G, Witten D, Hastie T, Tibshirani R (2013) An introduction to statistical learning, vol 112. Springer,

Jogaiah S, Striegler KR, Bergmeier E, Harris J (2012) Influence of cluster exposure to Sun on fruit composition of 'Norton' grapes (vitis estivalis michx) in Missouri. International journal of fruit science 12 (4):410-426

Jogaiah S, Striegler KR, Bergmeier E, Harris J (2013) Influence of canopy management practices on canopy characteristics, yield, and fruit composition of 'Norton'grapes (Vitis aestivalis Michx). International journal of fruit science 13 (4):441-458

Jones T, Cullis BR, Clingeleffer PR, Rühl E (2009a) Effects of novel hybrid and traditional rootstocks on vigour and yield components of Shiraz grapevines. Australian Journal of Grape and Wine Research 15 (3):284-292

JONES TH, CULLIS BR, CLINGELEFFER PR, RÜHL EH (2009b) Effects of novel hybrid and traditional rootstocks on vigour and yield components of Shiraz grapevines. Australian Journal of Grape and Wine Research 15 (3):284-292. doi:10.1111/j.1755-0238.2009.00061.x

Kalua C, Boss PK (2010) Comparison of major volatile compounds from Riesling and Cabernet Sauvignon grapes (Vitis vinifera L.) from fruitset to harvest. Australian Journal of Grape and Wine Research 16 (2):337-348

Kalua CM, Boss PK (2009) Evolution of volatile compounds during the development of Cabernet Sauvignon grapes (Vitis vinifera L.). Journal of agricultural and food chemistry 57 (9):3818-3830

Katajamaa M, Miettinen J, Orešič M (2006) MZmine: toolbox for processing and visualization of mass spectrometry based molecular profile data. Bioinformatics 22 (5):634-636

Keller M, Mills LJ, Harbertson JF (2012) Rootstock effects on deficit-irrigated winegrapes in a dry climate: Vigor, yield formation, and fruit ripening. American Journal of Enology and Viticulture 63 (1):29-39

Kennedy J (2002) Understanding grape berry development. Practical winery \& vineyard $4: 1-5$

Kliewer W (1965) Changes in Concentration of Glucose, Fructose, and Total Soluble Solids in Flowers and Berries of Vitis Vlnifera. American Journal of Enology and Viticulture 16 (2):101-110

Kliewer WM, Howarth L, Omori M (1967) Concentrations of tartaric acid and malic acids and their salts in Vitis vinifera grapes. American Journal of Enology and Viticulture 18 (1):42-54

Kolor MG (1983) Identification of an important new flavor compound in Concord grape: ethyl-3-mercaptopropionate. Journal of Agricultural and Food Chemistry 31 (5):1125-1127

Kotseridis Y, Baumes RL, Skouroumounis GK (1999) Quantitative determination of free and hydrolytically liberated $\beta$-damascenone in red grapes and wines using a stable isotope dilution assay. Journal of Chromatography A 849 (1):245-254. doi:https://doi.org/10.1016/S0021-9673(99)00540-3 
Koundouras S, Hatzidimitriou E, Karamolegkou M, Dimopoulou E, Kallithraka S, Tsialtas JT, Zioziou E, Nikolaou N, Kotseridis Y (2009) Irrigation and rootstock effects on the phenolic concentration and aroma potential of Vitis vinifera L. cv. cabernet sauvignon grapes. J Agric Food Chem 57 (17):7805-7813. doi:10.1021/jf901063a

Koundouras S, Tsialtas IT, Zioziou E, Nikolaou N (2008) Rootstock effects on the adaptive strategies of grapevine (Vitis vinifera L. cv. Cabernet-Sauvignon) under contrasting water status: Leaf physiological and structural responses. Agriculture, Ecosystems \& Environment 128 (1):86-96. doi:https://doi.org/10.1016/j.agee.2008.05.006

Kwasniewski MT, Vanden Heuvel JE, Pan BS, Sacks GL (2010) Timing of cluster light environment manipulation during grape development affects $\mathrm{C} 13$ norisoprenoid and carotenoid concentrations in Riesling. Journal of agricultural and food chemistry 58 (11):6841-6849

Lee J-E, Hwang G-S, Van Den Berg F, Lee C-H, Hong Y-S (2009) Evidence of vintage effects on grape wines using $1 \mathrm{H}$ NMR-based metabolomic study. Analytica chimica acta 648 (1):71-76

Liland KH (2011) Multivariate methods in metabolomics - from pre-processing to dimension reduction and statistical analysis. TrAC Trends in Analytical Chemistry 30 (6):827-841. doi:https://doi.org/10.1016/j.trac.2011.02.007

Liu D, Xing R-R, Li Z, Yang D-M, Pan Q-H (2016) Evolution of volatile compounds, aroma attributes, and sensory perception in bottle-aged red wines and their correlation. European Food Research and Technology 242 (11):1937-1948. doi:10.1007/s00217-016-2693-1

Liu HF, Wu BH, Fan PG, Li SH, Li LS (2006) Sugar and acid concentrations in 98 grape cultivars analyzed by principal component analysis. Journal of the Science of Food and Agriculture 86 (10):1526-1536

Main G (2005) Growing and vinting Cynthiana. Norton grapes. Proceedings of the 24th Annual Horticulture Industries Show, Fort Smith, AR, USA,

Main G, Morris J, Striegler K (2002) Rootstock effects on Chardonel productivity, fruit, and wine composition. American Journal of Enology and Viticulture 53 (1):37-40

Main GL, Morris JR (2008) Impact of pruning methods on yield components and juice and wine composition of Cynthiana grapes. American journal of enology and viticulture 59 (2):179-187

Mansfield AK, Schirle-Keller J-P, Reineccius GA (2011) Identification of odor-impact compounds in red table wines produced from Frontenac grapes. American journal of enology and viticulture:ajev. 2011.10067

Mateo J, Jiménez M (2000) Monoterpenes in grape juice and wines. Journal of chromatography A 881 (1-2):557-567

Mato I, Suárez-Luque S, Huidobro JF (2005) A review of the analytical methods to determine organic acids in grape juices and wines. Food Research International 38 (10):1175-1188

Mendes-Pinto MM (2009) Carotenoid breakdown products the-norisoprenoids - in wine aroma. Archives of Biochemistry and Biophysics 483 (2):236-245. doi:https://doi.org/10.1016/j.abb.2009.01.008 
Migicovsky Z, Harris ZN, Klein LL, Li M, McDermaid A, Chitwood DH, Fennell A, Kovacs LG, Kwasniewski M, Londo JP, Ma Q, Miller AJ (2019) Rootstock effects on scion phenotypes in a 'Chambourcin' experimental vineyard. Horticulture Research 6 (1):64. doi:10.1038/s41438-019-0146-2

Mirás-Avalos JM, Intrigliolo DS (2017) Grape Composition under Abiotic Constrains: Water Stress and Salinity. Frontiers in Plant Science 8 (851). doi:10.3389/fpls.2017.00851

Moyer J, Mattick L (1976) Determination of methyl anthranilate in wines. American Journal of Enology and Viticulture 27 (3):134-135

Mudge K, Janick J, Scofield S, Goldschmidt EE (2009) 9 A History of Grafting. Horticultural reviews 35:437

Narduzzi L, Stanstrup J, Mattivi F (2015) Comparing Wild American Grapes with Vitis vinifera: A Metabolomics Study of Grape Composition. J Agric Food Chem 63 (30):6823-6834. doi:10.1021/acs.jafc.5b01999

Nelson CC, Kennedy JA, Zhang Y, Kurtural SK (2015) Applied Water and Rootstock Affect Productivity and Anthocyanin Composition of Zinfandel in Central California. American Journal of Enology and Viticulture 67 (1):18-28. doi:10.5344/ajev.2015.15043

Nelson R, Acree T, Lee C, Butts R (1977) Methyl anthranilate as an aroma constituent of American wine. Journal of Food Science 42 (1):57-59

Nisbet MA, Martinson TE, Mansfield AK (2014) Accumulation and Prediction of Yeast Assimilable Nitrogen in New York Winegrape Cultivars. American Journal of Enology and Viticulture 65 (3):325-332. doi:10.5344/ajev.2014.13130

Noble A, Cole V (2003) Flavor chemistry. In: Fermented Beverage Production. Springer, pp 393-412

Noble AC, Ebeler SE (2002) Use of multivariate statistics in understanding wine flavor. Food Reviews International 18 (1):1-20. doi:10.1081/fri-120003414

Olarte Mantilla SM, Collins C, Iland PG, Kidman CM, Ristic R, Boss PK, Jordans C, Bastian SEP (2018) Shiraz (<em>Vitis vinifera</em> L.) Berry and Wine Sensory Profiles and Composition Are Modulated by Rootstocks. American Journal of Enology and Viticulture 69 (1):32-44. doi:10.5344/ajev.2017.17017

Ollat N, Bordenave L, Tandonnet J-P, Boursiquot J-M, Marguerit E Grapevine rootstocks: origins and perspectives. In: I International Symposium on Grapevine Roots 1136, 2014. pp 11-22

Otto M (2016) Chemometrics: statistics and computer application in analytical chemistry. John Wiley \& Sons,

Ough C, Cook J, Lider L (1968) Rootstock-scion interactions concerning wine making. II. Wine compositional and sensory changes attributed to rootstock and fertilizer level differences. American Journal of Enology and Viticulture 19 (4):254-265

Parker M, Capone DL, Francis IL, Herderich MJ (2017) Aroma Precursors in Grapes and Wine: Flavor Release during Wine Production and Consumption. J Agric Food Chem. doi:10.1021/acs.jafc.6b05255

Pineau B, Barbe J-C, Van Leeuwen C, Dubourdieu D (2007) Which Impact for $\beta$ Damascenone on Red Wines Aroma? Journal of Agricultural and Food Chemistry 55 (10):4103-4108. doi:10.1021/jf070120r 
Plata C, Millan C, Mauricio J, Ortega J (2003) Formation of ethyl acetate and isoamyl acetate by various species of wine yeasts. Food Microbiology 20 (2):217-224

Polaskova P, Herszage J, Ebeler SE (2008) Wine flavor: chemistry in a glass. Chem Soc Rev 37 (11):2478-2489. doi:10.1039/b714455p

Poole C (2010) Chromatography: Concepts and Contrasts. Springer,

Qian MC, Fang Y, Shellie K (2009) Volatile Composition of Merlot Wine from Different Vine Water Status. Journal of Agricultural and Food Chemistry 57 (16):74597463. doi:10.1021/jf9009558

Rambla JL, Trapero-Mozos A, Diretto G, Rubio-Moraga A, Granell A, Gomez-Gomez L, Ahrazem O (2016) Gene-Metabolite Networks of Volatile Metabolism in Airen and Tempranillo Grape Cultivars Revealed a Distinct Mechanism of Aroma Bouquet Production. Front Plant Sci 7:1619. doi:10.3389/fpls.2016.01619

Rapp A, Mandery H (1986) Wine aroma. Experientia 42 (8):873-884

Reisch BI, Goodman RN, Martens M-H, Weeden NF (1993) The Relationship Between Norton and Cynthiana, Red. Wine Cultivars Derived from Vitis aestivalis. American journal of enology and viticulture 44 (4):441-444

Reisch BI, Owens CL, Cousins PS (2012) Grape. In: Fruit breeding. Springer, pp 225262

Reynolds AG, Fuleki T, Evans W (1982) Inheritance of methyl anthranilate and total volatile esters in Vitis spp. American Journal of Enology and Viticulture 33 (1):14-19

Reynolds AG, Lowrey WD, Tomek L, Hakimi J, De Savigny C (2007) Influence of irrigation on vine performance, fruit composition, and wine quality of Chardonnay in a cool, humid climate. American Journal of Enology and Viticulture 58 (2):217-228

Reynolds AG, Wardle DA (2001) Rootstocks impact vine performance and fruit composition of grapes in British Columbia. HortTechnology 11 (3):419-427

Rice S, Tursumbayeva M, Clark M, Greenlee D, Dharmadhikari M, Fennell A, Koziel JA (2019) Effects of harvest time on the aroma of white wines made from cold-hardy Brianna and Frontenac gris grapes using headspace solid-phase microextraction and gas chromatography-mass spectrometry-olfactometry. Foods 8 (1):29

Ristic R, Iland PG (2005) Relationships between seed and berry development of Vitis vinifera L. cv Shiraz: developmental changes in seed morphology and phenolic composition. Australian Journal of grape and wine research 11 (1):43-58

Robinson AL, Boss PK, Solomon PS, Trengove RD, Heymann H, Ebeler SE (2014) Origins of grape and wine aroma. Part 1. Chemical components and viticultural impacts. American Journal of Enology and Viticulture 65 (1):1-24

Robinson AL, Ebeler SE, Heymann H, Boss PK, Solomon PS, Trengove RD (2009) Interactions between wine volatile compounds and grape and wine matrix components influence aroma compound headspace partitioning. J Agric Food Chem 57 (21):10313-10322. doi:10.1021/jf902586n

Romero P, Botía P, del Amor FM, Gil-Muñoz R, Flores P, Navarro JM (2019) Interactive effects of the rootstock and the deficit irrigation technique on wine composition, nutraceutical potential, aromatic profile, and sensory attributes under semiarid and 
water limiting conditions. Agricultural Water Management 225:105733. doi:https://doi.org/10.1016/j.agwat.2019.105733

Roullier-Gall C, Witting M, Gougeon RD, Schmitt-Kopplin P (2014a) High precision mass measurements for wine metabolomics. Frontiers in Chemistry 2 (102). doi:10.3389/fchem.2014.00102

Roullier-Gall C, Witting M, Gougeon RD, Schmitt-Kopplin P (2014b) High precision mass measurements for wine metabolomics. Frontiers in chemistry 2:102

Ruffner H (1982a) Metabolism of tartaric and malic acids in Vitis V a review. A Vitis-,: $247 \mathrm{~V} 259$

Ruffner H (1982b) Metabolism of tartaric and malic acids in Vitis: A review-Part B. Vitis 21 (247-259):65

Ryona I, Sacks GL (2013) Behavior of glycosylated monoterpenes, C13-norisoprenoids, and benzenoids in Vitis vinifera cv. Riesling during ripening and following hedging. In: Carotenoid cleavage products. ACS Publications, pp 109-124

Sabbatini P, Howell GS (2013) Rootstock scion interaction and effects on vine vigor, phenology, and cold hardiness of interspecific hybrid grape cultivars (Vitis spp.). International journal of fruit science 13 (4):466-477

Sacks GL, Gates MJ, Ferry FX, Lavin EH, Kurtz AJ, Acree TE (2012) Sensory threshold of 1,1,6-trimethyl-1,2-dihydronaphthalene (TDN) and concentrations in young Riesling and non-Riesling wines. J Agric Food Chem 60 (12):2998-3004. doi:10.1021/jf205203b

Saito K, Matsuda F (2010) Metabolomics for functional genomics, systems biology, and biotechnology. Annu Rev Plant Biol 61:463-489. doi:10.1146/annurev.arplant.043008.092035

Sapkota S, Chen L-L, Schreiner K, Ge H, Hwang C-F (2015) A phenotypic study of Botrytis bunch rot resistance in Vitis aestivalis-derived 'Norton'grape. Tropical Plant Pathology 40 (4):279-282

Sapkota S, Chen L-L, Yang S, Hyma KE, Cadle-Davidson L, Hwang C-F (2019) Construction of a high-density linkage map and QTL detection of downy mildew resistance in Vitis aestivalis-derived 'Norton'. Theoretical and applied genetics 132 (1):137-147

Savoi S, Wong DCJ, Arapitsas P, Miculan M, Bucchetti B, Peterlunger E, Fait A, Mattivi F, Castellarin SD (2016) Transcriptome and metabolite profiling reveals that prolonged drought modulates the phenylpropanoid and terpenoid pathway in white grapes (Vitis vinifera L.). BMC Plant Biology 16 (1):67. doi:10.1186/s12870-016-0760-1

Schrimpe-Rutledge AC, Codreanu SG, Sherrod SD, McLean JA (2016) Untargeted Metabolomics Strategies - Challenges and Emerging Directions. Journal of The American Society for Mass Spectrometry 27 (12):1897-1905. doi:10.1007/s13361-016-1469-y

Schueuermann C, Khakimov B, Engelsen SB, Bremer P, Silcock P (2016) GC-MS Metabolite Profiling of Extreme Southern Pinot noir Wines: Effects of Vintage, Barrel Maturation, and Fermentation Dominate over Vineyard Site and Clone Selection. Journal of Agricultural and Food Chemistry 64 (11):2342-2351. doi:10.1021/acs.jafc.5b05861 
Schueuermann C, Steel CC, Blackman JW, Clark AC, Schwarz LJ, Moraga J, Collado IG, Schmidtke LM (2019) A GC-MS untargeted metabolomics approach for the classification of chemical differences in grape juices based on fungal pathogen. Food chemistry 270:375-384

Schwab W, Wüst M (2015) Understanding the Constitutive and Induced Biosynthesis of Mono- and Sesquiterpenes in Grapes (Vitis vinifera): A Key to Unlocking the Biochemical Secrets of Unique Grape Aroma Profiles. Journal of Agricultural and Food Chemistry 63 (49):10591-10603. doi:10.1021/acs.jafc.5b04398

Sefton MA, Skouroumounis GK, Elsey GM, Taylor DK (2011) Occurrence, Sensory Impact, Formation, and Fate of Damascenone in Grapes, Wines, and Other Foods and Beverages. Journal of Agricultural and Food Chemistry 59 (18):9717-9746. doi:10.1021/jf201450q

Serra I, Strever A, Myburgh P, Deloire A (2014) the interaction between rootstocks and cultivars (V itis vinifera L.) to enhance drought tolerance in grapevine. Australian Journal of Grape and Wine Research 20 (1):1-14

Shure KB, Acree TE (1994) Changes in the odor-active compounds in Vitis labruscana cv. Concord during growth and development. Journal of agricultural and food chemistry 42 (2):350-353

Skinkis PA, Bordelon BP, Wood KV (2008) Comparison of monoterpene constituents in Traminette, Gewürztraminer, and Riesling winegrapes. American journal of enology and viticulture 59 (4):440-445

Slegers A, Angers P, Ouellet E, Truchon T, Pedneault K (2015) Volatile Compounds from Grape Skin, Juice and Wine from Five Interspecific Hybrid Grape Cultivars Grown in Quebec (Canada) for Wine Production. Molecules 20 (6):10980-11016. doi:10.3390/molecules200610980

Slegers A, Angers P, Pedneault K (2017) Volatile Compounds from Must and Wines from Five White Grape Varieties. Journal of Food Chemistry and Nanotechnology 03 (01). doi:10.17756/jfcn.2017-031

Stockert CM, Bisson LF, Adams DO, Smart DR (2013) Nitrogen status and fermentation dynamics for Merlot on two rootstocks. American Journal of Enology and Viticulture 64 (2):195-202

Striegler R, Howell G (2015) The influence of rootstock on the cold-hardiness of Seyval grapevines 1 . Primary and secondary effects on growth, canopy development, yield, fruit quality and cold hardiness. VITIS-Journal of Grapevine Research 30 (1): 1

Striegler R, Howell G, Flore J (1993) Influence of rootstock on the response of Seyval grapevines to flooding stress. American journal of enology and viticulture 44 (3):313-319

Šuklje K, Carlin S, Stanstrup J, Antalick G, Blackman JW, Meeks C, Deloire A, Schmidtke LM, Vrhovsek U (2019) Unravelling wine volatile evolution during Shiraz grape ripening by untargeted HS-SPME-GC $\times$ GC-TOFMS. Food chemistry 277:753-765

Sun Q, Gates MJ, Lavin EH, Acree TE, Sacks GL (2011a) Comparison of odor-active compounds in grapes and wines from Vitis vinifera and non-foxy American grape species. Journal of agricultural and food chemistry 59 (19):10657-10664 
Sun Q, Gates MJ, Lavin EH, Acree TE, Sacks GL (2011b) Comparison of odor-active compounds in grapes and wines from vitis vinifera and non-foxy American grape species. J Agric Food Chem 59 (19):10657-10664. doi:10.1021/jf2026204

Sun Q, Sacks G, Lerch S, Heuvel JEV (2011c) Impact of shoot thinning and harvest date on yield components, fruit composition, and wine quality of Marechal Foch.

American journal of enology and viticulture 62 (1):32-41

Sweetman C, Deluc LG, Cramer GR, Ford CM, Soole KL (2009) Regulation of malate metabolism in grape berry and other developing fruits. Phytochemistry 70 (1112):1329-1344

Tautenhahn R, Patti GJ, Rinehart D, Siuzdak G (2012) XCMS Online: a web-based platform to process untargeted metabolomic data. Analytical chemistry 84 (11):5035-5039

Team RC (2019) R: A Language and Environment for Statistical Computing (Version 3.5. 2, R Foundation for Statistical Computing, Vienna, Austria, 2018). There is no corresponding record for this reference[Google Scholar]

Teh SL, Rostandy B, Awale M, Luby JJ, Fennell A, Hegeman AD (2019) Genetic analysis of stilbenoid profiles in grapevine stems reveals a major mQTL hotspot on chromosome 18 associated with disease-resistance motifs. Horticulture research $6(1): 1-11$

This P, Lacombe T, Thomas MR (2006) Historical origins and genetic diversity of wine grapes. Trends Genet 22 (9):511-519. doi:10.1016/j.tig.2006.07.008

Thomas AL, Harris JL, Bergmeier EA, Striegler RK (2017) Establishment Technique and Rootstock Impact 'Chambourcin'Grapevine Morphology and Production in Missouri. HortTechnology 27 (2):248-256

Toffali K, Zamboni A, Anesi A, Stocchero M, Pezzotti M, Levi M, Guzzo F (2011) Novel aspects of grape berry ripening and post-harvest withering revealed by untargeted LC-ESI-MS metabolomics analysis. Metabolomics 7 (3):424-436. doi:10.1007/s11306-010-0259-y

Tramontini S, Vitali M, Centioni L, Schubert A, Lovisolo C (2013) Rootstock control of scion response to water stress in grapevine. Environmental and experimental botany 93:20-26

Van Wyk C, Augustyn O, De Wet P, Joubert W (1979) Isoamyl acetate - a key fermentation volatile of wines of Vitis vinifera cv Pinotage. American Journal of Enology and Viticulture 30 (3):167-173

Villamor RR, Ross CF (2013) Wine matrix compounds affect perception of wine aromas. Annual review of food science and technology 4:1-20

Vivian Z, Thibaut V, Agnès D, Sandrine B, Fabrice L, Carole K, Katia G, Jorge ES, Olivier V, Jean-Laurent S (2018) The impact of plant water status on the gas exchange, berry composition and wine quality of Chasselas grapes in Switzerland. OENO One 52 (4). doi:10.20870/oeno-one.2018.52.4.2181

Vogt J, Schiller D, Ulrich D, Schwab W, Dunemann F (2013) Identification of lipoxygenase (LOX) genes putatively involved in fruit flavour formation in apple (Malus $\times$ domestica). Tree genetics \& genomes 9 (6):1493-1511 
Vrhovsek U, Lotti C, Masuero D, Carlin S, Weingart G, Mattivi F (2014) Quantitative metabolic profiling of grape, apple and raspberry volatile compounds (VOCs) using a GC/MS/MS method. Journal of Chromatography B 966:132-139

Wang Y, Chen W-K, Gao X-T, He L, Yang X-H, He F, Duan C-Q, Wang J (2019) Rootstock-Mediated Effects on Cabernet Sauvignon Performance: Vine Growth, Berry Ripening, Flavonoids, and Aromatic Profiles. International journal of molecular sciences 20 (2):401

Want E, Masson P (2011) Processing and analysis of GC/LC-MS-based metabolomics data. In: Metabolic profiling. Springer, pp 277-298

Warschefsky EJ, Klein LL, Frank MH, Chitwood DH, Londo JP, von Wettberg EJ, Miller AJ (2016) Rootstocks: diversity, domestication, and impacts on shoot phenotypes. Trends in plant science 21 (5):418-437

Winterhalter P, Rouseff R (2001) Carotenoid-Derived Aroma Compounds: An Introduction. In: Carotenoid-Derived Aroma Compounds, vol 802. ACS Symposium Series, vol 802. American Chemical Society, pp 1-17. doi:doi:10.1021/bk-2002-0802.ch001

10.1021/bk-2002-0802.ch001

Wooldridge J, Louw P, Conradie W (2010) Effects of rootstock on grapevine performance, petiole and must composition, and overall wine score of Vitis vinifera cv. Chardonnay and Pinot Noir. South African Journal of Enology and Viticulture 31 (1):45-48

Xia J, Wishart DS (2016) Using MetaboAnalyst 3.0 for Comprehensive Metabolomics Data Analysis. Current Protocols in Bioinformatics 55 (1):14.10.11-14.10.91. doi:10.1002/cpbi.11

Xu C-H, Chen G-S, Xiong Z-H, Fan Y-X, Wang X-C, Liu Y (2016) Applications of solid-phase microextraction in food analysis. TrAC Trends in Analytical Chemistry 80:12-29. doi:https://doi.org/10.1016/j.trac.2016.02.022

Young PR, Eyeghe-Bickong HA, du Plessis K, Alexandersson E, Jacobson DA, Coetzee Z, Deloire A, Vivier MA (2016) Grapevine plasticity in response to an altered microclimate: Sauvignon Blanc modulates specific metabolites in response to increased berry exposure. Plant physiology 170 (3):1235-1254

Yuan F, Qian MC (2016) Development of C13-norisoprenoids, carotenoids and other volatile compounds in Vitis vinifera L. Cv. Pinot noir grapes. Food chemistry 192:633-641

Zhang E, Chai F, Zhang H, Li S, Liang Z, Fan P (2017) Effects of sunlight exclusion on the profiles of monoterpene biosynthesis and accumulation in grape exocarp and mesocarp. Food chemistry 237:379-389

Zhang L, Marguerit E, Rossdeutsch L, Ollat N, Gambetta GA (2016) The influence of grapevine rootstocks on scion growth and drought resistance. Theoretical and Experimental Plant Physiology 28 (2):143-157. doi:10.1007/s40626-016-0070-x 
6. Figures:

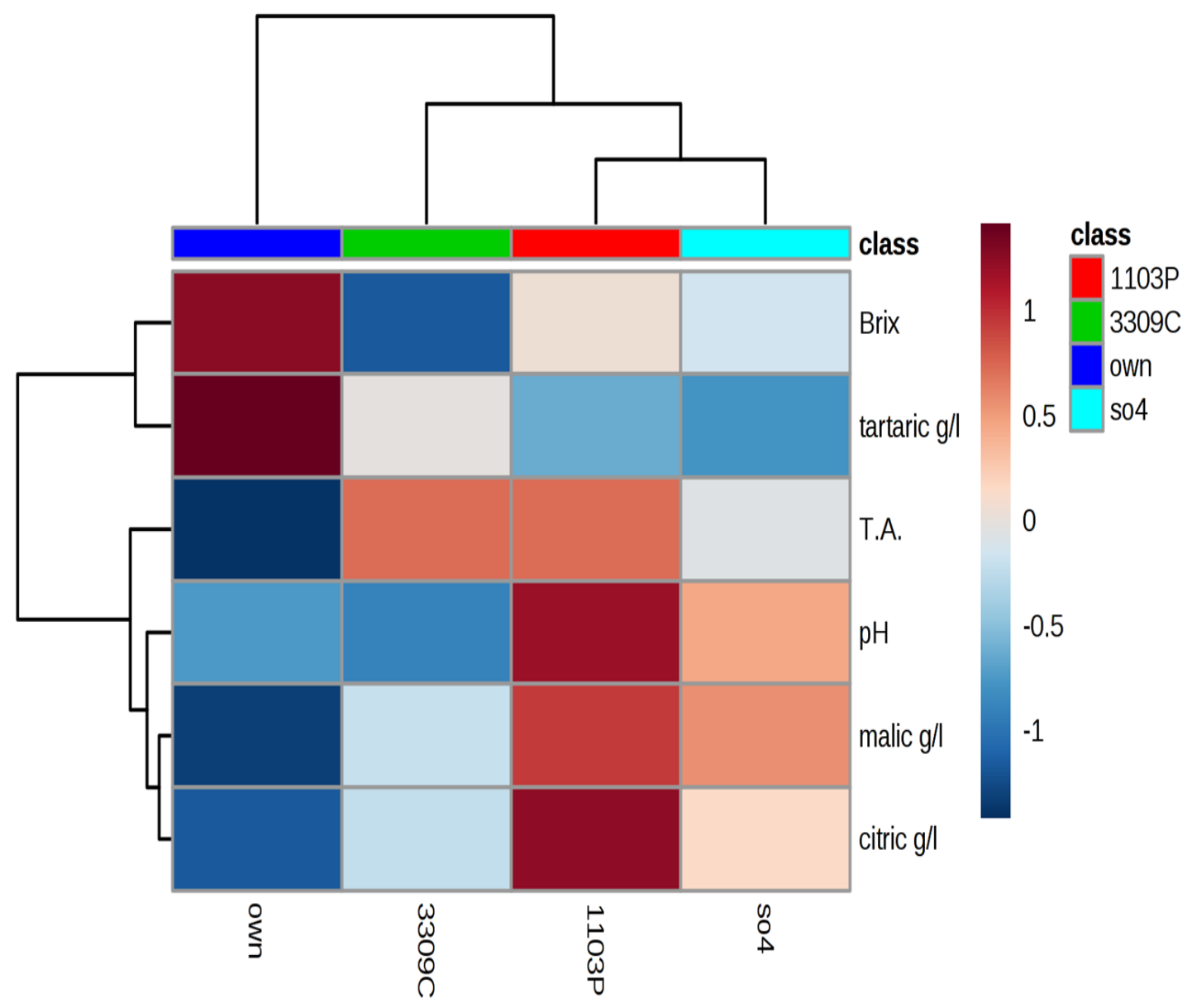

Figure 5.1: Heatmap of basic berry chemistry traits differences between different rootstocks grafted to Chambourcin and own-rooted Chambourcin. 

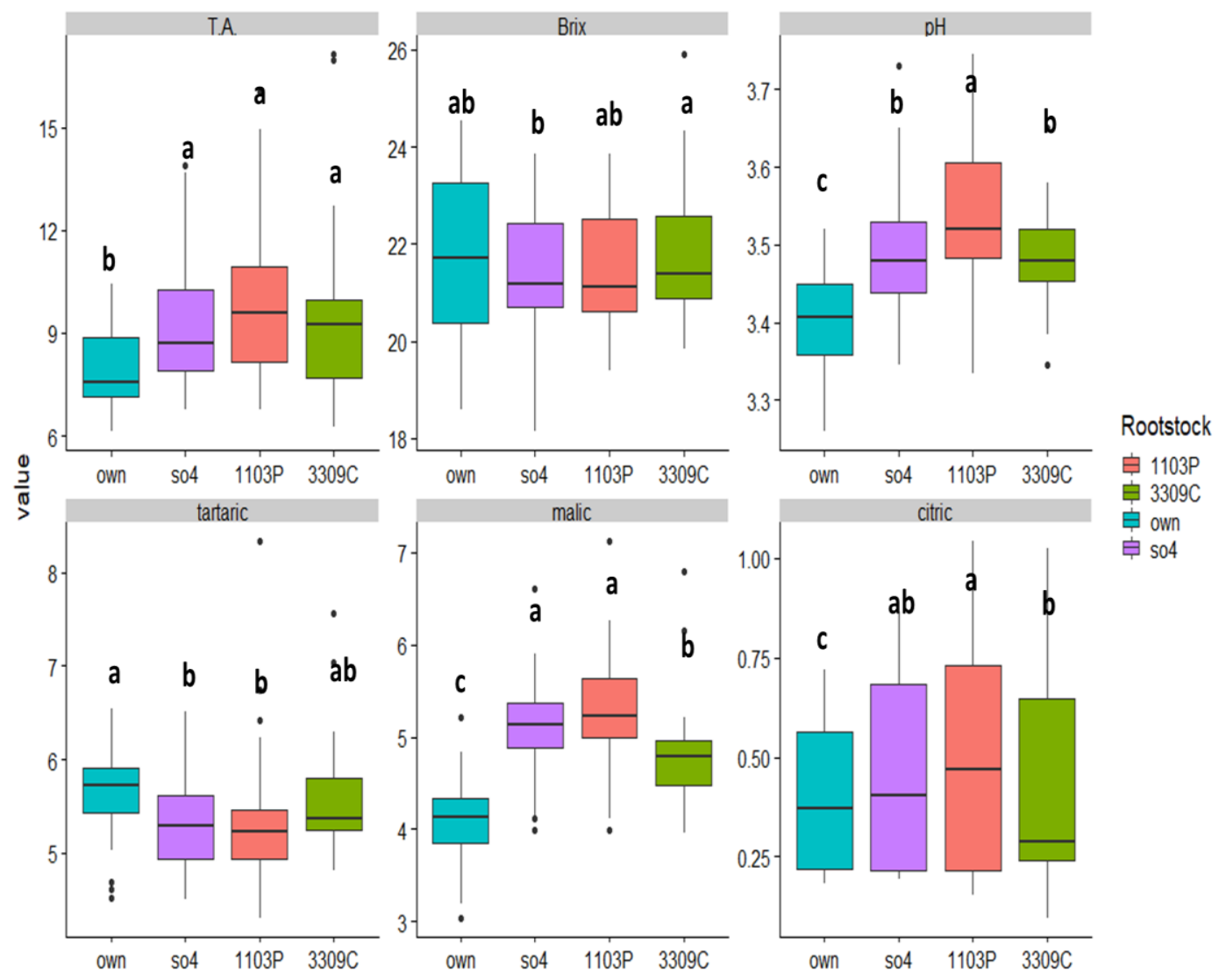

Figure 5.2: Boxplots of berry chemistry traits (Titratable acidity (TA), Total soluble solids (brix), $\mathrm{pH}$, Tartaric acid, malic acid and citric acid) differences due to rootstocks (1103P, 3309Cand SO4) and own-rooted on Chambourcin scion. The values or concentrations are average of two years (2017 and 2018). The different letters in the boxplots represent significant difference (Duncan Multiple test). 

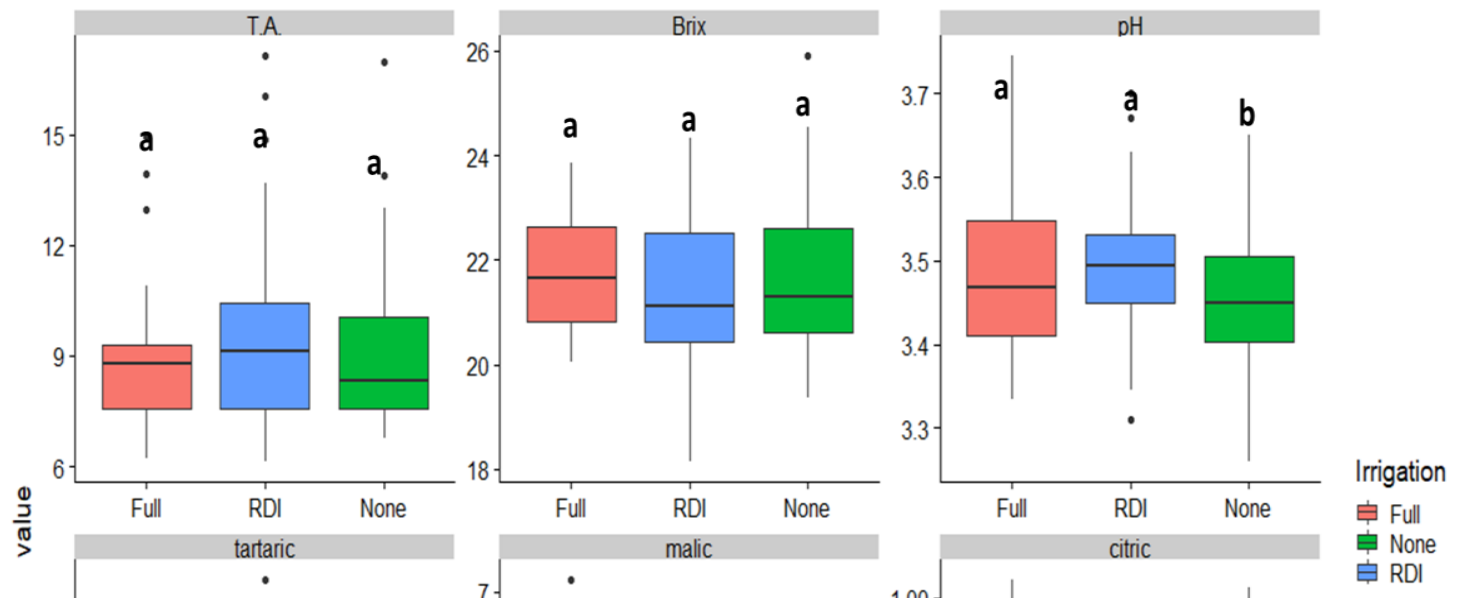

Figure 5.3: Boxplots demonstrating impact of irrigation treatments (Full, RDI and None) on each berry chemistry traits (Titratable acidity (TA), Total soluble solids (brix), $\mathrm{pH}$, Tartaric acid, malic acid and citric acid). The different letters signify significant differences (Duncan Multiple test). The values are the average of two years. 

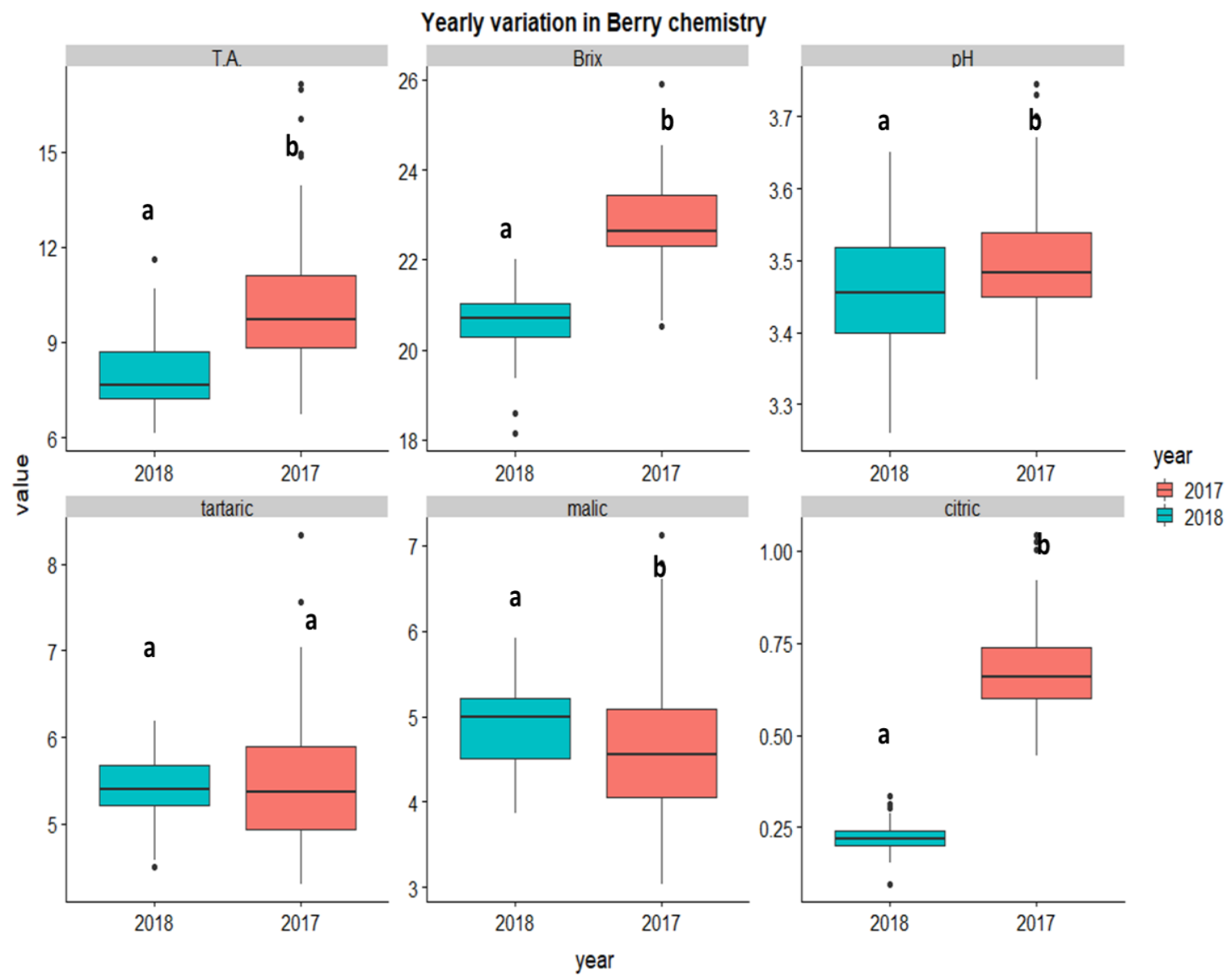

Figure 5.4: Boxplots demonstrating yearly differences in concentration of the individual berry chemistry traits (Titratable acidity (TA), Total soluble solids (Brix), $\mathrm{pH}$ ), and organic acids (Tartaric acid, malic acid, and citric acid). The different letters signify significant differences between years (Duncan multiple tests). 


\section{Rootstock effect on Berry Chemistry}
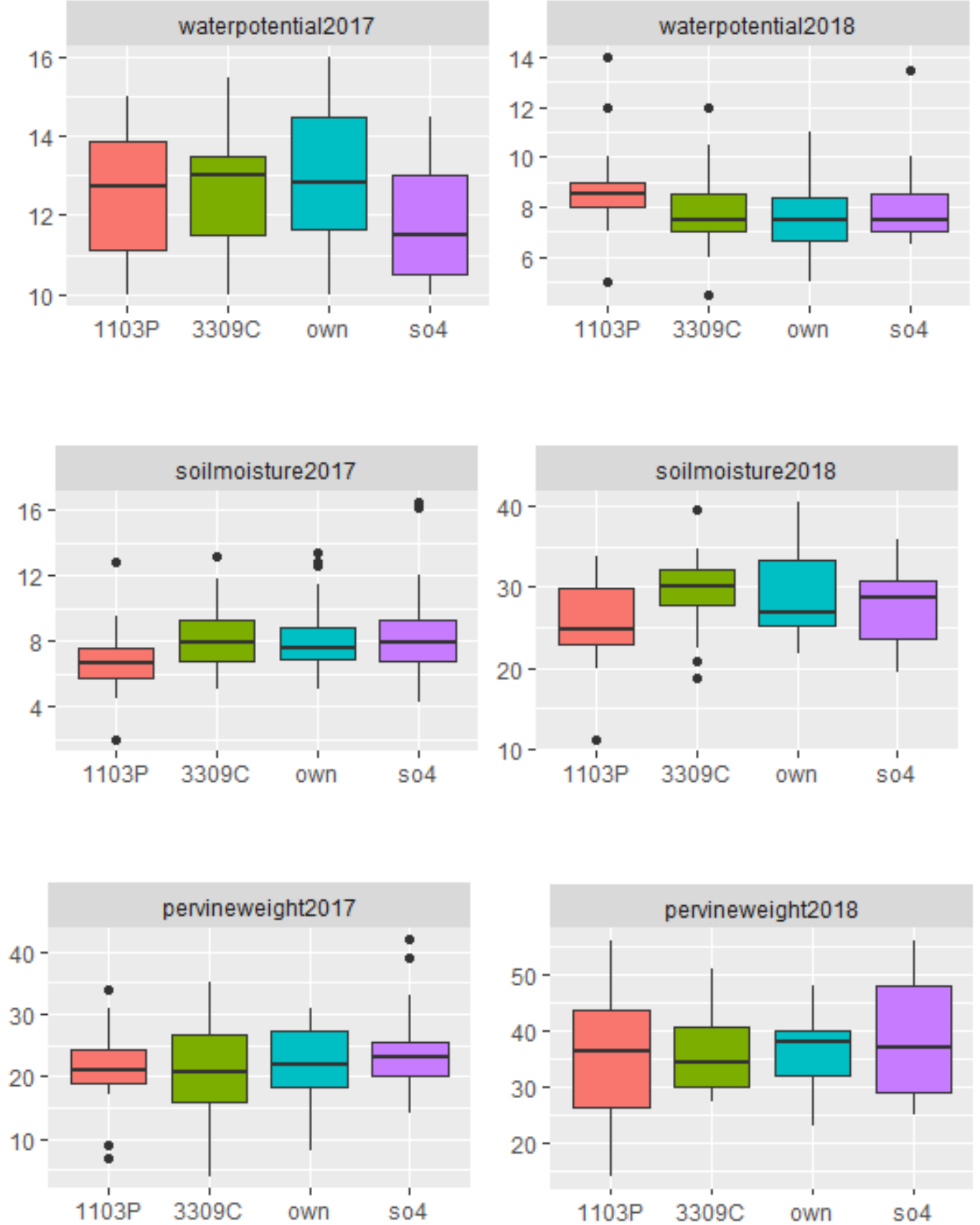

Figure 5.5: Boxplots of midday leaf water potential (bars) and soil moisture content, berry weight per vine differences due to rootstocks (1103P, 3309Cand SO4), and ownrooted on Chambourcin scion in 2017 and 2018. 


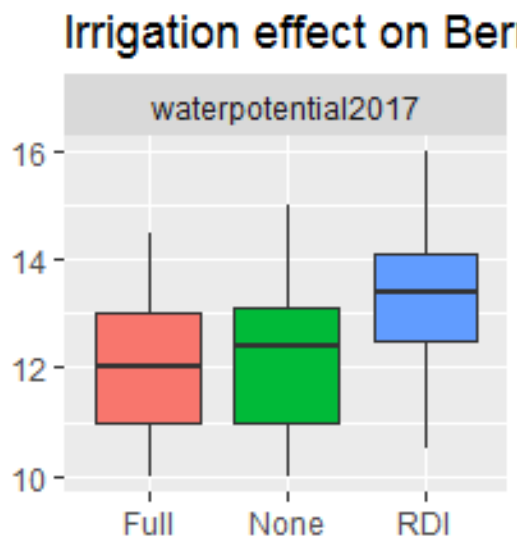

\section{Chemistry}
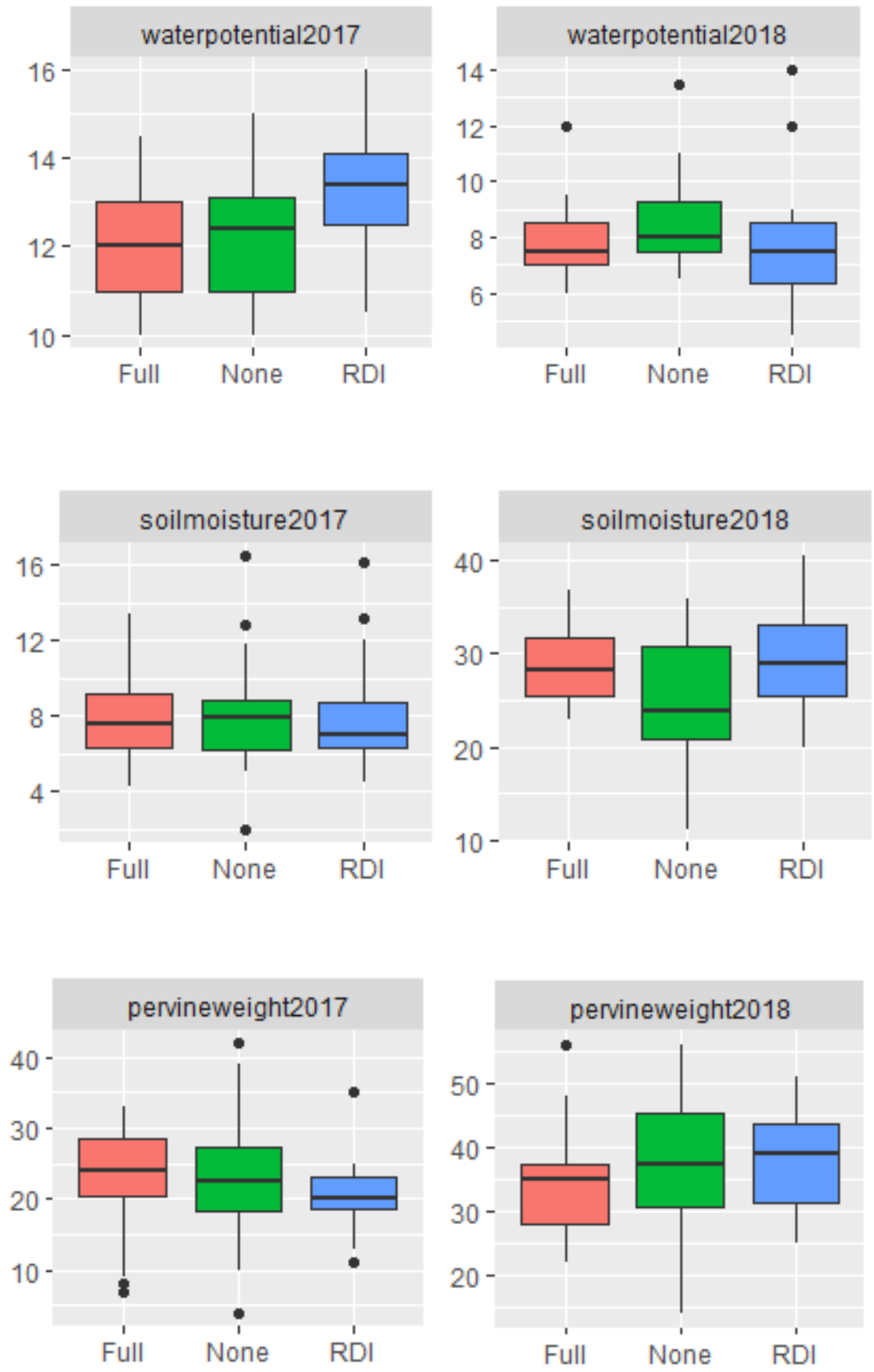

Figure 5.6: Boxplots of pressure chamber measurements(bars) and soil moisture content, berry weight per vine differences due to irrigation regimes(Full, RDI, and None) in 2017 and 2018. 


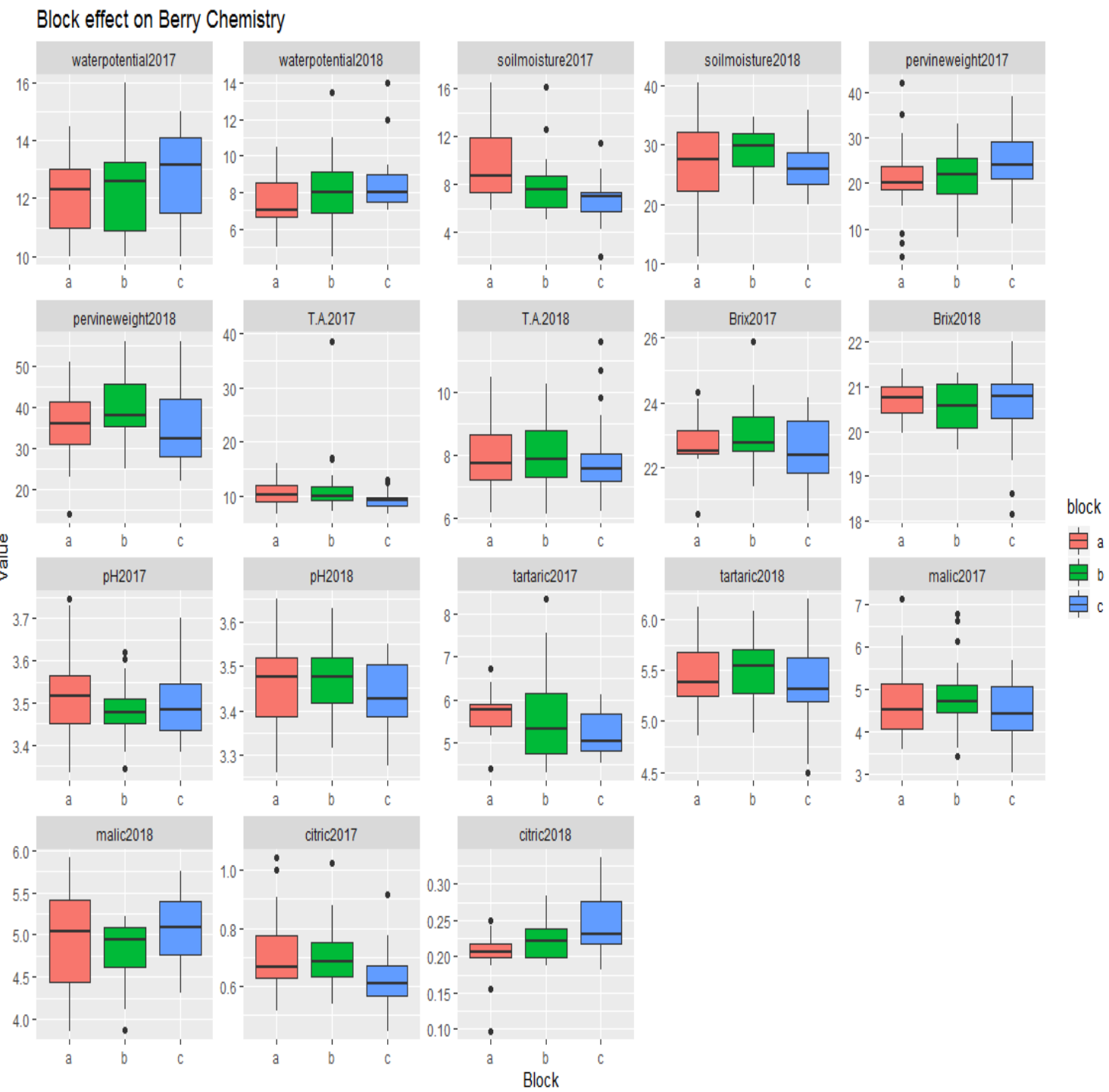

Figure 5.7: Boxplots demonstrating differences in midday leaf water potential (bars) and soil moisture content, berry weight per vine and concentration of the individual berry chemistry traits (Titratable acidity (TA), Total soluble solids (Brix), pH) and organic acids (Tartaric acid, malic acid, and citric acid) by blocks (position in the vineyard) for both 2017 and 2018. 


\section{Table:}

Table 5.1: Mean concentrations of basic berry chemistry (Titratable acidity (TA), Total soluble solids (Brix), pH), organic acids (Tartaric acid, malic acid, and citric acid), and water potential of Chambourcin berries differed according to rootstocks and irrigation regimes.

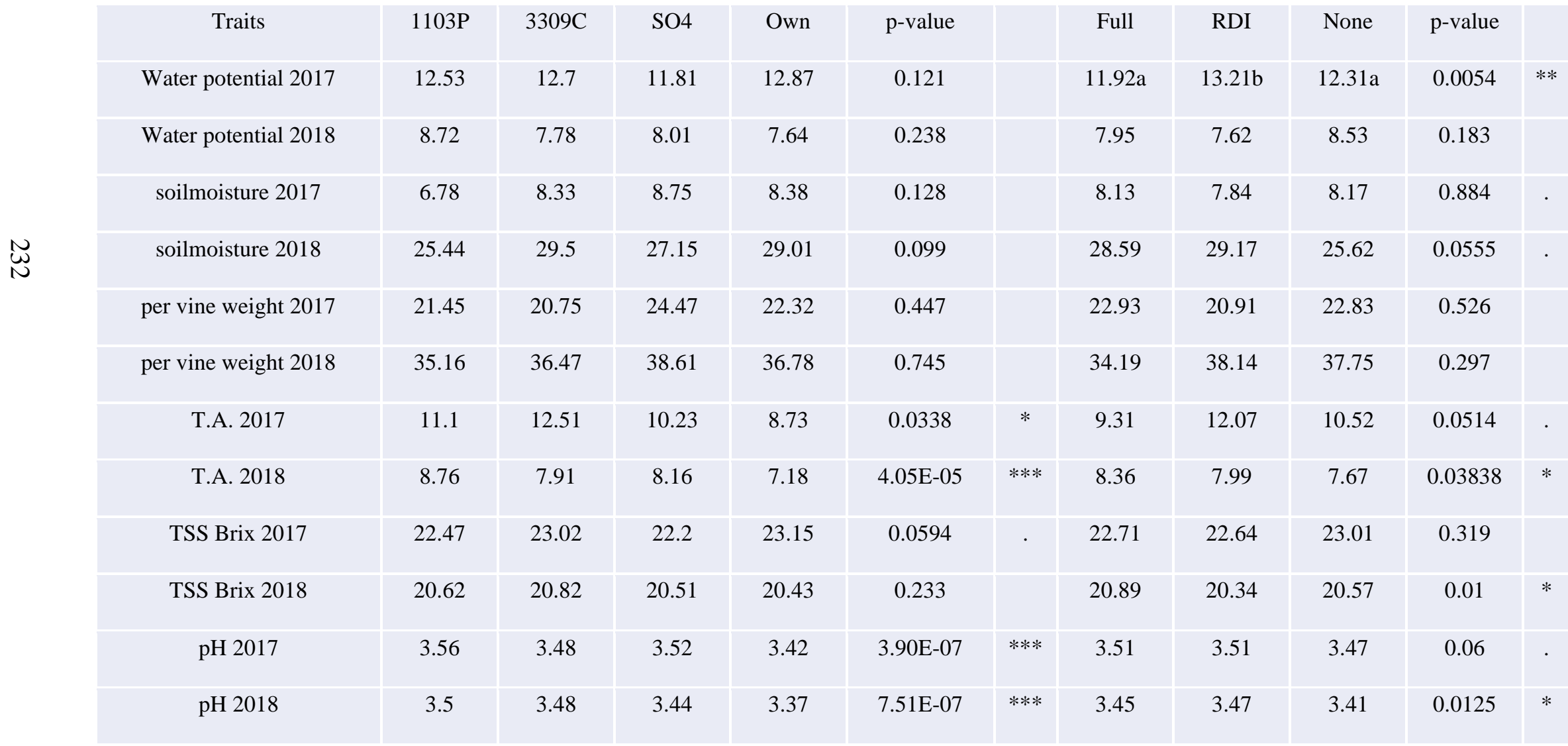




\begin{tabular}{|c|c|c|c|c|c|c|c|c|c|c|c|}
\hline tartaric acid 2017 & 5.42 & 5.66 & 5.31 & 5.58 & $5.10 \mathrm{E}-01$ & & 5.42 & 5.64 & 5.43 & 0.522 & \\
\hline tartaric acid 2018 & 5.23 & 5.51 & 5.29 & 5.7 & $3.38 \mathrm{E}-05$ & $* * *$ & 5.34 & 5.62 & 5.34 & 0.00167 & $* *$ \\
\hline malic acid 2017 & 5.2 & 4.69 & 5.02 & 3.86 & 4.04E-07 & $* * *$ & 4.53 & 4.85 & 4.68 & 0.295 & \\
\hline malic acid 2018 & 5.36 & 4.9 & 5.13 & 4.31 & $4.51 \mathrm{E}-14$ & $* * *$ & 4.8 & 5 & 4.96 & 0.0963 & . \\
\hline citric acid 2017 & 0.76 & 0.68 & 0.69 & 0.58 & $6.76 \mathrm{E}-05$ & $* * *$ & 0.66 & 0.69 & 0.67 & 0.493 & \\
\hline citric acid 2018 & 0.22 & 0.22 & 0.21 & 0.23 & $6.14 \mathrm{E}-01$ & & 0.2 & 0.22 & 0.23 & 0.0348 & * \\
\hline
\end{tabular}




\section{Chapter 6: Conclusions and future works:}

Berry free and total volatiles as well as wine volatiles are very important for the quality of the wine and are impacted by many factors including the genetics of the scion and rootstocks, various management activities in the vineyard, winemaking decisions and environmental factors such as rainfall, sunshine, climate and soil conditions. Through this

study, we found that genetics of the scion is the major determinant of the variation in berry and wine volatiles. Chapter 1 of this dissertation demonstrated that the Norton and Cabernet Sauvignon berries and wines are different from each other for free, total, and wine volatiles. Both of these cultivars are genetically different as Cabernet Sauvignon is a $V$. vinifera cultivar and Norton is an interspecific cultivar derived from V. aestivalis. Norton was found to contain higher concentrations of defense-related volatiles including methyl salicylate and eugenol in comparison to Cabernet Sauvignon which might be responsible for the disease resistance nature of Norton. When developing cultivars that are consumer-preferred, care should be taken not to take away too much disease resistance of the plants. Using the key volatiles different between Norton and Cabernet Sauvignon to phenotype the F1 population, we found that these metabolic traits are segregating and under the influence of polygenes and thus can be located using genetic analysis. This also sheds light on the importance of understanding the phenotypic differences in parents and their inheritance before jumping directly into crossing two parents to produce a breeding population. Phenotyping the parents beforehand gives the idea of what key differences we should be looking into in the breeding population. 
We also found that genetics of the rootstocks also play a significant role in berry and wine volatiles, although the differences were much small compared to that of the scion. In Chapters 3 and 4, we demonstrated that different rootstocks grafted to the same scion Chambourcin differed from the own-rooted Chambourcin for the berry and wine volatiles with some volatiles increased due to grafting and some decreased in grafted vines. The effects were subtle but significant. The wine was found to demonstrate a greater and significant impact of rootstocks compared to the berries. The fact that rootstocks had only subtle effects on berry volatiles but a major impact on the relative concentration of wine volatiles raises the possibility that the principal manner by which rootstocks modulate wine volatiles may not be the concentration of berry metabolites. It is possible that other factors, such as grape-derived enzymes that modify or release volatile compounds during fermentation, are the ones through which rootstocks primarily influence wine quality.

Water availability to the plants as manipulated through different irrigation systems was also found to have a significant but subtle impact on the berry and wine volatiles. Regulated deficit irrigation was found to be completely from both full and none irrigation. In years with higher water stress, RDI treated vines were found to accumulate higher concentrations of important volatiles, for example, $\beta$-Damascenone was found to be relatively higher in concentrations in RDI vines. There is also significant rootstock by irrigation interaction impact in wine volatiles. This demonstrated that through management practices such as irrigation, the berry and wine volatiles can be manipulated to prepare wines that are consumer preferred. 
The metabolomics-based approach used in this study is a better approach to study a complex system such as berries and wine. Also, because of its comprehensive and holistic approach, this approach proved beneficial in understanding understudied cultivars such as Norton and Chambourcin, without missing analytes that are of interest. While most of the metabolomics studies stop at identifying the compounds, we went forward and were able to confirm and quantify the compounds that are causing differences between cultivars or differences due to rootstocks and irrigation.

To understand how rootstock genetics impact the differences in berry and wine volatiles, the metabolomics data can be integrated with the genomics data such as berry RNAseq data (collected at the same time from the same vines), which might be able to explain the how the genes contributed to the differences in berry and wine volatiles. Similarly, looking at the metabolomics data through the lens of physiology data such as photosynthesis data or basic berry chemistry data or water potential data might be able to explain some of the changes that we see between rootstocks and own-rooted berries as well changes induced due to deficit irrigation. Also, to better explain how the berry volatiles and wine volatiles are related to each other, we could study the correlations between them to understand the predictability of the volatiles that will help to predict how the wines will look like based on the berry volatiles. Additionally, the metabolomics data can be integrated with the hyperspectral data (remote sensing data) to understand if the changes in volatiles can be captured by remote sensing data that will help to reduce the time spent in the field sampling. 


\section{VITA}

Born on December $5^{\text {th, }} 1988$ in a middle-class agricultural family in Kathmandu valley (Patan, Lalitpur) to Late. Herakaji Awale and Mrs. Mohan Maya Awale, Mani Awale developed an interest in agriculture from a very young age. With helping her parents and relatives to plant rice in summer and harvest in fall, she was always fascinated with the different varieties of rice that are grown. With the developing interests to learn more about agriculture, she joined the Institute of Agriculture and Animal Sciences, Tribhuvan University against her parent's wish to join the business school. Mani received her Bachelor of Science in Agriculture with a major in Plant Breeding and Genetics from Tribhuvan University, Nepal in 2011. After completion of her undergraduate, she joined Nepal Agriculture Research Council (NARC) as a volunteer research assistant and worked in the wheat varietal trial screening the varieties resistance to wheat rust. After that, in 2013, she joined the Department of Plant Sciences at South Dakota State University in Dr. Anne Fennell's lab. Her MS thesis was on the Phenotyping F1 population and identifying the QTL for freezing tolerance in grapes.

It was during one of the field visits in the local vineyard and winery that Mani developed a deep interest in the field of winemaking and quality. Having come from a traditional family where rice wine and alcoholic beverages are part of the culture, she had grown up observing and helping her mom and grandma prepare the traditional liquor at home. This somewhere had left the interest in the field of making beverages and she wanted to learn more about how wines are made from grapes and how the quality of wine can be improved. After completion of her MS degree in 2016, she joined the Division of 
Plant Sciences at the University of Missouri in Dr. Misha Kwasniewski's lab, where she worked in improving the hybrid grapes important to Missouri using the metabolomicsbased approach. She will receive her Ph.D. in Plant, Insect, and Microbial Science specializing in Viticulture and Enology in July 2020. 